National Water-Quality Assessment Program

\title{
Method for Estimating Annual Atrazine Use for Counties in the Conterminous United States, 1992-2007
}

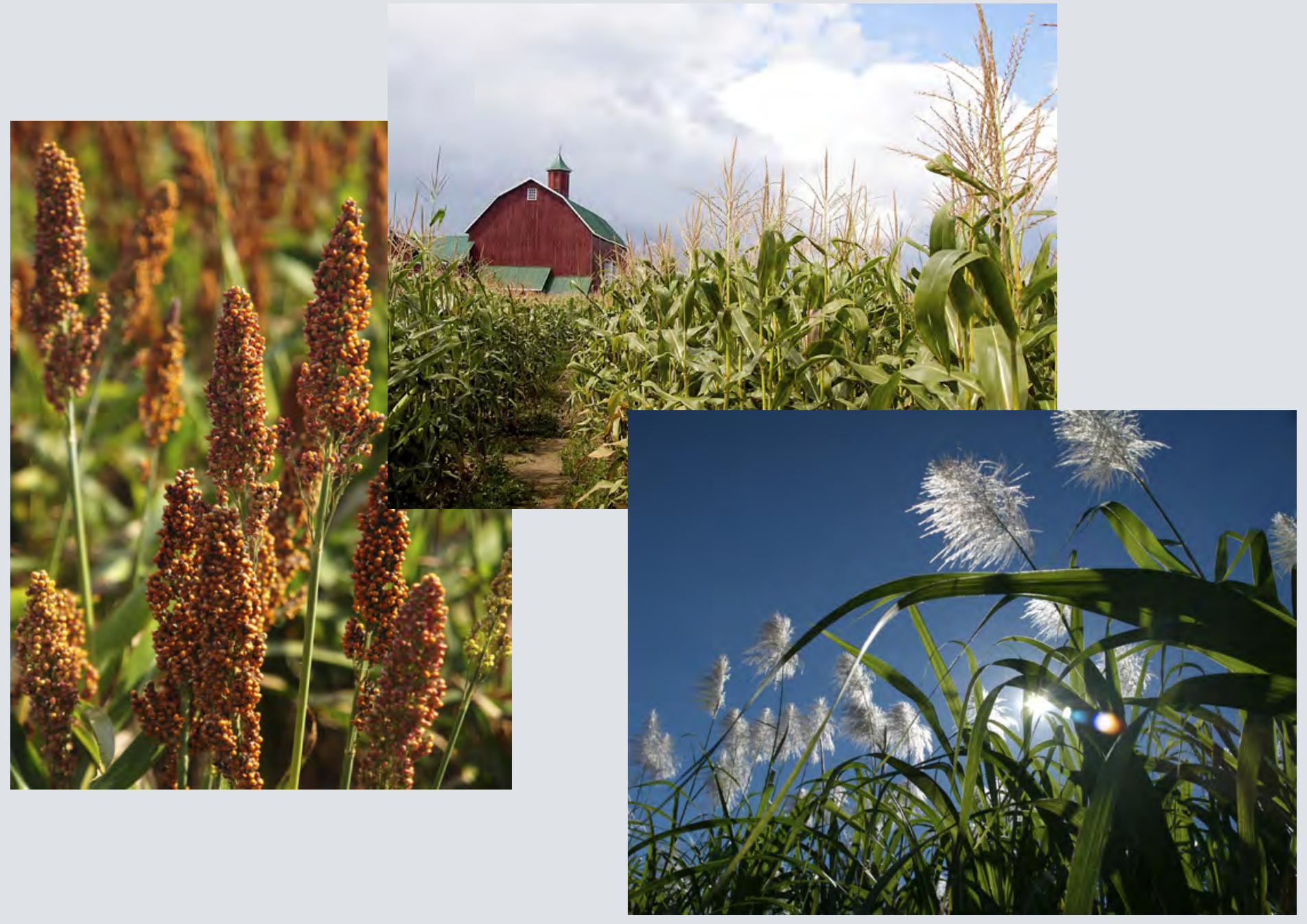

Scientific Investigations Report 2010-5034 
Left: Photograph showing heads of grain sorghum (milo). Middle: Photograph showing a red barn in a corn field.

Right: Photograph showing flowering sugar cane plants.

(Stock images purchased from istockphotos.com/) 


\section{Method for Estimating Annual Atrazine Use for Counties in the Conterminous United States, 1992-2007}

By Gail P. Thelin and Wesley W. Stone

National Water-Quality Assessment Program

Scientific Investigations Report 2010-5034 


\title{
U.S. Department of the Interior \\ KEN SALAZAR, Secretary
}

\section{U.S. Geological Survey \\ Marcia K. McNutt, Director}

\section{U.S. Geological Survey, Reston, Virginia: 2010}

\author{
For more information on the USGS — the Federal source for science about the Earth, its natural and living resources, \\ natural hazards, and the environment, visit http://www.usgs.gov or call 1-888-ASK-USGS \\ For an overview of USGS information products, including maps, imagery, and publications, \\ visit http://www.usgs.gov/pubprod \\ To order this and other USGS information products, visit http://store.usgs.gov
}

Any use of trade, product, or firm names is for descriptive purposes only and does not imply endorsement by the U.S. Government.

Although this report is in the public domain, permission must be secured from the individual copyright owners to reproduce any copyrighted materials contained within this report.

Suggested citation:

Thelin, G.P., and Stone, W.W., 2010, Method for estimating annual atrazine use for counties in the conterminous United States, 1992-2007: U.S. Geological Survey Scientific Investigations Report 2010-5034, 129 p. 


\section{Foreword}

The U.S. Geological Survey (USGS) is committed to providing the Nation with reliable scientific information that helps to enhance and protect the overall quality of life and that facilitates effective management of water, biological, energy, and mineral resources (http://www.usgs.gov/). Information on the Nation's water resources is critical to ensuring long-term availability of water that is safe for drinking and recreation and is suitable for industry, irrigation, and fish and wildlife. Population growth and increasing demands for water make the availability of that water, now measured in terms of quantity and quality, even more essential to the long-term sustainability of our communities and ecosystems.

The USGS implemented the National Water-Quality Assessment (NAWQA) Program in 1991 to support national, regional, state, and local information needs and decisions related to water-quality management and policy (http://water.usgs.gov/nawqa). The NAWQA Program is designed to answer: What is the quality of our Nation's streams and groundwater? How are conditions changing over time? How do natural features and human activities affect the quality of streams and groundwater, and where are those effects most pronounced? By combining information on water chemistry, physical characteristics, stream habitat, and aquatic life, the NAWQA Program aims to provide science-based insights for current and emerging water issues and priorities. From 1991-2001, the NAWQA Program completed interdisciplinary assessments and established a baseline understanding of water-quality conditions in 51 of the Nation's river basins and aquifers, referred to as Study Units (http://water.usgs.gov/nawqa/studyu.html).

National and regional assessments are ongoing in the second decade (2001-2012) of the NAWQA Program as 42 of the 51 Study Units are selectively reassessed. These assessments extend the findings in the Study Units by determining status and trends at sites that have been consistently monitored for more than a decade, and filling critical gaps in characterizing the quality of surface water and groundwater. For example, increased emphasis has been placed on assessing the quality of source water and finished water associated with many of the Nation's largest community water systems. During the second decade, NAWQA is addressing five national priority topics that build an understanding of how natural features and human activities affect water quality, and establish links between sources of contaminants, the transport of those contaminants through the hydrologic system, and the potential effects of contaminants on humans and aquatic ecosystems. Included are studies of the fate of agricultural chemicals, effects of urbanization on stream ecosystems, bioaccumulation of mercury in stream ecosystems, effects of nutrient enrichment on aquatic ecosystems, and transport of contaminants to public-supply wells. In addition, national syntheses of information on pesticides, volatile organic compounds (VOCs), nutrients, trace elements, and aquatic ecology are continuing.

The USGS aims to disseminate credible, timely, and relevant science information to address practical and effective water-resource management and strategies that protect and restore water quality. We hope this NAWQA publication will provide you with insights and information to meet your needs, and will foster increased citizen awareness and involvement in the protection and restoration of our Nation's waters.

The USGS recognizes that a national assessment by a single program cannot address all water-resource issues of interest. External coordination at all levels is critical for cost-effective management, regulation, and conservation of our Nation's water resources. The NAWQA Program, therefore, depends on advice and information from other agencies—-Federal, state, regional, interstate, tribal, and local—as well as nongovernmental organizations, industry, academia, and other stakeholder groups. Your assistance and suggestions are greatly appreciated. 
This page intentionally left blank. 


\section{Contents}

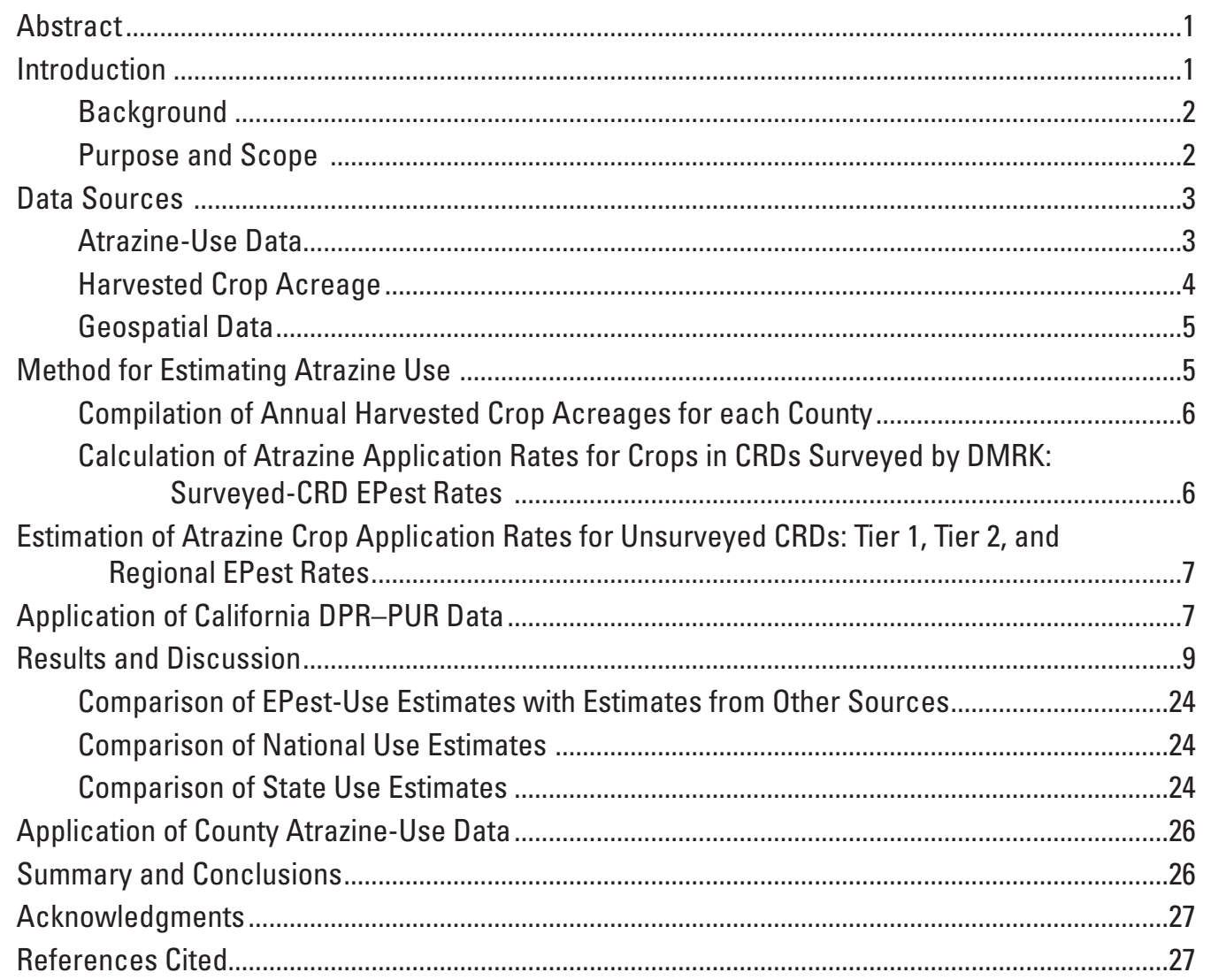




\section{Figures}

Figure 1. Map showing Crop Reporting Districts (CRD), counties, and U.S. Department of Agriculture (USDA) Farm Production Regions

Figure 2. Map showing example of a Primary Crop Reporting District (CRD) and surrounding Tier 1 and Tier 2 CRDs

Figure 3. Diagram showing summary of the decision process followed to estimate EPest rates and EPest use for unsurveyed Crop Reporting Districts (CRD)

Figure 4. Graph showing national use of atrazine on major crops and other crops, 1992-2007

Figure 5. Graphs showing dtribution of atrazine use by estimation method on all crops, on corn, sorghum, and sugar cane, and on minor crops, 1992-2007 .....

Figure 6. Maps showing distribution of atrazine use and the proportion estimated using an unsurveyed Crop Reporting District (CRD) rate, 1992-2007

Figure 7. Maps showing distribution of atrazine use on corn and estimates derived from an unsurveyed Crop Reporting District (CRD) rate,1992-2007

Figure 8. Graph showing percentage of atrazine use and acreage estimates derived from interpolated crop acreage, 1992-2007

Figure 9. Graphs showing comparison of Census of Agriculture and National Agriculture Statistics Service (NASS) estimates of corn acreage in a county for 1992, 1997, 2002, and 2007

Figure 10. Graph showing national atrazine-use estimates for agricultural purposes, 1992-2007

Figure 11. Graphs showing comparison of EPest and National Pesticide Use Database (NPUD) and National Agriculture Statistics Service Chemical Use (NASS-CU) estimates of state atrazine use on corn....

\section{Tables}

Table 1. Crop Reporting Districts (CRD) of the conterminous United States and associated counties

Table 2. Relation of Doane Marketing Research-Kynetec (DMRK) crop categories (not including California) to Census of Agriculture and National Agriculture Statistics Service crop categories used in the atrazine-use estimation process

Table 3. Mapping of California Department of Pesticide Regulation (DPR) crops to EPest crops and associated 1992 and 2001 National Land Cover Dataset (NLCD) crop categories reported in the county atrazine-use database.... 


\title{
Abbreviations and Acronyms
}

\author{
CRD Crop Reporting District \\ DMRK Doane Marketing Research-Kynetec \\ DPR-PUR Department of Pesticide Regulation-Pesticide Use Reporting (California) \\ FIPS Federal Information Processing Standards \\ FSA Farm Services Administration \\ EPest use pesticide use estimates derived from surveyed and EPest rates \\ EPest rate crop application rate derived from surveyed-CRD, Tier 1, Tier 2 or regional rates \\ GIS Geographic Information System \\ NASS National Agriculture Statistics Service \\ NASS-CU National Agriculture Statistics Service Chemical Use \\ NAWQA National Water Quality Assessment Program \\ NCFAP National Center for Food and Agricultural Policy \\ NPUD National Pesticide Use Database \\ TRS Township, Range, Section \\ USDA U.S. Department of Agriculture \\ USEPA U.S. Environmental Protection Agency \\ USGS U.S. Geological Survey
}


This page intentionally left blank. 


\title{
Method for Estimating Annual Atrazine Use for Counties in the Conterminous United States, 1992-2007
}

\author{
By Gail P. Thelin and Wesley W. Stone
}

\section{Abstract}

A method was developed to estimate annual atrazine use during 1992 to 2007 on sixteen crops and four agricultural land uses. For each year, atrazine use was estimated for all counties in the conterminous United States (except California) by combining (1) proprietary data from the Doane Marketing Research-Kynetec (DMRK) AgroTrak database on the mass of atrazine applied to agricultural crops, (2) county harvested crop acreage, by county, from the 1992, 1997, 2002, and 2007 Censuses of Agriculture, and (3) annual harvested crop acreage from National Agriculture Statistics Service (NASS) for non-Census years. DMRK estimates of pesticide use on individual crops were derived from surveys of major field crops and selected specialty crops in multicounty areas referred to as Crop Reporting Districts (CRD). The CRDlevel atrazine-use estimates were disaggregated to obtain county-level application rates by dividing the mass (pounds) of pesticides applied to a crop by the acreage of that crop in the CRD to yield a rate per harvested acre. When atrazineuse estimates were not available for a CRD, crop, or year, an estimated rate was developed following a hierarchy of decision rules that checked first for the availability of a crop application rate from surveyed atrazine application rate(s) for adjacent CRDs for a specific year, and second, the rates from surveyed CRDs within for U.S. Department of Agriculture Farm Production Regions for a specific year or multiple years. The estimation method applied linear interpolation to estimate crop acreage for years when harvested acres for a crop and county were not reported in either the Census of Agriculture or the NASS database, but were reported by these data sources for other years for that crop and county. Data for atrazine use for the counties in California was obtained from farmers' reports of pesticide use collected and published by the California Department of Pesticide Regulation-Pesticide Use Reporting (DPR-PUR) because these data are more complete than DMRK survey data. National and state annual atrazineuse totals derived by this method were compared with other published pesticide-use estimates and were highly correlated. The method developed is designed to be applicable to other pesticides for which there are similar data; however, for some pesticides that are applied to specialty crops, fewer surveys are usually available to estimate application rates and there are a greater number of years with unreported crop acreage, potentially resulting in greater uncertainty in use estimates.

\section{Introduction}

An important component of crop management is the application of pesticides to control weeds, insect infestations, plant diseases, and other pests. Pesticides have beneficial effects by improving the quality and yield of crops produced, but also may be transported to surface and groundwater where they may affect water quality adversely. Pesticide concentrations in streams vary widely across the United States and are influenced by many factors, such as the amount and timing of pesticide applications and the natural features of the area where they are applied, including soils, climate, and hydrology. In 1991, the U.S. Geological Survey (USGS) initiated the National Water Quality Assessment (NAWQA) Program to assess the quality of the Nation's surface-water and groundwater resources, including a national assessment of the effects of pesticides on water quality.

Nationally consistent current and historic information on the amount and geographic distribution of pesticide use is essential for designing NAWQA studies, interpreting waterquality data, assessing trends in pesticide use, and developing water-quality models. Readily available public data on pesticide use are incomplete and have coarse geographic and temporal coverage. Information on pesticide use is needed that has sufficient spatial and temporal resolution, as well as complete spatial coverage, in order to support a range of local, regional, and national studies. Methods are needed that enable estimation of pesticide use for areas and time periods that have not been surveyed. 


\section{Background}

The design of NAWQA national and regional studies is based on an environmental framework, which includes information on soils, hydrology, climate, land use and a variety of human-related attributes, including pesticide use. This framework is used to characterize the environmental setting of study areas and, in relation to pesticides, provides a context in which to explain their occurrence in the hydrologic environment and evaluate the processes that transport pesticides applied on the land surface to water. To date, pesticide-use information included in the NAWQA environmental framework and most interpretive studies has been limited primarily to agricultural uses. Information on agricultural use was developed by combining data on county harvested crop acres with state-level estimates of pesticidecrop application rates that were published in a series of three national pesticide-use databases compiled by the National Center for Food and Agricultural Policy (NCFAP) (Gianessi and Anderson, 1995; Gianessi and Marcelli, 2000) and most recently updated by CropLife Foundation (Gianessi and Reginar, 2006). Each of the three versions of the National Pesticide Use Database (NPUD) was centered on a year of the Census of Agriculture (1992, 1997, and 2002).

The NPUD databases contain state-level pesticide-use information for over 200 pesticides used on 87 agricultural crops, and include state totals of the amounts of pesticides applied (in pounds) and percentage of crop acres treated in each state. These data were compiled from many sources, including the U.S. Department of Agriculture (USDA) Agricultural Chemical Use Database (http://www. pestmanagement.info/nass/) for row crops, vegetables, and fruits and nut crops; crop profiles developed by crop specialists and agricultural extension agents; and annual data on pesticide use collected by the California Department of Pesticide Regulation-Pesticide Use Reporting, (DPR-PUR). NPUD estimates of the pounds of pesticides applied and percentage of acres treated were developed from typical application rates reported by the sources noted above. Although each NPUD database is centered on a specific Census year, the pesticide application rates and the state-level estimates are a combination of rates and estimates taken from a 4 to 5 year period. The data are not intended to represent actual pesticide use for a specific year, but instead represent broad use patterns for a multi-year period. For example, state estimates for a specific pesticide and crop in the NPUD 1997 database may include application rates that are based on information from 1997 for some states and 1996 or 1998 for other states. In addition, for some states and crops, the state-level pesticide-use estimates may be imputed from the application rate for a neighboring state(s). The NPUD 1992, 1997, and 2002 databases are publicly available and are comprehensive sources of state-level pesticide-use information for the conterminous United States (http://cipm.ncsu.edu/ croplife/).
The last NPUD database was published in 2002, and because of the temporal and geographic limitations of NPUD estimates, an alternative source of pesticide-use information was sought that could yield improved spatial resolution on an annual basis. Such data are not available from public sources. Doane Marketing Research-Kynetec (DMRK) is an agricultural marketing research firm that for the past 20 years has conducted annual crop surveys of over twenty-thousand United States farm operations. DMRK markets farm-related data through its proprietary electronic database, AgroTrak. AgroTrak data is used by industry to conduct market research and by private industry and the U.S. Environmental Protection Agency (USEPA) for such purposes as modeling applications and risk assessment. AgroTrak is the only source of annual pesticide-use data that is available for most states and for a large number of active ingredients. Like NPUD, however, these data are limited to agricultural pesticide uses only.

\section{Purpose and Scope}

This report describes a new method for developing annual county-level estimates of atrazine use on agricultural crops for all areas of the conterminous United States that had reported crop acreage and that likely (based on reported crops) had applications of atrazine during 1992-2007. The method described for atrazine is a prototype for additional applications to other pesticides. The method uses AgroTrak survey data on pesticide use and Census of Agriculture and NASS data on harvested crop acreages for counties in all conterminous states except California. California DPRPUR data were used for California counties. To ensure that atrazine use was accounted for in all geographic areas of the conterminous United States, the method derived atrazine crop application rates for CRDs not surveyed by DMRK from adjacent Crop Reporting Districts (CRD) that had been surveyed. CRDs consist of multiple adjacent counties in each state, which were grouped by DMRK to represent similar geography, climate, and cropping practices. Atrazine was selected for method development and demonstration because it is a major agricultural herbicide that has been used in all 48 contiguous states. From 1992 to 2007, atrazine use accounted for almost 10 percent of the total mass of all pesticides used in agriculture. County-level estimates produced by the method were used to produce national and state atrazine-use totals for comparison with other published atrazine-use data. An example application of these data is described that profiles atrazine-use intensity for 1992 through 1999 in selected watersheds. 


\section{Data Sources}

The method incorporates pesticide-use information and data on harvested crop acreage for each year for counties in the conterminous United States for 1992 through 2007. Pesticide use information came from two sources: (1) AgroTrak annual atrazine-use estimates by CRD for the conterminous United States, except California and, (2) annual reported atrazine-use by Township, Range and Section (TRS) from the DPR-PUR for California. Annual county harvested crop acreages from the Censuses of Agriculture and NASS surveys were used in conjunction with the AgroTrak atrazineuse estimates to calculate estimates of pesticide use by crop and allocate pesticide use estimates to individual counties. Harvested rather than planted acreage was used to estimate atrazine use because the Census of Agriculture reports harvested crop acreage for the crops reported by DMRK, but not planted acres. Although, NASS reports planted and harvested crop acreage, fewer crops are reported and data are not reported for all counties. Finally, GIS coverages of CRD-county boundaries and TRS-county boundaries were used to (1) associate and disaggregate the AgroTrak CRD data to counties, and (2) link and aggregate the DPR-PUR TRS atrazine data to counties. The GIS coverage of CRDs was also used to produce a table that contained the spatial relationships of CRDs. Each of these data sets is described below.

\section{Atrazine-Use Data}

The proprietary DMRK database, AgroTrak, includes pesticide-use estimates for 447 active ingredients collected as part of annual surveys of more than 80 crops, including major field crops, some fruit and specialty crops, and other agricultural land use such as pasture and rangeland. These annual crop surveys are based on a statistical sample of commercial farm operations distributed throughout the geographic area surveyed, which is a CRD or group of CRDs (fig. 1). A survey was designed for each crop according to the geographic region and size of farm operation. DMRK uses data from the Census of Agriculture on the number and size of farm operations in each state to determine the sampling rate in a state and a CRD. Farm operations included in the survey are selected from lists of agricultural producers compiled by the USDA, agricultural associations, agricultural publications, previous survey participants, and other sources. The DMRK survey design allocates a higher proportion of the sample (farms to be surveyed) to larger farm operations in order to characterize a greater percentage of crop acres and increase
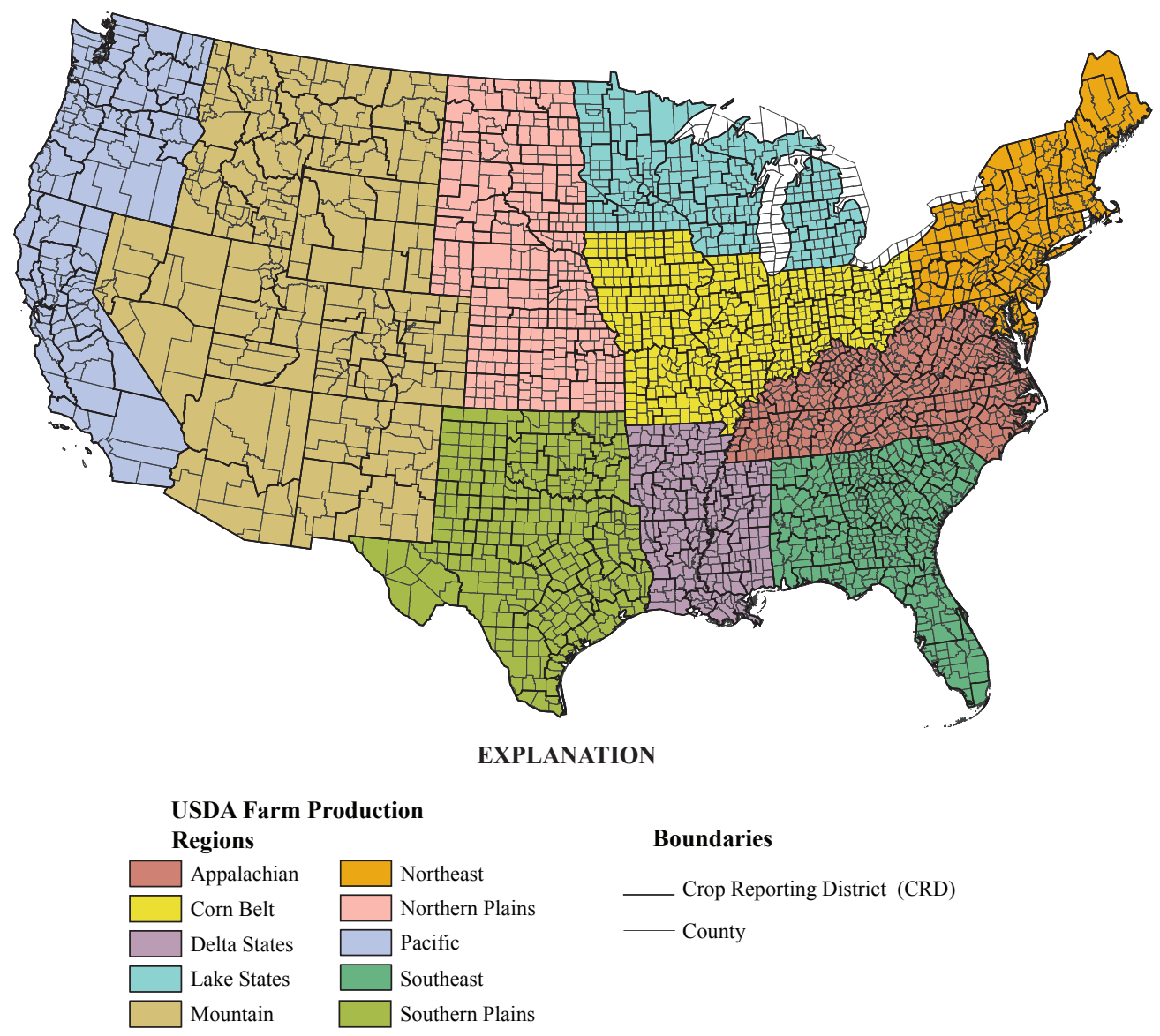

Figure 1. Crop Reporting Districts (CRD), counties, and U.S. Department of Agriculture (USDA) Farm Production Regions. 
accuracy. To adjust and correct for possible bias in survey responses, a modified regression weighting method was used which calculates a weighting factor (on the basis of Census of Agriculture data on number and size of farm operations) for each response. This process ensures that the sum of survey responses when expanded to the survey area (the $\mathrm{CRD}$ and the state) agree with known crop acreage estimates from the Census of Agriculture or NASS (John Thompson, DMRKynetec, written commun., January 2009).

Prior to entering the survey data collected from farm operations into AgroTrak, the survey responses are reviewed by DMRK and verified for accuracy as part of a data quality check. Data checks include comparing each survey respondent's report of crop application rates, timing, and method of application with the USEPA-approved pesticide application rates that are published in the USEPA's Pesticide Product Label System (http://www.epa.gov/pesticides/ pestlabels/). Additional checks verify that the amount applied is accurately reported in the survey response by multiplying the reported planted acres by the reported crop application rate to verify if the calculated and reported mass are equal. Data are also compared with patterns of historical use, and when necessary, DMRK contacts the survey respondent to review and verify the data submitted. In some cases, outside industry consultants also review rates and the geographic patterns of use reported.

Pesticide-use data collected by the California Department of Pesticide Regulation (DPR-PUR) (http://www.cdpr.ca.gov/ docs/pur/purmain.htm) was used instead of AgroTrak data to estimate pesticide use for California because the data are generally considered the most complete and accurate source of pesticide-use information for all areas of the State. DPR instituted a pesticide data collection program in 1990, which requires that all commercial pesticide applications be reported to the agricultural commissioner in the county where the pesticide was applied. For each pesticide applied to a crop, pesticide applicators are required to report the date and time of application, the number of planted and treated acres for the crop, the method and rate of application, and the location of use referenced to township, range and section (TRS). This information is entered into a county-level database which is uploaded and merged into the state electronic DPR-PUR database.

Pesticide-use information from AgroTrak and DPR-PUR were used to estimate county-level atrazine use by crop from 1992 to 2007. Data elements from AgroTrak reports used in this study included surveyed CRD reports on: (1) total pounds of atrazine applied on planted crop acres and (2) the number of planted crop acres. DPR-PUR data included information on the pounds of atrazine applied to individual crops by date and TRS.

\section{Harvested Crop Acreage}

The method developed to estimate county atrazine use requires county crop acreage estimates for the crop categories included in the AgroTrak database in order to (1) calculate atrazine-application rates for each harvested crop and CRD surveyed, and (2) estimate county atrazine use for all crops in all counties in the conterminous United States where atrazine was likely used by applying the CRD crop application rate to county-level harvested crop acreage. Harvested crop acreage per county was obtained from two independent USDA sources: Census of Agriculture data from 1992, 1997, 2002, and 2007 (http://www.agcensus.usda.gov/), and NASS for non-Census years (http://www.nass.usda.gov/Data and Statistics/Quick_Stats/) (U.S. Department of Agriculture, National Agricultural Statistics Service, variously dated).

The Census of Agriculture collects information every five years on land use and ownership, operator characteristics, production practices, income, and other farm-related topics for every farm, ranch and agricultural business that produces or sells $\$ 1,000$ or more of agricultural products. The Census is the only source of uniform and comprehensive farm-related data for every county in the United States and is considered a reliable and consistent source of harvested crop acreage data for row crops, specialty crops, fruit and nut crops, fallow land, and pasture.

Although NASS reports acreage for many planted crops, NASS annual crop surveys are not a complete enumeration of farm operations as in the Census of Agriculture, but rather data are collected from a subset of farm operations selected on the basis of a statistically designed survey of major crop production states. NASS does not report crop acreage for all counties in the conterminous United States. NASS county data include estimates of planted and harvested acres for major field crops, including major grains, oilseeds, edible beans, sugar crops, cotton, and tobacco. Other crops for which data are collected include hay, potatoes, minor oil seeds, and fruit crops; the data are not collected directly through NASS surveys, but through cooperative programs with state departments of agriculture and land-grant universities. Although the list of crops that is included in annual NASS surveys is not as extensive as the Census of Agriculture, NASS surveys provide harvested crop acreage data for many of the crops needed to estimate atrazine use for non-Census years. Although for atrazine, as well as many other pesticides, planted crop acreage would probably be the preferred estimate of actual treated acreage, but the sole availability of harvested acreage data in the Census and the high correlation between planted and harvested acreage made it preferable to use harvested acreage as the basis of estimates. 


\section{Geospatial Data}

A geographic information system (GIS) was used to develop a geospatial coverage of United States CRDs and associated counties so that pesticide-use data in AgroTrak reported by CRD could be spatially allocated to CRDs and counties. There are 304 CRDs in the conterminous United States (fig. 1). CRDs are similar to NASS Agricultural Statistic Districts (ASD). The geographic attributes used to define the CRDs include soil type, terrain, and elevation and climatic factors, such as mean temperature, annual precipitation, and length of growing season. All of these factors influence the types of crops grown and crop management practices. Most states are divided into nine CRDs; however, some states, such as Massachusetts and New Hampshire, contain only one CRD, while Texas contains 15 CRDs.

A geospatial vector dataset, hereinafter referred to as GIS coverage, of the 304 CRD boundaries was developed in a two step process. First, the CRD codes and related county Federal Information Processing Standard (FIPS) codes given in table 1 (all tables shown in back of report) were joined to a 1:100,000-scale GIS coverage of 2001-defined county boundaries that contained county FIPS codes (JoAnn Gronberg, written commun., June 2008). Next, a GIS function was used to dissolve or eliminate the county boundaries that were inside the CRD boundary. The GIS CRD coverage was used to generate a table that contained the spatial relationship between each of the 304 CRDs (primary) and its surrounding CRDs. Any CRD can be considered a primary or a Tier 1 or a Tier 2 CRD, depending on which CRD is central to the area of interest. For each primary CRD, two concentric rings of CRDs were identified using a GIS proximity mapping function. CRDs that touched the primary CRD were designated as Tier 1 CRDs and CRDs that were outside but touching Tier 1 CRDs were designated as Tier 2 CRDs. Figure 2, for example, shows Primary CRD 20060 (Kansas CRD 60) and the Tier 1 and 2 CRDs that are associated with it. Each of the 304 CRDs were considered primary CRDs and their associated Tier 1 and Tier 2 CRDs were determined and were used to calculate atrazine crop application rates when AgroTrak data were not available for the CRD.

\section{Method for Estimating Atrazine Use}

Annual estimates of atrazine use on agricultural land in each county were developed using the best available pesticideuse information and crop data for each individual year, as determined by a hierarchy of decisions. This section describes the method used to develop atrazine crop application rates for DMRK surveyed and unsurveyed CRDs, develop countylevel harvested crop acreage for individual crops, and estimate annual atrazine-use by crop and county. The atrazine-use estimates derived using these methods are hereinafter referred to as EPest use estimates, while the crop application rates for

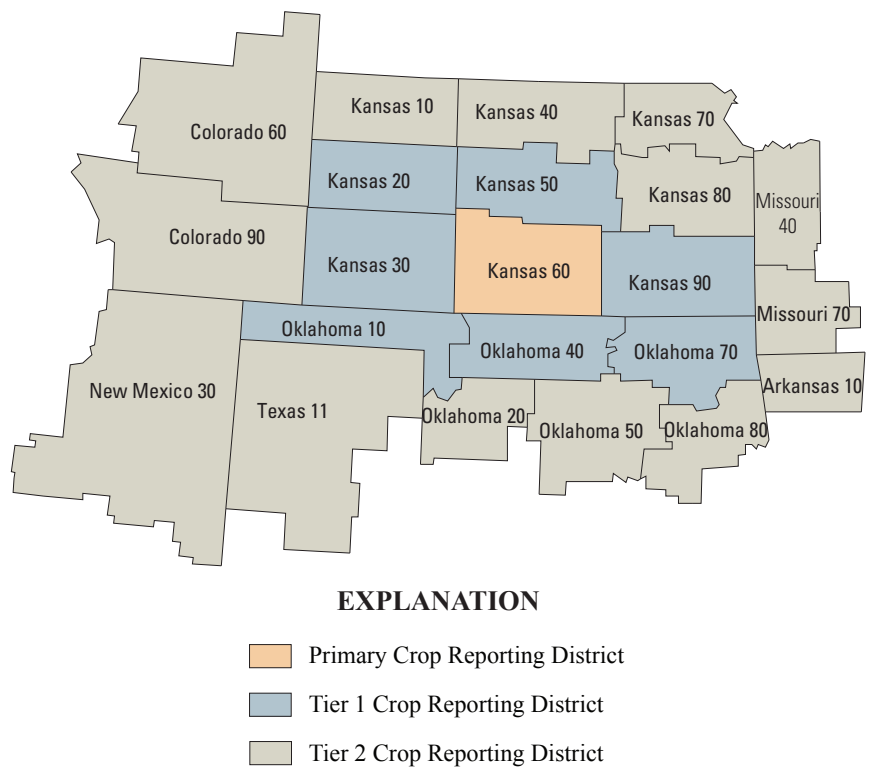

Figure 2. Example of a Primary Crop Reporting District (CRD) and surrounding Tier 1 and Tier 2 CRDs.

harvested acres in surveyed and nonsurveyed CRDs that were developed following this method are hereinafter referred to as EPest rates. The method consists of six steps for counties in all states except for California. (Atrazine-use estimates for California were derived directly from DPR-PUR atrazine-use data and are described later in this report.)

1. Compile annual harvested crop acreage for each county from the Census of Agriculture and NASS for each of the crops for which AgroTrak reported atrazine use during 1992-2007.

2. Sum annual county harvested crop acreage by CRD to produce CRD harvested crop acreage totals.

3. Calculate annual atrazine application rates per harvested acre for each crop in a CRD by dividing the total mass of atrazine for a DMRK-surveyed CRD by the harvested crop acreage total (surveyedCRD EPest rate).

4. Estimate annual atrazine application rates per harvested acre for CRDs not surveyed by DMRK using rates from adjacent (Tier 1) CRDs (Tier 1 EPest rate), when available, or from CRDs adjacent to Tier 1 CRDs (Tier 2) when Tier 1 data were incomplete (Tier 2 EPest rate).

5. Estimate regional median atrazine application rates per harvested acre for CRDs that were not surveyed by DMRK and that did not have a Tier 1 or Tier 2 crop application rate on the basis of the crop application rates from a USDA Farm Production Region for a specific year or for all years (regional EPest rate). 
6. Use the harvested acreages from step 1, the CRD crop acreage totals from (2), and application rates per harvested acre derived from steps 3,4 , or 5 to estimate atrazine use on agricultural land in each county for each year (EPest use).

\section{Compilation of Annual Harvested Crop Acreages for each County}

Annual harvested crop acreages were compiled from Census of Agriculture and NASS data for 1992 through 2007. The AgroTrak database included atrazine-use estimates for one or more years for 1992 through 2007 for 58 crops; however, a subset consisting of 16 major and minor crops and four categories of agricultural land uses - including pastureland, summer fallow, lots and farmsteads, and land set aside for conservation reserve programs - were selected and used to develop the atrazine database. Some minor crops were not included in the atrazine database because the amount of acreage for some crops, such as roots and tubers and bulb crops, was small, and compared to the mass applied to the other crops selected, reported atrazine use on these minor crops was insignificant and DMRK estimates were available for only a few years. Atrazine-use estimates were developed for the crops and agricultural land uses listed in table 2.

For Census years, harvested crop acreage data from the 1992, 1997, 2002, and 2007 Census of Agriculture were compiled for these 16 crops and four agricultural land uses. For most crops, the harvested crop acreage for a single crop category was used to calculate the crop application rate. However, for some crops, such as sorghum, the Census reported four categories of sorghum: sorghum for grain, sorghum for silage, sorghum hogged or grazed, and sorghum for syrup. To insure that all crop acres were included when calculating the overall application rate for the crop, the acreage for the four sorghum categories was summed and used to calculate the atrazine-sorghum application rate and estimate county atrazine use on sorghum. Table 2 lists the Census of Agriculture items that were summed to compute harvested crop acreage.

For non-Census years, NASS harvested acreage estimates were compiled for 14 of the major crops included in the NASS county database. NASS county harvested acreage estimates do not necessarily match acreage reported in the Census of Agriculture for the same years because NASS county acreage is based on a sample of growers and statistically derived county totals, whereas the acreage reported in the Census of Agriculture is collected from a complete enumeration of all farms producing the crop. Table 2 lists the NASS crops for which data were retrieved and used to produce harvested crop acreage totals for 1993-1996, 1998-2001, and 2003-2006.

Annual harvested acreage for each crop in each county was estimated by combining data from the Census of Agriculture for Census years with annual NASS survey data for non-Census years. For some years, harvested acres for a crop and county were not reported in either the Census or NASS annual survey data, but these data sources reported acres for other years for that crop and county. Missing years of data could be due to nondisclosure rules that prohibit publication of data when there are too few reports for a county, or in the case of NASS, when not all the same crops or states are surveyed annually. When acreage was not reported for a crop and county for a specific year (or years), but was reported for that crop and county in another year, linear interpolation between reported years for that crop and county was used to estimate acreage. The method used to fill in missing county harvested acreage requires the assumption that harvested crop acreage estimates based on Census and NASS are comparable.

Following these procedures, county harvested crop acreages were estimated for each of the years 1992 through 2007 for the crops listed in table 2. Annual CRD crop harvested acreage totals were calculated by summing the crop acreage for the counties (table 1) within each CRD.

\section{Calculation of Atrazine Application Rates for Crops in CRDs Surveyed by DMRK: Surveyed- CRD EPest Rates}

CRD crop application rates contained in AgroTrak were not used to estimate county atrazine use because these rates were developed using planted acreage. If applied to harvested crop acreage, these planted-acreage rates would, in most cases, produce total CRD use estimates that are lower than total atrazine use estimates reported in AgroTrak. This underestimation occurs because harvested acreage is typically less than planted acreage. One objective in developing the county atrazine-use database was to ensure that atrazine estimates developed using this method preserved the reported AgroTrak total mass for surveyed CRDs. Therefore, a new EPest rate was developed by dividing the surveyed mass of atrazine applied to the CRD by the harvested crop acreage estimate for the CRD to yield an atrazine application rate per harvested acre.

For each year, a new CRD crop application rate was calculated using harvested crop acreage. In some cases, the new rate was unrealistically high when compared to surveyed rates and atrazine label rates because the harvested acreage was substantially less than the planted acreage. To compensate for the difference in planted and harvested acreage, an adjustment factor was developed that effectively increased the harvested acreage in the CRD to equal the planted acreage reported in AgroTrak. Specifically, a county-CRD weighting factor by crop and year was calculated by determining the percentage of acreage for individual crops that each county contributed to the CRD's total acreage for that crop. When the sum of the harvested crop acreage totals for the counties in the CRD was less than the AgroTrak reported planted acreage, the weighting factor was applied to adjust the harvested crop acreage to the planted acreage total to apportion revised acreage values to the counties that are within the CRD. 
The new CRD crop application rate (EPest rate) was then calculated by dividing the AgroTrak report of total pounds of atrazine applied to a crop in a CRD by the revised crop acres in the CRD.

The new surveyed-CRD EPest rates were applied to the crop acreage estimates for the counties within each CRD. This produced county use estimates for all counties and years within surveyed CRDs that had reported atrazine use in the AgroTrak database. However, some CRDs may not have been surveyed by DMRK for a particular year or combination of years, even though atrazine may have been used within those CRDs, thus indirect estimates were required for these CRDs.

\section{Estimation of Atrazine Crop Application Rates for Unsurveyed CRDs: Tier 1, Tier 2, and Regional EPest Rates}

A primary objective of this study was to produce estimates of atrazine use on all reported crop acreage in the conterminous United States that likely had applications of atrazine. The AgroTrak database had atrazine survey estimates for as many as 295 of the 304 CRDs contained in the conterminous United States, but not all of these CRDs were surveyed every year between 1992 and 2007, nor was every crop surveyed during this period.

To obtain an EPest rate for the crops in a CRD not surveyed in a given year or years, a hierarchy of decision rules was developed to determine how the rate would be calculated. For a specific year and crop, the decision rules were stepped through as described below and shown in figure 3.

First, it was determined if an EPest rate(s) for a CRD not surveyed during a particular year could be calculated on the basis of EPest rates for that same year from adjacent surveyed CRDs. The proximity table (described in table 1) includes a list of Tier 1 and Tier 2 CRDs that surrounded the unsurveyed primary CRD (the primary CRD is the CRD for which an EPest rate is to be derived). This list of Tier 1 and Tier 2 CRDs was used to search for surveyed-CRD EPest rate(s) which could be used to estimate atrazine use for this primary CRD. If a surveyed-CRD EPest rate existed in one or more of the Tier 1 CRDs, then the primary CRD adopted the median value of the Tier 1 surveyed-CRD rates (Tier 1 EPest rate). If there were no surveyed-CRD EPest rates for Tier $1 \mathrm{CRD}$ rates, then surveyed-CRD EPest rates from a minimum of three Tier 2 CRDs were required to calculate the Tier 2 EPest rate as the median of qualifying CRDs.
If no Tier 1 or Tier 2 EPest rates were available, but the primary CRD had been surveyed at least once during 1992 through 2007, a regional EPest rate was estimated. To develop regional EPest rates, each $\mathrm{CRD}$ was associated with one of the 10 USDA Farm Production Regions (ig. 1), which are areas that have generally similar conditions affecting crop management, including economics, soils, and climate (U.S. Department of Agriculture, 1998). A regional EPest rate for a particular year and CRD was calculated as the median of the surveyed-CRD, Tier 1, and Tier 2 EPest rates from all of the CRDs in the Farm Production Region. Lastly, if a regional EPest rate could not be calculated for the particular year from Tier 1 or Tier 2 surveyed rates for that year, but the CRD had been surveyed at some point in time, surveyed-CRD, Tier 1, and Tier 2 rates from the entire period 1992-2007 were used to calculate a regional EPest rate (median of all CRD values), which was assigned to that CRD.

\section{Application of California DPR-PUR Data}

California PUR data were obtained from DPR (Department of Pesticide Regulation, 2009) for 1992 through 2007 to estimate county atrazine use for California. Table 3 contains a list of the 41 crops that were reported in the DPRPUR database for one or more years and the 16 crops, reported by AgroTrak for California. Although both DPR-PUR and AgroTrak include estimates for some of the same crops, DPRPUR included atrazine data for specialty crop categories not included in AgroTrak, such as strawberries, sod, tomatoes, olives, nurseries, and Bermuda grass.

Annual county estimates of atrazine use on individual crops were compiled from DPR-PUR data through a three step process: (1) summing the individual atrazine applications per crop to produce atrazine annual totals for each crop and TRS, (2) summing the atrazine annual totals for all crops in each TRS, and (3) finally summing the total atrazine use for all TRS in each county to produce the total atrazine use by county. 


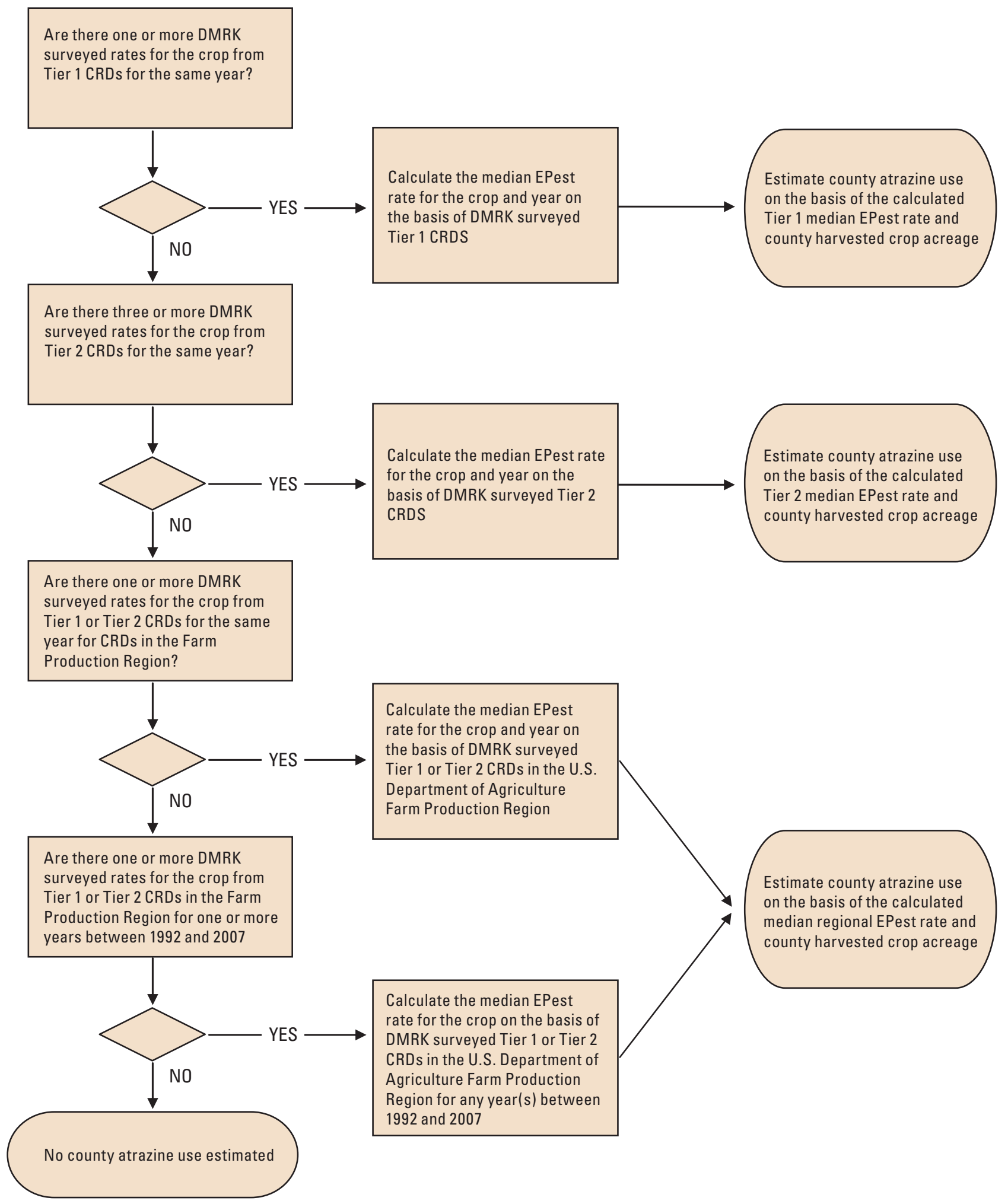

Figure 3. Summary of the decision process followed to estimate EPest rates and EPest use for unsurveyed Crop Reporting Districts (CRD). DMRK, Doane Marketing Research-Kynetec. 


\section{Results and Discussion}

The atrazine-use database produced from the methods described above includes county-level estimates of the mass of atrazine applied to agricultural crops from 1992 through 2007. Annual county estimates of atrazine used on as many as 16 major and minor crops and four agricultural land uses were made for as many as 2,966 counties in one or more years for 1992 through 2007 (fig. 3). Estimates for some crops are not available for every state or year, and estimates for some crops, such as avocados, strawberries, and walnuts, were reported by DPR-PUR and are available for California only. Atrazine-use estimates for individual crops were totaled for each county and year and are available from $\mathrm{http}$ ://water.usgs.gov/nawqa/pnsp/ data/atrazine use 1992 2007.dat

The total amount of atrazine used annually in the conterminous United States fluctuated between a low of about 66 million pounds in 2006 to a high of about 82 million pounds reported in 1996 (fig. 4). Some of the fluctuation may be related to changes in AgroTrak survey methods over time, in addition to changes in crop acres harvested or application rates. Between 1992 and 1997, DMRK collected data on all crops that were part of a farm operation, but in 1998, DMRK began to survey and collect data for major crops and selected specialty crops across all states so that data for several minor crops were no longer collected. Then in 2005, DMRK further modified their survey design so that major crops and specialty crops were surveyed only in those states that accounted for 80 percent of the national crop acreage as determined from USDA acreage estimates from the previous year. However, for some specialty crops, additional states were surveyed so that more than 80 percent of some specialty crop acres were surveyed.

Figure 4 shows the annual proportional distribution of atrazine use on corn, sorghum, sugar cane, and other crops. The first three are major row crops for which atrazine is most heavily used to control weeds; "other crops" includes fallow, pasture, hay, wheat, and other minor crops. More than 80 percent of the estimated atrazine use was on corn, followed by sorghum, which accounted for about 7 to 10 percent of use, and sugar cane, which accounted for between 1 and 3 percent of atrazine use in the conterminous United States. Atrazine use on other crops accounted for between 1 and 3 percent of total annual use.

Between 96 and 99 percent of the estimated atrazine used on all crops in the conterminous United States each year was accounted for by EPest rates derived from surveyed CRDs

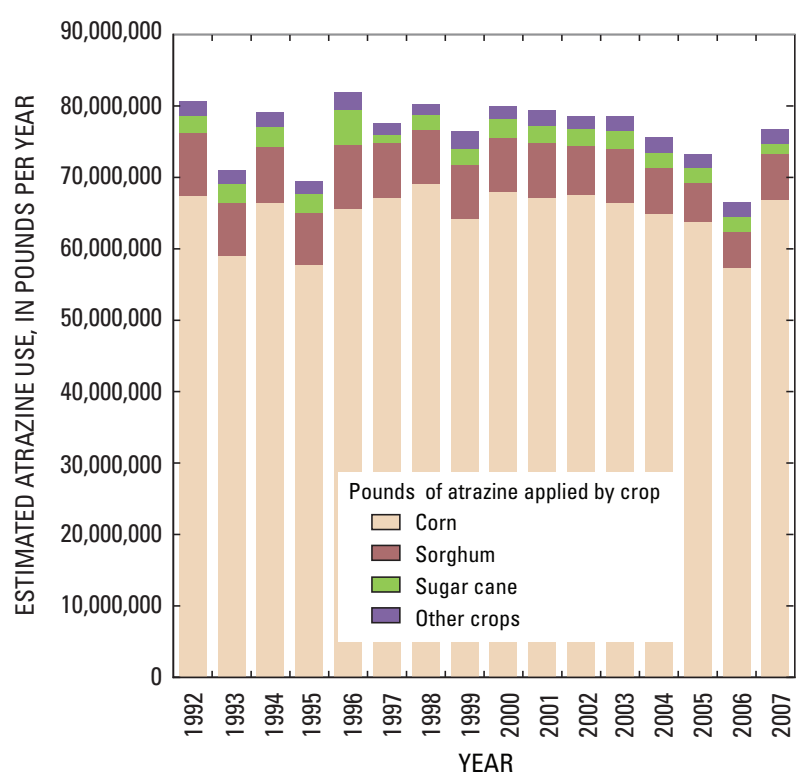

Figure 4. National use of atrazine on major crops and other crops, 1992-2007.

(fig. 5A). Regional EPest-rates calculated across the 16-year period (1992-2007) added approximately 1 to 2 percent to the national total in any given year, and Tier 1 EPest rates added 1 percent or less to the annual national total in most years.

Tier 2 EPest rates contributed the least to the national total in any year and California DPR-PUR added a small fraction of a percent, or about 22 to 40 thousand pounds annually to the national total. Most estimates of atrazine used on the major crops corn, sorghum, and sugar cane between 1992 and 2005 were based on surveyed rates; less than 1 percent was derived using EPest rates (fig. 5B). However, in 2006 and 2007, about 1 percent of atrazine use was estimated using Tier 1 or regional EPest rates for a specific year. Extrapolation methods were used more frequently to estimate use on minor crops with 26-63 percent of atrazine use on these crops estimated using Tier 1, Tier 2, or regional EPest rates (fig. 5C). EPest rates for surveyed CRDs were not available for many of the minor crops because most of these crops were not surveyed every year, and some CRDs or crops were surveyed only once during the 16 year period. Of the extrapolation methods used, regional EPest rates calculated from the data for the 16 year period contributed the greatest amount to estimates of atrazine use on minor crops. 


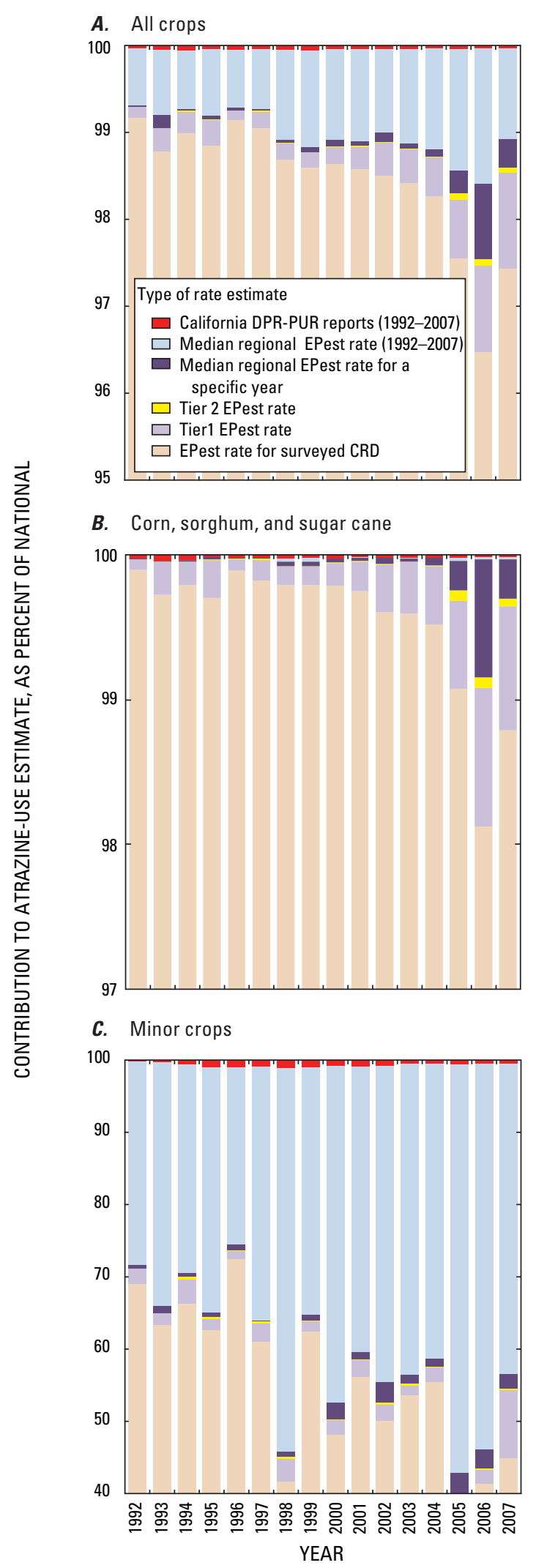

Figure 5. Contribution of atrazine use by estimation method $(A)$ on all crops, $(B)$ on corn, sorghum, and sugar cane, and $(C)$ on minor crops, 1992-2007.
National patterns of atrazine-use intensity and the percentage of total county atrazine use derived from Tier 1 , Tier 2, or regional EPest rates are shown for 1992-2007 in figure 6. Atrazine-use intensity expressed as an annual average rate of atrazine use in a county was calculated by dividing the total mass of atrazine applied in each county by the square miles of land in the county. A frequency distribution of county atrazine-use intensity was used to divide the atrazine-use intensity data into five classes (quintiles) so that each class represented 20 percent of the use-intensity values. These five classes were then used to show atrazine-use intensity on agricultural land in each county as determined from a 2001 version of the National Land Cover Dataset (NLCD) (figure 6). To facilitate comparisons of use patterns across all geographic regions and years, use-intensity intervals shown in figure 6 were based on atrazine use for 1998 - the year that had the maximum amount of atrazine used nationally. As these maps show, the greatest concentration of atrazine-use was in the areas where corn and sorghum were the primary crops, including the Corn Belt, parts of the Northeast, the Mid-Atlantic States, and the Texas-New Mexico area. Outside of these areas, atrazine use was also estimated for one or more counties in each state throughout the 16-year period, but the estimates were much lower. Using Tier 1, 2, and regional EPest rates increased the percentage of counties outside the Corn Belt for which atrazine use could be estimated; 80 percent or more of estimates for counties in some western states were derived from Tier 1, Tier 2 and regional EPest rates. 
1992

Total use: $80,774,073$ pounds

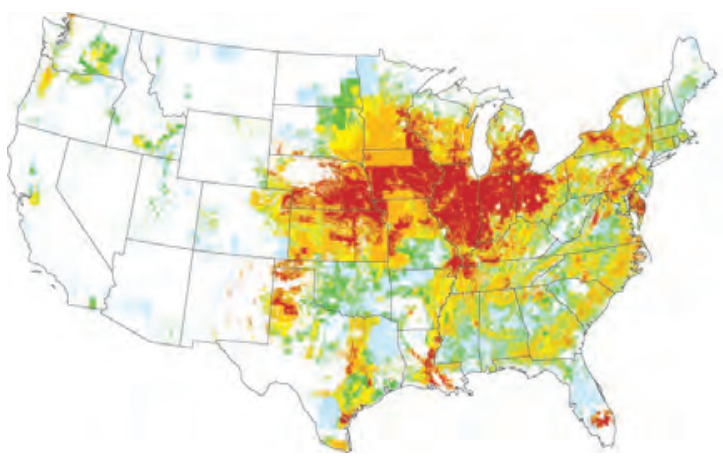

1993

Total use: $70,898,659$ pounds

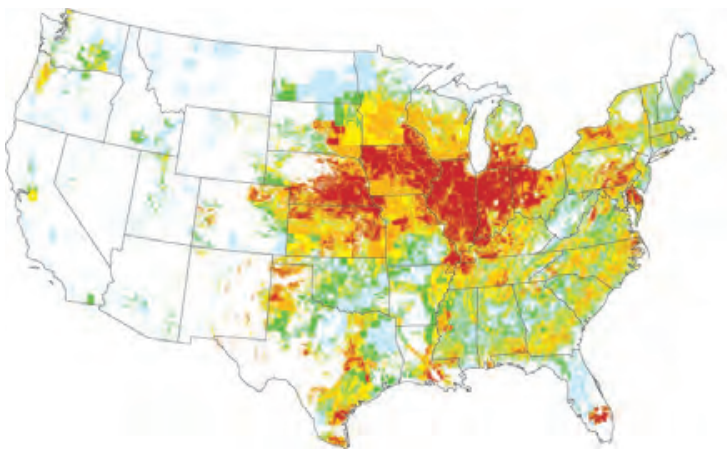

1994

Total use: $79,217,945$ pounds

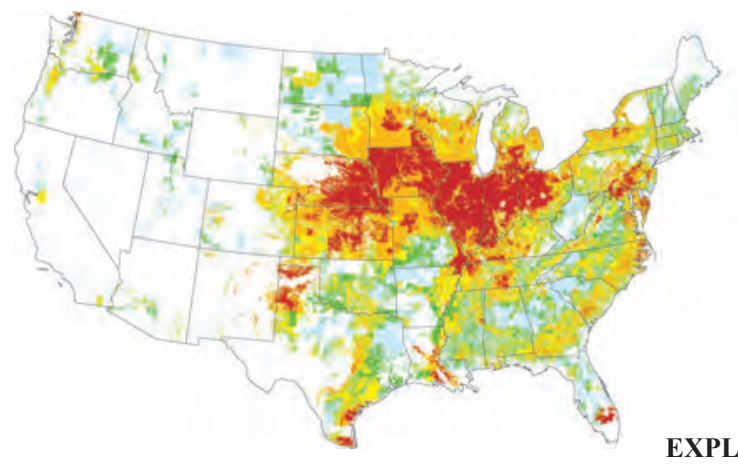

EXPLANATION

Estimated use intensity, in pounds per square mile per year. Quintiles were calculated on the basis of 1998 atrazine use.
$\square>0-0.13$
$>0.13-0.84$
$>0.84-3.3$
$>3.3-10$
$>10$

Distribution of use in non-surveyed CRDs

(0.86 percent of national total)

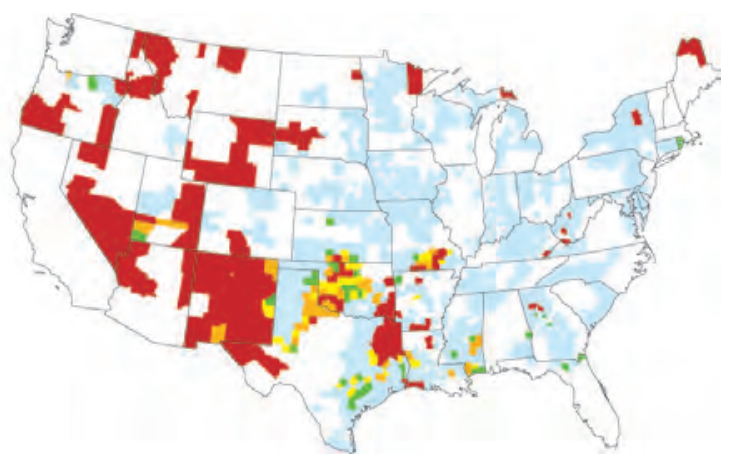

Distribution of use in non-surveyed CRDs

(1.17 percent of national total)

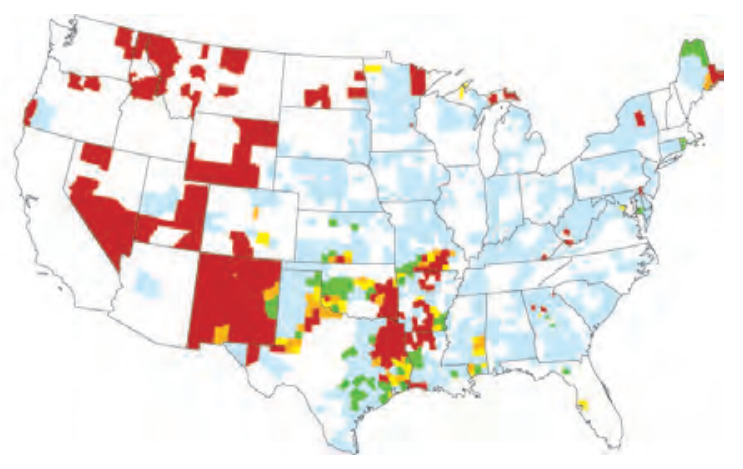

Distribution of use in non-surveyed CRDs

(1.04 percent of national total)

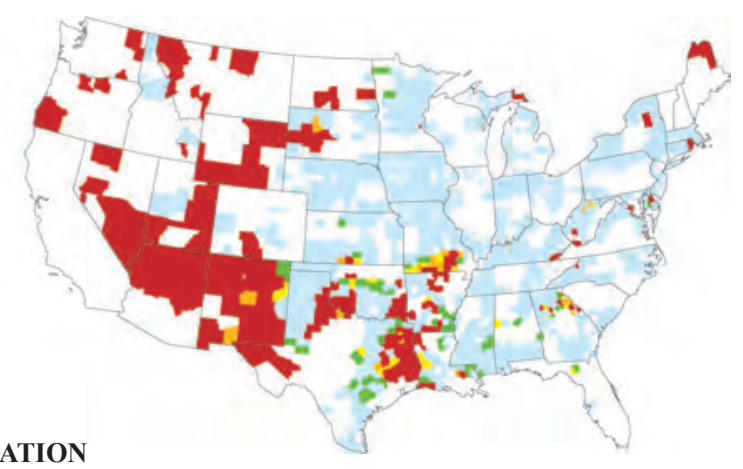

Proportion of atrazine use estimated using Tier 1, 2 or regional median rates, as percentage of county total
$\square$ 1-20
$\square 21-40$
$\square$ 41-60
$\square 61-80$
$>80$

Figure 6. Distribution of atrazine use and the proportion estimated using an unsurveyed Crop Reporting District (CRD) rate, 1992-2007. 


\section{5}

Total use: $69,471,038$ pounds

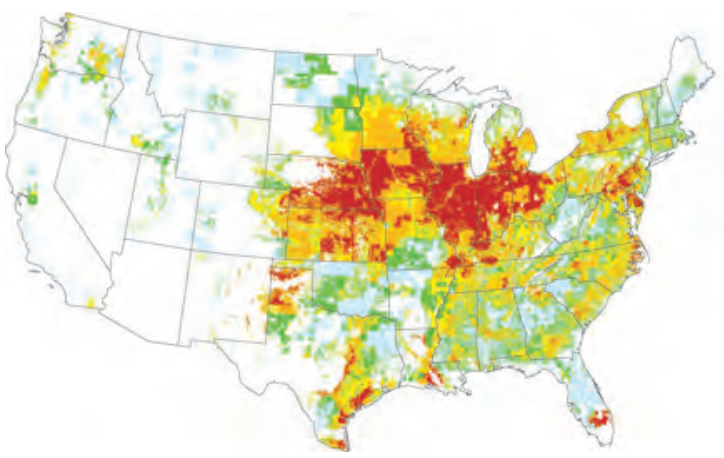

1996

Total use: $81,974,543$ pounds

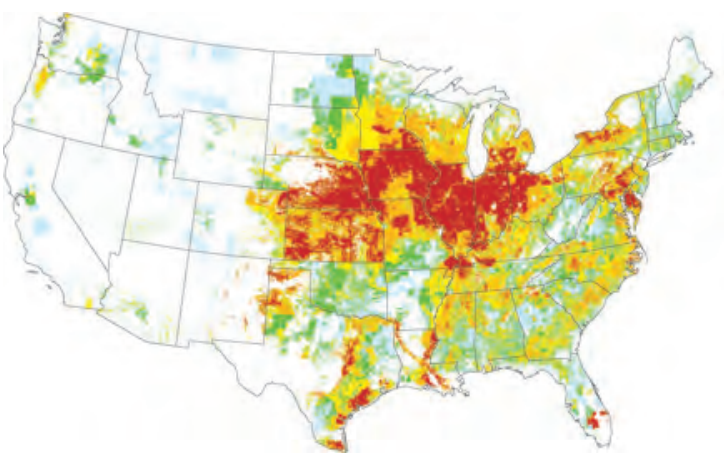

1997

Total use: $77,637,252$ pounds

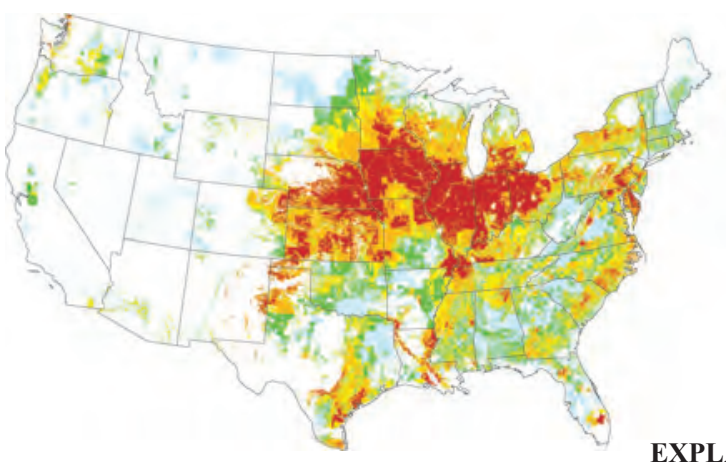

Estimated use intensity, in pounds per square mile per year. Quintiles were calculated on the basis of 1998 atrazine use.

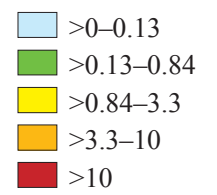

Figure 6. Continued.
Distribution of use in non-surveyed CRDs

(1.21 percent of national total)

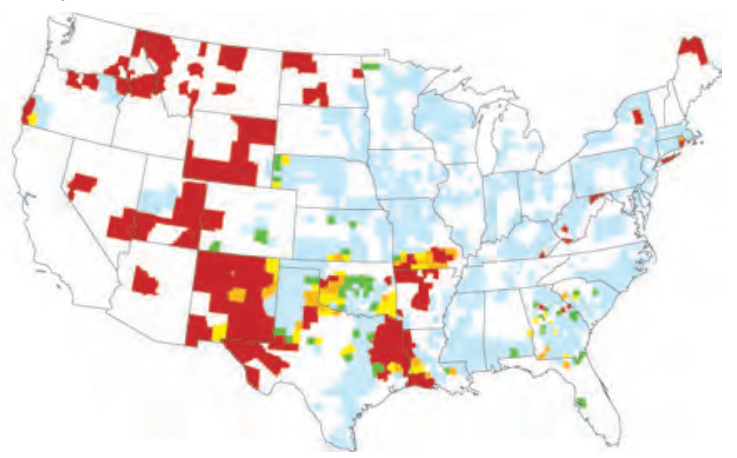

Distribution of use in non-surveyed CRDs (0.89 percent of national total)

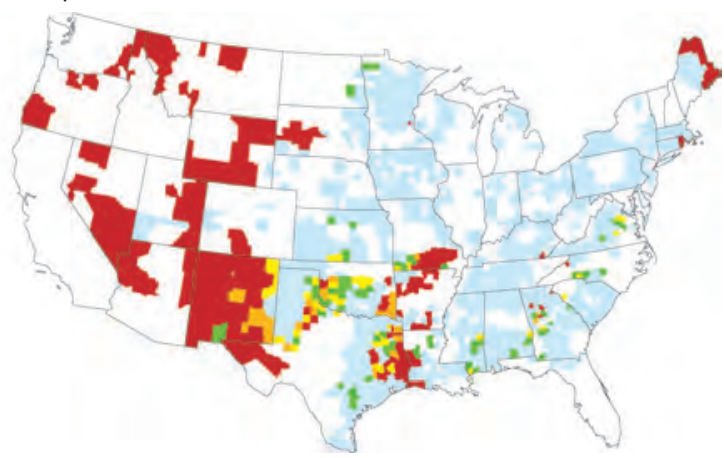

Distribution of use in non-surveyed CRDs (0.98 percent of national total)

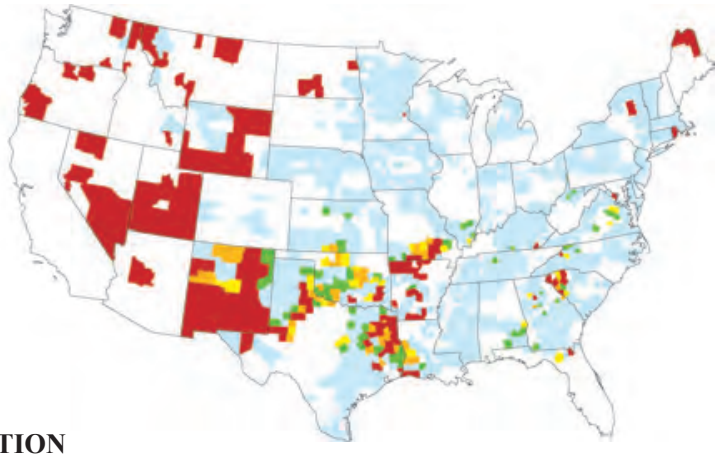

Proportion of atrazine use estimated using Tier 1, 2 or regional median rates, as percentage of county total

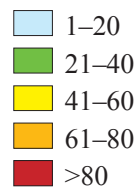


Total use: $80,239,436$ pounds

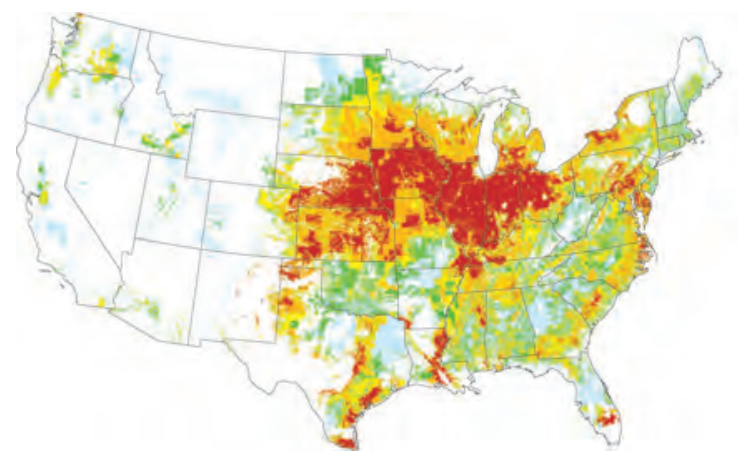

1999

Total use: $76,335,597$ pounds

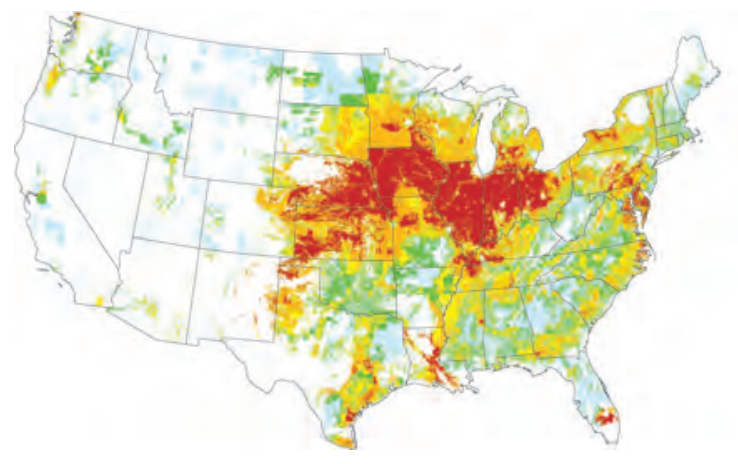

2000

Total use: $79,895,462$ pounds

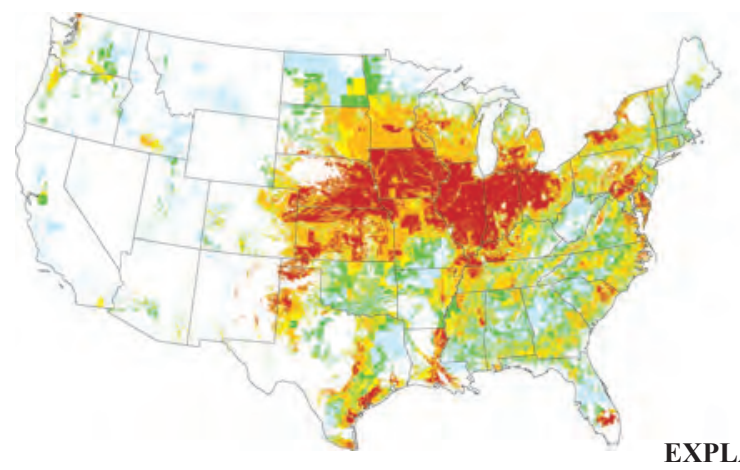

EXPLANATION

Estimated use intensity, in pounds per square mile per year. Quintiles were calculated on the basis of 1998 atrazine use.

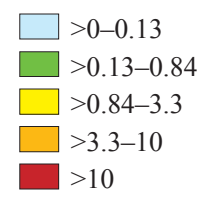

Figure 6. Continued.
Distribution of use in non-surveyed CRDs

(1.24 percent of national total)

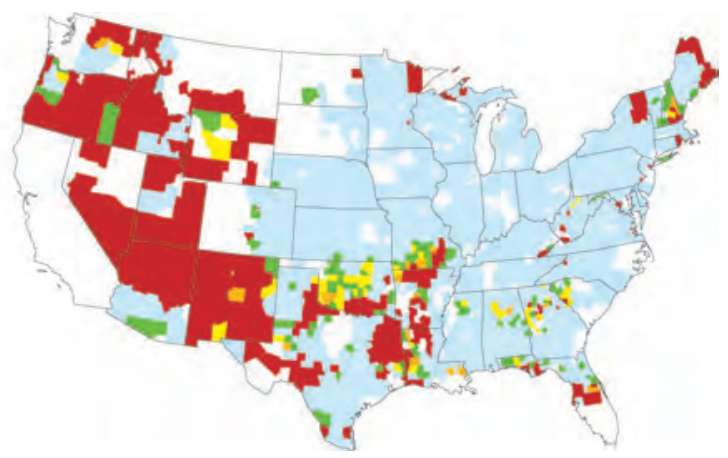

Distribution of use in non-surveyed CRDs

(1.32 percent of national total)

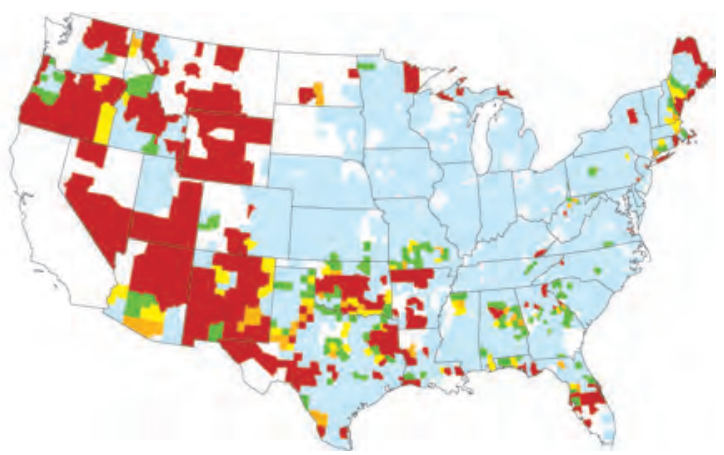

Distribution of use in non-surveyed CRDs

(1.30 percent of national total)

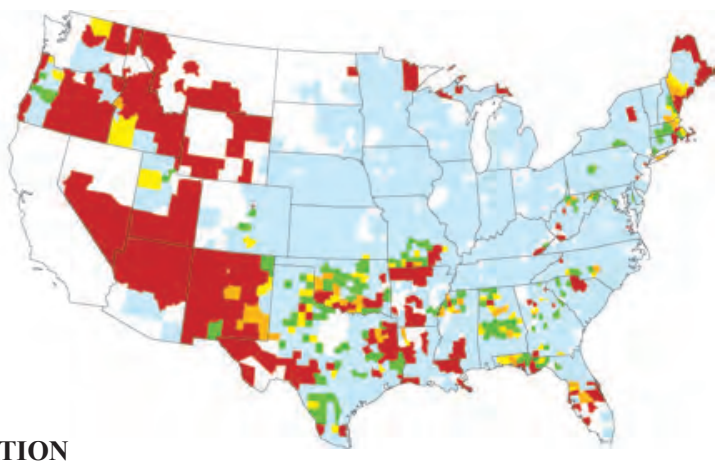

Proportion of atrazine use estimated using Tier 1, 2 or regional median rates, as percentage of county total

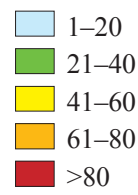


2001

Total use: $79,362,390$ pounds

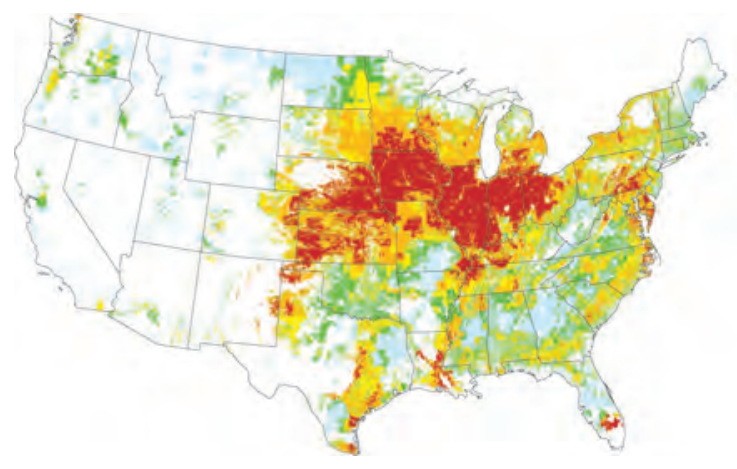

Distribution of use in non-surveyed CRDs

(1.37 percent of national total)

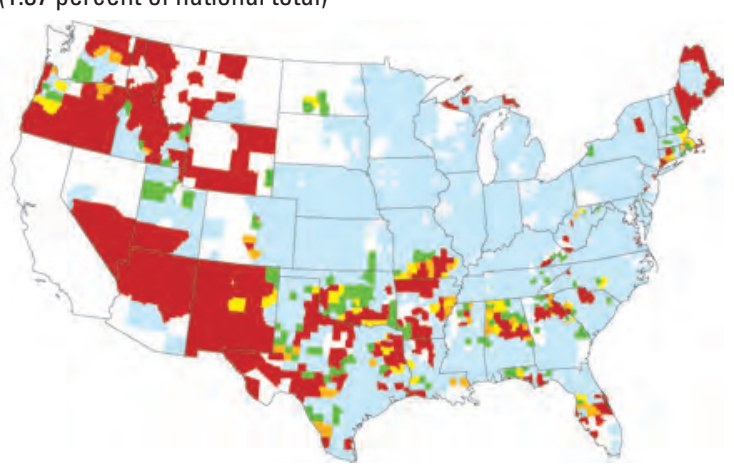

2002

Total use: $79,362,390$ pounds

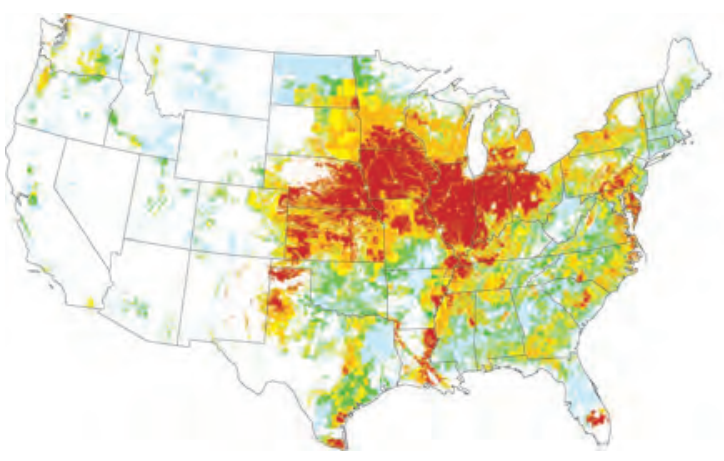

2003

Total use: $78,469,933$ pounds

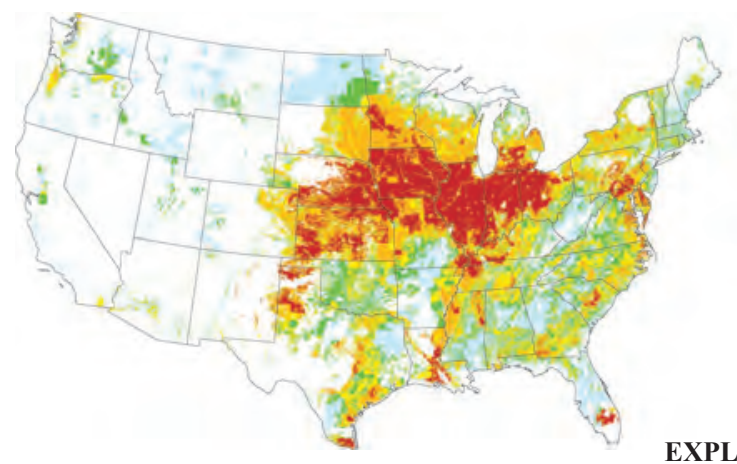

EXPLANATION

of use in non-surveyed CRDs

(1.54 percent of national total)

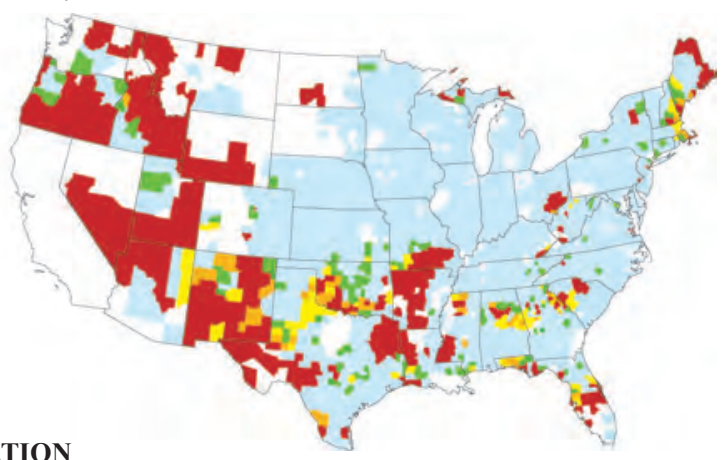

Proportion of atrazine use estimated using Tier 1, 2 or regional median rates, as percentage of county total

square mile per year. Quintiles were calculated on the basis of 1998 atrazine use.
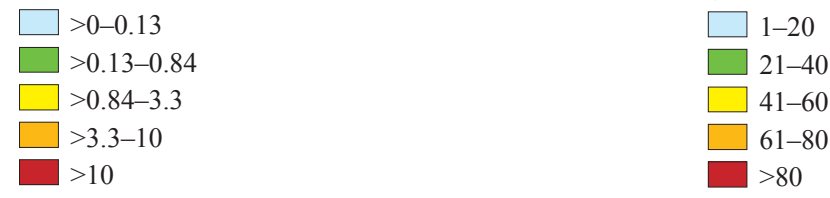

Figure 6. Continued. 


\section{4}

Total use: $75,600,508$ pounds

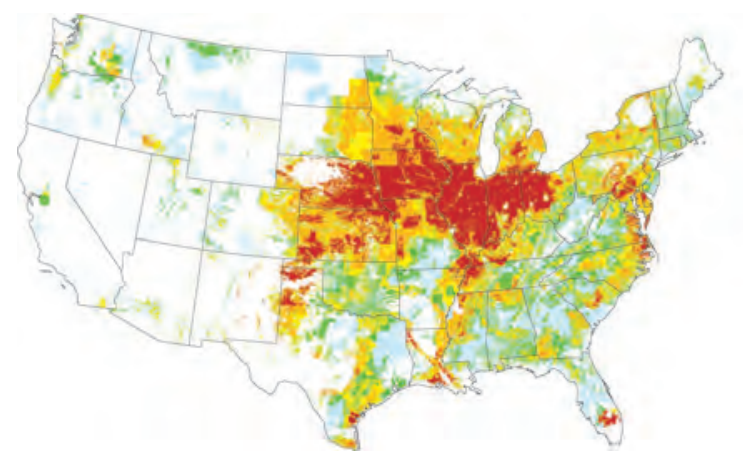

Distribution of use in non-surveyed CRDs

(1.69 percent of national total)

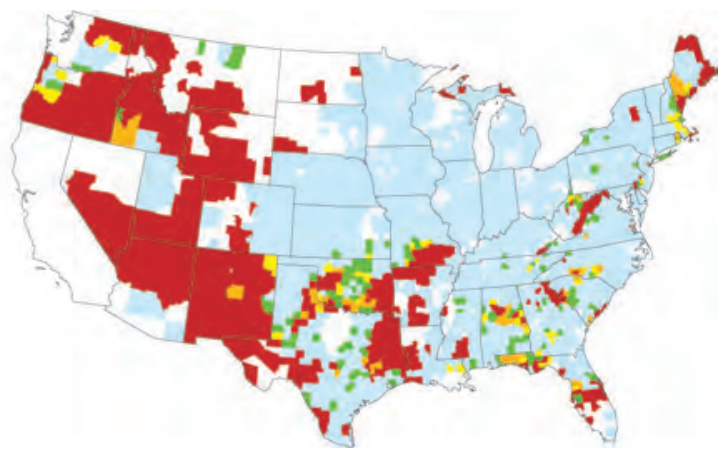

2005

Total use: $73,378,825$ pounds

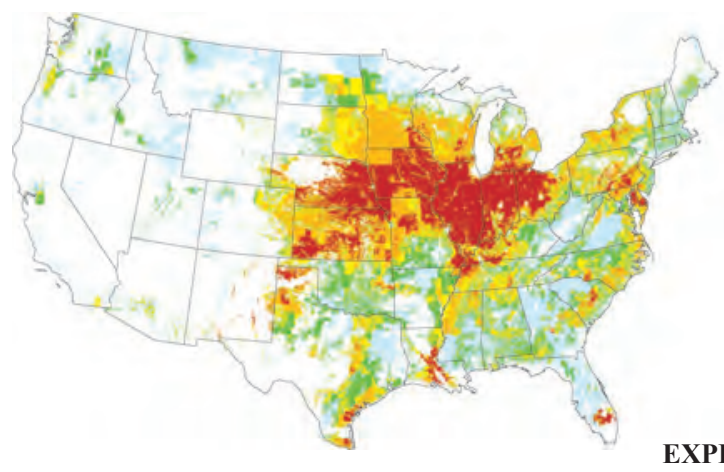

EXPLANATION

Distribution of use in non-surveyed CRDs (2.66 percent of national total)

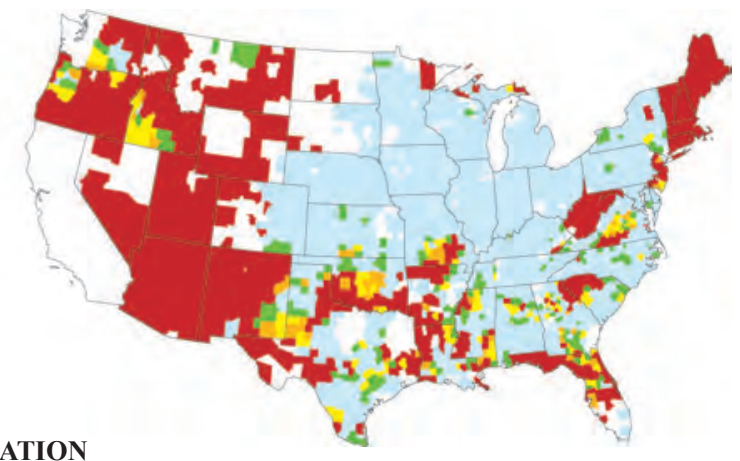

Estimated use intensity, in pounds per square mile per year. Quintiles were calculated on the basis of 1998 atrazine use.

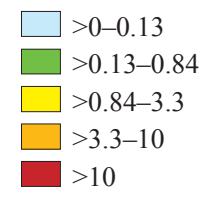

Figure 6. Continued.
Proportion of atrazine use estimated using Tier 1, 2 or regional median rates, as percentage of county total
$1-20$
21-40
41-60
61-80
$>80$ 
2006

Total use: $66,775,230$ pounds

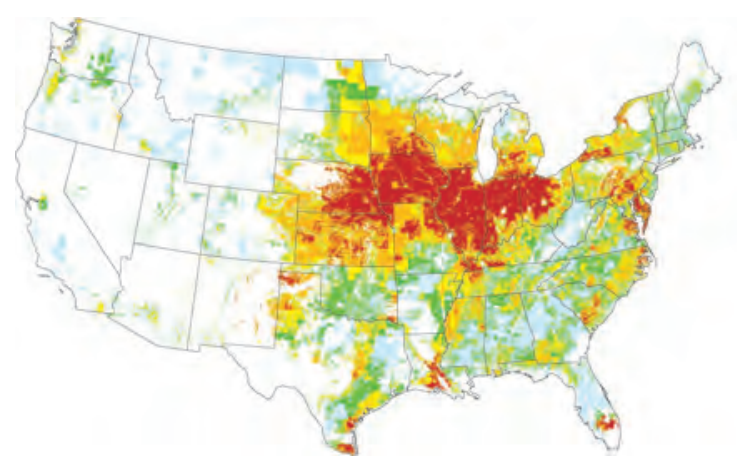

Total use: $76,891,310$ pounds

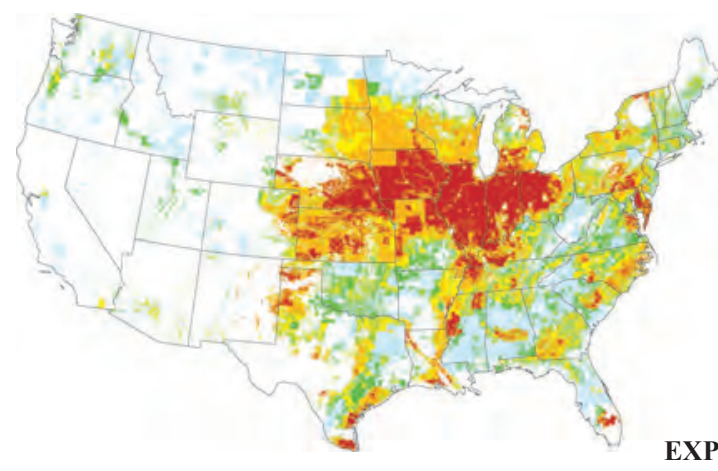

Distribution of use in non-surveyed CRDs

(3.75 percent of national total)

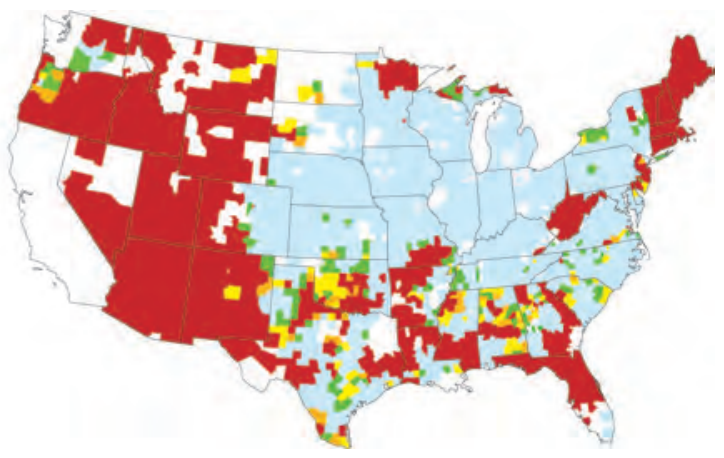

2007

Distribution of use in non-surveyed CRDs

(2.73 percent of national total)

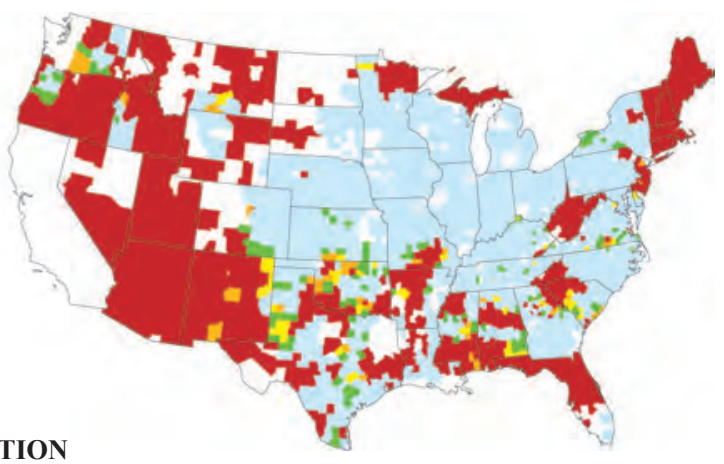

Estimated use intensity, in pounds per square mile per year. Quintiles were calculated on the basis of 1998 atrazine use.

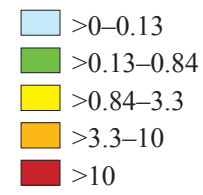

Proportion of atrazine use estimated using Tier 1, 2 or regional median rates, as percentage of county total

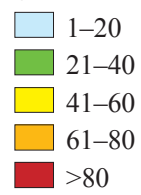

Figure 6. Continued. 
The national maps show broad patterns of atrazine use, but do not show differences in use patterns among the crops. The regional patterns in the intensity of atrazine use on corn and the source application rate that was used to estimate county-level atrazine use in 1992, 1997, 2002, and 2007 are shown in figure 7. Within the areas of primary corn cultivation, EPest rates were used almost exclusively. Tier 1, Tier 2, or regional EPest rates were used in areas that have a smaller proportion of the nation's corn acreage and consequently where AgroTrak had fewer crop surveys. Nationally, the mass derived from Tier 1, Tier 2, or regional EPest rates accounted for 1 percent or less of the total use on corn in CRDs not surveyed. However, these rates contributed more than 80 percent of the atrazine total for corn in some states. For example, an EPest rate for corn existed for only one CRD in Nevada in 2001, but atrazine use on corn was estimated in one or more counties every year from 1992 to 2007 on the basis of Tier 1, Tier 2, or regional EPest rates for adjacent areas or years. Similarly, for some years, EPest rates for atrazine on corn were available for only one or two surveyed CRDs in Arizona, Idaho, Montana, Connecticut, Vermont, and Massachusetts, but Tier 1, Tier 2, or regional EPest rates could be calculated and used to estimate atrazine use in all counties in the state that reported corn acreage. The use of Tier 1, Tier 2, or regional EPest rates from another year or multiple years assumes that atrazine application rates are fairly consistent within a localized region.

Comparing the maps of atrazine-use intensity for corn from 1992 to 2007 illustrates the effect of changes in DMRK survey methods (fig.7). As mentioned earlier, the survey method was modified in 2005 for major crops and follows an 80-20 rule that targets geographic areas that account for 80 percent of the nation's crop acres and from within these areas 15 to 20 percent of the corn acres are surveyed. The survey results were then expanded to represent the total acres and management practices within a CRD. Implementing the 80-20 rule resulted in eliminating some states from the corn survey that had previously been surveyed, such as Arizona, Florida, and Maine. Unless a Tier 1, Tier 2, or regional rate for the specific year or a regional rate from a different year is available, atrazine use on corn will not be accounted for in such states after 2004. If in a future year corn acreage in the state increases enough to account for 80 percent of national corn acreage, the state can be added back into the AgroTrak survey of corn.

Atrazine use for sorghum production ranked second and equaled about 10 percent of the total atrazine applied to corn. Sorghum is drought tolerant and is not managed as intensively as corn. Most sorghum acreage is located in the High Plains and the Lower Mississippi area where the majority of AgroTrak sorghum surveys were conducted. Sorghum is also grown in several other regions of the country, including the Mid-Atlantic States. Fewer AgroTrak surveys were done within the Mid-Atlantic States; therefore half of the atrazine use estimated in this region was based on Tier 1, Tier 2, or regional EPest rates. Beginning in 2001, sorghum acreage decreased in the Mid-Atlantic States and consequently fewer AgroTrak surveys were conducted there, so that a larger proportion of atrazine use on sorghum was calculated using EPest rates for unsurveyed CRDs. By 2005, the Mid-Atlantic States were dropped from sorghum surveys because sorghum acreage in these states did not meet the 80-percent criterion for crop acreage. Because crop acreage is one of the major criteria used to allocate AgroTrak crop surveys, changes in crop acreage determine which regions and states are included in a crop survey. In addition, if no Tier 1, Tier 2, or regional EPest rates are available from adjacent states or from other years for a specific crop, atrazine use for that crop cannot be estimated even though the Census or NASS reported crop acreage and atrazine may have been applied. The 80 -percent rule guarantees that some portion of the crop acreage in primary production areas will be surveyed, but if the crop is grown outside of the radius of Tiers 1 and 2, or if a CRD has never been surveyed and therefore does not have a rate, then pesticide use for that crop will not be accounted for using the method developed to estimate county-level pesticide use.

The amount of atrazine applied to sugar cane is third highest among the agricultural crops treated with atrazine. AgroTrak reported atrazine use on sugar cane in four states: Florida, Georgia, Louisiana, and Texas. Using DMRK surveyed CRD atrazine-use estimates for sugar cane and harvested crop acreage from the Census and NASS, atrazine application rates for sugar cane were calculated for only 3 of 4 states reported by AgroTrak because county-level sugar cane acreage was not published for Georgia. Some specialty crops, such as sugar cane, may be grown on only a few farms in a county, making it possible to connect a specific farm operation with reported crop acreage. The harvested acres may represent a large portion of the total acres grown for the crop in the CRD, but the harvested acreage may not be reported for some counties and years because of USDA nondisclosure rules that are designed to protect information about individual growers. The potential effect of unreported or nondisclosed county crop acreage is two-fold. First, crop application rates may be unrealistically high if the acreage used to calculate the rate is too low compared to the surveyed acres that AgroTrak estimated or second, as in the case of Georgia, atrazine use for a state or CRD may not be estimated even though AgroTrak reports atrazine use for the specific crop. Our method takes into account the first condition - that of under-reported crop acreage - by adjusting the county harvested acreage used to calculate the rate based on AgroTrak surveyed CRD acreage. If acreage for a crop is not reported at all, however, there is no way to make this adjustment and atrazine use for the crop will not be estimated. 
1992

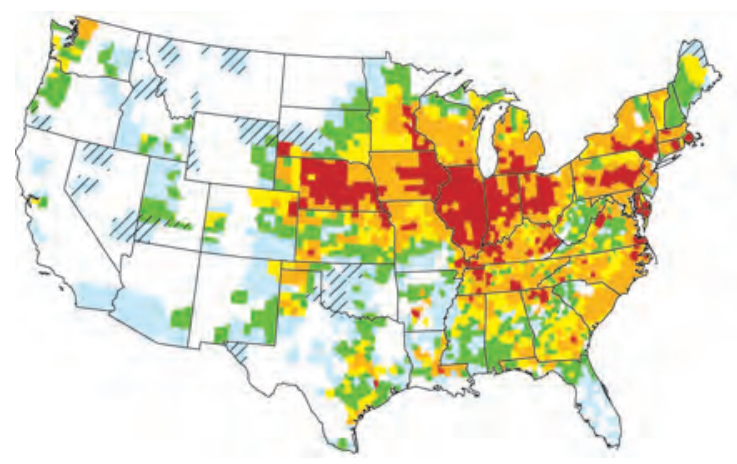

1994

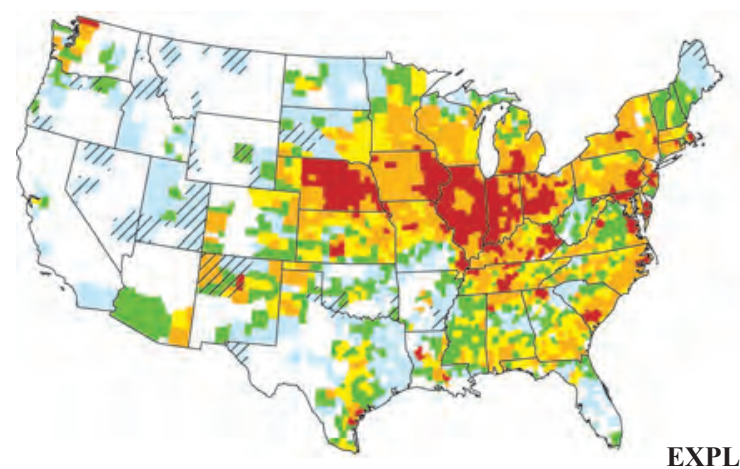

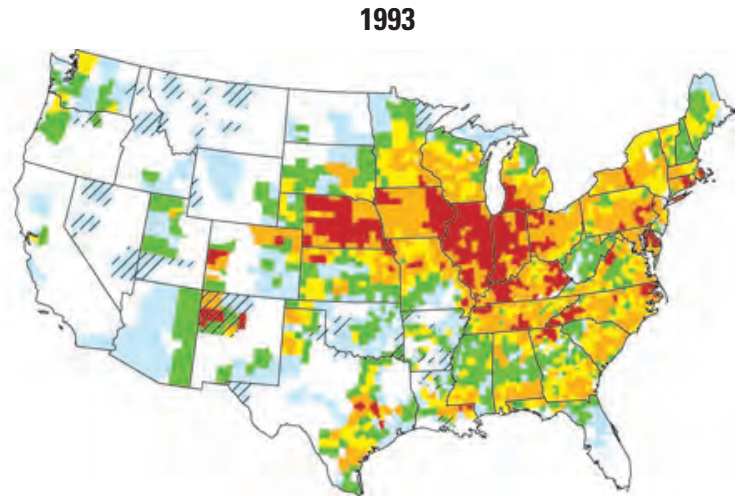

1995

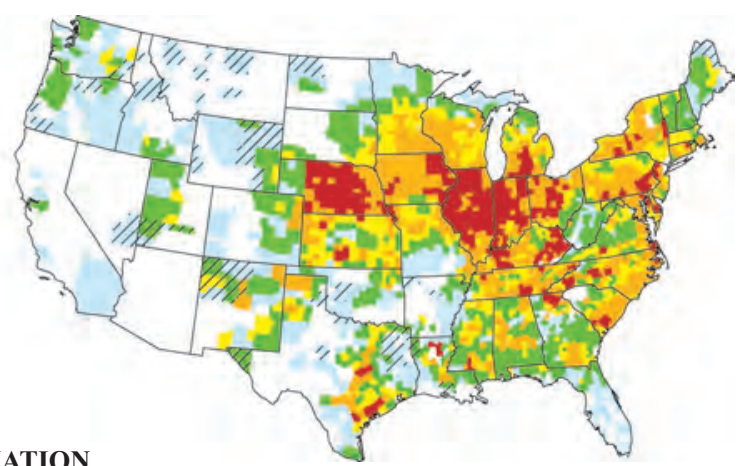

Estimated atrazine use intensity on corn, in pounds per square mile per year. Quintiles were calculated on the basis of 2007 atrazine use on corn.

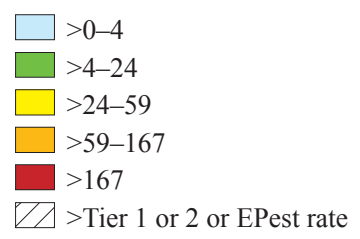

Figure 7. Distribution of atrazine use on corn and estimates derived from an unsurveyed Crop Reporting District (CRD) rate,1992-2007. 
1996

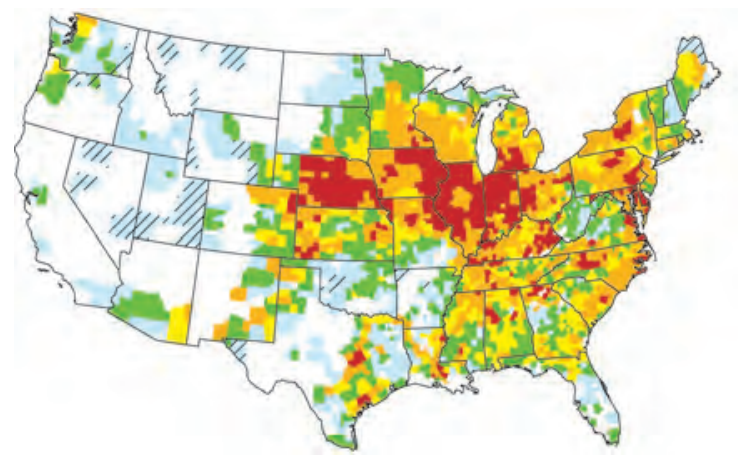

1998

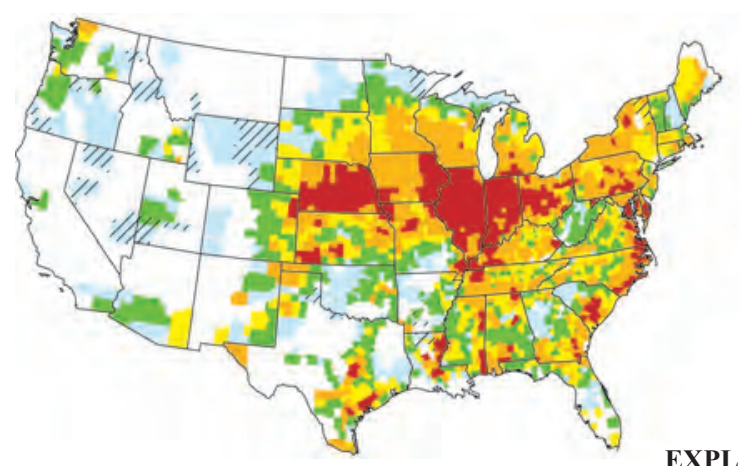

1997

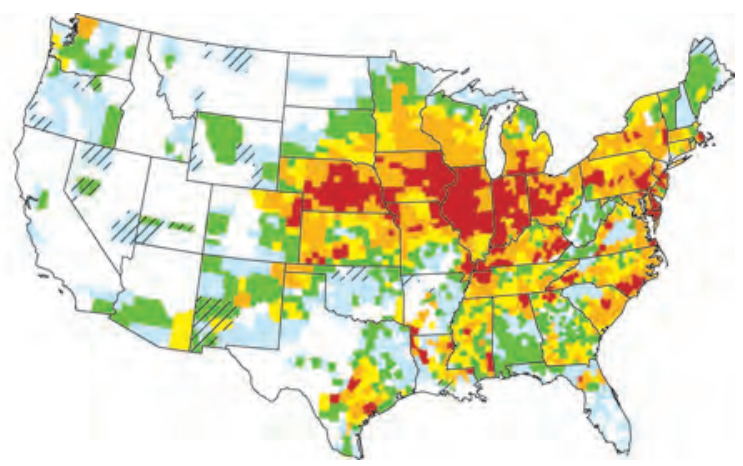

1999

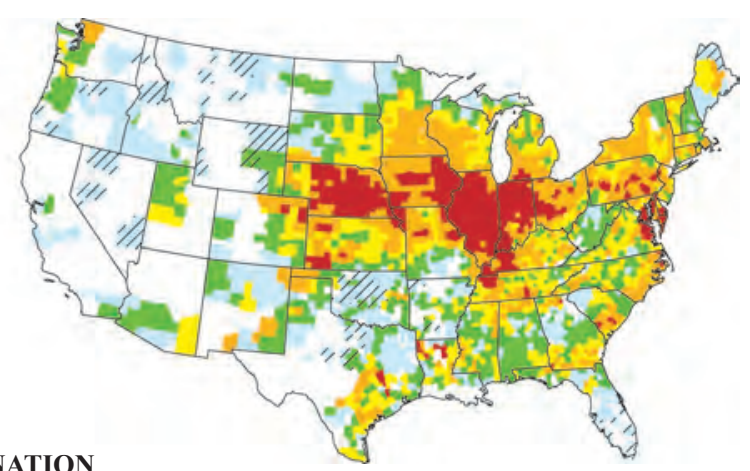

Estimated atrazine use intensity on corn,

in pounds per square mile per year. Quintiles were calculated on the basis of 2007 atrazine use on corn.

$\begin{aligned} \square & >0-4 \\ \square & >4-24 \\ \square & >24-59 \\ \square & >59-167 \\ \square & >167 \\ \square \square & >\text { Tier } 1 \text { or } 2 \text { or EPest rate }\end{aligned}$

Figure 7. Continued. 
2000

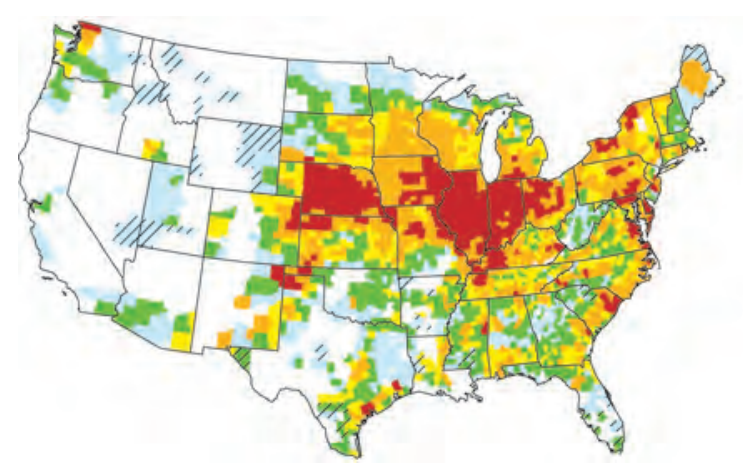

2002

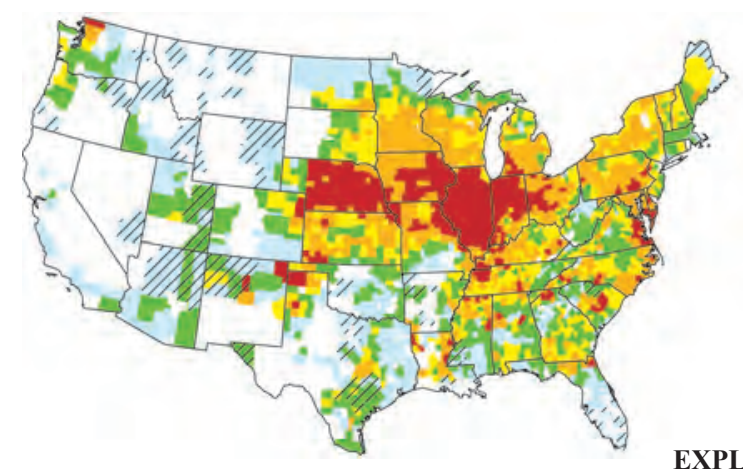

EXPLANATION
2001

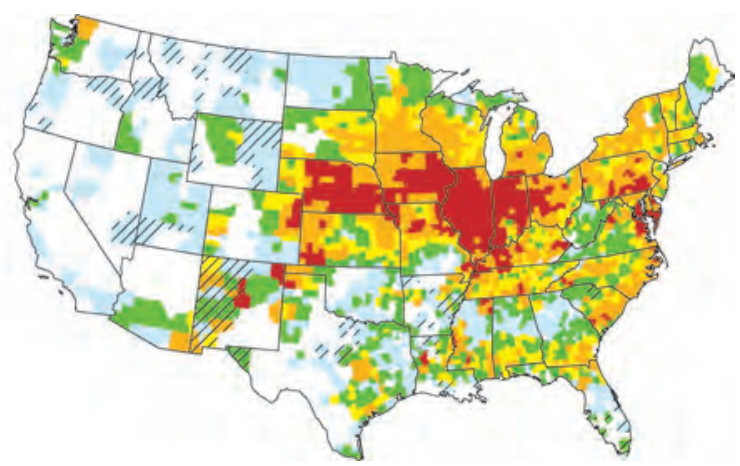

2003

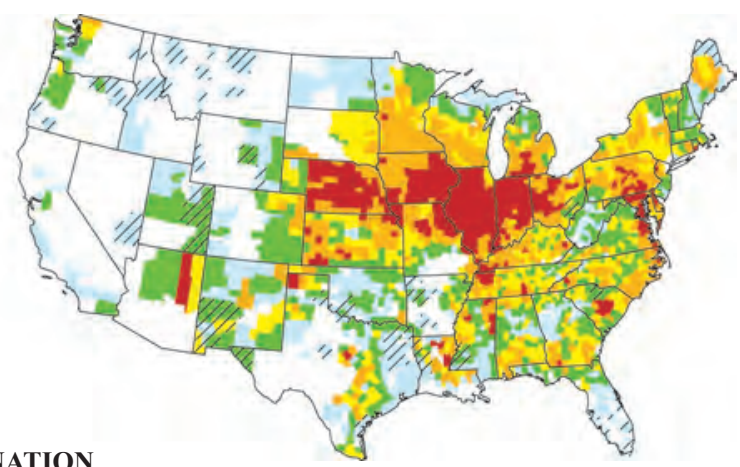

Estimated atrazine use intensity on corn, in pounds per square mile per year. Quintiles were calculated on the basis of 2007 atrazine use on corn.

$\begin{aligned} \square & >0-4 \\ \square & >4-24 \\ \square & >24-59 \\ \square & >59-167 \\ \square & >167 \\ \square Z \Delta> & >\text { Tier } 1 \text { or } 2 \text { or EPest rate }\end{aligned}$

Figure 7. Continued. 
2004

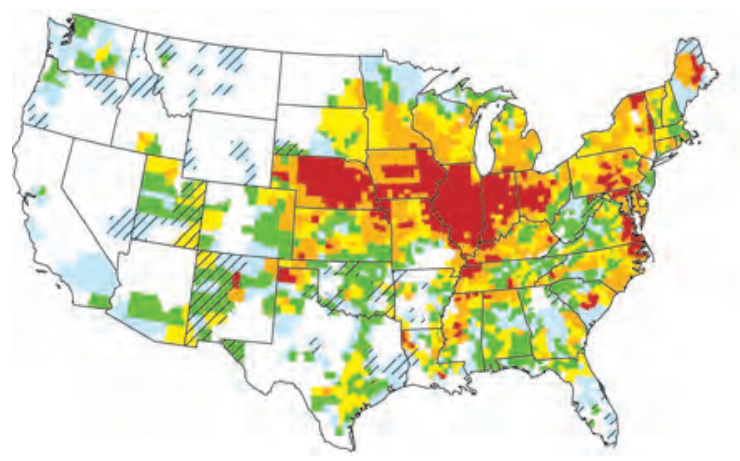

2006

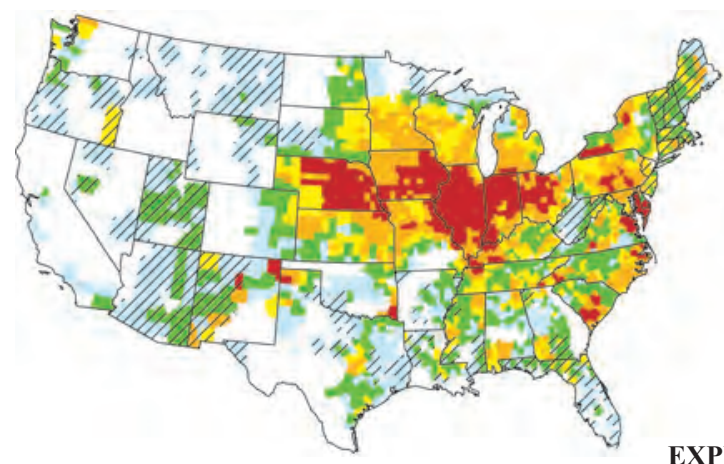

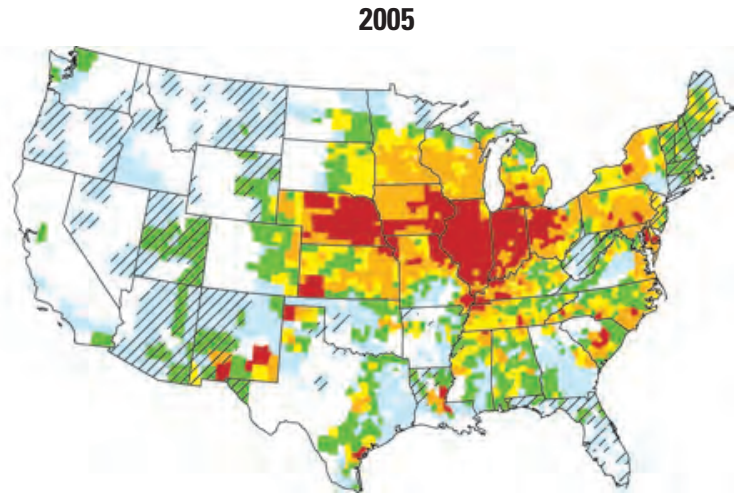

2007

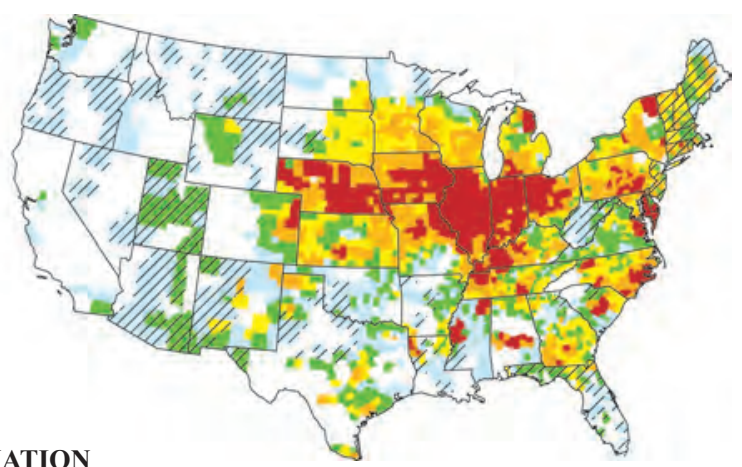

Estimated atrazine use intensity on corn,

in pounds per square mile per year. Quintiles were calculated on the basis of 2007 atrazine use on corn.

$\square>0-4$
$\square>4-24$
$\square>24-59$
$\square>59-167$
$\square>167$
$\square \triangle>$ Tier 1 or 2 or EPest rate

Figure 7. Continued. 
As these examples illustrate, the atrazine-use estimation method developed for this study is dependent on county-level harvested crop acreage reported by the USDA sources, Census or NASS, to calculate a crop application rate and allocate the pesticide mass applied to crop acres in a county reported in AgroTrak. If county harvested acreage is not disclosed by the USDA, the crop application rate calculated may be unrealistically high or AgroTrak pesticide-use reports for a crop cannot be allocated to crop acreage in the county. In order to minimize the effect of unreported crop acreage, county crop acres were estimated for some years by interpolating crop acreage for a missing year by using reported crop acres from other years. The acreage interpolation method accounted for 70 to 79 percent of the acres used to estimate atrazine use in non-Census years and accounted for 4 to 9 percent in Census years (fig. 8). Crops not reported in the NASS county database, such as pasture and summer fallow, accounted for the largest proportion of total acres derived from the interpolation method used for non-Census years. For example, summer fallow acreage during non-Census years was derived solely from interpolating county acreage for Census years. Likewise, as much as 42 percent of the corn acreage and as much as 82 percent of sorghum acreage was interpolated because NASS does not report acreage for all sorghum categories. For example, total sorghum acreage used to develop crop application rates included acreage for all categories of sorghum reported by the Census of Agriculture including sorghum for grain, silage, and syrup; however,

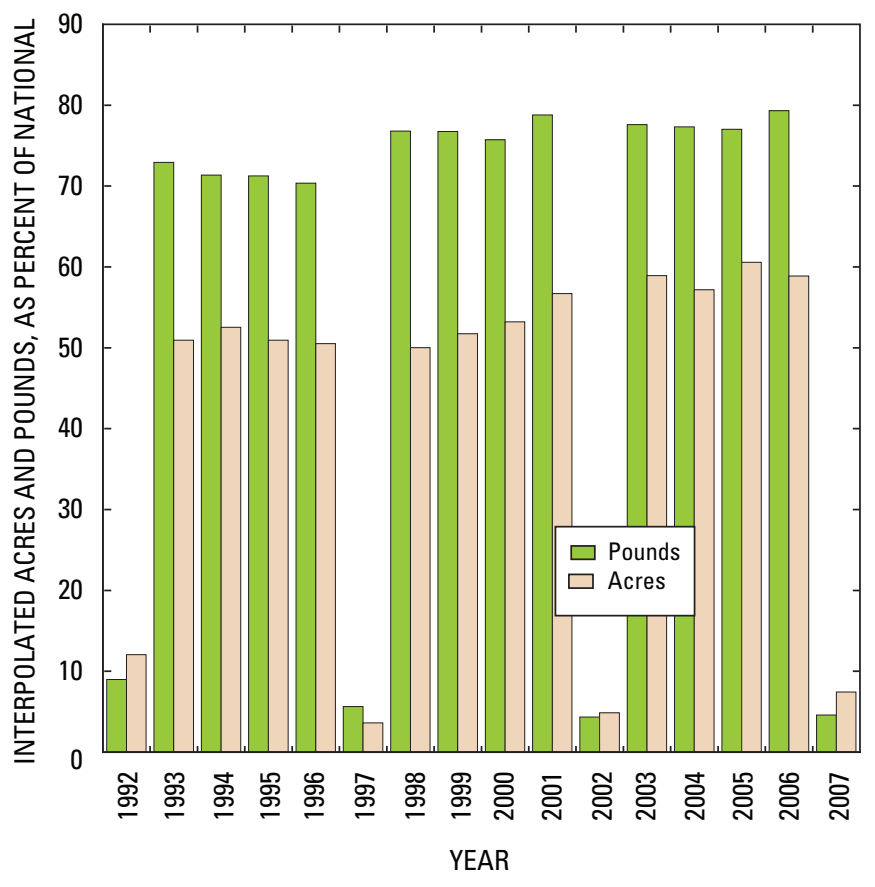

Figure 8. Percentage of atrazine use and acreage estimates derived from interpolated crop acreage, 1992-2007. because NASS reports only sorghum for grain acreage, in nonCensus years acreage for these subcategories was based on interpolation of Census acres and added to the NASS sorghum acres.

The acreage interpolation method added a much larger portion of the acres for non-Census years than for the Census years (fig. 8). To determine if there was a systematic difference between the Census and NASS, 1992, 1997, 2002, and 2007 county harvested acreage estimates from these sources were compared (fig. 9). Corn for grain was selected for this comparison because about 70 percent of all atrazine is applied to corn acreage and if corn acreage is significantly under or over estimated for a given year, the atrazine application rates for corn will be affected as will the atrazine estimate for the counties. The graphs in figure 9 indicate that county estimates of harvested corn acreage from the two USDA sources are about the same, although there is somewhat more variability in 2007 estimates, particularly for some counties with a large amount of corn acreage. Corn acreage in about 300 counties reported in the Census was not reported by NASS, and conversely, NASS reported acreage for about a dozen counties not reported by the Census. County acreage of corn for grain was not reported by NASS in 1997 or 2002 for Connecticut, Maine, Massachusetts, New Hampshire, and Vermont; the crop acreage in these states from Census years was interpolated to fill in acreage for the counties not reported by NASS. 

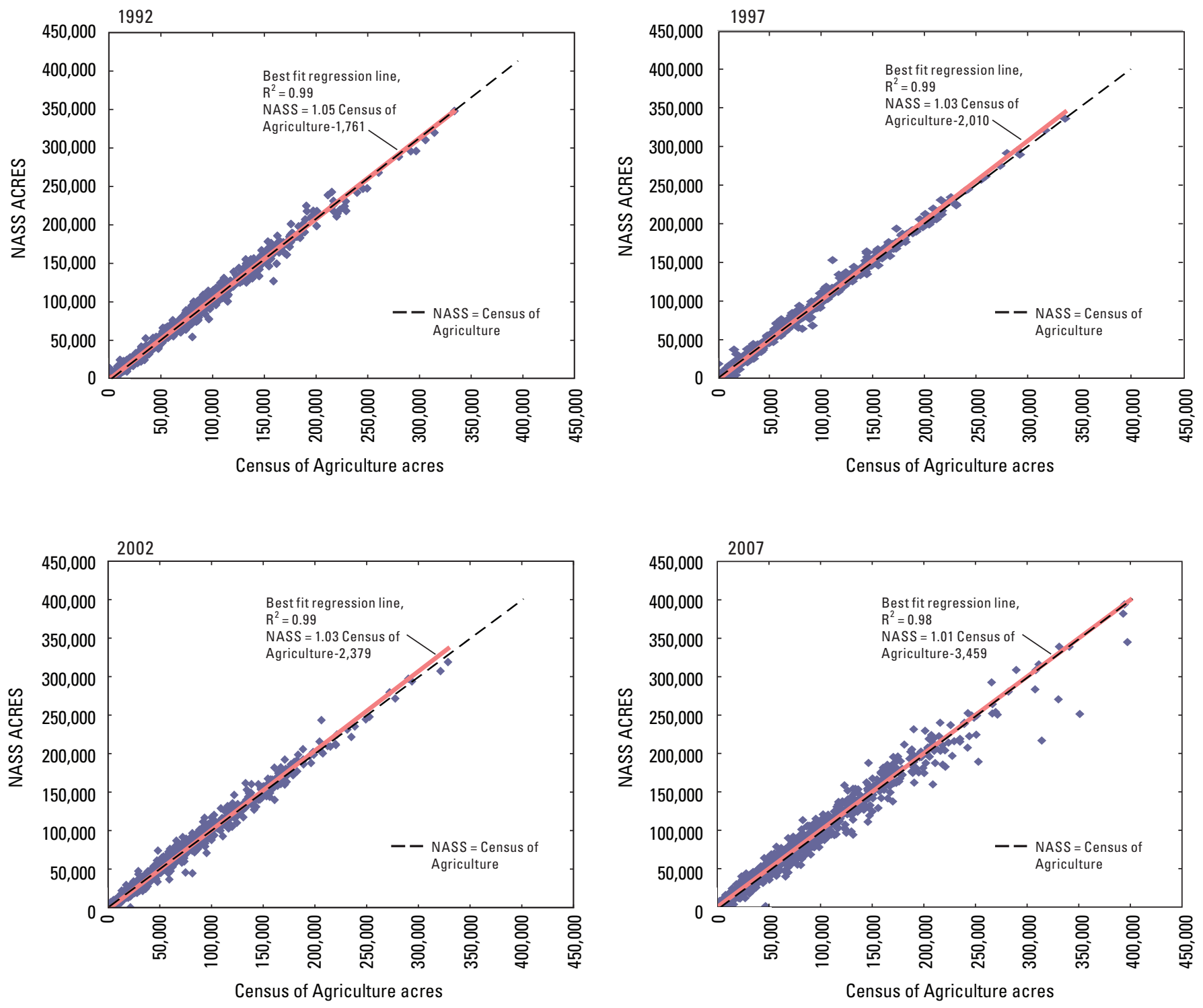

Figure 9. Comparison of Census of Agriculture and National Agriculture Statistics Service (NASS) estimates of corn acreage in a county for 1992, 1997, 2002, and 2007. 


\section{Comparison of EPest-Use Estimates with Estimates from Other Sources}

Atrazine-use estimates produced by the method described in this report were compared with data from other available sources in order to evaluate the reasonableness of the EPest-use estimates. Data were collected from: (1) USDA's Agricultural Resource Management Survey (ARMS) and published in the NASS Chemical Use Database at (http:// www.pestmanagement.info/nass/app usage.cfm), (2) countylevel estimates developed by Thelin and Gianessi (2000) using NPUD state data, and (3) USEPA estimates for atrazine use in the agricultural market sector (Kiely and others, 2004).

Since 1991, ARMS has collected information on agricultural practices, including chemical and fertilizer use, through annual statistical surveys of major field crops and fruit, nut, and vegetable crops. NASS does not survey the same states or crops every year, but instead rotates crops and states in and out of the survey on the basis of a number of criteria, including the proportion of national acreage the state represents and the geography represented. The survey is based on a statistical sample of all farm and ranch operations in the conterminous 48 states that have an annual income of $\$ 1,000$ or more and is conducted by personal interview. The resulting data are publicly available and are an important benchmark to compare against. Hereinafter, data from the USDA Chemical Use Database will be referred to as NASS-CU.

NPUD atrazine estimates were produced by applying NPUD state chemical application rates to county harvested crop acreage and adding the county estimates for each conterminous state and then summing the state totals to produce a total for the conterminous United States. USEPA estimates of atrazine use were compiled from a variety of public and proprietary information sources and are reported as low to high ranges of pounds used in the agricultural sector.

\section{Comparison of National Use Estimates}

Annual national atrazine-use estimates for agriculture from the EPest county database, AgroTrak, NPUD, and USEPA are compared in figure 10. National totals compared were derived for EPest and NPUD by summing the county estimates, NASS-CU annual estimates were published as the total pounds per year for 'All Program States'; and the USEPA estimates were reported as a low to high range of atrazine pounds. EPest national totals were higher than NPUD totals by about 14 percent in 1992, 4 percent in 1997, and 3 percent in 2002. EPest totals and USEPA estimates were compared for 11 years and EPest estimates were about in the middle of the low and high range of atrazine estimates reported by USEPA except in 2003, when the EPest total was about 3 percent higher than the USEPA high estimate. The NASS-CU national totals represent only the amount of atrazine used on the crops and states surveyed in a specific year, so these totals cannot be compared to the total atrazine use estimated by other methods.
Because of the NASS rotational survey method, the NASS-CU values for each year are not intended to be national totals and it is expected that the NASS-CU total would be lower than the other sources compared. In addition, atrazine estimates could not be compared for every year because, at the time of this report, NASS-CU national estimates were not available for 2005 and 2007.

\section{Comparison of State Use Estimates}

EPest estimates were also compared with state-level estimates from NASS-CU and NPUD. In addition to showing variability of estimates at this smaller scale, this approach allowed direct comparison with NASS-CU estimates for specific states and years surveyed. EPest state estimates for atrazine use on corn were compared with state estimates from (1) NASS-CU and NPUD data for Census years 1992, 1997, and 2007; and (2) NASS-CU data for non-Census years. This comparison focused on atrazine use on corn because of the

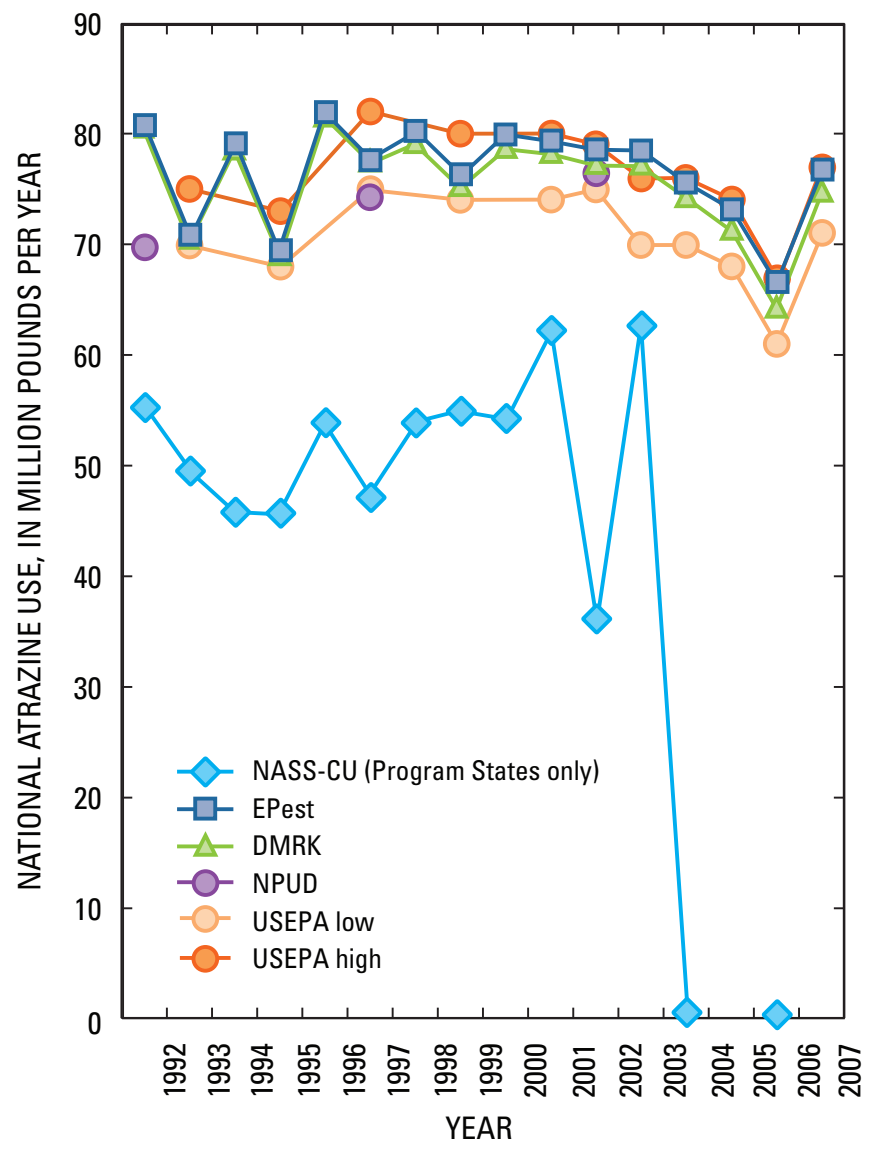

Figure 10. National atrazine-use estimates for agricultural purposes, 1992-2007. Data from EPest, Doane Marketing Research-Kynetec (DMRK), National Agriculture Statistics Service Chemical Use (NASS-CU), National Pesticide Use Database (NPUD), and U.S. Environmental Protection Agency (USEPA). 
limited availability of NASS-CU data for atrazine use in all states and on all the crops surveyed by DMRK and included in the county database. EPest and NPUD annual state totals were derived by summing county-level atrazine estimates for corn to the state-level and NASS-CU state totals that were retrieved from http://www.pestmanagement.info/nass/app-usage.cfm. The state comparisons for Census years were based on NPUD data for 48 states in 1992 and 1997 and 47 states in 2002 and on published NASS-CU state data for 17 states in 1992, 10 states in 1997, and 7 states in 2002.

The state-by-state comparisons for Census years show good agreement between EPest estimates and both NPUD (fig. 11A) and NASS (fig. 11B), although EPest state totals tend to be slightly greater. The NPUD and EPest estimates were highly correlated $\left(\mathrm{R}^{2}=0.99\right)$ with a slope close to $1(\mathrm{y}=0.97 \mathrm{x}-45,829)$. NASS-CU and EPest estimates had a similar correlation $\left(\mathrm{R}^{2}=0.98\right)$, but a lower slope $(\mathrm{y}=0.89 \mathrm{x}$ $+42,377)$, indicating the tendency of EPest estimates to be higher than NASS-CU estimates for most states. There were some states, such as Nevada, New Hampshire, and Montana, for which NPUD estimates were higher for corn than EPest estimates, and in many of these cases, EPest estimates for these states were based on unsurveyed rates. NASS-CU and EPest state comparisons for Census years also showed that EPest atrazine totals tended to be greater than NASS-CU totals for most of the years compared, including estimates for major corn producing states, such as Indiana and Iowa, which differed by about 15 percent. However, three states (Michigan, North Dakota, and South Dakota) had NASS-CU atrazine estimates that were 10 percent or more greater than the EPest estimate. In non-Census years, EPest and NASS-CU estimates also had good agreement $\left(\mathrm{R}^{2}=0.95\right)$; these comparisons were based on as few as 3 states in 2000 and as many as 19 states in 2001 and 2005 (not shown).

Differences in geographic and temporal coverage and methods used to collect pesticide use information are factors that contribute to the variation in atrazine estimates for corn from the three datasets compared. Both NASS and EPest data are based on statistical surveys which are selective by their design and dependent on sampling density to produce statistically reliable estimates for states and the nation. EPest and NASS-CU data are highly correlated when compared state-by-state for the same years; however, because of the constraints of NASS sample design, atrazine use on corn could not be compared for all states that had an EPest estimate. Although NPUD data are derived from NASS survey data for multiple years, NPUD also incorporates information from chemical use specialists and when necessary, uses application rates imputed from neighboring states to produce a more complete set of chemical application rates for the conterminous United States.
A.

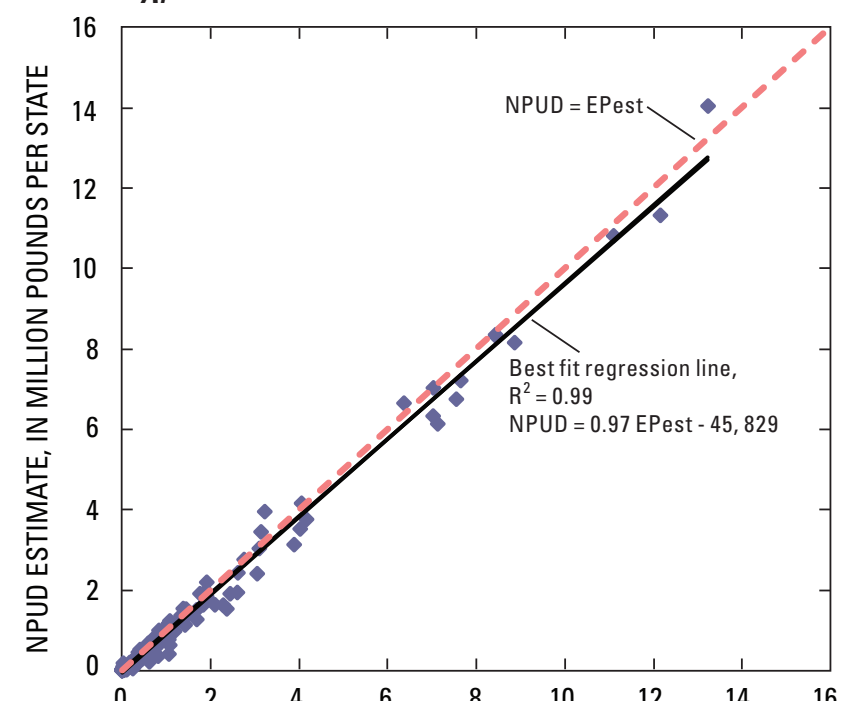

B.

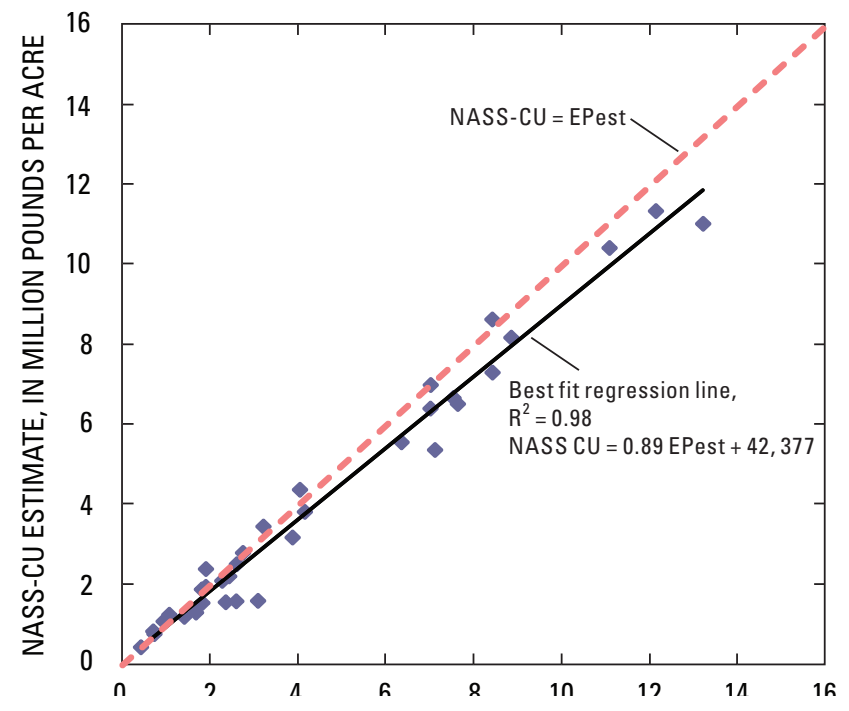

Figure 11. Comparison of EPest and $(A)$ National Pesticide Use Database (NPUD) and $(B)$ National Agriculture Statistics Service Chemical Use (NASS-CU) estimates of state atrazine use on corn. 


\section{Application of County Atrazine-Use Data}

For U.S. Geological Survey water-quality studies, a primary application of atrazine-use estimates produced in this study is as an explanatory variable in regression models developed to predict atrazine concentrations in streams by using watershed characteristics. The Watershed Regressions for Pesticides (WARP) model developed using watershed atrazine-use estimates from NPUD data (Larson and others, 2004) indicated that pesticide use in a watershed was the most influential variable for predicting atrazine concentrations in a stream. An updated WARP model (Stone, and Gilliom, 2009) was developed that incorporates annual concentration statistics, and annual use estimates for watersheds using EPest 1992-1999 county-level atrazine data.

Annual atrazine use was estimated from EPest county atrazine-use data for 112 watershed between 1992 through 1999 and used as input to the updated WARP model following the method described by Nakagaki and Wolock (2005). This method included the development of a basin land-covercounty weighting factor that was produced through a series of GIS processes in which GIS coverages of county boundaries and basin boundaries were intersected with an enhanced version of the 1992 National Land Cover Dataset (NLCD 1992) (Nakagaki and others, 2007) in order to determine: (1) the counties that were entirely or partially contained in each of the 112 watersheds, and (2) the percentage of three types of NLCD agricultural land cover (row crops/grains/ fallow, orchards/vineyards, and pasture/hay) within each of the counties in the watershed. The weighting factors were used to allocate county-level pesticide use by crop to the three landcover classes. The watershed use-intensity estimate, expressed in kilograms of atrazine applied to watershed area $\left(\mathrm{km}^{2}\right)$, was calculated by summing the pesticide use estimates for the counties entirely and partially contained in the watershed and dividing the total mass by the watershed area. Atrazine use-intensity estimates for the 112 watersheds used in WARP model development were calculated using EPest 1992 through 1999 atrazine-use estimates and 1992 NLCD land-cover data and are included in appendix table A-1.

As described above, land-cover information is a key component of the method used to estimate agricultural atrazine use in a watershed. Although this step in the process does not affect estimated use contributions from counties that are entirely within a watershed, this information is used to allocate a portion of a county's use to a watershed for any county that is not entirely in the watershed. To date, the 1992 version of NLCD agricultural land-cover data has been used to allocate NPUD and EPest county-level pesticide-use estimates to a watershed. An updated version of the NLCD dataset has been developed by using data centered on the year 2001; it provides updated information on the spatial distribution and area of various land-cover classes across the conterminous United States. Although the 2001 NLCD (Homer and others, 2004) data were produced using data sources and methods similar to the 1992 NLCD data, two slightly different classification systems were used to map land-cover. The 1992 NLCD dataset identifies five agricultural land-cover classes (pasture/ hay, row crops, small grains, fallow, and orchards and vineyards), whereas the 2001 NLCD dataset identifies two broad agricultural classes (pasture/hay and cultivated crops that includes orchards and vineyards). A cross-walk (table 3 ) between the specific crops contained in the EPest countyatrazine database and the 1992 and 2001 NLCD agricultural land-cover classes was used to select and sum atrazine use for specific crops and associate them with the appropriate 1992 or 2001 agricultural land-cover class.

The 1992 and 2001 NLCD datasets provide information on the amount and spatial distribution of land-cover across almost a decade, and provide a framework to which the EPest multi-year atrazine-use data can be linked and to improve the characterization of agricultural pesticide use over time. For studies evaluating pesticide use trends that span the range of years for 1992 through 2007, it may be appropriate to (1) generalize the 1992 NLCD land-cover data to make it comparable with the 2001 NLCD by combining orchards and vineyards, small grains and fallow with the row crop category and (2) associate 1992-1997 EPest county pesticide use data with the general 1992 NLCD land-cover data and 1998-2007 EPest county pesticide use with the 2001 NLCD data. This break-point was selected because beginning in 1998 AgroTrak data collection methods changed and the 2001 NLCD data includes some data collected in the late 1990s. However, for studies where improved land-cover information is available, these break points may not be appropriate.

\section{Summary and Conclusions}

A method was developed to estimate annual atrazine use on agricultural crops for all counties in the conterminous United States from 1992 to 2007. For all conterminous states, except California, atrazine crop application rates were developed on the basis of atrazine-use estimates for Crop Reporting Districts (CRD) included in the proprietary AgroTrak database developed by Doane Marketing ResearchKynetec (DMRK), and from publicly available data for annual county harvested crop acreage reported by the U.S. Department of Agriculture. To calculate atrazine cropapplication rates for the CRDs surveyed by DMRK, harvested acreage rather than planted acreage was used because the USDA reports county harvested acreage for almost all agricultural crops, whereas planted acreage is reported for only a subset of these crops. The atrazine crop-application rate was developed by dividing the DMRK-AgroTrak estimate of the mass of atrazine applied to a crop in the CRD by the harvested acreage for that crop in the CRD. The DMRK-AgroTrak 
database included atrazine-use data for most CRDs in the conterminous United States for most years between 1992 and 2007. If atrazine-use data were not available for a CRD and crop for which USDA reported harvested acreage, an atrazine crop application rate (EPest rate) for that crop and year was estimated on the basis of rates determined through proximity analysis of surrounding CRDs. A hierarchy of decision rules was followed to develop a rate for the crop and year for the CRD without a rate. These rules included (1) calculating a Tier 1 rate on the basis of the median rates of the DMRK surveyed rate(s) for the CRDs adjacent to the primary CRD or (2) if a Tier 1 rate was not available, calculating a Tier 2 rate using the median rate derived from a minimum of three DMRK surveyed CRDs adjacent to the Tier 1 CRDs, or (3) calculating the median of DMRK surveyed rates, Tier 1, or Tier 2 EPest rates from the CRDs that were part of the same USDA Farm Production Region, and lastly, (4) calculating a median crop application rate using available surveyed CRD rates from DMRK, and, or Tier 1, and Tier 2 CRDS that are part of the same USDA Farm Production Region for any year(s) between 1992 and 2007. CRD crop application rates were then applied to county harvested-acreage values to estimate county atrazine use by crop, except in California, where county use was estimated directly from pesticide applicators usage reports.

Total atrazine use derived from unsurveyed Tier 1 , Tier 2, or regional EPest rates contributed 2 percent or less to the national atrazine total estimates in any year; however, the method described above did not cover crops for years when harvested acres for a crop and county were not reported in the Census of Agriculture or NASS. Atrazine use in unsurveyed CRDS was important for some crops, particularly outside of the primary production areas of a crop and consequently where there were fewer DMRK-AgroTrak surveys.

To ensure that all crop acres were included in the development of crop application rates and that atrazine use on all crop acres in a county for all years was accounted for, the method developed in this study used linear interpolation to estimate acreage, for a crop that was not reported, by using data on acreage that was reported for another year. Crop acreage added by the interpolation method increased the estimated national acreage for all crops by less than 20 percent. The acreage interpolation method was used most often to estimate county crop acreage in non-Census years for minor crops not reported by NASS, and less often to estimate acreage for major crops in years when NASS did not conduct a crop survey in a state.

EPest estimates of annual total atrazine use for the conterminous United States were compared with published data from USEPA, NPUD and NASS-CU. EPest totals agreed well with NPUD and USEPA estimates of total agricultural atrazine use, and were consistently higher than estimates reported by NASS, between 1992 and 2004 for selected crops and states. State-level atrazine use on corn was limited to comparisons between EPest and NPUD and EPest and NASS-CU. Atrazine-use estimates from EPest and NASS were compared for as few as seven states and as many as 48 states when comparing EPest and NPUD data. EPest, NPUD, and NASS estimates were highly correlated, although EPest estimates tended to be slightly higher than those from NASS.

This study used the herbicide atrazine for method development and testing because of the availability of extensive temporal and geographic data on atrazine use between 1992 and 2007. Tier 1, Tier 2, and regional EPest rates for unsurveyed CRDs enable estimation of atrazine use for all counties that reported harvested acreage of the crops on which atrazine was applied. Applying Tier 1, Tier 2 and regional EPest rates to counties in unsurveyed CRDs ensures that atrazine use is accounted for in areas of the country that have few DMRK-AgroTrak surveys. The method developed in this study should be applicable to pesticides included in DMRK-AgroTrak that have widespread use and relatively consistent usage patterns. However, the method will most likely need to be adjusted for insecticides and fungicides that do not have consistent geographic and temporal usage patterns and for pesticides that are primarily applied to specialty crops. In these cases, pesticide use estimated on the basis of Tier 1, Tier 2 or regional rates and interpolated crop acreage may represent a larger proportion of the estimated use, resulting in greater uncertainty in use estimates.

\section{Acknowledgments}

The authors would like to thank Hylon Kauffman, Cliff Schasch, and John Thompson of DMRKynetec for their technical support and useful discussions that have provided a better understanding of the data, collection methods, and extrapolation procedures used in the development of AgroTrak. The authors would also like to acknowledge the GIS expertise of Naomi Nakagaki of the USGS, who developed the table of CRD relationships used to develop application rates for non-surveyed CRDs. The authors thank Bill Battaglin and Nancy Baker for their valuable comments during the USGS review process.

\section{References Cited}

California Department of Pesticide Regulation, 2009, California Pesticide Information Portal (CALPIP), accessed on July 8, 2009, at http://www.cdpr.ca.gov/docs/pur/ purmain.htm

Gianessi, L.P., and Anderson, J.E., 1995, Pesticide use in U.S. crop production - National summary report: Washington, D.C., National Center for Food and Agricultural Policy, 280 p. 
Gianessi, L.P., and Marcelli M.B., 2000, Pesticide use in U.S. crop production: 1997-National summary report: Washington, D.C., National Center for Food and Agricultural Policy, variously paged. Available at http:// www.croplifefoundation.org/Documents/PUD/1997\%20 PUD\%20National\%20Summary.pdf

Gianessi, L.P., and Reigner, N., 2006, Pesticide use in U.S. crop production: 2002 - Insecticides \& other pesticides: Washington, D.C., CropLife Foundation, 34 p. Available at http://www.croplifefoundation.org/Documents/PUD/ NPUD $\% 202002 /$ Inst $\% 20$ and $\% 200 P \% 20$ Data $\% 20$ Report. pdf

Homer, Collin, Huang, Chengquan, Yang, Limin, Wylie, Bruce, and Coan, Michael, 2004, Development of a 2001 national land-cover database for the United States: Photogrammetric Engineering and Remote Sensing, v. 70, no. 7, p. 829-840. Available at http://www.mrlc.gov/pdf/ July_PERS.pdf

Kiely, T., Donaldson, D., and Grube, A., 2004, Pesticides industry sales and usage-2000 and 2001 market estimates: U.S. Environmental Protection Agency, Office of Prevention, Pesticides, and Toxic Substances, EPA-733-R 04-001, $33 \mathrm{p}$.

Larson, S.J., Crawford, C.G., and Gilliom, R.J., 2004, Development and application of watershed regressions for pesticides (WARP) for estimating atrazine concentration distributions in streams: U.S. Geological Survey WaterResource Investigations Report 3-4047, 68 p.

Nakagaki, Naomi, Price, C.P., Falcone, J.A., Hitt, K.J., and Ruddy, B.C., 2007, Enhanced national land cover data 1992 (NLCDe 92), version 1.0, [digital map], accessed December 1, 2007, at http://water.usgs.gov/lookup/getspatial?nlcde92
Nakagaki, Naomi, and Wolock, D.M., 2005, Estimation of agricultural pesticide use in drainage basins using land cover maps and county pesticide data: U.S. Geological Survey Open File Report 2005-1188, 46 p.

Stone, W.W., and Gilliom, R.J., 2009, Update of watershed regressions for pesticides (WARP) for predicting atrazine concentration in streams: U.S. Geological Survey Open-File Report 2009-1122, 22 p.

Thelin, G.P., and Gianessi, L.P., 2000, Method for estimating pesticide use for county areas of the United States: U.S. Geological Survey Open-File Report 2000-25, 62 p.

U.S. Department of Agriculture, 1998, Agriculture fact book 1998, accessed December 18, 2008, at http://www.usda.gov/ news/pubs/fbook98/content.htm

U.S. Department of Agriculture, 2009, Census of Agriculture, accessed January 7, 2009 at http://www.agcensus.usda.gov/

U.S. Department of Agriculture, 2009, National Agricultural Statistics Service (NASS). Agricultural chemical use database, accessed January 7, 2009, at http://www. pestmanagement.info/nass/

U.S. Department of Agriculture, 2009, National Agricultural Statistics Service (NASS). Quick Stats, U.S. \& All States County Data- Crops., accessed January 7, 2009 at http:// www.nass.usda.gov/Data_and_Statistics/Quick_Stats/index. $\underline{\text { asp }}$

U.S. Environmental Protection Agency, 2009, Pesticide Product Label System (PPLS), accessed on July 8, 2009, at http://www.epa.gov/pesticides/pestlabels/ 


\section{Tables}


Table 1. Crop Reporting Districts (CRD) of the conterminous United States and associated counties.

[FIPS, Federal Information Processing Standard; USDA, U.S. Department of Agriculture]

\begin{tabular}{|c|c|c|c|c|c|}
\hline State & $\begin{array}{l}\text { Crop Reporting District } \\
\text { (CRD) }\end{array}$ & CRD Identifier & County Name & County FIPS & $\begin{array}{c}\text { USDA Farm Production } \\
\text { Region }\end{array}$ \\
\hline Alabama & Alabama CRD 40 & 1040 & Autauga & 1001 & Southeast \\
\hline Alabama & Alabama CRD 50 & 1050 & Baldwin & 1003 & Southeast \\
\hline Alabama & Alabama CRD 60 & 1060 & Barbour & 1005 & Southeast \\
\hline Alabama & Alabama CRD 30 & 1030 & Bibb & 1007 & Southeast \\
\hline Alabama & Alabama CRD 20 & 1020 & Blount & 1009 & Southeast \\
\hline Alabama & Alabama CRD 40 & 1040 & Bullock & 1011 & Southeast \\
\hline Alabama & Alabama CRD 50 & 1050 & Butler & 1013 & Southeast \\
\hline Alabama & Alabama CRD 20 & 1020 & Calhoun & 1015 & Southeast \\
\hline Alabama & Alabama CRD 30 & 1030 & Chambers & 1017 & Southeast \\
\hline Alabama & Alabama CRD 20 & 1020 & Cherokee & 1019 & Southeast \\
\hline Alabama & Alabama CRD 30 & 1030 & Chilton & 1021 & Southeast \\
\hline Alabama & Alabama CRD 50 & 1050 & Choctaw & 1023 & Southeast \\
\hline Alabama & Alabama CRD 50 & 1050 & Clarke & 1025 & Southeast \\
\hline Alabama & Alabama CRD 30 & 1030 & Clay & 1027 & Southeast \\
\hline Alabama & Alabama CRD 20 & 1020 & Cleburne & 1029 & Southeast \\
\hline Alabama & Alabama CRD 60 & 1060 & Coffee & 1031 & Southeast \\
\hline Alabama & Alabama CRD 10 & 1010 & Colbert & 1033 & Southeast \\
\hline Alabama & Alabama CRD 50 & 1050 & Conecuh & 1035 & Southeast \\
\hline Alabama & Alabama CRD 30 & 1030 & Coosa & 1037 & Southeast \\
\hline Alabama & Alabama CRD 60 & 1060 & Covington & 1039 & Southeast \\
\hline Alabama & Alabama CRD 60 & 1060 & Crenshaw & 1041 & Southeast \\
\hline Alabama & Alabama CRD 20 & 1020 & Cullman & 1043 & Southeast \\
\hline Alabama & Alabama CRD 60 & 1060 & Dale & 1045 & Southeast \\
\hline Alabama & Alabama CRD 40 & 1040 & Dallas & 1047 & Southeast \\
\hline Alabama & Alabama CRD 20 & 1020 & De Kalb & 1049 & Southeast \\
\hline Alabama & Alabama CRD 40 & 1040 & Elmore & 1051 & Southeast \\
\hline Alabama & Alabama CRD 50 & 1050 & Escambia & 1053 & Southeast \\
\hline Alabama & Alabama CRD 20 & 1020 & Etowah & 1055 & Southeast \\
\hline Alabama & Alabama CRD 30 & 1030 & Fayette & 1057 & Southeast \\
\hline Alabama & Alabama CRD 10 & 1010 & Franklin & 1059 & Southeast \\
\hline Alabama & Alabama CRD 60 & 1060 & Geneva & 1061 & Southeast \\
\hline Alabama & Alabama CRD 40 & 1040 & Greene & 1063 & Southeast \\
\hline Alabama & Alabama CRD 40 & 1040 & Hale & 1065 & Southeast \\
\hline Alabama & Alabama CRD 60 & 1060 & Henry & 1067 & Southeast \\
\hline Alabama & Alabama CRD 60 & 1060 & Houston & 1069 & Southeast \\
\hline Alabama & Alabama CRD 20 & 1020 & Jackson & 1071 & Southeast \\
\hline Alabama & Alabama CRD 30 & 1030 & Jefferson & 1073 & Southeast \\
\hline Alabama & Alabama CRD 30 & 1030 & Lamar & 1075 & Southeast \\
\hline Alabama & Alabama CRD 10 & 1010 & Lauderdale & 1077 & Southeast \\
\hline Alabama & Alabama CRD 10 & 1010 & Lawrence & 1079 & Southeast \\
\hline Alabama & Alabama CRD 30 & 1030 & Lee & 1081 & Southeast \\
\hline Alabama & Alabama CRD 10 & 1010 & Limestone & 1083 & Southeast \\
\hline
\end{tabular}


Table 1. Crop Reporting Districts (CRD) of the conterminous United States and associated counties. - Continued

[FIPS, Federal Information Processing Standard; USDA, U.S. Department of Agriculture]

\begin{tabular}{|c|c|c|c|c|c|}
\hline State & $\begin{array}{l}\text { Crop Reporting District } \\
\text { (CRD) }\end{array}$ & CRD Identifier & County Name & County FIPS & $\begin{array}{l}\text { USDA Farm Production } \\
\text { Region }\end{array}$ \\
\hline Alabama & Alabama CRD 40 & 1040 & Lowndes & 1085 & Southeast \\
\hline Alabama & Alabama CRD 40 & 1040 & Macon & 1087 & Southeast \\
\hline Alabama & Alabama CRD 10 & 1010 & Madison & 1089 & Southeast \\
\hline Alabama & Alabama CRD 40 & 1040 & Marengo & 1091 & Southeast \\
\hline Alabama & Alabama CRD 10 & 1010 & Marion & 1093 & Southeast \\
\hline Alabama & Alabama CRD 20 & 1020 & Marshall & 1095 & Southeast \\
\hline Alabama & Alabama CRD 50 & 1050 & Mobile & 1097 & Southeast \\
\hline Alabama & Alabama CRD 50 & 1050 & Monroe & 1099 & Southeast \\
\hline Alabama & Alabama CRD 40 & 1040 & Montgomery & 1101 & Southeast \\
\hline Alabama & Alabama CRD 10 & 1010 & Morgan & 1103 & Southeast \\
\hline Alabama & Alabama CRD 40 & 1040 & Perry & 1105 & Southeast \\
\hline Alabama & Alabama CRD 30 & 1030 & Pickens & 1107 & Southeast \\
\hline Alabama & Alabama CRD 60 & 1060 & Pike & 1109 & Southeast \\
\hline Alabama & Alabama CRD 30 & 1030 & Randolph & 1111 & Southeast \\
\hline Alabama & Alabama CRD 60 & 1060 & Russell & 1113 & Southeast \\
\hline Alabama & Alabama CRD 20 & 1020 & St. Clair & 1115 & Southeast \\
\hline Alabama & Alabama CRD 30 & 1030 & Shelby & 1117 & Southeast \\
\hline Alabama & Alabama CRD 40 & 1040 & Sumter & 1119 & Southeast \\
\hline Alabama & Alabama CRD 30 & 1030 & Talladega & 1121 & Southeast \\
\hline Alabama & Alabama CRD 30 & 1030 & Tallapoosa & 1123 & Southeast \\
\hline Alabama & Alabama CRD 30 & 1030 & Tuscaloosa & 1125 & Southeast \\
\hline Alabama & Alabama CRD 30 & 1030 & Walker & 1127 & Southeast \\
\hline Alabama & Alabama CRD 50 & 1050 & Washington & 1129 & Southeast \\
\hline Alabama & Alabama CRD 50 & 1050 & Wilcox & 1131 & Southeast \\
\hline Alabama & Alabama CRD 10 & 1010 & Winston & 1133 & Southeast \\
\hline Arizona & Arizona CRD 10 & 4010 & Yavapai & 4025 & Mountain \\
\hline Arizona & Arizona CRD 10 & 4010 & Mohave & 4015 & Mountain \\
\hline Arizona & Arizona CRD 10 & 4010 & Navajo & 4017 & Mountain \\
\hline Arizona & Arizona CRD 10 & 4010 & Gila & 4007 & Mountain \\
\hline Arizona & Arizona CRD 10 & 4010 & Apache & 4001 & Mountain \\
\hline Arizona & Arizona CRD 10 & 4010 & Coconino & 4005 & Mountain \\
\hline Arizona & Arizona CRD 80 & 4080 & Yuma & 4027 & Mountain \\
\hline Arizona & Arizona CRD 80 & 4080 & Pima & 4019 & Mountain \\
\hline Arizona & Arizona CRD 80 & 4080 & Santa Cruz & 4023 & Mountain \\
\hline Arizona & Arizona CRD 80 & 4080 & Pinal & 4021 & Mountain \\
\hline Arizona & Arizona CRD 80 & 4080 & Graham & 4009 & Mountain \\
\hline Arizona & Arizona CRD 80 & 4080 & Cochise & 4003 & Mountain \\
\hline Arizona & Arizona CRD 80 & 4080 & Greenlee & 4011 & Mountain \\
\hline Arizona & Arizona CRD 80 & 4080 & Maricopa & 4013 & Mountain \\
\hline Arizona & Arizona CRD 80 & 4080 & $\mathrm{La} \mathrm{Paz}$ & 4012 & Mountain \\
\hline Arkansas & Arkansas CRD 60 & 5060 & Arkansas & 5001 & Delta \\
\hline Arkansas & Arkansas CRD 90 & 5090 & Ashley & 5003 & Delta \\
\hline
\end{tabular}


Table 1. Crop Reporting Districts (CRD) of the conterminous United States and associated counties.-Continued

[FIPS, Federal Information Processing Standard; USDA, U.S. Department of Agriculture]

\begin{tabular}{|c|c|c|c|c|c|}
\hline State & $\begin{array}{l}\text { Crop Reporting District } \\
\text { (CRD) }\end{array}$ & CRD Identifier & County Name & County FIPS & $\begin{array}{c}\text { USDA Farm Production } \\
\text { Region }\end{array}$ \\
\hline Arkansas & Arkansas CRD 20 & 5020 & Baxter & 5005 & Delta \\
\hline Arkansas & Arkansas CRD 10 & 5010 & Benton & 5007 & Delta \\
\hline Arkansas & Arkansas CRD 10 & 5010 & Boone & 5009 & Delta \\
\hline Arkansas & Arkansas CRD 80 & 5080 & Bradley & 5011 & Delta \\
\hline Arkansas & Arkansas CRD 80 & 5080 & Calhoun & 5013 & Delta \\
\hline Arkansas & Arkansas CRD 10 & 5010 & Carroll & 5015 & Delta \\
\hline Arkansas & Arkansas CRD 90 & 5090 & Chicot & 5017 & Delta \\
\hline Arkansas & Arkansas CRD 80 & 5080 & Clark & 5019 & Delta \\
\hline Arkansas & Arkansas CRD 30 & 5030 & Clay & 5021 & Delta \\
\hline Arkansas & Arkansas CRD 20 & 5020 & Cleburne & 5023 & Delta \\
\hline Arkansas & Arkansas CRD 80 & 5080 & Cleveland & 5025 & Delta \\
\hline Arkansas & Arkansas CRD 80 & 5080 & Columbia & 5027 & Delta \\
\hline Arkansas & Arkansas CRD 50 & 5050 & Conway & 5029 & Delta \\
\hline Arkansas & Arkansas CRD 30 & 5030 & Craighead & 5031 & Delta \\
\hline Arkansas & Arkansas CRD 40 & 5040 & Crawford & 5033 & Delta \\
\hline Arkansas & Arkansas CRD 60 & 5060 & Crittenden & 5035 & Delta \\
\hline Arkansas & Arkansas CRD 60 & 5060 & Cross & 5037 & Delta \\
\hline Arkansas & Arkansas CRD 80 & 5080 & Dallas & 5039 & Delta \\
\hline Arkansas & Arkansas CRD 90 & 5090 & Desha & 5041 & Delta \\
\hline Arkansas & Arkansas CRD 90 & 5090 & Drew & 5043 & Delta \\
\hline Arkansas & Arkansas CRD 50 & 5050 & Faulkner & 5045 & Delta \\
\hline Arkansas & Arkansas CRD 40 & 5040 & Franklin & 5047 & Delta \\
\hline Arkansas & Arkansas CRD 20 & 5020 & Fulton & 5049 & Delta \\
\hline Arkansas & Arkansas CRD 50 & 5050 & Garland & 5051 & Delta \\
\hline Arkansas & Arkansas CRD 50 & 5050 & Grant & 5053 & Delta \\
\hline Arkansas & Arkansas CRD 30 & 5030 & Greene & 5055 & Delta \\
\hline Arkansas & Arkansas CRD 70 & 5070 & Hempstead & 5057 & Delta \\
\hline Arkansas & Arkansas CRD 50 & 5050 & Hot Spring & 5059 & Delta \\
\hline Arkansas & Arkansas CRD 70 & 5070 & Howard & 5061 & Delta \\
\hline Arkansas & Arkansas CRD 30 & 5030 & Independence & 5063 & Delta \\
\hline Arkansas & Arkansas CRD 20 & 5020 & Izard & 5065 & Delta \\
\hline Arkansas & Arkansas CRD 30 & 5030 & Jackson & 5067 & Delta \\
\hline Arkansas & Arkansas CRD 90 & 5090 & Jefferson & 5069 & Delta \\
\hline Arkansas & Arkansas CRD 40 & 5040 & Johnson & 5071 & Delta \\
\hline Arkansas & Arkansas CRD 70 & 5070 & Lafayette & 5073 & Delta \\
\hline Arkansas & Arkansas CRD 30 & 5030 & Lawrence & 5075 & Delta \\
\hline Arkansas & Arkansas CRD 60 & 5060 & Lee & 5077 & Delta \\
\hline Arkansas & Arkansas CRD 90 & 5090 & Lincoln & 5079 & Delta \\
\hline Arkansas & Arkansas CRD 70 & 5070 & Little River & 5081 & Delta \\
\hline Arkansas & Arkansas CRD 40 & 5040 & Logan & 5083 & Delta \\
\hline Arkansas & Arkansas CRD 60 & 5060 & Lonoke & 5085 & Delta \\
\hline Arkansas & Arkansas CRD 10 & 5010 & Madison & 5087 & Delta \\
\hline
\end{tabular}


Table 1. Crop Reporting Districts (CRD) of the conterminous United States and associated counties. - Continued

[FIPS, Federal Information Processing Standard; USDA, U.S. Department of Agriculture]

\begin{tabular}{|c|c|c|c|c|c|}
\hline State & $\begin{array}{l}\text { Crop Reporting District } \\
\text { (CRD) }\end{array}$ & CRD Identifier & County Name & County FIPS & $\begin{array}{c}\text { USDA Farm Production } \\
\text { Region }\end{array}$ \\
\hline Arkansas & Arkansas CRD 20 & 5020 & Marion & 5089 & Delta \\
\hline Arkansas & Arkansas CRD 70 & 5070 & Miller & 5091 & Delta \\
\hline Arkansas & Arkansas CRD 30 & 5030 & Mississippi & 5093 & Delta \\
\hline Arkansas & Arkansas CRD 60 & 5060 & Monroe & 5095 & Delta \\
\hline Arkansas & Arkansas CRD 70 & 5070 & Montgomery & 5097 & Delta \\
\hline Arkansas & Arkansas CRD 80 & 5080 & Nevada & 5099 & Delta \\
\hline Arkansas & Arkansas CRD 10 & 5010 & Newton & 5101 & Delta \\
\hline Arkansas & Arkansas CRD 80 & 5080 & Ouachita & 5103 & Delta \\
\hline Arkansas & Arkansas CRD 50 & 5050 & Perry & 5105 & Delta \\
\hline Arkansas & Arkansas CRD 60 & 5060 & Phillips & 5107 & Delta \\
\hline Arkansas & Arkansas CRD 70 & 5070 & Pike & 5109 & Delta \\
\hline Arkansas & Arkansas CRD 30 & 5030 & Poinsett & 5111 & Delta \\
\hline Arkansas & Arkansas CRD 40 & 5040 & Polk & 5113 & Delta \\
\hline Arkansas & Arkansas CRD 40 & 5040 & Pope & 5115 & Delta \\
\hline Arkansas & Arkansas CRD 60 & 5060 & Prairie & 5117 & Delta \\
\hline Arkansas & Arkansas CRD 50 & 5050 & Pulaski & 5119 & Delta \\
\hline Arkansas & Arkansas CRD 30 & 5030 & Randolph & 5121 & Delta \\
\hline Arkansas & Arkansas CRD 60 & 5060 & St. Francis & 5123 & Delta \\
\hline Arkansas & Arkansas CRD 50 & 5050 & Saline & 5125 & Delta \\
\hline Arkansas & Arkansas CRD 40 & 5040 & Scott & 5127 & Delta \\
\hline Arkansas & Arkansas CRD 20 & 5020 & Searcy & 5129 & Delta \\
\hline Arkansas & Arkansas CRD 40 & 5040 & Sebastian & 5131 & Delta \\
\hline Arkansas & Arkansas CRD 70 & 5070 & Sevier & 5133 & Delta \\
\hline Arkansas & Arkansas CRD 20 & 5020 & Sharp & 5135 & Delta \\
\hline Arkansas & Arkansas CRD 20 & 5020 & Stone & 5137 & Delta \\
\hline Arkansas & Arkansas CRD 80 & 5080 & Union & 5139 & Delta \\
\hline Arkansas & Arkansas CRD 20 & 5020 & Van Buren & 5141 & Delta \\
\hline Arkansas & Arkansas CRD 10 & 5010 & Washington & 5143 & Delta \\
\hline Arkansas & Arkansas CRD 30 & 5030 & White & 5145 & Delta \\
\hline Arkansas & Arkansas CRD 60 & 5060 & Woodruff & 5147 & Delta \\
\hline Arkansas & Arkansas CRD 40 & 5040 & Yell & 5149 & Delta \\
\hline California & California CRD 40 & 6040 & Alameda & 6001 & Pacific \\
\hline California & California CRD 60 & 6060 & Alpine & 6003 & Pacific \\
\hline California & California CRD 60 & 6060 & Amador & 6005 & Pacific \\
\hline California & California CRD 50 & 6050 & Butte & 6007 & Pacific \\
\hline California & California CRD 60 & 6060 & Calaveras & 6009 & Pacific \\
\hline California & California CRD 50 & 6050 & Colusa & 6011 & Pacific \\
\hline California & California CRD 40 & 6040 & Contra Costa & 6013 & Pacific \\
\hline California & California CRD 10 & 6010 & Del Norte & 6015 & Pacific \\
\hline California & California CRD 60 & 6060 & El Dorado & 6017 & Pacific \\
\hline California & California CRD 51 & 6051 & Fresno & 6019 & Pacific \\
\hline California & California CRD 50 & 6050 & Glenn & 6021 & Pacific \\
\hline
\end{tabular}


Table 1. Crop Reporting Districts (CRD) of the conterminous United States and associated counties.-Continued

[FIPS, Federal Information Processing Standard; USDA, U.S. Department of Agriculture]

\begin{tabular}{|c|c|c|c|c|c|}
\hline State & $\begin{array}{l}\text { Crop Reporting District } \\
\text { (CRD) }\end{array}$ & CRD Identifier & County Name & County FIPS & $\begin{array}{c}\text { USDA Farm Production } \\
\text { Region }\end{array}$ \\
\hline California & California CRD 10 & 6010 & Humboldt & 6023 & Pacific \\
\hline California & California CRD 80 & 6080 & Imperial & 6025 & Pacific \\
\hline California & California CRD 60 & 6060 & Inyo & 6027 & Pacific \\
\hline California & California CRD 51 & 6051 & Kern & 6029 & Pacific \\
\hline California & California CRD 51 & 6051 & Kings & 6031 & Pacific \\
\hline California & California CRD 40 & 6040 & Lake & 6033 & Pacific \\
\hline California & California CRD 30 & 6030 & Lassen & 6035 & Pacific \\
\hline California & California CRD 80 & 6080 & Los Angeles & 6037 & Pacific \\
\hline California & California CRD 51 & 6051 & Madera & 6039 & Pacific \\
\hline California & California CRD 40 & 6040 & Marin & 6041 & Pacific \\
\hline California & California CRD 60 & 6060 & Mariposa & 6043 & Pacific \\
\hline California & California CRD 10 & 6010 & Mendocino & 6045 & Pacific \\
\hline California & California CRD 51 & 6051 & Merced & 6047 & Pacific \\
\hline California & California CRD 30 & 6030 & Modoc & 6049 & Pacific \\
\hline California & California CRD 60 & 6060 & Mono & 6051 & Pacific \\
\hline California & California CRD 40 & 6040 & Monterey & 6053 & Pacific \\
\hline California & California CRD 40 & 6040 & Napa & 6055 & Pacific \\
\hline California & California CRD 60 & 6060 & Nevada & 6057 & Pacific \\
\hline California & California CRD 80 & 6080 & Orange & 6059 & Pacific \\
\hline California & California CRD 60 & 6060 & Placer & 6061 & Pacific \\
\hline California & California CRD 30 & 6030 & Plumas & 6063 & Pacific \\
\hline California & California CRD 80 & 6080 & Riverside & 6065 & Pacific \\
\hline California & California CRD 50 & 6050 & Sacramento & 6067 & Pacific \\
\hline California & California CRD 40 & 6040 & San Benito & 6069 & Pacific \\
\hline California & California CRD 80 & 6080 & San Bernardino & 6071 & Pacific \\
\hline California & California CRD 80 & 6080 & San Diego & 6073 & Pacific \\
\hline California & California CRD 40 & 6040 & San Francisco & 6075 & Pacific \\
\hline California & California CRD 51 & 6051 & San Joaquin & 6077 & Pacific \\
\hline California & California CRD 40 & 6040 & San Luis Obispo & 6079 & Pacific \\
\hline California & California CRD 40 & 6040 & San Mateo & 6081 & Pacific \\
\hline California & California CRD 80 & 6080 & Santa Barbara & 6083 & Pacific \\
\hline California & California CRD 40 & 6040 & Santa Clara & 6085 & Pacific \\
\hline California & California CRD 40 & 6040 & Santa Cruz & 6087 & Pacific \\
\hline California & California CRD 20 & 6020 & Shasta & 6089 & Pacific \\
\hline California & California CRD 60 & 6060 & Sierra & 6091 & Pacific \\
\hline California & California CRD 20 & 6020 & Siskiyou & 6093 & Pacific \\
\hline California & California CRD 50 & 6050 & Solano & 6095 & Pacific \\
\hline California & California CRD 40 & 6040 & Sonoma & 6097 & Pacific \\
\hline California & California CRD 51 & 6051 & Stanislaus & 6099 & Pacific \\
\hline California & California CRD 50 & 6050 & Sutter & 6101 & Pacific \\
\hline California & California CRD 50 & 6050 & Tehama & 6103 & Pacific \\
\hline California & California CRD 20 & 6020 & Trinity & 6105 & Pacific \\
\hline
\end{tabular}


Table 1. Crop Reporting Districts (CRD) of the conterminous United States and associated counties. - Continued

[FIPS, Federal Information Processing Standard; USDA, U.S. Department of Agriculture]

\begin{tabular}{|c|c|c|c|c|c|}
\hline State & $\begin{array}{l}\text { Crop Reporting District } \\
\text { (CRD) }\end{array}$ & CRD Identifier & County Name & County FIPS & $\begin{array}{c}\text { USDA Farm Production } \\
\text { Region }\end{array}$ \\
\hline California & California CRD 51 & 6051 & Tulare & 6107 & Pacific \\
\hline California & California CRD 60 & 6060 & Tuolumne & 6109 & Pacific \\
\hline California & California CRD 80 & 6080 & Ventura & 6111 & Pacific \\
\hline California & California CRD 50 & 6050 & Yolo & 6113 & Pacific \\
\hline California & California CRD 50 & 6050 & Yuba & 6115 & Pacific \\
\hline Colorado & Colorado CRD 60 & 8060 & Adams & 8001 & Mountain \\
\hline Colorado & Colorado CRD 80 & 8080 & Alamosa & 8003 & Mountain \\
\hline Colorado & Colorado CRD 60 & 8060 & Arapahoe & 8005 & Mountain \\
\hline Colorado & Colorado CRD 70 & 8070 & Archuleta & 8007 & Mountain \\
\hline Colorado & Colorado CRD 90 & 8090 & Baca & 8009 & Mountain \\
\hline Colorado & Colorado CRD 90 & 8090 & Bent & 8011 & Mountain \\
\hline Colorado & Colorado CRD 20 & 8020 & Boulder & 8013 & Mountain \\
\hline Colorado & Colorado CRD 60 & 8060 & Broomfield & 8014 & Mountain \\
\hline Colorado & Colorado CRD 10 & 8010 & Chaffee & 8015 & Mountain \\
\hline Colorado & Colorado CRD 60 & 8060 & Cheyenne & 8017 & Mountain \\
\hline Colorado & Colorado CRD 10 & 8010 & Clear Creek & 8019 & Mountain \\
\hline Colorado & Colorado CRD 80 & 8080 & Conejos & 8021 & Mountain \\
\hline Colorado & Colorado CRD 80 & 8080 & Costilla & 8023 & Mountain \\
\hline Colorado & Colorado CRD 90 & 8090 & Crowley & 8025 & Mountain \\
\hline Colorado & Colorado CRD 90 & 8090 & Custer & 8027 & Mountain \\
\hline Colorado & Colorado CRD 70 & 8070 & Delta & 8029 & Mountain \\
\hline Colorado & Colorado CRD 60 & 8060 & Denver & 8031 & Mountain \\
\hline Colorado & Colorado CRD 70 & 8070 & Dolores & 8033 & Mountain \\
\hline Colorado & Colorado CRD 60 & 8060 & Douglas & 8035 & Mountain \\
\hline Colorado & Colorado CRD 10 & 8010 & Eagle & 8037 & Mountain \\
\hline Colorado & Colorado CRD 60 & 8060 & Elbert & 8039 & Mountain \\
\hline Colorado & Colorado CRD 60 & 8060 & El Paso & 8041 & Mountain \\
\hline Colorado & Colorado CRD 90 & 8090 & Fremont & 8043 & Mountain \\
\hline Colorado & Colorado CRD 70 & 8070 & Garfield & 8045 & Mountain \\
\hline Colorado & Colorado CRD 10 & 8010 & Gilpin & 8047 & Mountain \\
\hline Colorado & Colorado CRD 10 & 8010 & Grand & 8049 & Mountain \\
\hline Colorado & Colorado CRD 10 & 8010 & Gunnison & 8051 & Mountain \\
\hline Colorado & Colorado CRD 70 & 8070 & Hinsdale & 8053 & Mountain \\
\hline Colorado & Colorado CRD 90 & 8090 & Huerfano & 8055 & Mountain \\
\hline Colorado & Colorado CRD 10 & 8010 & Jackson & 8057 & Mountain \\
\hline Colorado & Colorado CRD 20 & 8020 & Jefferson & 8059 & Mountain \\
\hline Colorado & Colorado CRD 60 & 8060 & Kiowa & 8061 & Mountain \\
\hline Colorado & Colorado CRD 60 & 8060 & Kit Carson & 8063 & Mountain \\
\hline Colorado & Colorado CRD 10 & 8010 & Lake & 8065 & Mountain \\
\hline Colorado & Colorado CRD 70 & 8070 & La Plata & 8067 & Mountain \\
\hline Colorado & Colorado CRD 20 & 8020 & Larimer & 8069 & Mountain \\
\hline Colorado & Colorado CRD 90 & 8090 & Las Animas & 8071 & Mountain \\
\hline
\end{tabular}


Table 1. Crop Reporting Districts (CRD) of the conterminous United States and associated counties.-Continued

[FIPS, Federal Information Processing Standard; USDA, U.S. Department of Agriculture]

\begin{tabular}{|c|c|c|c|c|c|}
\hline State & $\begin{array}{l}\text { Crop Reporting District } \\
\text { (CRD) }\end{array}$ & CRD Identifier & County Name & County FIPS & $\begin{array}{c}\text { USDA Farm Production } \\
\text { Region }\end{array}$ \\
\hline Colorado & Colorado CRD 60 & 8060 & Lincoln & 8073 & Mountain \\
\hline Colorado & Colorado CRD 20 & 8020 & Logan & 8075 & Mountain \\
\hline Colorado & Colorado CRD 70 & 8070 & Mesa & 8077 & Mountain \\
\hline Colorado & Colorado CRD 80 & 8080 & Mineral & 8079 & Mountain \\
\hline Colorado & Colorado CRD 10 & 8010 & Moffat & 8081 & Mountain \\
\hline Colorado & Colorado CRD 70 & 8070 & Montezuma & 8083 & Mountain \\
\hline Colorado & Colorado CRD 70 & 8070 & Montrose & 8085 & Mountain \\
\hline Colorado & Colorado CRD 20 & 8020 & Morgan & 8087 & Mountain \\
\hline Colorado & Colorado CRD 90 & 8090 & Otero & 8089 & Mountain \\
\hline Colorado & Colorado CRD 70 & 8070 & Ouray & 8091 & Mountain \\
\hline Colorado & Colorado CRD 10 & 8010 & Park & 8093 & Mountain \\
\hline Colorado & Colorado CRD 60 & 8060 & Phillips & 8095 & Mountain \\
\hline Colorado & Colorado CRD 10 & 8010 & Pitkin & 8097 & Mountain \\
\hline Colorado & Colorado CRD 90 & 8090 & Prowers & 8099 & Mountain \\
\hline Colorado & Colorado CRD 90 & 8090 & Pueblo & 8101 & Mountain \\
\hline Colorado & Colorado CRD 10 & 8010 & Rio Blanco & 8103 & Mountain \\
\hline Colorado & Colorado CRD 80 & 8080 & Rio Grande & 8105 & Mountain \\
\hline Colorado & Colorado CRD 10 & 8010 & Routt & 8107 & Mountain \\
\hline Colorado & Colorado CRD 80 & 8080 & Saguache & 8109 & Mountain \\
\hline Colorado & Colorado CRD 70 & 8070 & San Juan & 8111 & Mountain \\
\hline Colorado & Colorado CRD 70 & 8070 & San Miguel & 8113 & Mountain \\
\hline Colorado & Colorado CRD 20 & 8020 & Sedgwick & 8115 & Mountain \\
\hline Colorado & Colorado CRD 10 & 8010 & Summit & 8117 & Mountain \\
\hline Colorado & Colorado CRD 10 & 8010 & Teller & 8119 & Mountain \\
\hline Colorado & Colorado CRD 60 & 8060 & Washington & 8121 & Mountain \\
\hline Colorado & Colorado CRD 20 & 8020 & Weld & 8123 & Mountain \\
\hline Colorado & Colorado CRD 60 & 8060 & Yuma & 8125 & Mountain \\
\hline Connecticut & Connecticut CRD 10 & 9010 & Fairfield & 9001 & Northeast \\
\hline Connecticut & Connecticut CRD 10 & 9010 & Hartford & 9003 & Northeast \\
\hline Connecticut & Connecticut CRD 10 & 9010 & Litchfield & 9005 & Northeast \\
\hline Connecticut & Connecticut CRD 10 & 9010 & Middlesex & 9007 & Northeast \\
\hline Connecticut & Connecticut CRD 10 & 9010 & New Haven & 9009 & Northeast \\
\hline Connecticut & Connecticut CRD 10 & 9010 & New London & 9011 & Northeast \\
\hline Connecticut & Connecticut CRD 10 & 9010 & Tolland & 9013 & Northeast \\
\hline Connecticut & Connecticut CRD 10 & 9010 & Windham & 9015 & Northeast \\
\hline Delaware & Delaware CRD 50 & 10050 & Kent & 10001 & Northeast \\
\hline Delaware & Delaware CRD 20 & 10020 & New Castle & 10003 & Northeast \\
\hline Delaware & Delaware CRD 80 & 10080 & Sussex & 10005 & Northeast \\
\hline Florida & Florida CRD 50 & 12050 & Alachua & 12001 & Southeast \\
\hline Florida & Florida CRD 30 & 12030 & Baker & 12003 & Southeast \\
\hline Florida & Florida CRD 10 & 12010 & Bay & 12005 & Southeast \\
\hline Florida & Florida CRD 50 & 12050 & Bradford & 12007 & Southeast \\
\hline
\end{tabular}


Table 1. Crop Reporting Districts (CRD) of the conterminous United States and associated counties.-Continued

[FIPS, Federal Information Processing Standard; USDA, U.S. Department of Agriculture]

\begin{tabular}{|c|c|c|c|c|c|}
\hline State & $\begin{array}{l}\text { Crop Reporting District } \\
\text { (CRD) }\end{array}$ & CRD Identifier & County Name & County FIPS & $\begin{array}{c}\text { USDA Farm Production } \\
\text { Region }\end{array}$ \\
\hline Florida & Florida CRD 80 & 12080 & Brevard & 12009 & Southeast \\
\hline Florida & Florida CRD 80 & 12080 & Broward & 12011 & Southeast \\
\hline Florida & Florida CRD 10 & 12010 & Calhoun & 12013 & Southeast \\
\hline Florida & Florida CRD 80 & 12080 & Charlotte & 12015 & Southeast \\
\hline Florida & Florida CRD 50 & 12050 & Citrus & 12017 & Southeast \\
\hline Florida & Florida CRD 50 & 12050 & Clay & 12019 & Southeast \\
\hline Florida & Florida CRD 80 & 12080 & Collier & 12021 & Southeast \\
\hline Florida & Florida CRD 30 & 12030 & Columbia & 12023 & Southeast \\
\hline Florida & Florida CRD 80 & 12080 & De Soto & 12027 & Southeast \\
\hline Florida & Florida CRD 30 & 12030 & Dixie & 12029 & Southeast \\
\hline Florida & Florida CRD 30 & 12030 & Duval & 12031 & Southeast \\
\hline Florida & Florida CRD 10 & 12010 & Escambia & 12033 & Southeast \\
\hline Florida & Florida CRD 50 & 12050 & Flagler & 12035 & Southeast \\
\hline Florida & Florida CRD 10 & 12010 & Franklin & 12037 & Southeast \\
\hline Florida & Florida CRD 10 & 12010 & Gadsden & 12039 & Southeast \\
\hline Florida & Florida CRD 50 & 12050 & Gilchrist & 12041 & Southeast \\
\hline Florida & Florida CRD 80 & 12080 & Glades & 12043 & Southeast \\
\hline Florida & Florida CRD 10 & 12010 & Gulf & 12045 & Southeast \\
\hline Florida & Florida CRD 30 & 12030 & Hamilton & 12047 & Southeast \\
\hline Florida & Florida CRD 80 & 12080 & Hardee & 12049 & Southeast \\
\hline Florida & Florida CRD 80 & 12080 & Hendry & 12051 & Southeast \\
\hline Florida & Florida CRD 50 & 12050 & Hernando & 12053 & Southeast \\
\hline Florida & Florida CRD 80 & 12080 & Highlands & 12055 & Southeast \\
\hline Florida & Florida CRD 50 & 12050 & Hillsborough & 12057 & Southeast \\
\hline Florida & Florida CRD 10 & 12010 & Holmes & 12059 & Southeast \\
\hline Florida & Florida CRD 80 & 12080 & Indian River & 12061 & Southeast \\
\hline Florida & Florida CRD 10 & 12010 & Jackson & 12063 & Southeast \\
\hline Florida & Florida CRD 10 & 12010 & Jefferson & 12065 & Southeast \\
\hline Florida & Florida CRD 30 & 12030 & Lafayette & 12067 & Southeast \\
\hline Florida & Florida CRD 50 & 12050 & Lake & 12069 & Southeast \\
\hline Florida & Florida CRD 80 & 12080 & Lee & 12071 & Southeast \\
\hline Florida & Florida CRD 10 & 12010 & Leon & 12073 & Southeast \\
\hline Florida & Florida CRD 50 & 12050 & Levy & 12075 & Southeast \\
\hline Florida & Florida CRD 10 & 12010 & Liberty & 12077 & Southeast \\
\hline Florida & Florida CRD 30 & 12030 & Madison & 12079 & Southeast \\
\hline Florida & Florida CRD 80 & 12080 & Manatee & 12081 & Southeast \\
\hline Florida & Florida CRD 50 & 12050 & Marion & 12083 & Southeast \\
\hline Florida & Florida CRD 80 & 12080 & Martin & 12085 & Southeast \\
\hline Florida & Florida CRD 80 & 12080 & Miami-Dade & 12086 & Southeast \\
\hline Florida & Florida CRD 80 & 12080 & Monroe & 12087 & Southeast \\
\hline Florida & Florida CRD 30 & 12030 & Nassau & 12089 & Southeast \\
\hline Florida & Florida CRD 10 & 12010 & Okaloosa & 12091 & Southeast \\
\hline
\end{tabular}


Table 1. Crop Reporting Districts (CRD) of the conterminous United States and associated counties.—Continued

[FIPS, Federal Information Processing Standard; USDA, U.S. Department of Agriculture]

\begin{tabular}{|c|c|c|c|c|c|}
\hline State & $\begin{array}{l}\text { Crop Reporting District } \\
\text { (CRD) }\end{array}$ & CRD Identifier & County Name & County FIPS & $\begin{array}{c}\text { USDA Farm Production } \\
\text { Region }\end{array}$ \\
\hline Florida & Florida CRD 80 & 12080 & Okeechobee & 12093 & Southeast \\
\hline Florida & Florida CRD 50 & 12050 & Orange & 12095 & Southeast \\
\hline Florida & Florida CRD 50 & 12050 & Osceola & 12097 & Southeast \\
\hline Florida & Florida CRD 80 & 12080 & Palm Beach & 12099 & Southeast \\
\hline Florida & Florida CRD 50 & 12050 & Pasco & 12101 & Southeast \\
\hline Florida & Florida CRD 50 & 12050 & Pinellas & 12103 & Southeast \\
\hline Florida & Florida CRD 50 & 12050 & Polk & 12105 & Southeast \\
\hline Florida & Florida CRD 50 & 12050 & Putnam & 12107 & Southeast \\
\hline Florida & Florida CRD 50 & 12050 & St. Johns & 12109 & Southeast \\
\hline Florida & Florida CRD 80 & 12080 & St. Lucie & 12111 & Southeast \\
\hline Florida & Florida CRD 10 & 12010 & Santa Rosa & 12113 & Southeast \\
\hline Florida & Florida CRD 80 & 12080 & Sarasota & 12115 & Southeast \\
\hline Florida & Florida CRD 50 & 12050 & Seminole & 12117 & Southeast \\
\hline Florida & Florida CRD 50 & 12050 & Sumter & 12119 & Southeast \\
\hline Florida & Florida CRD 30 & 12030 & Suwannee & 12121 & Southeast \\
\hline Florida & Florida CRD 30 & 12030 & Taylor & 12123 & Southeast \\
\hline Florida & Florida CRD 50 & 12050 & Union & 12125 & Southeast \\
\hline Florida & Florida CRD 50 & 12050 & Volusia & 12127 & Southeast \\
\hline Florida & Florida CRD 10 & 12010 & Wakulla & 12129 & Southeast \\
\hline Florida & Florida CRD 10 & 12010 & Walton & 12131 & Southeast \\
\hline Florida & Florida CRD 10 & 12010 & Washington & 12133 & Southeast \\
\hline Georgia & Georgia CRD 90 & 13090 & Appling & 13001 & Southeast \\
\hline Georgia & Georgia CRD 80 & 13080 & Atkinson & 13003 & Southeast \\
\hline Georgia & Georgia CRD 90 & 13090 & Bacon & 13005 & Southeast \\
\hline Georgia & Georgia CRD 70 & 13070 & Baker & 13007 & Southeast \\
\hline Georgia & Georgia CRD 50 & 13050 & Baldwin & 13009 & Southeast \\
\hline Georgia & Georgia CRD 30 & 13030 & Banks & 13011 & Southeast \\
\hline Georgia & Georgia CRD 20 & 13020 & Barrow & 13013 & Southeast \\
\hline Georgia & Georgia CRD 10 & 13010 & Bartow & 13015 & Southeast \\
\hline Georgia & Georgia CRD 80 & 13080 & Ben hill & 13017 & Southeast \\
\hline Georgia & Georgia CRD 80 & 13080 & Berrien & 13019 & Southeast \\
\hline Georgia & Georgia CRD 50 & 13050 & Bibb & 13021 & Southeast \\
\hline Georgia & Georgia CRD 50 & 13050 & Bleckley & 13023 & Southeast \\
\hline Georgia & Georgia CRD 90 & 13090 & Brantley & 13025 & Southeast \\
\hline Georgia & Georgia CRD 80 & 13080 & Brooks & 13027 & Southeast \\
\hline Georgia & Georgia CRD 90 & 13090 & Bryan & 13029 & Southeast \\
\hline Georgia & Georgia CRD 60 & 13060 & Bulloch & 13031 & Southeast \\
\hline Georgia & Georgia CRD 60 & 13060 & Burke & 13033 & Southeast \\
\hline Georgia & Georgia CRD 50 & 13050 & Butts & 13035 & Southeast \\
\hline Georgia & Georgia CRD 70 & 13070 & Calhoun & 13037 & Southeast \\
\hline Georgia & Georgia CRD 90 & 13090 & Camden & 13039 & Southeast \\
\hline Georgia & Georgia CRD 60 & 13060 & Candler & 13043 & Southeast \\
\hline
\end{tabular}


Table 1. Crop Reporting Districts (CRD) of the conterminous United States and associated counties.-Continued

[FIPS, Federal Information Processing Standard; USDA, U.S. Department of Agriculture]

\begin{tabular}{|c|c|c|c|c|c|}
\hline State & $\begin{array}{l}\text { Crop Reporting District } \\
\text { (CRD) }\end{array}$ & CRD Identifier & County Name & County FIPS & $\begin{array}{c}\text { USDA Farm Production } \\
\text { Region }\end{array}$ \\
\hline Georgia & Georgia CRD 40 & 13040 & Carroll & 13045 & Southeast \\
\hline Georgia & Georgia CRD 10 & 13010 & Catoosa & 13047 & Southeast \\
\hline Georgia & Georgia CRD 90 & 13090 & Charlton & 13049 & Southeast \\
\hline Georgia & Georgia CRD 90 & 13090 & Chatham & 13051 & Southeast \\
\hline Georgia & Georgia CRD 40 & 13040 & Chattahoochee & 13053 & Southeast \\
\hline Georgia & Georgia CRD 10 & 13010 & Chattooga & 13055 & Southeast \\
\hline Georgia & Georgia CRD 20 & 13020 & Cherokee & 13057 & Southeast \\
\hline Georgia & Georgia CRD 20 & 13020 & Clarke & 13059 & Southeast \\
\hline Georgia & Georgia CRD 70 & 13070 & Clay & 13061 & Southeast \\
\hline Georgia & Georgia CRD 40 & 13040 & Clayton & 13063 & Southeast \\
\hline Georgia & Georgia CRD 80 & 13080 & Clinch & 13065 & Southeast \\
\hline Georgia & Georgia CRD 20 & 13020 & Cobb & 13067 & Southeast \\
\hline Georgia & Georgia CRD 80 & 13080 & Coffee & 13069 & Southeast \\
\hline Georgia & Georgia CRD 80 & 13080 & Colquitt & 13071 & Southeast \\
\hline Georgia & Georgia CRD 60 & 13060 & Columbia & 13073 & Southeast \\
\hline Georgia & Georgia CRD 80 & 13080 & Cook & 13075 & Southeast \\
\hline Georgia & Georgia CRD 40 & 13040 & Coweta & 13077 & Southeast \\
\hline Georgia & Georgia CRD 50 & 13050 & Crawford & 13079 & Southeast \\
\hline Georgia & Georgia CRD 80 & 13080 & Crisp & 13081 & Southeast \\
\hline Georgia & Georgia CRD 10 & 13010 & Dade & 13083 & Southeast \\
\hline Georgia & Georgia CRD 20 & 13020 & Dawson & 13085 & Southeast \\
\hline Georgia & Georgia CRD 70 & 13070 & Decatur & 13087 & Southeast \\
\hline Georgia & Georgia CRD 20 & 13020 & De Kalb & 13089 & Southeast \\
\hline Georgia & Georgia CRD 50 & 13050 & Dodge & 13091 & Southeast \\
\hline Georgia & Georgia CRD 80 & 13080 & Dooly & 13093 & Southeast \\
\hline Georgia & Georgia CRD 70 & 13070 & Dougherty & 13095 & Southeast \\
\hline Georgia & Georgia CRD 40 & 13040 & Douglas & 13097 & Southeast \\
\hline Georgia & Georgia CRD 70 & 13070 & Early & 13099 & Southeast \\
\hline Georgia & Georgia CRD 80 & 13080 & Echols & 13101 & Southeast \\
\hline Georgia & Georgia CRD 60 & 13060 & Effingham & 13103 & Southeast \\
\hline Georgia & Georgia CRD 30 & 13030 & Elbert & 13105 & Southeast \\
\hline Georgia & Georgia CRD 60 & 13060 & Emanuel & 13107 & Southeast \\
\hline Georgia & Georgia CRD 90 & 13090 & Evans & 13109 & Southeast \\
\hline Georgia & Georgia CRD 20 & 13020 & Fannin & 13111 & Southeast \\
\hline Georgia & Georgia CRD 40 & 13040 & Fayette & 13113 & Southeast \\
\hline Georgia & Georgia CRD 10 & 13010 & Floyd & 13115 & Southeast \\
\hline Georgia & Georgia CRD 20 & 13020 & Forsyth & 13117 & Southeast \\
\hline Georgia & Georgia CRD 30 & 13030 & Franklin & 13119 & Southeast \\
\hline Georgia & Georgia CRD 20 & 13020 & Fulton & 13121 & Southeast \\
\hline Georgia & Georgia CRD 20 & 13020 & Gilmer & 13123 & Southeast \\
\hline Georgia & Georgia CRD 60 & 13060 & Glascock & 13125 & Southeast \\
\hline Georgia & Georgia CRD 90 & 13090 & Glynn & 13127 & Southeast \\
\hline
\end{tabular}


Table 1. Crop Reporting Districts (CRD) of the conterminous United States and associated counties.-Continued

[FIPS, Federal Information Processing Standard; USDA, U.S. Department of Agriculture]

\begin{tabular}{|c|c|c|c|c|c|}
\hline State & $\begin{array}{l}\text { Crop Reporting District } \\
\text { (CRD) }\end{array}$ & CRD Identifier & County Name & County FIPS & $\begin{array}{c}\text { USDA Farm Production } \\
\text { Region }\end{array}$ \\
\hline Georgia & Georgia CRD 10 & 13010 & Gordon & 13129 & Southeast \\
\hline Georgia & Georgia CRD 70 & 13070 & Grady & 13131 & Southeast \\
\hline Georgia & Georgia CRD 50 & 13050 & Greene & 13133 & Southeast \\
\hline Georgia & Georgia CRD 20 & 13020 & Gwinnett & 13135 & Southeast \\
\hline Georgia & Georgia CRD 30 & 13030 & Habersham & 13137 & Southeast \\
\hline Georgia & Georgia CRD 20 & 13020 & Hall & 13139 & Southeast \\
\hline Georgia & Georgia CRD 50 & 13050 & Hancock & 13141 & Southeast \\
\hline Georgia & Georgia CRD 40 & 13040 & Haralson & 13143 & Southeast \\
\hline Georgia & Georgia CRD 40 & 13040 & Harris & 13145 & Southeast \\
\hline Georgia & Georgia CRD 30 & 13030 & Hart & 13147 & Southeast \\
\hline Georgia & Georgia CRD 40 & 13040 & Heard & 13149 & Southeast \\
\hline Georgia & Georgia CRD 40 & 13040 & Henry & 13151 & Southeast \\
\hline Georgia & Georgia CRD 50 & 13050 & Houston & 13153 & Southeast \\
\hline Georgia & Georgia CRD 80 & 13080 & Irwin & 13155 & Southeast \\
\hline Georgia & Georgia CRD 20 & 13020 & Jackson & 13157 & Southeast \\
\hline Georgia & Georgia CRD 50 & 13050 & Jasper & 13159 & Southeast \\
\hline Georgia & Georgia CRD 80 & 13080 & Jeff Davis & 13161 & Southeast \\
\hline Georgia & Georgia CRD 60 & 13060 & Jefferson & 13163 & Southeast \\
\hline Georgia & Georgia CRD 60 & 13060 & Jenkins & 13165 & Southeast \\
\hline Georgia & Georgia CRD 50 & 13050 & Johnson & 13167 & Southeast \\
\hline Georgia & Georgia CRD 50 & 13050 & Jones & 13169 & Southeast \\
\hline Georgia & Georgia CRD 40 & 13040 & Lamar & 13171 & Southeast \\
\hline Georgia & Georgia CRD 80 & 13080 & Lanier & 13173 & Southeast \\
\hline Georgia & Georgia CRD 50 & 13050 & Laurens & 13175 & Southeast \\
\hline Georgia & Georgia CRD 70 & 13070 & Lee & 13177 & Southeast \\
\hline Georgia & Georgia CRD 90 & 13090 & Liberty & 13179 & Southeast \\
\hline Georgia & Georgia CRD 30 & 13030 & Lincoln & 13181 & Southeast \\
\hline Georgia & Georgia CRD 90 & 13090 & Long & 13183 & Southeast \\
\hline Georgia & Georgia CRD 80 & 13080 & Lowndes & 13185 & Southeast \\
\hline Georgia & Georgia CRD 20 & 13020 & Lumpkin & 13187 & Southeast \\
\hline Georgia & Georgia CRD 60 & 13060 & McDuffie & 13189 & Southeast \\
\hline Georgia & Georgia CRD 90 & 13090 & McIntosh & 13191 & Southeast \\
\hline Georgia & Georgia CRD 40 & 13040 & Macon & 13193 & Southeast \\
\hline Georgia & Georgia CRD 30 & 13030 & Madison & 13195 & Southeast \\
\hline Georgia & Georgia CRD 40 & 13040 & Marion & 13197 & Southeast \\
\hline Georgia & Georgia CRD 40 & 13040 & Meriwether & 13199 & Southeast \\
\hline Georgia & Georgia CRD 70 & 13070 & Miller & 13201 & Southeast \\
\hline Georgia & Georgia CRD 70 & 13070 & Mitchell & 13205 & Southeast \\
\hline Georgia & Georgia CRD 50 & 13050 & Monroe & 13207 & Southeast \\
\hline Georgia & Georgia CRD 50 & 13050 & Montgomery & 13209 & Southeast \\
\hline Georgia & Georgia CRD 50 & 13050 & Morgan & 13211 & Southeast \\
\hline Georgia & Georgia CRD 10 & 13010 & Murray & 13213 & Southeast \\
\hline
\end{tabular}


Table 1. Crop Reporting Districts (CRD) of the conterminous United States and associated counties. - Continued

[FIPS, Federal Information Processing Standard; USDA, U.S. Department of Agriculture]

\begin{tabular}{|c|c|c|c|c|c|}
\hline State & $\begin{array}{l}\text { Crop Reporting District } \\
\text { (CRD) }\end{array}$ & CRD Identifier & County Name & County FIPS & $\begin{array}{l}\text { USDA Farm Production } \\
\text { Region }\end{array}$ \\
\hline Georgia & Georgia CRD 40 & 13040 & Muscogee & 13215 & Southeast \\
\hline Georgia & Georgia CRD 50 & 13050 & Newton & 13217 & Southeast \\
\hline Georgia & Georgia CRD 20 & 13020 & Oconee & 13219 & Southeast \\
\hline Georgia & Georgia CRD 30 & 13030 & Oglethorpe & 13221 & Southeast \\
\hline Georgia & Georgia CRD 10 & 13010 & Paulding & 13223 & Southeast \\
\hline Georgia & Georgia CRD 50 & 13050 & Peach & 13225 & Southeast \\
\hline Georgia & Georgia CRD 20 & 13020 & Pickens & 13227 & Southeast \\
\hline Georgia & Georgia CRD 90 & 13090 & Pierce & 13229 & Southeast \\
\hline Georgia & Georgia CRD 40 & 13040 & Pike & 13231 & Southeast \\
\hline Georgia & Georgia CRD 10 & 13010 & Polk & 13233 & Southeast \\
\hline Georgia & Georgia CRD 50 & 13050 & Pulaski & 13235 & Southeast \\
\hline Georgia & Georgia CRD 50 & 13050 & Putnam & 13237 & Southeast \\
\hline Georgia & Georgia CRD 70 & 13070 & Quitman & 13239 & Southeast \\
\hline Georgia & Georgia CRD 30 & 13030 & Rabun & 13241 & Southeast \\
\hline Georgia & Georgia CRD 70 & 13070 & Randolph & 13243 & Southeast \\
\hline Georgia & Georgia CRD 60 & 13060 & Richmond & 13245 & Southeast \\
\hline Georgia & Georgia CRD 50 & 13050 & Rockdale & 13247 & Southeast \\
\hline Georgia & Georgia CRD 40 & 13040 & Schley & 13249 & Southeast \\
\hline Georgia & Georgia CRD 60 & 13060 & Screven & 13251 & Southeast \\
\hline Georgia & Georgia CRD 70 & 13070 & Seminole & 13253 & Southeast \\
\hline Georgia & Georgia CRD 40 & 13040 & Spaulding & 13255 & Southeast \\
\hline Georgia & Georgia CRD 30 & 13030 & Stephens & 13257 & Southeast \\
\hline Georgia & Georgia CRD 70 & 13070 & Stewart & 13259 & Southeast \\
\hline Georgia & Georgia CRD 70 & 13070 & Sumter & 13261 & Southeast \\
\hline Georgia & Georgia CRD 40 & 13040 & Talbot & 13263 & Southeast \\
\hline Georgia & Georgia CRD 50 & 13050 & Taliaferro & 13265 & Southeast \\
\hline Georgia & Georgia CRD 90 & 13090 & Tattnall & 13267 & Southeast \\
\hline Georgia & Georgia CRD 40 & 13040 & Taylor & 13269 & Southeast \\
\hline Georgia & Georgia CRD 80 & 13080 & Telfair & 13271 & Southeast \\
\hline Georgia & Georgia CRD 70 & 13070 & Terrell & 13273 & Southeast \\
\hline Georgia & Georgia CRD 70 & 13070 & Thomas & 13275 & Southeast \\
\hline Georgia & Georgia CRD 80 & 13080 & Tift & 13277 & Southeast \\
\hline Georgia & Georgia CRD 90 & 13090 & Toombs & 13279 & Southeast \\
\hline Georgia & Georgia CRD 20 & 13020 & Towns & 13281 & Southeast \\
\hline Georgia & Georgia CRD 50 & 13050 & Treutlen & 13283 & Southeast \\
\hline Georgia & Georgia CRD 40 & 13040 & Troup & 13285 & Southeast \\
\hline Georgia & Georgia CRD 80 & 13080 & Turner & 13287 & Southeast \\
\hline Georgia & Georgia CRD 50 & 13050 & Twiggs & 13289 & Southeast \\
\hline Georgia & Georgia CRD 20 & 13020 & Union & 13291 & Southeast \\
\hline Georgia & Georgia CRD 40 & 13040 & Upson & 13293 & Southeast \\
\hline Georgia & Georgia CRD 10 & 13010 & Walker & 13295 & Southeast \\
\hline Georgia & Georgia CRD 20 & 13020 & Walton & 13297 & Southeast \\
\hline
\end{tabular}


Table 1. Crop Reporting Districts (CRD) of the conterminous United States and associated counties.-Continued

[FIPS, Federal Information Processing Standard; USDA, U.S. Department of Agriculture]

\begin{tabular}{|c|c|c|c|c|c|}
\hline State & $\begin{array}{l}\text { Crop Reporting District } \\
\text { (CRD) }\end{array}$ & CRD Identifier & County Name & County FIPS & $\begin{array}{l}\text { USDA Farm Production } \\
\text { Region }\end{array}$ \\
\hline Georgia & Georgia CRD 90 & 13090 & Ware & 13299 & Southeast \\
\hline Georgia & Georgia CRD 60 & 13060 & Warren & 13301 & Southeast \\
\hline Georgia & Georgia CRD 50 & 13050 & Washington & 13303 & Southeast \\
\hline Georgia & Georgia CRD 90 & 13090 & Wayne & 13305 & Southeast \\
\hline Georgia & Georgia CRD 70 & 13070 & Webster & 13307 & Southeast \\
\hline Georgia & Georgia CRD 50 & 13050 & Wheeler & 13309 & Southeast \\
\hline Georgia & Georgia CRD 20 & 13020 & White & 13311 & Southeast \\
\hline Georgia & Georgia CRD 10 & 13010 & Whitfield & 13313 & Southeast \\
\hline Georgia & Georgia CRD 80 & 13080 & Wilcox & 13315 & Southeast \\
\hline Georgia & Georgia CRD 30 & 13030 & Wilkes & 13317 & Southeast \\
\hline Georgia & Georgia CRD 50 & 13050 & Wilkinson & 13319 & Southeast \\
\hline Georgia & Georgia CRD 80 & 13080 & Worth & 13321 & Southeast \\
\hline Idaho & Idaho CRD 70 & 16070 & Ada & 16001 & Mountain \\
\hline Idaho & Idaho CRD 70 & 16070 & Adams & 16003 & Mountain \\
\hline Idaho & Idaho CRD 90 & 16090 & Bannock & 16005 & Mountain \\
\hline Idaho & Idaho CRD 90 & 16090 & Bear Lake & 16007 & Mountain \\
\hline Idaho & Idaho CRD 10 & 16010 & Benewah & 16009 & Mountain \\
\hline Idaho & Idaho CRD 90 & 16090 & Bingham & 16011 & Mountain \\
\hline Idaho & Idaho CRD 80 & 16080 & Blaine & 16013 & Mountain \\
\hline Idaho & Idaho CRD 70 & 16070 & Boise & 16015 & Mountain \\
\hline Idaho & Idaho CRD 10 & 16010 & Bonner & 16017 & Mountain \\
\hline Idaho & Idaho CRD 90 & 16090 & Bonneville & 16019 & Mountain \\
\hline Idaho & Idaho CRD 10 & 16010 & Boundary & 16021 & Mountain \\
\hline Idaho & Idaho CRD 90 & 16090 & Butte & 16023 & Mountain \\
\hline Idaho & Idaho CRD 80 & 16080 & Camas & 16025 & Mountain \\
\hline Idaho & Idaho CRD 70 & 16070 & Canyon & 16027 & Mountain \\
\hline Idaho & Idaho CRD 90 & 16090 & Caribou & 16029 & Mountain \\
\hline Idaho & Idaho CRD 80 & 16080 & Cassia & 16031 & Mountain \\
\hline Idaho & Idaho CRD 90 & 16090 & Clark & 16033 & Mountain \\
\hline Idaho & Idaho CRD 10 & 16010 & Clearwater & 16035 & Mountain \\
\hline Idaho & Idaho CRD 90 & 16090 & Custer & 16037 & Mountain \\
\hline Idaho & Idaho CRD 70 & 16070 & Elmore & 16039 & Mountain \\
\hline Idaho & Idaho CRD 90 & 16090 & Franklin & 16041 & Mountain \\
\hline Idaho & Idaho CRD 90 & 16090 & Fremont & 16043 & Mountain \\
\hline Idaho & Idaho CRD 70 & 16070 & Gem & 16045 & Mountain \\
\hline Idaho & Idaho CRD 80 & 16080 & Gooding & 16047 & Mountain \\
\hline Idaho & Idaho CRD 10 & 16010 & Idaho & 16049 & Mountain \\
\hline Idaho & Idaho CRD 90 & 16090 & Jefferson & 16051 & Mountain \\
\hline Idaho & Idaho CRD 80 & 16080 & Jerome & 16053 & Mountain \\
\hline Idaho & Idaho CRD 10 & 16010 & Kootenai & 16055 & Mountain \\
\hline Idaho & Idaho CRD 10 & 16010 & Latah & 16057 & Mountain \\
\hline Idaho & Idaho CRD 90 & 16090 & Lemhi & 16059 & Mountain \\
\hline
\end{tabular}


Table 1. Crop Reporting Districts (CRD) of the conterminous United States and associated counties.-Continued

[FIPS, Federal Information Processing Standard; USDA, U.S. Department of Agriculture]

\begin{tabular}{|c|c|c|c|c|c|}
\hline State & $\begin{array}{l}\text { Crop Reporting District } \\
\text { (CRD) }\end{array}$ & CRD Identifier & County Name & County FIPS & $\begin{array}{c}\text { USDA Farm Production } \\
\text { Region }\end{array}$ \\
\hline Idaho & Idaho CRD 10 & 16010 & Lewis & 16061 & Mountain \\
\hline Idaho & Idaho CRD 80 & 16080 & Lincoln & 16063 & Mountain \\
\hline Idaho & Idaho CRD 90 & 16090 & Madison & 16065 & Mountain \\
\hline Idaho & Idaho CRD 80 & 16080 & Minidoka & 16067 & Mountain \\
\hline Idaho & Idaho CRD 10 & 16010 & Nez Perce & 16069 & Mountain \\
\hline Idaho & Idaho CRD 90 & 16090 & Oneida & 16071 & Mountain \\
\hline Idaho & Idaho CRD 70 & 16070 & Owyhee & 16073 & Mountain \\
\hline Idaho & Idaho CRD 70 & 16070 & Payette & 16075 & Mountain \\
\hline Idaho & Idaho CRD 90 & 16090 & Power & 16077 & Mountain \\
\hline Idaho & Idaho CRD 10 & 16010 & Shoshone & 16079 & Mountain \\
\hline Idaho & Idaho CRD 90 & 16090 & Teton & 16081 & Mountain \\
\hline Idaho & Idaho CRD 80 & 16080 & Twin Falls & 16083 & Mountain \\
\hline Idaho & Idaho CRD 70 & 16070 & Valley & 16085 & Mountain \\
\hline Idaho & Idaho CRD 70 & 16070 & Washington & 16087 & Mountain \\
\hline Illinois & Illinois CRD 30 & 17030 & Adams & 17001 & Corn-belt \\
\hline Illinois & Illinois CRD 80 & 17080 & Alexander & 17003 & Corn-belt \\
\hline Illinois & Illinois CRD 60 & 17060 & Bond & 17005 & Corn-belt \\
\hline Illinois & Illinois CRD 20 & 17020 & Boone & 17007 & Corn-belt \\
\hline Illinois & Illinois CRD 30 & 17030 & Brown & 17009 & Corn-belt \\
\hline Illinois & Illinois CRD 10 & 17010 & Bureau & 17011 & Corn-belt \\
\hline Illinois & Illinois CRD 60 & 17060 & Calhoun & 17013 & Corn-belt \\
\hline Illinois & Illinois CRD 10 & 17010 & Carroll & 17015 & Corn-belt \\
\hline Illinois & Illinois CRD 60 & 17060 & Cass & 17017 & Corn-belt \\
\hline Illinois & Illinois CRD 50 & 17050 & Champaign & 17019 & Corn-belt \\
\hline Illinois & Illinois CRD 60 & 17060 & Christian & 17021 & Corn-belt \\
\hline Illinois & Illinois CRD 70 & 17070 & Clark & 17023 & Corn-belt \\
\hline Illinois & Illinois CRD 70 & 17070 & Clay & 17025 & Corn-belt \\
\hline Illinois & Illinois CRD 80 & 17080 & Clinton & 17027 & Corn-belt \\
\hline Illinois & Illinois CRD 70 & 17070 & Coles & 17029 & Corn-belt \\
\hline Illinois & Illinois CRD 20 & 17020 & Cook & 17031 & Corn-belt \\
\hline Illinois & Illinois CRD 70 & 17070 & Crawford & 17033 & Corn-belt \\
\hline Illinois & Illinois CRD 70 & 17070 & Cumberland & 17035 & Corn-belt \\
\hline Illinois & Illinois CRD 20 & 17020 & De Kalb & 17037 & Corn-belt \\
\hline Illinois & Illinois CRD 40 & 17040 & De Witt & 17039 & Corn-belt \\
\hline Illinois & Illinois CRD 70 & 17070 & Douglas & 17041 & Corn-belt \\
\hline Illinois & Illinois CRD 20 & 17020 & Du Page & 17043 & Corn-belt \\
\hline Illinois & Illinois CRD 70 & 17070 & Edgar & 17045 & Corn-belt \\
\hline Illinois & Illinois CRD 90 & 17090 & Edwards & 17047 & Corn-belt \\
\hline Illinois & Illinois CRD 70 & 17070 & Effingham & 17049 & Corn-belt \\
\hline Illinois & Illinois CRD 70 & 17070 & Fayette & 17051 & Corn-belt \\
\hline Illinois & Illinois CRD 50 & 17050 & Ford & 17053 & Corn-belt \\
\hline Illinois & Illinois CRD 90 & 17090 & Franklin & 17055 & Corn-belt \\
\hline
\end{tabular}


Table 1. Crop Reporting Districts (CRD) of the conterminous United States and associated counties.-Continued

[FIPS, Federal Information Processing Standard; USDA, U.S. Department of Agriculture]

\begin{tabular}{|c|c|c|c|c|c|}
\hline State & $\begin{array}{l}\text { Crop Reporting District } \\
\text { (CRD) }\end{array}$ & CRD Identifier & County Name & County FIPS & $\begin{array}{c}\text { USDA Farm Production } \\
\text { Region }\end{array}$ \\
\hline Illinois & Illinois CRD 30 & 17030 & Fulton & 17057 & Corn-belt \\
\hline Illinois & Illinois CRD 90 & 17090 & Gallatin & 17059 & Corn-belt \\
\hline Illinois & Illinois CRD 60 & 17060 & Greene & 17061 & Corn-belt \\
\hline Illinois & Illinois CRD 20 & 17020 & Grundy & 17063 & Corn-belt \\
\hline Illinois & Illinois CRD 90 & 17090 & Hamilton & 17065 & Corn-belt \\
\hline Illinois & Illinois CRD 30 & 17030 & Hancock & 17067 & Corn-belt \\
\hline Illinois & Illinois CRD 90 & 17090 & Hardin & 17069 & Corn-belt \\
\hline Illinois & Illinois CRD 30 & 17030 & Henderson & 17071 & Corn-belt \\
\hline Illinois & Illinois CRD 10 & 17010 & Henry & 17073 & Corn-belt \\
\hline Illinois & Illinois CRD 50 & 17050 & Iroquois & 17075 & Corn-belt \\
\hline Illinois & Illinois CRD 80 & 17080 & Jackson & 17077 & Corn-belt \\
\hline Illinois & Illinois CRD 70 & 17070 & Jasper & 17079 & Corn-belt \\
\hline Illinois & Illinois CRD 90 & 17090 & Jefferson & 17081 & Corn-belt \\
\hline Illinois & Illinois CRD 60 & 17060 & Jersey & 17083 & Corn-belt \\
\hline Illinois & Illinois CRD 10 & 17010 & Jo Daviess & 17085 & Corn-belt \\
\hline Illinois & Illinois CRD 80 & 17080 & Johnson & 17087 & Corn-belt \\
\hline Illinois & Illinois CRD 20 & 17020 & Kane & 17089 & Corn-belt \\
\hline Illinois & Illinois CRD 50 & 17050 & Kankakee & 17091 & Corn-belt \\
\hline Illinois & Illinois CRD 20 & 17020 & Kendall & 17093 & Corn-belt \\
\hline Illinois & Illinois CRD 30 & 17030 & Knox & 17095 & Corn-belt \\
\hline Illinois & Illinois CRD 20 & 17020 & Lake & 17097 & Corn-belt \\
\hline Illinois & Illinois CRD 20 & 17020 & La Salle & 17099 & Corn-belt \\
\hline Illinois & Illinois CRD 70 & 17070 & Lawrence & 17101 & Corn-belt \\
\hline Illinois & Illinois CRD 10 & 17010 & Lee & 17103 & Corn-belt \\
\hline Illinois & Illinois CRD 50 & 17050 & Livingston & 17105 & Corn-belt \\
\hline Illinois & Illinois CRD 40 & 17040 & Logan & 17107 & Corn-belt \\
\hline Illinois & Illinois CRD 30 & 17030 & McDonough & 17109 & Corn-belt \\
\hline Illinois & Illinois CRD 20 & 17020 & McHenry & 17111 & Corn-belt \\
\hline Illinois & Illinois CRD 40 & 17040 & McLean & 17113 & Corn-belt \\
\hline Illinois & Illinois CRD 40 & 17040 & Macon & 17115 & Corn-belt \\
\hline Illinois & Illinois CRD 60 & 17060 & Macoupin & 17117 & Corn-belt \\
\hline Illinois & Illinois CRD 60 & 17060 & Madison & 17119 & Corn-belt \\
\hline Illinois & Illinois CRD 70 & 17070 & Marion & 17121 & Corn-belt \\
\hline Illinois & Illinois CRD 40 & 17040 & Marshall & 17123 & Corn-belt \\
\hline Illinois & Illinois CRD 40 & 17040 & Mason & 17125 & Corn-belt \\
\hline Illinois & Illinois CRD 90 & 17090 & Massac & 17127 & Corn-belt \\
\hline Illinois & Illinois CRD 40 & 17040 & Menard & 17129 & Corn-belt \\
\hline Illinois & Illinois CRD 10 & 17010 & Mercer & 17131 & Corn-belt \\
\hline Illinois & Illinois CRD 80 & 17080 & Monroe & 17133 & Corn-belt \\
\hline Illinois & Illinois CRD 60 & 17060 & Montgomery & 17135 & Corn-belt \\
\hline Illinois & Illinois CRD 60 & 17060 & Morgan & 17137 & Corn-belt \\
\hline Illinois & Illinois CRD 70 & 17070 & Moultrie & 17139 & Corn-belt \\
\hline
\end{tabular}


Table 1. Crop Reporting Districts (CRD) of the conterminous United States and associated counties.-Continued

[FIPS, Federal Information Processing Standard; USDA, U.S. Department of Agriculture]

\begin{tabular}{|c|c|c|c|c|c|}
\hline State & $\begin{array}{l}\text { Crop Reporting District } \\
\text { (CRD) }\end{array}$ & CRD Identifier & County Name & County FIPS & $\begin{array}{c}\text { USDA Farm Production } \\
\text { Region }\end{array}$ \\
\hline Illinois & Illinois CRD 10 & 17010 & Ogle & 17141 & Corn-belt \\
\hline Illinois & Illinois CRD 40 & 17040 & Peoria & 17143 & Corn-belt \\
\hline Illinois & Illinois CRD 80 & 17080 & Perry & 17145 & Corn-belt \\
\hline Illinois & Illinois CRD 50 & 17050 & Piatt & 17147 & Corn-belt \\
\hline Illinois & Illinois CRD 60 & 17060 & Pike & 17149 & Corn-belt \\
\hline Illinois & Illinois CRD 90 & 17090 & Pope & 17151 & Corn-belt \\
\hline Illinois & Illinois CRD 80 & 17080 & Pulaski & 17153 & Corn-belt \\
\hline Illinois & Illinois CRD 10 & 17010 & Putnam & 17155 & Corn-belt \\
\hline Illinois & Illinois CRD 80 & 17080 & Randolph & 17157 & Corn-belt \\
\hline Illinois & Illinois CRD 70 & 17070 & Richland & 17159 & Corn-belt \\
\hline Illinois & Illinois CRD 10 & 17010 & Rock Island & 17161 & Corn-belt \\
\hline Illinois & Illinois CRD 80 & 17080 & St. Clair & 17163 & Corn-belt \\
\hline Illinois & Illinois CRD 90 & 17090 & Saline & 17165 & Corn-belt \\
\hline Illinois & Illinois CRD 60 & 17060 & Sangamon & 17167 & Corn-belt \\
\hline Illinois & Illinois CRD 30 & 17030 & Schuyler & 17169 & Corn-belt \\
\hline Illinois & Illinois CRD 60 & 17060 & Scott & 17171 & Corn-belt \\
\hline Illinois & Illinois CRD 70 & 17070 & Shelby & 17173 & Corn-belt \\
\hline Illinois & Illinois CRD 40 & 17040 & Stark & 17175 & Corn-belt \\
\hline Illinois & Illinois CRD 10 & 17010 & Stephenson & 17177 & Corn-belt \\
\hline Illinois & Illinois CRD 40 & 17040 & Tazewell & 17179 & Corn-belt \\
\hline Illinois & Illinois CRD 80 & 17080 & Union & 17181 & Corn-belt \\
\hline Illinois & Illinois CRD 50 & 17050 & Vermilion & 17183 & Corn-belt \\
\hline Illinois & Illinois CRD 90 & 17090 & Wabash & 17185 & Corn-belt \\
\hline Illinois & Illinois CRD 30 & 17030 & Warren & 17187 & Corn-belt \\
\hline Illinois & Illinois CRD 80 & 17080 & Washington & 17189 & Corn-belt \\
\hline Illinois & Illinois CRD 90 & 17090 & Wayne & 17191 & Corn-belt \\
\hline Illinois & Illinois CRD 90 & 17090 & White & 17193 & Corn-belt \\
\hline Illinois & Illinois CRD 10 & 17010 & Whiteside & 17195 & Corn-belt \\
\hline Illinois & Illinois CRD 20 & 17020 & Will & 17197 & Corn-belt \\
\hline Illinois & Illinois CRD 80 & 17080 & Williamson & 17199 & Corn-belt \\
\hline Illinois & Illinois CRD 10 & 17010 & Winnebago & 17201 & Corn-belt \\
\hline Illinois & Illinois CRD 40 & 17040 & Woodford & 17203 & Corn-belt \\
\hline Indiana & Indiana CRD 30 & 18030 & Adams & 18001 & Corn-belt \\
\hline Indiana & Indiana CRD 30 & 18030 & Allen & 18003 & Corn-belt \\
\hline Indiana & Indiana CRD 50 & 18050 & Bartholomew & 18005 & Corn-belt \\
\hline Indiana & Indiana CRD 10 & 18010 & Benton & 18007 & Corn-belt \\
\hline Indiana & Indiana CRD 60 & 18060 & Blackford & 18009 & Corn-belt \\
\hline Indiana & Indiana CRD 50 & 18050 & Boone & 18011 & Corn-belt \\
\hline Indiana & Indiana CRD 80 & 18080 & Brown & 18013 & Corn-belt \\
\hline Indiana & Indiana CRD 20 & 18020 & Carroll & 18015 & Corn-belt \\
\hline Indiana & Indiana CRD 20 & 18020 & Cass & 18017 & Corn-belt \\
\hline Indiana & Indiana CRD 90 & 18090 & Clark & 18019 & Corn-belt \\
\hline
\end{tabular}


Table 1. Crop Reporting Districts (CRD) of the conterminous United States and associated counties.-Continued

[FIPS, Federal Information Processing Standard; USDA, U.S. Department of Agriculture]

\begin{tabular}{|c|c|c|c|c|c|}
\hline State & $\begin{array}{l}\text { Crop Reporting District } \\
\text { (CRD) }\end{array}$ & CRD Identifier & County Name & County FIPS & $\begin{array}{c}\text { USDA Farm Production } \\
\text { Region }\end{array}$ \\
\hline Indiana & Indiana CRD 40 & 18040 & Clay & 18021 & Corn-belt \\
\hline Indiana & Indiana CRD 50 & 18050 & Clinton & 18023 & Corn-belt \\
\hline Indiana & Indiana CRD 80 & 18080 & Crawford & 18025 & Corn-belt \\
\hline Indiana & Indiana CRD 70 & 18070 & Daviess & 18027 & Corn-belt \\
\hline Indiana & Indiana CRD 90 & 18090 & Dearborn & 18029 & Corn-belt \\
\hline Indiana & Indiana CRD 50 & 18050 & Decatur & 18031 & Corn-belt \\
\hline Indiana & Indiana CRD 30 & 18030 & De Kalb & 18033 & Corn-belt \\
\hline Indiana & Indiana CRD 60 & 18060 & Delaware & 18035 & Corn-belt \\
\hline Indiana & Indiana CRD 70 & 18070 & Dubois & 18037 & Corn-belt \\
\hline Indiana & Indiana CRD 20 & 18020 & Elkhart & 18039 & Corn-belt \\
\hline Indiana & Indiana CRD 60 & 18060 & Fayette & 18041 & Corn-belt \\
\hline Indiana & Indiana CRD 80 & 18080 & Floyd & 18043 & Corn-belt \\
\hline Indiana & Indiana CRD 40 & 18040 & Fountain & 18045 & Corn-belt \\
\hline Indiana & Indiana CRD 90 & 18090 & Franklin & 18047 & Corn-belt \\
\hline Indiana & Indiana CRD 20 & 18020 & Fulton & 18049 & Corn-belt \\
\hline Indiana & Indiana CRD 70 & 18070 & Gibson & 18051 & Corn-belt \\
\hline Indiana & Indiana CRD 50 & 18050 & Grant & 18053 & Corn-belt \\
\hline Indiana & Indiana CRD 70 & 18070 & Greene & 18055 & Corn-belt \\
\hline Indiana & Indiana CRD 50 & 18050 & Hamilton & 18057 & Corn-belt \\
\hline Indiana & Indiana CRD 50 & 18050 & Hancock & 18059 & Corn-belt \\
\hline Indiana & Indiana CRD 80 & 18080 & Harrison & 18061 & Corn-belt \\
\hline Indiana & Indiana CRD 50 & 18050 & Hendricks & 18063 & Corn-belt \\
\hline Indiana & Indiana CRD 60 & 18060 & Henry & 18065 & Corn-belt \\
\hline Indiana & Indiana CRD 50 & 18050 & Howard & 18067 & Corn-belt \\
\hline Indiana & Indiana CRD 30 & 18030 & Huntington & 18069 & Corn-belt \\
\hline Indiana & Indiana CRD 80 & 18080 & Jackson & 18071 & Corn-belt \\
\hline Indiana & Indiana CRD 10 & 18010 & Jasper & 18073 & Corn-belt \\
\hline Indiana & Indiana CRD 60 & 18060 & Jay & 18075 & Corn-belt \\
\hline Indiana & Indiana CRD 90 & 18090 & Jefferson & 18077 & Corn-belt \\
\hline Indiana & Indiana CRD 90 & 18090 & Jennings & 18079 & Corn-belt \\
\hline Indiana & Indiana CRD 50 & 18050 & Johnson & 18081 & Corn-belt \\
\hline Indiana & Indiana CRD 70 & 18070 & Knox & 18083 & Corn-belt \\
\hline Indiana & Indiana CRD 20 & 18020 & Kosciusko & 18085 & Corn-belt \\
\hline Indiana & Indiana CRD 30 & 18030 & Lagrange & 18087 & Corn-belt \\
\hline Indiana & Indiana CRD 10 & 18010 & Lake & 18089 & Corn-belt \\
\hline Indiana & Indiana CRD 10 & 18010 & La Porte & 18091 & Corn-belt \\
\hline Indiana & Indiana CRD 80 & 18080 & Lawrence & 18093 & Corn-belt \\
\hline Indiana & Indiana CRD 50 & 18050 & Madison & 18095 & Corn-belt \\
\hline Indiana & Indiana CRD 50 & 18050 & Marion & 18097 & Corn-belt \\
\hline Indiana & Indiana CRD 20 & 18020 & Marshall & 18099 & Corn-belt \\
\hline Indiana & Indiana CRD 70 & 18070 & Martin & 18101 & Corn-belt \\
\hline Indiana & Indiana CRD 20 & 18020 & Miami & 18103 & Corn-belt \\
\hline
\end{tabular}


Table 1. Crop Reporting Districts (CRD) of the conterminous United States and associated counties.-Continued

[FIPS, Federal Information Processing Standard; USDA, U.S. Department of Agriculture]

\begin{tabular}{|c|c|c|c|c|c|}
\hline State & $\begin{array}{l}\text { Crop Reporting District } \\
\text { (CRD) }\end{array}$ & CRD Identifier & County Name & County FIPS & $\begin{array}{c}\text { USDA Farm Production } \\
\text { Region }\end{array}$ \\
\hline Indiana & Indiana CRD 80 & 18080 & Monroe & 18105 & Corn-belt \\
\hline Indiana & Indiana CRD 40 & 18040 & Montgomery & 18107 & Corn-belt \\
\hline Indiana & Indiana CRD 50 & 18050 & Morgan & 18109 & Corn-belt \\
\hline Indiana & Indiana CRD 10 & 18010 & Newton & 18111 & Corn-belt \\
\hline Indiana & Indiana CRD 30 & 18030 & Noble & 18113 & Corn-belt \\
\hline Indiana & Indiana CRD 90 & 18090 & Ohio & 18115 & Corn-belt \\
\hline Indiana & Indiana CRD 80 & 18080 & Orange & 18117 & Corn-belt \\
\hline Indiana & Indiana CRD 40 & 18040 & Owen & 18119 & Corn-belt \\
\hline Indiana & Indiana CRD 40 & 18040 & Parke & 18121 & Corn-belt \\
\hline Indiana & Indiana CRD 80 & 18080 & Perry & 18123 & Corn-belt \\
\hline Indiana & Indiana CRD 70 & 18070 & Pike & 18125 & Corn-belt \\
\hline Indiana & Indiana CRD 10 & 18010 & Porter & 18127 & Corn-belt \\
\hline Indiana & Indiana CRD 70 & 18070 & Posey & 18129 & Corn-belt \\
\hline Indiana & Indiana CRD 10 & 18010 & Pulaski & 18131 & Corn-belt \\
\hline Indiana & Indiana CRD 40 & 18040 & Putnam & 18133 & Corn-belt \\
\hline Indiana & Indiana CRD 60 & 18060 & Randolph & 18135 & Corn-belt \\
\hline Indiana & Indiana CRD 90 & 18090 & Ripley & 18137 & Corn-belt \\
\hline Indiana & Indiana CRD 50 & 18050 & Rush & 18139 & Corn-belt \\
\hline Indiana & Indiana CRD 20 & 18020 & St. Joseph & 18141 & Corn-belt \\
\hline Indiana & Indiana CRD 90 & 18090 & Scott & 18143 & Corn-belt \\
\hline Indiana & Indiana CRD 50 & 18050 & Shelby & 18145 & Corn-belt \\
\hline Indiana & Indiana CRD 70 & 18070 & Spencer & 18147 & Corn-belt \\
\hline Indiana & Indiana CRD 10 & 18010 & Starke & 18149 & Corn-belt \\
\hline Indiana & Indiana CRD 30 & 18030 & Steuben & 18151 & Corn-belt \\
\hline Indiana & Indiana CRD 70 & 18070 & Sullivan & 18153 & Corn-belt \\
\hline Indiana & Indiana CRD 90 & 18090 & Switzerland & 18155 & Corn-belt \\
\hline Indiana & Indiana CRD 40 & 18040 & Tippecanoe & 18157 & Corn-belt \\
\hline Indiana & Indiana CRD 50 & 18050 & Tipton & 18159 & Corn-belt \\
\hline Indiana & Indiana CRD 60 & 18060 & Union & 18161 & Corn-belt \\
\hline Indiana & Indiana CRD 70 & 18070 & Vanderburgh & 18163 & Corn-belt \\
\hline Indiana & Indiana CRD 40 & 18040 & Vermillion & 18165 & Corn-belt \\
\hline Indiana & Indiana CRD 40 & 18040 & Vigo & 18167 & Corn-belt \\
\hline Indiana & Indiana CRD 20 & 18020 & Wabash & 18169 & Corn-belt \\
\hline Indiana & Indiana CRD 40 & 18040 & Warren & 18171 & Corn-belt \\
\hline Indiana & Indiana CRD 70 & 18070 & Warrick & 18173 & Corn-belt \\
\hline Indiana & Indiana CRD 80 & 18080 & Washington & 18175 & Corn-belt \\
\hline Indiana & Indiana CRD 60 & 18060 & Wayne & 18177 & Corn-belt \\
\hline Indiana & Indiana CRD 30 & 18030 & Wells & 18179 & Corn-belt \\
\hline Indiana & Indiana CRD 10 & 18010 & White & 18181 & Corn-belt \\
\hline Indiana & Indiana CRD 30 & 18030 & Whitley & 18183 & Corn-belt \\
\hline Iowa & Iowa CRD 70 & 19070 & Adair & 19001 & Corn-belt \\
\hline Iowa & Iowa CRD 70 & 19070 & Adams & 19003 & Corn-belt \\
\hline
\end{tabular}


Table 1. Crop Reporting Districts (CRD) of the conterminous United States and associated counties.—Continued

[FIPS, Federal Information Processing Standard; USDA, U.S. Department of Agriculture]

\begin{tabular}{|c|c|c|c|c|c|}
\hline State & $\begin{array}{l}\text { Crop Reporting District } \\
\text { (CRD) }\end{array}$ & CRD Identifier & County Name & County FIPS & $\begin{array}{c}\text { USDA Farm Production } \\
\text { Region }\end{array}$ \\
\hline Iowa & Iowa CRD 30 & 19030 & Allamakee & 19005 & Corn-belt \\
\hline Iowa & Iowa CRD 80 & 19080 & Appanoose & 19007 & Corn-belt \\
\hline Iowa & Iowa CRD 40 & 19040 & Audubon & 19009 & Corn-belt \\
\hline Iowa & Iowa CRD 60 & 19060 & Benton & 19011 & Corn-belt \\
\hline Iowa & Iowa CRD 30 & 19030 & Black Hawk & 19013 & Corn-belt \\
\hline Iowa & Iowa CRD 50 & 19050 & Boone & 19015 & Corn-belt \\
\hline Iowa & Iowa CRD 30 & 19030 & Bremer & 19017 & Corn-belt \\
\hline Iowa & Iowa CRD 30 & 19030 & Buchanan & 19019 & Corn-belt \\
\hline Iowa & Iowa CRD 10 & 19010 & Buena Vista & 19021 & Corn-belt \\
\hline Iowa & Iowa CRD 20 & 19020 & Butler & 19023 & Corn-belt \\
\hline Iowa & Iowa CRD 40 & 19040 & Calhoun & 19025 & Corn-belt \\
\hline Iowa & Iowa CRD 40 & 19040 & Carroll & 19027 & Corn-belt \\
\hline Iowa & Iowa CRD 70 & 19070 & Cass & 19029 & Corn-belt \\
\hline Iowa & Iowa CRD 60 & 19060 & Cedar & 19031 & Corn-belt \\
\hline Iowa & Iowa CRD 20 & 19020 & Cerro Gordo & 19033 & Corn-belt \\
\hline Iowa & Iowa CRD 10 & 19010 & Cherokee & 19035 & Corn-belt \\
\hline Iowa & Iowa CRD 30 & 19030 & Chickasaw & 19037 & Corn-belt \\
\hline Iowa & Iowa CRD 80 & 19080 & Clarke & 19039 & Corn-belt \\
\hline Iowa & Iowa CRD 10 & 19010 & Clay & 19041 & Corn-belt \\
\hline Iowa & Iowa CRD 30 & 19030 & Clayton & 19043 & Corn-belt \\
\hline Iowa & Iowa CRD 60 & 19060 & Clinton & 19045 & Corn-belt \\
\hline Iowa & Iowa CRD 40 & 19040 & Crawford & 19047 & Corn-belt \\
\hline Iowa & Iowa CRD 50 & 19050 & Dallas & 19049 & Corn-belt \\
\hline Iowa & Iowa CRD 90 & 19090 & Davis & 19051 & Corn-belt \\
\hline Iowa & Iowa CRD 80 & 19080 & Decatur & 19053 & Corn-belt \\
\hline Iowa & Iowa CRD 30 & 19030 & Delaware & 19055 & Corn-belt \\
\hline Iowa & Iowa CRD 90 & 19090 & Des Moines & 19057 & Corn-belt \\
\hline Iowa & Iowa CRD 10 & 19010 & Dickinson & 19059 & Corn-belt \\
\hline Iowa & Iowa CRD 30 & 19030 & Dubuque & 19061 & Corn-belt \\
\hline Iowa & Iowa CRD 10 & 19010 & Emmet & 19063 & Corn-belt \\
\hline Iowa & Iowa CRD 30 & 19030 & Fayette & 19065 & Corn-belt \\
\hline Iowa & Iowa CRD 20 & 19020 & Floyd & 19067 & Corn-belt \\
\hline Iowa & Iowa CRD 20 & 19020 & Franklin & 19069 & Corn-belt \\
\hline Iowa & Iowa CRD 70 & 19070 & Fremont & 19071 & Corn-belt \\
\hline Iowa & Iowa CRD 40 & 19040 & Greene & 19073 & Corn-belt \\
\hline Iowa & Iowa CRD 50 & 19050 & Grundy & 19075 & Corn-belt \\
\hline Iowa & Iowa CRD 40 & 19040 & Guthrie & 19077 & Corn-belt \\
\hline Iowa & Iowa CRD 50 & 19050 & Hamilton & 19079 & Corn-belt \\
\hline Iowa & Iowa CRD 20 & 19020 & Hancock & 19081 & Corn-belt \\
\hline Iowa & Iowa CRD 50 & 19050 & Hardin & 19083 & Corn-belt \\
\hline Iowa & Iowa CRD 40 & 19040 & Harrison & 19085 & Corn-belt \\
\hline Iowa & Iowa CRD 90 & 19090 & Henry & 19087 & Corn-belt \\
\hline
\end{tabular}


Table 1. Crop Reporting Districts (CRD) of the conterminous United States and associated counties.-Continued

[FIPS, Federal Information Processing Standard; USDA, U.S. Department of Agriculture]

\begin{tabular}{|c|c|c|c|c|c|}
\hline State & $\begin{array}{l}\text { Crop Reporting District } \\
\text { (CRD) }\end{array}$ & CRD Identifier & County Name & County FIPS & $\begin{array}{c}\text { USDA Farm Production } \\
\text { Region }\end{array}$ \\
\hline Iowa & Iowa CRD 30 & 19030 & Howard & 19089 & Corn-belt \\
\hline Iowa & Iowa CRD 20 & 19020 & Humboldt & 19091 & Corn-belt \\
\hline Iowa & Iowa CRD 40 & 19040 & Ida & 19093 & Corn-belt \\
\hline Iowa & Iowa CRD 60 & 19060 & Iowa & 19095 & Corn-belt \\
\hline Iowa & Iowa CRD 60 & 19060 & Jackson & 19097 & Corn-belt \\
\hline Iowa & Iowa CRD 50 & 19050 & Jasper & 19099 & Corn-belt \\
\hline Iowa & Iowa CRD 90 & 19090 & Jefferson & 19101 & Corn-belt \\
\hline Iowa & Iowa CRD 60 & 19060 & Johnson & 19103 & Corn-belt \\
\hline Iowa & Iowa CRD 60 & 19060 & Jones & 19105 & Corn-belt \\
\hline Iowa & Iowa CRD 90 & 19090 & Keokuk & 19107 & Corn-belt \\
\hline Iowa & Iowa CRD 20 & 19020 & Kossuth & 19109 & Corn-belt \\
\hline Iowa & Iowa CRD 90 & 19090 & Lee & 19111 & Corn-belt \\
\hline Iowa & Iowa CRD 60 & 19060 & Linn & 19113 & Corn-belt \\
\hline Iowa & Iowa CRD 90 & 19090 & Louisa & 19115 & Corn-belt \\
\hline Iowa & Iowa CRD 80 & 19080 & Lucas & 19117 & Corn-belt \\
\hline Iowa & Iowa CRD 10 & 19010 & Lyon & 19119 & Corn-belt \\
\hline Iowa & Iowa CRD 80 & 19080 & Madison & 19121 & Corn-belt \\
\hline Iowa & Iowa CRD 90 & 19090 & Mahaska & 19123 & Corn-belt \\
\hline Iowa & Iowa CRD 80 & 19080 & Marion & 19125 & Corn-belt \\
\hline Iowa & Iowa CRD 50 & 19050 & Marshall & 19127 & Corn-belt \\
\hline Iowa & Iowa CRD 70 & 19070 & Mills & 19129 & Corn-belt \\
\hline Iowa & Iowa CRD 20 & 19020 & Mitchell & 19131 & Corn-belt \\
\hline Iowa & Iowa CRD 40 & 19040 & Monona & 19133 & Corn-belt \\
\hline Iowa & Iowa CRD 80 & 19080 & Monroe & 19135 & Corn-belt \\
\hline Iowa & Iowa CRD 70 & 19070 & Montgomery & 19137 & Corn-belt \\
\hline Iowa & Iowa CRD 60 & 19060 & Muscatine & 19139 & Corn-belt \\
\hline Iowa & Iowa CRD 10 & 19010 & O'Brien & 19141 & Corn-belt \\
\hline Iowa & Iowa CRD 10 & 19010 & Osceola & 19143 & Corn-belt \\
\hline Iowa & Iowa CRD 70 & 19070 & Page & 19145 & Corn-belt \\
\hline Iowa & Iowa CRD 10 & 19010 & Palo Alto & 19147 & Corn-belt \\
\hline Iowa & Iowa CRD 10 & 19010 & Plymouth & 19149 & Corn-belt \\
\hline Iowa & Iowa CRD 10 & 19010 & Pocahontas & 19151 & Corn-belt \\
\hline Iowa & Iowa CRD 50 & 19050 & Polk & 19153 & Corn-belt \\
\hline Iowa & Iowa CRD 70 & 19070 & Pottawattamie & 19155 & Corn-belt \\
\hline Iowa & Iowa CRD 50 & 19050 & Poweshiek & 19157 & Corn-belt \\
\hline Iowa & Iowa CRD 80 & 19080 & Ringgold & 19159 & Corn-belt \\
\hline Iowa & Iowa CRD 40 & 19040 & $\mathrm{Sac}$ & 19161 & Corn-belt \\
\hline Iowa & Iowa CRD 60 & 19060 & Scott & 19163 & Corn-belt \\
\hline Iowa & Iowa CRD 40 & 19040 & Shelby & 19165 & Corn-belt \\
\hline Iowa & Iowa CRD 10 & 19010 & Sioux & 19167 & Corn-belt \\
\hline Iowa & Iowa CRD 50 & 19050 & Story & 19169 & Corn-belt \\
\hline Iowa & Iowa CRD 50 & 19050 & Tama & 19171 & Corn-belt \\
\hline
\end{tabular}


Table 1. Crop Reporting Districts (CRD) of the conterminous United States and associated counties.—Continued

[FIPS, Federal Information Processing Standard; USDA, U.S. Department of Agriculture]

\begin{tabular}{|c|c|c|c|c|c|}
\hline State & $\begin{array}{l}\text { Crop Reporting District } \\
\text { (CRD) }\end{array}$ & CRD Identifier & County Name & County FIPS & $\begin{array}{l}\text { USDA Farm Production } \\
\text { Region }\end{array}$ \\
\hline Iowa & Iowa CRD 70 & 19070 & Taylor & 19173 & Corn-belt \\
\hline Iowa & Iowa CRD 80 & 19080 & Union & 19175 & Corn-belt \\
\hline Iowa & Iowa CRD 90 & 19090 & Van Buren & 19177 & Corn-belt \\
\hline Iowa & Iowa CRD 90 & 19090 & Wapello & 19179 & Corn-belt \\
\hline Iowa & Iowa CRD 80 & 19080 & Warren & 19181 & Corn-belt \\
\hline Iowa & Iowa CRD 90 & 19090 & Washington & 19183 & Corn-belt \\
\hline Iowa & Iowa CRD 80 & 19080 & Wayne & 19185 & Corn-belt \\
\hline Iowa & Iowa CRD 50 & 19050 & Webster & 19187 & Corn-belt \\
\hline Iowa & Iowa CRD 20 & 19020 & Winnebago & 19189 & Corn-belt \\
\hline Iowa & Iowa CRD 30 & 19030 & Winneshiek & 19191 & Corn-belt \\
\hline Iowa & Iowa CRD 40 & 19040 & Woodbury & 19193 & Corn-belt \\
\hline Iowa & Iowa CRD 20 & 19020 & Worth & 19195 & Corn-belt \\
\hline Iowa & Iowa CRD 20 & 19020 & Wright & 19197 & Corn-belt \\
\hline Kansas & Kansas CRD 90 & 20090 & Allen & 20001 & Northern-plains \\
\hline Kansas & Kansas CRD 80 & 20080 & Anderson & 20003 & Northern-plains \\
\hline Kansas & Kansas CRD 70 & 20070 & Atchison & 20005 & Northern-plains \\
\hline Kansas & Kansas CRD 60 & 20060 & Barber & 20007 & Northern-plains \\
\hline Kansas & Kansas CRD 50 & 20050 & Barton & 20009 & Northern-plains \\
\hline Kansas & Kansas CRD 90 & 20090 & Bourbon & 20011 & Northern-plains \\
\hline Kansas & Kansas CRD 70 & 20070 & Brown & 20013 & Northern-plains \\
\hline Kansas & Kansas CRD 90 & 20090 & Butler & 20015 & Northern-plains \\
\hline Kansas & Kansas CRD 80 & 20080 & Chase & 20017 & Northern-plains \\
\hline Kansas & Kansas CRD 90 & 20090 & Chautauqua & 20019 & Northern-plains \\
\hline Kansas & Kansas CRD 90 & 20090 & Cherokee & 20021 & Northern-plains \\
\hline Kansas & Kansas CRD 10 & 20010 & Cheyenne & 20023 & Northern-plains \\
\hline Kansas & Kansas CRD 30 & 20030 & Clark & 20025 & Northern-plains \\
\hline Kansas & Kansas CRD 40 & 20040 & Clay & 20027 & Northern-plains \\
\hline Kansas & Kansas CRD 40 & 20040 & Cloud & 20029 & Northern-plains \\
\hline Kansas & Kansas CRD 80 & 20080 & Coffey & 20031 & Northern-plains \\
\hline Kansas & Kansas CRD 60 & 20060 & Comanche & 20033 & Northern-plains \\
\hline Kansas & Kansas CRD 90 & 20090 & Cowley & 20035 & Northern-plains \\
\hline Kansas & Kansas CRD 90 & 20090 & Crawford & 20037 & Northern-plains \\
\hline Kansas & Kansas CRD 10 & 20010 & Decatur & 20039 & Northern-plains \\
\hline Kansas & Kansas CRD 50 & 20050 & Dickinson & 20041 & Northern-plains \\
\hline Kansas & Kansas CRD 70 & 20070 & Doniphan & 20043 & Northern-plains \\
\hline Kansas & Kansas CRD 80 & 20080 & Douglas & 20045 & Northern-plains \\
\hline Kansas & Kansas CRD 60 & 20060 & Edwards & 20047 & Northern-plains \\
\hline Kansas & Kansas CRD 90 & 20090 & Elk & 20049 & Northern-plains \\
\hline Kansas & Kansas CRD 50 & 20050 & Ellis & 20051 & Northern-plains \\
\hline Kansas & Kansas CRD 50 & 20050 & Ellsworth & 20053 & Northern-plains \\
\hline Kansas & Kansas CRD 30 & 20030 & Finney & 20055 & Northern-plains \\
\hline Kansas & Kansas CRD 30 & 20030 & Ford & 20057 & Northern-plains \\
\hline
\end{tabular}


Table 1. Crop Reporting Districts (CRD) of the conterminous United States and associated counties. - Continued

[FIPS, Federal Information Processing Standard; USDA, U.S. Department of Agriculture]

\begin{tabular}{|c|c|c|c|c|c|}
\hline State & $\begin{array}{l}\text { Crop Reporting District } \\
\text { (CRD) }\end{array}$ & CRD Identifier & County Name & County FIPS & $\begin{array}{c}\text { USDA Farm Production } \\
\text { Region }\end{array}$ \\
\hline Kansas & Kansas CRD 80 & 20080 & Franklin & 20059 & Northern-plains \\
\hline Kansas & Kansas CRD 80 & 20080 & Geary & 20061 & Northern-plains \\
\hline Kansas & Kansas CRD 20 & 20020 & Gove & 20063 & Northern-plains \\
\hline Kansas & Kansas CRD 10 & 20010 & Graham & 20065 & Northern-plains \\
\hline Kansas & Kansas CRD 30 & 20030 & Grant & 20067 & Northern-plains \\
\hline Kansas & Kansas CRD 30 & 20030 & Gray & 20069 & Northern-plains \\
\hline Kansas & Kansas CRD 20 & 20020 & Greeley & 20071 & Northern-plains \\
\hline Kansas & Kansas CRD 90 & 20090 & Greenwood & 20073 & Northern-plains \\
\hline Kansas & Kansas CRD 30 & 20030 & Hamilton & 20075 & Northern-plains \\
\hline Kansas & Kansas CRD 60 & 20060 & Harper & 20077 & Northern-plains \\
\hline Kansas & Kansas CRD 60 & 20060 & Harvey & 20079 & Northern-plains \\
\hline Kansas & Kansas CRD 30 & 20030 & Haskell & 20081 & Northern-plains \\
\hline Kansas & Kansas CRD 30 & 20030 & Hodgeman & 20083 & Northern-plains \\
\hline Kansas & Kansas CRD 70 & 20070 & Jackson & 20085 & Northern-plains \\
\hline Kansas & Kansas CRD 70 & 20070 & Jefferson & 20087 & Northern-plains \\
\hline Kansas & Kansas CRD 40 & 20040 & Jewell & 20089 & Northern-plains \\
\hline Kansas & Kansas CRD 80 & 20080 & Johnson & 20091 & Northern-plains \\
\hline Kansas & Kansas CRD 30 & 20030 & Kearny & 20093 & Northern-plains \\
\hline Kansas & Kansas CRD 60 & 20060 & Kingman & 20095 & Northern-plains \\
\hline Kansas & Kansas CRD 60 & 20060 & Kiowa & 20097 & Northern-plains \\
\hline Kansas & Kansas CRD 90 & 20090 & Labette & 20099 & Northern-plains \\
\hline Kansas & Kansas CRD 20 & 20020 & Lane & 20101 & Northern-plains \\
\hline Kansas & Kansas CRD 70 & 20070 & Leavenworth & 20103 & Northern-plains \\
\hline Kansas & Kansas CRD 50 & 20050 & Lincoln & 20105 & Northern-plains \\
\hline Kansas & Kansas CRD 80 & 20080 & Linn & 20107 & Northern-plains \\
\hline Kansas & Kansas CRD 20 & 20020 & Logan & 20109 & Northern-plains \\
\hline Kansas & Kansas CRD 80 & 20080 & Lyon & 20111 & Northern-plains \\
\hline Kansas & Kansas CRD 50 & 20050 & McPherson & 20113 & Northern-plains \\
\hline Kansas & Kansas CRD 50 & 20050 & Marion & 20115 & Northern-plains \\
\hline Kansas & Kansas CRD 70 & 20070 & Marshall & 20117 & Northern-plains \\
\hline Kansas & Kansas CRD 30 & 20030 & Meade & 20119 & Northern-plains \\
\hline Kansas & Kansas CRD 80 & 20080 & Miami & 20121 & Northern-plains \\
\hline Kansas & Kansas CRD 40 & 20040 & Mitchell & 20123 & Northern-plains \\
\hline Kansas & Kansas CRD 90 & 20090 & Montgomery & 20125 & Northern-plains \\
\hline Kansas & Kansas CRD 80 & 20080 & Morris & 20127 & Northern-plains \\
\hline Kansas & Kansas CRD 30 & 20030 & Morton & 20129 & Northern-plains \\
\hline Kansas & Kansas CRD 70 & 20070 & Nemaha & 20131 & Northern-plains \\
\hline Kansas & Kansas CRD 90 & 20090 & Neosho & 20133 & Northern-plains \\
\hline Kansas & Kansas CRD 20 & 20020 & Ness & 20135 & Northern-plains \\
\hline Kansas & Kansas CRD 10 & 20010 & Norton & 20137 & Northern-plains \\
\hline Kansas & Kansas CRD 80 & 20080 & Osage & 20139 & Northern-plains \\
\hline Kansas & Kansas CRD 40 & 20040 & Osborne & 20141 & Northern-plains \\
\hline
\end{tabular}


Table 1. Crop Reporting Districts (CRD) of the conterminous United States and associated counties.-Continued

[FIPS, Federal Information Processing Standard; USDA, U.S. Department of Agriculture]

\begin{tabular}{|c|c|c|c|c|c|}
\hline State & $\begin{array}{l}\text { Crop Reporting District } \\
\text { (CRD) }\end{array}$ & CRD Identifier & County Name & County FIPS & $\begin{array}{c}\text { USDA Farm Production } \\
\text { Region }\end{array}$ \\
\hline Kansas & Kansas CRD 40 & 20040 & Ottawa & 20143 & Northern-plains \\
\hline Kansas & Kansas CRD 60 & 20060 & Pawnee & 20145 & Northern-plains \\
\hline Kansas & Kansas CRD 40 & 20040 & Phillips & 20147 & Northern-plains \\
\hline Kansas & Kansas CRD 70 & 20070 & Pottawatomie & 20149 & Northern-plains \\
\hline Kansas & Kansas CRD 60 & 20060 & Pratt & 20151 & Northern-plains \\
\hline Kansas & Kansas CRD 10 & 20010 & Rawlins & 20153 & Northern-plains \\
\hline Kansas & Kansas CRD 60 & 20060 & Reno & 20155 & Northern-plains \\
\hline Kansas & Kansas CRD 40 & 20040 & Republic & 20157 & Northern-plains \\
\hline Kansas & Kansas CRD 50 & 20050 & Rice & 20159 & Northern-plains \\
\hline Kansas & Kansas CRD 70 & 20070 & Riley & 20161 & Northern-plains \\
\hline Kansas & Kansas CRD 40 & 20040 & Rooks & 20163 & Northern-plains \\
\hline Kansas & Kansas CRD 50 & 20050 & Rush & 20165 & Northern-plains \\
\hline Kansas & Kansas CRD 50 & 20050 & Russell & 20167 & Northern-plains \\
\hline Kansas & Kansas CRD 50 & 20050 & Saline & 20169 & Northern-plains \\
\hline Kansas & Kansas CRD 20 & 20020 & Scott & 20171 & Northern-plains \\
\hline Kansas & Kansas CRD 60 & 20060 & Sedgwick & 20173 & Northern-plains \\
\hline Kansas & Kansas CRD 30 & 20030 & Seward & 20175 & Northern-plains \\
\hline Kansas & Kansas CRD 80 & 20080 & Shawnee & 20177 & Northern-plains \\
\hline Kansas & Kansas CRD 10 & 20010 & Sheridan & 20179 & Northern-plains \\
\hline Kansas & Kansas CRD 10 & 20010 & Sherman & 20181 & Northern-plains \\
\hline Kansas & Kansas CRD 40 & 20040 & Smith & 20183 & Northern-plains \\
\hline Kansas & Kansas CRD 60 & 20060 & Stafford & 20185 & Northern-plains \\
\hline Kansas & Kansas CRD 30 & 20030 & Stanton & 20187 & Northern-plains \\
\hline Kansas & Kansas CRD 30 & 20030 & Stevens & 20189 & Northern-plains \\
\hline Kansas & Kansas CRD 60 & 20060 & Sumner & 20191 & Northern-plains \\
\hline Kansas & Kansas CRD 10 & 20010 & Thomas & 20193 & Northern-plains \\
\hline Kansas & Kansas CRD 20 & 20020 & Trego & 20195 & Northern-plains \\
\hline Kansas & Kansas CRD 80 & 20080 & Wabaunsee & 20197 & Northern-plains \\
\hline Kansas & Kansas CRD 20 & 20020 & Wallace & 20199 & Northern-plains \\
\hline Kansas & Kansas CRD 40 & 20040 & Washington & 20201 & Northern-plains \\
\hline Kansas & Kansas CRD 20 & 20020 & Wichita & 20203 & Northern-plains \\
\hline Kansas & Kansas CRD 90 & 20090 & Wilson & 20205 & Northern-plains \\
\hline Kansas & Kansas CRD 90 & 20090 & Woodson & 20207 & Northern-plains \\
\hline Kansas & Kansas CRD 70 & 20070 & Wyandotte & 20209 & Northern-plains \\
\hline Kentucky & Kentucky CRD 30 & 21030 & Adair & 21001 & Appalachian \\
\hline Kentucky & Kentucky CRD 30 & 21030 & Allen & 21003 & Appalachian \\
\hline Kentucky & Kentucky CRD 50 & 21050 & Anderson & 21005 & Appalachian \\
\hline Kentucky & Kentucky CRD 10 & 21010 & Ballard & 21007 & Appalachian \\
\hline Kentucky & Kentucky CRD 30 & 21030 & Barren & 21009 & Appalachian \\
\hline Kentucky & Kentucky CRD 50 & 21050 & Bath & 21011 & Appalachian \\
\hline Kentucky & Kentucky CRD 60 & 21060 & Bell & 21013 & Appalachian \\
\hline Kentucky & Kentucky CRD 40 & 21040 & Boone & 21015 & Appalachian \\
\hline
\end{tabular}


Table 1. Crop Reporting Districts (CRD) of the conterminous United States and associated counties.-Continued

[FIPS, Federal Information Processing Standard; USDA, U.S. Department of Agriculture]

\begin{tabular}{|c|c|c|c|c|c|}
\hline State & $\begin{array}{l}\text { Crop Reporting District } \\
\text { (CRD) }\end{array}$ & CRD Identifier & County Name & County FIPS & $\begin{array}{c}\text { USDA Farm Production } \\
\text { Region }\end{array}$ \\
\hline Kentucky & Kentucky CRD 50 & 21050 & Bourbon & 21017 & Appalachian \\
\hline Kentucky & Kentucky CRD 60 & 21060 & Boyd & 21019 & Appalachian \\
\hline Kentucky & Kentucky CRD 50 & 21050 & Boyle & 21021 & Appalachian \\
\hline Kentucky & Kentucky CRD 40 & 21040 & Bracken & 21023 & Appalachian \\
\hline Kentucky & Kentucky CRD 60 & 21060 & Breathitt & 21025 & Appalachian \\
\hline Kentucky & Kentucky CRD 30 & 21030 & Breckinridge & 21027 & Appalachian \\
\hline Kentucky & Kentucky CRD 30 & 21030 & Bullitt & 21029 & Appalachian \\
\hline Kentucky & Kentucky CRD 30 & 21030 & Butler & 21031 & Appalachian \\
\hline Kentucky & Kentucky CRD 20 & 21020 & Caldwell & 21033 & Appalachian \\
\hline Kentucky & Kentucky CRD 10 & 21010 & Calloway & 21035 & Appalachian \\
\hline Kentucky & Kentucky CRD 40 & 21040 & Campbell & 21037 & Appalachian \\
\hline Kentucky & Kentucky CRD 10 & 21010 & Carlisle & 21039 & Appalachian \\
\hline Kentucky & Kentucky CRD 40 & 21040 & Carroll & 21041 & Appalachian \\
\hline Kentucky & Kentucky CRD 60 & 21060 & Carter & 21043 & Appalachian \\
\hline Kentucky & Kentucky CRD 30 & 21030 & Casey & 21045 & Appalachian \\
\hline Kentucky & Kentucky CRD 20 & 21020 & Christian & 21047 & Appalachian \\
\hline Kentucky & Kentucky CRD 50 & 21050 & Clark & 21049 & Appalachian \\
\hline Kentucky & Kentucky CRD 60 & 21060 & Clay & 21051 & Appalachian \\
\hline Kentucky & Kentucky CRD 30 & 21030 & Clinton & 21053 & Appalachian \\
\hline Kentucky & Kentucky CRD 20 & 21020 & Crittenden & 21055 & Appalachian \\
\hline Kentucky & Kentucky CRD 30 & 21030 & Cumberland & 21057 & Appalachian \\
\hline Kentucky & Kentucky CRD 20 & 21020 & Daviess & 21059 & Appalachian \\
\hline Kentucky & Kentucky CRD 30 & 21030 & Edmonson & 21061 & Appalachian \\
\hline Kentucky & Kentucky CRD 60 & 21060 & Elliott & 21063 & Appalachian \\
\hline Kentucky & Kentucky CRD 60 & 21060 & Estill & 21065 & Appalachian \\
\hline Kentucky & Kentucky CRD 50 & 21050 & Fayette & 21067 & Appalachian \\
\hline Kentucky & Kentucky CRD 50 & 21050 & Fleming & 21069 & Appalachian \\
\hline Kentucky & Kentucky CRD 60 & 21060 & Floyd & 21071 & Appalachian \\
\hline Kentucky & Kentucky CRD 50 & 21050 & Franklin & 21073 & Appalachian \\
\hline Kentucky & Kentucky CRD 10 & 21010 & Fulton & 21075 & Appalachian \\
\hline Kentucky & Kentucky CRD 40 & 21040 & Gallatin & 21077 & Appalachian \\
\hline Kentucky & Kentucky CRD 50 & 21050 & Garrard & 21079 & Appalachian \\
\hline Kentucky & Kentucky CRD 40 & 21040 & Grant & 21081 & Appalachian \\
\hline Kentucky & Kentucky CRD 10 & 21010 & Graves & 21083 & Appalachian \\
\hline Kentucky & Kentucky CRD 30 & 21030 & Grayson & 21085 & Appalachian \\
\hline Kentucky & Kentucky CRD 30 & 21030 & Green & 21087 & Appalachian \\
\hline Kentucky & Kentucky CRD 60 & 21060 & Greenup & 21089 & Appalachian \\
\hline Kentucky & Kentucky CRD 20 & 21020 & Hancock & 21091 & Appalachian \\
\hline Kentucky & Kentucky CRD 30 & 21030 & Hardin & 21093 & Appalachian \\
\hline Kentucky & Kentucky CRD 60 & 21060 & Harlan & 21095 & Appalachian \\
\hline Kentucky & Kentucky CRD 50 & 21050 & Harrison & 21097 & Appalachian \\
\hline Kentucky & Kentucky CRD 30 & 21030 & Hart & 21099 & Appalachian \\
\hline
\end{tabular}


Table 1. Crop Reporting Districts (CRD) of the conterminous United States and associated counties.-Continued

[FIPS, Federal Information Processing Standard; USDA, U.S. Department of Agriculture]

\begin{tabular}{|c|c|c|c|c|c|}
\hline State & $\begin{array}{l}\text { Crop Reporting District } \\
\text { (CRD) }\end{array}$ & CRD Identifier & County Name & County FIPS & $\begin{array}{c}\text { USDA Farm Production } \\
\text { Region }\end{array}$ \\
\hline Kentucky & Kentucky CRD 20 & 21020 & Henderson & 21101 & Appalachian \\
\hline Kentucky & Kentucky CRD 40 & 21040 & Henry & 21103 & Appalachian \\
\hline Kentucky & Kentucky CRD 10 & 21010 & Hickman & 21105 & Appalachian \\
\hline Kentucky & Kentucky CRD 20 & 21020 & Hopkins & 21107 & Appalachian \\
\hline Kentucky & Kentucky CRD 60 & 21060 & Jackson & 21109 & Appalachian \\
\hline Kentucky & Kentucky CRD 30 & 21030 & Jefferson & 21111 & Appalachian \\
\hline Kentucky & Kentucky CRD 50 & 21050 & Jessamine & 21113 & Appalachian \\
\hline Kentucky & Kentucky CRD 60 & 21060 & Johnson & 21115 & Appalachian \\
\hline Kentucky & Kentucky CRD 40 & 21040 & Kenton & 21117 & Appalachian \\
\hline Kentucky & Kentucky CRD 60 & 21060 & Knott & 21119 & Appalachian \\
\hline Kentucky & Kentucky CRD 60 & 21060 & Knox & 21121 & Appalachian \\
\hline Kentucky & Kentucky CRD 30 & 21030 & Larue & 21123 & Appalachian \\
\hline Kentucky & Kentucky CRD 60 & 21060 & Laurel & 21125 & Appalachian \\
\hline Kentucky & Kentucky CRD 60 & 21060 & Lawrence & 21127 & Appalachian \\
\hline Kentucky & Kentucky CRD 60 & 21060 & Lee & 21129 & Appalachian \\
\hline Kentucky & Kentucky CRD 60 & 21060 & Leslie & 21131 & Appalachian \\
\hline Kentucky & Kentucky CRD 60 & 21060 & Letcher & 21133 & Appalachian \\
\hline Kentucky & Kentucky CRD 60 & 21060 & Lewis & 21135 & Appalachian \\
\hline Kentucky & Kentucky CRD 50 & 21050 & Lincoln & 21137 & Appalachian \\
\hline Kentucky & Kentucky CRD 10 & 21010 & Livingston & 21139 & Appalachian \\
\hline Kentucky & Kentucky CRD 20 & 21020 & Logan & 21141 & Appalachian \\
\hline Kentucky & Kentucky CRD 10 & 21010 & Lyon & 21143 & Appalachian \\
\hline Kentucky & Kentucky CRD 10 & 21010 & McCracken & 21145 & Appalachian \\
\hline Kentucky & Kentucky CRD 60 & 21060 & McCreary & 21147 & Appalachian \\
\hline Kentucky & Kentucky CRD 20 & 21020 & McLean & 21149 & Appalachian \\
\hline Kentucky & Kentucky CRD 50 & 21050 & Madison & 21151 & Appalachian \\
\hline Kentucky & Kentucky CRD 60 & 21060 & Magoffin & 21153 & Appalachian \\
\hline Kentucky & Kentucky CRD 30 & 21030 & Marion & 21155 & Appalachian \\
\hline Kentucky & Kentucky CRD 10 & 21010 & Marshall & 21157 & Appalachian \\
\hline Kentucky & Kentucky CRD 60 & 21060 & Martin & 21159 & Appalachian \\
\hline Kentucky & Kentucky CRD 50 & 21050 & Mason & 21161 & Appalachian \\
\hline Kentucky & Kentucky CRD 30 & 21030 & Meade & 21163 & Appalachian \\
\hline Kentucky & Kentucky CRD 60 & 21060 & Menifee & 21165 & Appalachian \\
\hline Kentucky & Kentucky CRD 50 & 21050 & Mercer & 21167 & Appalachian \\
\hline Kentucky & Kentucky CRD 30 & 21030 & Metcalfe & 21169 & Appalachian \\
\hline Kentucky & Kentucky CRD 30 & 21030 & Monroe & 21171 & Appalachian \\
\hline Kentucky & Kentucky CRD 50 & 21050 & Montgomery & 21173 & Appalachian \\
\hline Kentucky & Kentucky CRD 60 & 21060 & Morgan & 21175 & Appalachian \\
\hline Kentucky & Kentucky CRD 20 & 21020 & Muhlenberg & 21177 & Appalachian \\
\hline Kentucky & Kentucky CRD 30 & 21030 & Nelson & 21179 & Appalachian \\
\hline Kentucky & Kentucky CRD 50 & 21050 & Nicholas & 21181 & Appalachian \\
\hline Kentucky & Kentucky CRD 20 & 21020 & Ohio & 21183 & Appalachian \\
\hline
\end{tabular}


Table 1. Crop Reporting Districts (CRD) of the conterminous United States and associated counties.-Continued

[FIPS, Federal Information Processing Standard; USDA, U.S. Department of Agriculture]

\begin{tabular}{|c|c|c|c|c|c|}
\hline State & $\begin{array}{l}\text { Crop Reporting District } \\
\text { (CRD) }\end{array}$ & CRD Identifier & County Name & County FIPS & $\begin{array}{c}\text { USDA Farm Production } \\
\text { Region }\end{array}$ \\
\hline Kentucky & Kentucky CRD 40 & 21040 & Oldham & 21185 & Appalachian \\
\hline Kentucky & Kentucky CRD 40 & 21040 & Owen & 21187 & Appalachian \\
\hline Kentucky & Kentucky CRD 60 & 21060 & Owsley & 21189 & Appalachian \\
\hline Kentucky & Kentucky CRD 40 & 21040 & Pendleton & 21191 & Appalachian \\
\hline Kentucky & Kentucky CRD 60 & 21060 & Perry & 21193 & Appalachian \\
\hline Kentucky & Kentucky CRD 60 & 21060 & Pike & 21195 & Appalachian \\
\hline Kentucky & Kentucky CRD 60 & 21060 & Powell & 21197 & Appalachian \\
\hline Kentucky & Kentucky CRD 60 & 21060 & Pulaski & 21199 & Appalachian \\
\hline Kentucky & Kentucky CRD 50 & 21050 & Robertson & 21201 & Appalachian \\
\hline Kentucky & Kentucky CRD 60 & 21060 & Rockcastle & 21203 & Appalachian \\
\hline Kentucky & Kentucky CRD 60 & 21060 & Rowan & 21205 & Appalachian \\
\hline Kentucky & Kentucky CRD 30 & 21030 & Russell & 21207 & Appalachian \\
\hline Kentucky & Kentucky CRD 50 & 21050 & Scott & 21209 & Appalachian \\
\hline Kentucky & Kentucky CRD 50 & 21050 & Shelby & 21211 & Appalachian \\
\hline Kentucky & Kentucky CRD 20 & 21020 & Simpson & 21213 & Appalachian \\
\hline Kentucky & Kentucky CRD 50 & 21050 & Spencer & 21215 & Appalachian \\
\hline Kentucky & Kentucky CRD 30 & 21030 & Taylor & 21217 & Appalachian \\
\hline Kentucky & Kentucky CRD 20 & 21020 & Todd & 21219 & Appalachian \\
\hline Kentucky & Kentucky CRD 10 & 21010 & Trigg & 21221 & Appalachian \\
\hline Kentucky & Kentucky CRD 40 & 21040 & Trimble & 21223 & Appalachian \\
\hline Kentucky & Kentucky CRD 20 & 21020 & Union & 21225 & Appalachian \\
\hline Kentucky & Kentucky CRD 30 & 21030 & Warren & 21227 & Appalachian \\
\hline Kentucky & Kentucky CRD 50 & 21050 & Washington & 21229 & Appalachian \\
\hline Kentucky & Kentucky CRD 60 & 21060 & Wayne & 21231 & Appalachian \\
\hline Kentucky & Kentucky CRD 20 & 21020 & Webster & 21233 & Appalachian \\
\hline Kentucky & Kentucky CRD 60 & 21060 & Whitley & 21235 & Appalachian \\
\hline Kentucky & Kentucky CRD 60 & 21060 & Wolfe & 21237 & Appalachian \\
\hline Kentucky & Kentucky CRD 50 & 21050 & Woodford & 21239 & Appalachian \\
\hline Louisiana & Louisiana CRD 70 & 22070 & Acadia & 22001 & Delta \\
\hline Louisiana & Louisiana CRD 70 & 22070 & Allen & 22003 & Delta \\
\hline Louisiana & Louisiana CRD 90 & 22090 & Ascension & 22005 & Delta \\
\hline Louisiana & Louisiana CRD 80 & 22080 & Assumption & 22007 & Delta \\
\hline Louisiana & Louisiana CRD 50 & 22050 & Avoyelles & 22009 & Delta \\
\hline Louisiana & Louisiana CRD 70 & 22070 & Beauregard & 22011 & Delta \\
\hline Louisiana & Louisiana CRD 20 & 22020 & Bienville & 22013 & Delta \\
\hline Louisiana & Louisiana CRD 10 & 22010 & Bossier & 22015 & Delta \\
\hline Louisiana & Louisiana CRD 10 & 22010 & Caddo & 22017 & Delta \\
\hline Louisiana & Louisiana CRD 70 & 22070 & Calcasieu & 22019 & Delta \\
\hline Louisiana & Louisiana CRD 20 & 22020 & Caldwell & 22021 & Delta \\
\hline Louisiana & Louisiana CRD 70 & 22070 & Cameron & 22023 & Delta \\
\hline Louisiana & Louisiana CRD 50 & 22050 & Catahoula & 22025 & Delta \\
\hline Louisiana & Louisiana CRD 20 & 22020 & Claiborne & 22027 & Delta \\
\hline
\end{tabular}


Table 1. Crop Reporting Districts (CRD) of the conterminous United States and associated counties.-Continued

[FIPS, Federal Information Processing Standard; USDA, U.S. Department of Agriculture]

\begin{tabular}{|c|c|c|c|c|c|}
\hline State & $\begin{array}{l}\text { Crop Reporting District } \\
\text { (CRD) }\end{array}$ & CRD Identifier & County Name & County FIPS & $\begin{array}{c}\text { USDA Farm Production } \\
\text { Region }\end{array}$ \\
\hline Louisiana & Louisiana CRD 50 & 22050 & Concordia & 22029 & Delta \\
\hline Louisiana & Louisiana CRD 10 & 22010 & De Soto & 22031 & Delta \\
\hline Louisiana & Louisiana CRD 60 & 22060 & East Baton Rouge & 22033 & Delta \\
\hline Louisiana & Louisiana CRD 30 & 22030 & East Carroll & 22035 & Delta \\
\hline Louisiana & Louisiana CRD 60 & 22060 & East Feliciana & 22037 & Delta \\
\hline Louisiana & Louisiana CRD 50 & 22050 & Evangeline & 22039 & Delta \\
\hline Louisiana & Louisiana CRD 30 & 22030 & Franklin & 22041 & Delta \\
\hline Louisiana & Louisiana CRD 50 & 22050 & Grant & 22043 & Delta \\
\hline Louisiana & Louisiana CRD 80 & 22080 & Iberia & 22045 & Delta \\
\hline Louisiana & Louisiana CRD 80 & 22080 & Iberville & 22047 & Delta \\
\hline Louisiana & Louisiana CRD 20 & 22020 & Jackson & 22049 & Delta \\
\hline Louisiana & Louisiana CRD 90 & 22090 & Jefferson & 22051 & Delta \\
\hline Louisiana & Louisiana CRD 70 & 22070 & Jefferson Davis & 22053 & Delta \\
\hline Louisiana & Louisiana CRD 80 & 22080 & Lafayette & 22055 & Delta \\
\hline Louisiana & Louisiana CRD 90 & 22090 & Lafourche & 22057 & Delta \\
\hline Louisiana & Louisiana CRD 50 & 22050 & La Salle & 22059 & Delta \\
\hline Louisiana & Louisiana CRD 20 & 22020 & Lincoln & 22061 & Delta \\
\hline Louisiana & Louisiana CRD 60 & 22060 & Livingston & 22063 & Delta \\
\hline Louisiana & Louisiana CRD 30 & 22030 & Madison & 22065 & Delta \\
\hline Louisiana & Louisiana CRD 30 & 22030 & Morehouse & 22067 & Delta \\
\hline Louisiana & Louisiana CRD 40 & 22040 & Natchitoches & 22069 & Delta \\
\hline Louisiana & Louisiana CRD 90 & 22090 & Orleans & 22071 & Delta \\
\hline Louisiana & Louisiana CRD 20 & 22020 & Ouachita & 22073 & Delta \\
\hline Louisiana & Louisiana CRD 90 & 22090 & Plaquemines & 22075 & Delta \\
\hline Louisiana & Louisiana CRD 50 & 22050 & Pointe Coupee & 22077 & Delta \\
\hline Louisiana & Louisiana CRD 50 & 22050 & Rapides & 22079 & Delta \\
\hline Louisiana & Louisiana CRD 10 & 22010 & Red River & 22081 & Delta \\
\hline Louisiana & Louisiana CRD 30 & 22030 & Richland & 22083 & Delta \\
\hline Louisiana & Louisiana CRD 40 & 22040 & Sabine & 22085 & Delta \\
\hline Louisiana & Louisiana CRD 90 & 22090 & St. Bernard & 22087 & Delta \\
\hline Louisiana & Louisiana CRD 90 & 22090 & St. Charles & 22089 & Delta \\
\hline Louisiana & Louisiana CRD 60 & 22060 & St. Helena & 22091 & Delta \\
\hline Louisiana & Louisiana CRD 90 & 22090 & St. James & 22093 & Delta \\
\hline Louisiana & Louisiana CRD 90 & 22090 & St. John the Baptist & 22095 & Delta \\
\hline Louisiana & Louisiana CRD 50 & 22050 & St. Landry & 22097 & Delta \\
\hline Louisiana & Louisiana CRD 80 & 22080 & St. Martin & 22099 & Delta \\
\hline Louisiana & Louisiana CRD 80 & 22080 & St. Mary & 22101 & Delta \\
\hline Louisiana & Louisiana CRD 60 & 22060 & St. Tammany & 22103 & Delta \\
\hline Louisiana & Louisiana CRD 60 & 22060 & Tangipahoa & 22105 & Delta \\
\hline Louisiana & Louisiana CRD 30 & 22030 & Tensas & 22107 & Delta \\
\hline Louisiana & Louisiana CRD 90 & 22090 & Terrebonne & 22109 & Delta \\
\hline Louisiana & Louisiana CRD 20 & 22020 & Union & 22111 & Delta \\
\hline
\end{tabular}


Table 1. Crop Reporting Districts (CRD) of the conterminous United States and associated counties. - Continued

[FIPS, Federal Information Processing Standard; USDA, U.S. Department of Agriculture]

\begin{tabular}{|c|c|c|c|c|c|}
\hline State & $\begin{array}{l}\text { Crop Reporting District } \\
\text { (CRD) }\end{array}$ & CRD Identifier & County Name & County FIPS & $\begin{array}{c}\text { USDA Farm Production } \\
\text { Region }\end{array}$ \\
\hline Louisiana & Louisiana CRD 70 & 22070 & Vermilion & 22113 & Delta \\
\hline Louisiana & Louisiana CRD 40 & 22040 & Vernon & 22115 & Delta \\
\hline Louisiana & Louisiana CRD 60 & 22060 & Washington & 22117 & Delta \\
\hline Louisiana & Louisiana CRD 10 & 22010 & Webster & 22119 & Delta \\
\hline Louisiana & Louisiana CRD 50 & 22050 & West Baton Rouge & 22121 & Delta \\
\hline Louisiana & Louisiana CRD 30 & 22030 & West Carroll & 22123 & Delta \\
\hline Louisiana & Louisiana CRD 60 & 22060 & West feliciana & 22125 & Delta \\
\hline Louisiana & Louisiana CRD 20 & 22020 & Winn & 22127 & Delta \\
\hline Maine & Maine CRD 30 & 23030 & Androscoggin & 23001 & Northeast \\
\hline Maine & Maine CRD 10 & 23010 & Aroostook & 23003 & Northeast \\
\hline Maine & Maine CRD 30 & 23030 & Cumberland & 23005 & Northeast \\
\hline Maine & Maine CRD 30 & 23030 & Franklin & 23007 & Northeast \\
\hline Maine & Maine CRD 20 & 23020 & Hancock & 23009 & Northeast \\
\hline Maine & Maine CRD 30 & 23030 & Kennebec & 23011 & Northeast \\
\hline Maine & Maine CRD 30 & 23030 & Knox & 23013 & Northeast \\
\hline Maine & Maine CRD 30 & 23030 & Lincoln & 23015 & Northeast \\
\hline Maine & Maine CRD 30 & 23030 & Oxford & 23017 & Northeast \\
\hline Maine & Maine CRD 20 & 23020 & Penobscot & 23019 & Northeast \\
\hline Maine & Maine CRD 20 & 23020 & Piscataquis & 23021 & Northeast \\
\hline Maine & Maine CRD 30 & 23030 & Sagadahoc & 23023 & Northeast \\
\hline Maine & Maine CRD 20 & 23020 & Somerset & 23025 & Northeast \\
\hline Maine & Maine CRD 20 & 23020 & Waldo & 23027 & Northeast \\
\hline Maine & Maine CRD 20 & 23020 & Washington & 23029 & Northeast \\
\hline Maine & Maine CRD 30 & 23030 & York & 23031 & Northeast \\
\hline Maryland & Maryland CRD 10 & 24010 & Allegany & 24001 & Northeast \\
\hline Maryland & Maryland CRD 80 & 24080 & Anne Arundel & 24003 & Northeast \\
\hline Maryland & Maryland CRD 20 & 24020 & Baltimore & 24005 & Northeast \\
\hline Maryland & Maryland CRD 80 & 24080 & Calvert & 24009 & Northeast \\
\hline Maryland & Maryland CRD 30 & 24030 & Caroline & 24011 & Northeast \\
\hline Maryland & Maryland CRD 20 & 24020 & Carroll & 24013 & Northeast \\
\hline Maryland & Maryland CRD 30 & 24030 & Cecil & 24015 & Northeast \\
\hline Maryland & Maryland CRD 80 & 24080 & Charles & 24017 & Northeast \\
\hline Maryland & Maryland CRD 90 & 24090 & Dorchester & 24019 & Northeast \\
\hline Maryland & Maryland CRD 20 & 24020 & Frederick & 24021 & Northeast \\
\hline Maryland & Maryland CRD 10 & 24010 & Garrett & 24023 & Northeast \\
\hline Maryland & Maryland CRD 20 & 24020 & Harford & 24025 & Northeast \\
\hline Maryland & Maryland CRD 20 & 24020 & Howard & 24027 & Northeast \\
\hline Maryland & Maryland CRD 30 & 24030 & Kent & 24029 & Northeast \\
\hline Maryland & Maryland CRD 20 & 24020 & Montgomery & 24031 & Northeast \\
\hline Maryland & Maryland CRD 80 & 24080 & Prince George's & 24033 & Northeast \\
\hline Maryland & Maryland CRD 30 & 24030 & Queen Anne's & 24035 & Northeast \\
\hline Maryland & Maryland CRD 80 & 24080 & St. Mary's & 24037 & Northeast \\
\hline
\end{tabular}


Table 1. Crop Reporting Districts (CRD) of the conterminous United States and associated counties.-Continued

[FIPS, Federal Information Processing Standard; USDA, U.S. Department of Agriculture]

\begin{tabular}{|c|c|c|c|c|c|}
\hline State & $\begin{array}{l}\text { Crop Reporting District } \\
\text { (CRD) }\end{array}$ & CRD Identifier & County Name & County FIPS & $\begin{array}{c}\text { USDA Farm Production } \\
\text { Region }\end{array}$ \\
\hline Maryland & Maryland CRD 90 & 24090 & Somerset & 24039 & Northeast \\
\hline Maryland & Maryland CRD 30 & 24030 & Talbot & 24041 & Northeast \\
\hline Maryland & Maryland CRD 20 & 24020 & Washington & 24043 & Northeast \\
\hline Maryland & Maryland CRD 90 & 24090 & Wicomico & 24045 & Northeast \\
\hline Maryland & Maryland CRD 90 & 24090 & Worcester & 24047 & Northeast \\
\hline Maryland & Maryland CRD 20 & 24020 & Baltimore & 24510 & Northeast \\
\hline Massachusetts & Massachusetts CRD 10 & 25010 & Barnstable & 25001 & Northeast \\
\hline Massachusetts & Massachusetts CRD 10 & 25010 & Berkshire & 25003 & Northeast \\
\hline Massachusetts & Massachusetts CRD 10 & 25010 & Bristol & 25005 & Northeast \\
\hline Massachusetts & Massachusetts CRD 10 & 25010 & Dukes & 25007 & Northeast \\
\hline Massachusetts & Massachusetts CRD 10 & 25010 & Essex & 25009 & Northeast \\
\hline Massachusetts & Massachusetts CRD 10 & 25010 & Franklin & 25011 & Northeast \\
\hline Massachusetts & Massachusetts CRD 10 & 25010 & Hampden & 25013 & Northeast \\
\hline Massachusetts & Massachusetts CRD 10 & 25010 & Hampshire & 25015 & Northeast \\
\hline Massachusetts & Massachusetts CRD 10 & 25010 & Middlesex & 25017 & Northeast \\
\hline Massachusetts & Massachusetts CRD 10 & 25010 & Nantucket & 25019 & Northeast \\
\hline Massachusetts & Massachusetts CRD 10 & 25010 & Norfolk & 25021 & Northeast \\
\hline Massachusetts & Massachusetts CRD 10 & 25010 & Plymouth & 25023 & Northeast \\
\hline Massachusetts & Massachusetts CRD 10 & 25010 & Suffolk & 25025 & Northeast \\
\hline Massachusetts & Massachusetts CRD 10 & 25010 & Worcester & 25027 & Northeast \\
\hline Michigan & Michigan CRD 30 & 26030 & Alcona & 26001 & Lake-states \\
\hline Michigan & Michigan CRD 10 & 26010 & Alger & 26003 & Lake-states \\
\hline Michigan & Michigan CRD 70 & 26070 & Allegan & 26005 & Lake-states \\
\hline Michigan & Michigan CRD 30 & 26030 & Alpena & 26007 & Lake-states \\
\hline Michigan & Michigan CRD 20 & 26020 & Antrim & 26009 & Lake-states \\
\hline Michigan & Michigan CRD 60 & 26060 & Arenac & 26011 & Lake-states \\
\hline Michigan & Michigan CRD 10 & 26010 & Baraga & 26013 & Lake-states \\
\hline Michigan & Michigan CRD 80 & 26080 & Barry & 26015 & Lake-states \\
\hline Michigan & Michigan CRD 60 & 26060 & Bay & 26017 & Lake-states \\
\hline Michigan & Michigan CRD 20 & 26020 & Benzie & 26019 & Lake-states \\
\hline Michigan & Michigan CRD 70 & 26070 & Berrien & 26021 & Lake-states \\
\hline Michigan & Michigan CRD 80 & 26080 & Branch & 26023 & Lake-states \\
\hline Michigan & Michigan CRD 80 & 26080 & Calhoun & 26025 & Lake-states \\
\hline Michigan & Michigan CRD 70 & 26070 & Cass & 26027 & Lake-states \\
\hline Michigan & Michigan CRD 20 & 26020 & Charlevoix & 26029 & Lake-states \\
\hline Michigan & Michigan CRD 30 & 26030 & Cheboygan & 26031 & Lake-states \\
\hline Michigan & Michigan CRD 10 & 26010 & Chippewa & 26033 & Lake-states \\
\hline Michigan & Michigan CRD 50 & 26050 & Clare & 26035 & Lake-states \\
\hline Michigan & Michigan CRD 80 & 26080 & Clinton & 26037 & Lake-states \\
\hline Michigan & Michigan CRD 30 & 26030 & Crawford & 26039 & Lake-states \\
\hline Michigan & Michigan CRD 10 & 26010 & Delta & 26041 & Lake-states \\
\hline Michigan & Michigan CRD 10 & 26010 & Dickinson & 26043 & Lake-states \\
\hline
\end{tabular}


Table 1. Crop Reporting Districts (CRD) of the conterminous United States and associated counties.-Continued

[FIPS, Federal Information Processing Standard; USDA, U.S. Department of Agriculture]

\begin{tabular}{|c|c|c|c|c|c|}
\hline State & $\begin{array}{l}\text { Crop Reporting District } \\
\text { (CRD) }\end{array}$ & CRD Identifier & County Name & County FIPS & $\begin{array}{c}\text { USDA Farm Production } \\
\text { Region }\end{array}$ \\
\hline Michigan & Michigan CRD 80 & 26080 & Eaton & 26045 & Lake-states \\
\hline Michigan & Michigan CRD 20 & 26020 & Emmet & 26047 & Lake-states \\
\hline Michigan & Michigan CRD 90 & 26090 & Genesee & 26049 & Lake-states \\
\hline Michigan & Michigan CRD 50 & 26050 & Gladwin & 26051 & Lake-states \\
\hline Michigan & Michigan CRD 10 & 26010 & Gogebic & 26053 & Lake-states \\
\hline Michigan & Michigan CRD 20 & 26020 & Grand Traverse & 26055 & Lake-states \\
\hline Michigan & Michigan CRD 50 & 26050 & Gratiot & 26057 & Lake-states \\
\hline Michigan & Michigan CRD 80 & 26080 & Hillsdale & 26059 & Lake-states \\
\hline Michigan & Michigan CRD 10 & 26010 & Houghton & 26061 & Lake-states \\
\hline Michigan & Michigan CRD 60 & 26060 & Huron & 26063 & Lake-states \\
\hline Michigan & Michigan CRD 80 & 26080 & Ingham & 26065 & Lake-states \\
\hline Michigan & Michigan CRD 80 & 26080 & Ionia & 26067 & Lake-states \\
\hline Michigan & Michigan CRD 30 & 26030 & Iosco & 26069 & Lake-states \\
\hline Michigan & Michigan CRD 10 & 26010 & Iron & 26071 & Lake-states \\
\hline Michigan & Michigan CRD 50 & 26050 & Isabella & 26073 & Lake-states \\
\hline Michigan & Michigan CRD 80 & 26080 & Jackson & 26075 & Lake-states \\
\hline Michigan & Michigan CRD 70 & 26070 & Kalamazoo & 26077 & Lake-states \\
\hline Michigan & Michigan CRD 20 & 26020 & Kalkaska & 26079 & Lake-states \\
\hline Michigan & Michigan CRD 70 & 26070 & Kent & 26081 & Lake-states \\
\hline Michigan & Michigan CRD 10 & 26010 & Keweenaw & 26083 & Lake-states \\
\hline Michigan & Michigan CRD 40 & 26040 & Lake & 26085 & Lake-states \\
\hline Michigan & Michigan CRD 90 & 26090 & Lapeer & 26087 & Lake-states \\
\hline Michigan & Michigan CRD 20 & 26020 & Leelanau & 26089 & Lake-states \\
\hline Michigan & Michigan CRD 90 & 26090 & Lenawee & 26091 & Lake-states \\
\hline Michigan & Michigan CRD 90 & 26090 & Livingston & 26093 & Lake-states \\
\hline Michigan & Michigan CRD 10 & 26010 & Luce & 26095 & Lake-states \\
\hline Michigan & Michigan CRD 10 & 26010 & Mackinac & 26097 & Lake-states \\
\hline Michigan & Michigan CRD 90 & 26090 & Macomb & 26099 & Lake-states \\
\hline Michigan & Michigan CRD 20 & 26020 & Manistee & 26101 & Lake-states \\
\hline Michigan & Michigan CRD 10 & 26010 & Marquette & 26103 & Lake-states \\
\hline Michigan & Michigan CRD 40 & 26040 & Mason & 26105 & Lake-states \\
\hline Michigan & Michigan CRD 50 & 26050 & Mecosta & 26107 & Lake-states \\
\hline Michigan & Michigan CRD 10 & 26010 & Menominee & 26109 & Lake-states \\
\hline Michigan & Michigan CRD 50 & 26050 & Midland & 26111 & Lake-states \\
\hline Michigan & Michigan CRD 20 & 26020 & Missaukee & 26113 & Lake-states \\
\hline Michigan & Michigan CRD 90 & 26090 & Monroe & 26115 & Lake-states \\
\hline Michigan & Michigan CRD 50 & 26050 & Montcalm & 26117 & Lake-states \\
\hline Michigan & Michigan CRD 30 & 26030 & Montmorency & 26119 & Lake-states \\
\hline Michigan & Michigan CRD 40 & 26040 & Presque Isle & 26121 & Lake-states \\
\hline Michigan & Michigan CRD 40 & 26040 & Newaygo & 26123 & Lake-states \\
\hline Michigan & Michigan CRD 90 & 26090 & Oakland & 26125 & Lake-states \\
\hline Michigan & Michigan CRD 40 & 26040 & Oceana & 26127 & Lake-states \\
\hline
\end{tabular}


Table 1. Crop Reporting Districts (CRD) of the conterminous United States and associated counties.-Continued

[FIPS, Federal Information Processing Standard; USDA, U.S. Department of Agriculture]

\begin{tabular}{|c|c|c|c|c|c|}
\hline State & $\begin{array}{l}\text { Crop Reporting District } \\
\text { (CRD) }\end{array}$ & CRD Identifier & County Name & County FIPS & $\begin{array}{c}\text { USDA Farm Production } \\
\text { Region }\end{array}$ \\
\hline Michigan & Michigan CRD 30 & 26030 & Ogemaw & 26129 & Lake-states \\
\hline Michigan & Michigan CRD 10 & 26010 & Ontonagon & 26131 & Lake-states \\
\hline Michigan & Michigan CRD 50 & 26050 & Osceola & 26133 & Lake-states \\
\hline Michigan & Michigan CRD 30 & 26030 & Oscoda & 26135 & Lake-states \\
\hline Michigan & Michigan CRD 30 & 26030 & Otsego & 26137 & Lake-states \\
\hline Michigan & Michigan CRD 70 & 26070 & Ottawa & 26139 & Lake-states \\
\hline Michigan & Michigan CRD 30 & 26030 & Presque Isle & 26141 & Lake-states \\
\hline Michigan & Michigan CRD 30 & 26030 & Roscommon & 26143 & Lake-states \\
\hline Michigan & Michigan CRD 60 & 26060 & Saginaw & 26145 & Lake-states \\
\hline Michigan & Michigan CRD 90 & 26090 & St. Clair & 26147 & Lake-states \\
\hline Michigan & Michigan CRD 80 & 26080 & St. Joseph & 26149 & Lake-states \\
\hline Michigan & Michigan CRD 60 & 26060 & Sanilac & 26151 & Lake-states \\
\hline Michigan & Michigan CRD 10 & 26010 & Schoolcraft & 26153 & Lake-states \\
\hline Michigan & Michigan CRD 80 & 26080 & Shiawassee & 26155 & Lake-states \\
\hline Michigan & Michigan CRD 60 & 26060 & Tuscola & 26157 & Lake-states \\
\hline Michigan & Michigan CRD 70 & 26070 & Van Buren & 26159 & Lake-states \\
\hline Michigan & Michigan CRD 90 & 26090 & Washtenaw & 26161 & Lake-states \\
\hline Michigan & Michigan CRD 90 & 26090 & Wayne & 26163 & Lake-states \\
\hline Michigan & Michigan CRD 20 & 26020 & Wexford & 26165 & Lake-states \\
\hline Minnesota & Minnesota CRD 60 & 27060 & Aitkin & 27001 & Lake-states \\
\hline Minnesota & Minnesota CRD 60 & 27060 & Anoka & 27003 & Lake-states \\
\hline Minnesota & Minnesota CRD 10 & 27010 & Becker & 27005 & Lake-states \\
\hline Minnesota & Minnesota CRD 20 & 27020 & Beltrami & 27007 & Lake-states \\
\hline Minnesota & Minnesota CRD 50 & 27050 & Benton & 27009 & Lake-states \\
\hline Minnesota & Minnesota CRD 40 & 27040 & Big Stone & 27011 & Lake-states \\
\hline Minnesota & Minnesota CRD 80 & 27080 & Blue Earth & 27013 & Lake-states \\
\hline Minnesota & Minnesota CRD 80 & 27080 & Brown & 27015 & Lake-states \\
\hline Minnesota & Minnesota CRD 60 & 27060 & Carlton & 27017 & Lake-states \\
\hline Minnesota & Minnesota CRD 50 & 27050 & Carver & 27019 & Lake-states \\
\hline Minnesota & Minnesota CRD 20 & 27020 & Cass & 27021 & Lake-states \\
\hline Minnesota & Minnesota CRD 40 & 27040 & Chippewa & 27023 & Lake-states \\
\hline Minnesota & Minnesota CRD 60 & 27060 & Chisago & 27025 & Lake-states \\
\hline Minnesota & Minnesota CRD 10 & 27010 & Clay & 27027 & Lake-states \\
\hline Minnesota & Minnesota CRD 10 & 27010 & Clearwater & 27029 & Lake-states \\
\hline Minnesota & Minnesota CRD 30 & 27030 & Cook & 27031 & Lake-states \\
\hline Minnesota & Minnesota CRD 70 & 27070 & Cottonwood & 27033 & Lake-states \\
\hline Minnesota & Minnesota CRD 60 & 27060 & Crow Wing & 27035 & Lake-states \\
\hline Minnesota & Minnesota CRD 90 & 27090 & Dakota & 27037 & Lake-states \\
\hline Minnesota & Minnesota CRD 90 & 27090 & Dodge & 27039 & Lake-states \\
\hline Minnesota & Minnesota CRD 40 & 27040 & Douglas & 27041 & Lake-states \\
\hline Minnesota & Minnesota CRD 80 & 27080 & Faribault & 27043 & Lake-states \\
\hline Minnesota & Minnesota CRD 90 & 27090 & Fillmore & 27045 & Lake-states \\
\hline
\end{tabular}


Table 1. Crop Reporting Districts (CRD) of the conterminous United States and associated counties. - Continued

[FIPS, Federal Information Processing Standard; USDA, U.S. Department of Agriculture]

\begin{tabular}{|c|c|c|c|c|c|}
\hline State & $\begin{array}{l}\text { Crop Reporting District } \\
\text { (CRD) }\end{array}$ & CRD Identifier & County Name & County FIPS & $\begin{array}{c}\text { USDA Farm Production } \\
\text { Region }\end{array}$ \\
\hline Minnesota & Minnesota CRD 80 & 27080 & Freeborn & 27047 & Lake-states \\
\hline Minnesota & Minnesota CRD 90 & 27090 & Goodhue & 27049 & Lake-states \\
\hline Minnesota & Minnesota CRD 40 & 27040 & Grant & 27051 & Lake-states \\
\hline Minnesota & Minnesota CRD 60 & 27060 & Hennepin & 27053 & Lake-states \\
\hline Minnesota & Minnesota CRD 90 & 27090 & Houston & 27055 & Lake-states \\
\hline Minnesota & Minnesota CRD 20 & 27020 & Hubbard & 27057 & Lake-states \\
\hline Minnesota & Minnesota CRD 60 & 27060 & Isanti & 27059 & Lake-states \\
\hline Minnesota & Minnesota CRD 20 & 27020 & Itasca & 27061 & Lake-states \\
\hline Minnesota & Minnesota CRD 70 & 27070 & Jackson & 27063 & Lake-states \\
\hline Minnesota & Minnesota CRD 60 & 27060 & Kanabec & 27065 & Lake-states \\
\hline Minnesota & Minnesota CRD 50 & 27050 & Kandiyohi & 27067 & Lake-states \\
\hline Minnesota & Minnesota CRD 10 & 27010 & Kittson & 27069 & Lake-states \\
\hline Minnesota & Minnesota CRD 20 & 27020 & Koochiching & 27071 & Lake-states \\
\hline Minnesota & Minnesota CRD 40 & 27040 & Lac Qui Parle & 27073 & Lake-states \\
\hline Minnesota & Minnesota CRD 30 & 27030 & Lake & 27075 & Lake-states \\
\hline Minnesota & Minnesota CRD 20 & 27020 & Lake of the Woods & 27077 & Lake-states \\
\hline Minnesota & Minnesota CRD 80 & 27080 & Le Sueur & 27079 & Lake-states \\
\hline Minnesota & Minnesota CRD 70 & 27070 & Lincoln & 27081 & Lake-states \\
\hline Minnesota & Minnesota CRD 70 & 27070 & Lyon & 27083 & Lake-states \\
\hline Minnesota & Minnesota CRD 50 & 27050 & McLeod & 27085 & Lake-states \\
\hline Minnesota & Minnesota CRD 10 & 27010 & Mahnomen & 27087 & Lake-states \\
\hline Minnesota & Minnesota CRD 10 & 27010 & Marshall & 27089 & Lake-states \\
\hline Minnesota & Minnesota CRD 80 & 27080 & Martin & 27091 & Lake-states \\
\hline Minnesota & Minnesota CRD 50 & 27050 & Meeker & 27093 & Lake-states \\
\hline Minnesota & Minnesota CRD 60 & 27060 & Mille Lacs & 27095 & Lake-states \\
\hline Minnesota & Minnesota CRD 50 & 27050 & Morrison & 27097 & Lake-states \\
\hline Minnesota & Minnesota CRD 90 & 27090 & Mower & 27099 & Lake-states \\
\hline Minnesota & Minnesota CRD 70 & 27070 & Murray & 27101 & Lake-states \\
\hline Minnesota & Minnesota CRD 80 & 27080 & Nicollet & 27103 & Lake-states \\
\hline Minnesota & Minnesota CRD 70 & 27070 & Nobles & 27105 & Lake-states \\
\hline Minnesota & Minnesota CRD 10 & 27010 & Norman & 27107 & Lake-states \\
\hline Minnesota & Minnesota CRD 90 & 27090 & Olmsted & 27109 & Lake-states \\
\hline Minnesota & Minnesota CRD 40 & 27040 & Otter Tail & 27111 & Lake-states \\
\hline Minnesota & Minnesota CRD 10 & 27010 & Pennington & 27113 & Lake-states \\
\hline Minnesota & Minnesota CRD 60 & 27060 & Pine & 27115 & Lake-states \\
\hline Minnesota & Minnesota CRD 70 & 27070 & Pipestone & 27117 & Lake-states \\
\hline Minnesota & Minnesota CRD 10 & 27010 & Polk & 27119 & Lake-states \\
\hline Minnesota & Minnesota CRD 40 & 27040 & Pope & 27121 & Lake-states \\
\hline Minnesota & Minnesota CRD 60 & 27060 & Ramsey & 27123 & Lake-states \\
\hline Minnesota & Minnesota CRD 10 & 27010 & Red Lake & 27125 & Lake-states \\
\hline Minnesota & Minnesota CRD 70 & 27070 & Redwood & 27127 & Lake-states \\
\hline Minnesota & Minnesota CRD 50 & 27050 & Renville & 27129 & Lake-states \\
\hline
\end{tabular}


Table 1. Crop Reporting Districts (CRD) of the conterminous United States and associated counties.-Continued

[FIPS, Federal Information Processing Standard; USDA, U.S. Department of Agriculture]

\begin{tabular}{|c|c|c|c|c|c|}
\hline State & $\begin{array}{l}\text { Crop Reporting District } \\
\text { (CRD) }\end{array}$ & CRD Identifier & County Name & County FIPS & $\begin{array}{c}\text { USDA Farm Production } \\
\text { Region }\end{array}$ \\
\hline Minnesota & Minnesota CRD 80 & 27080 & Rice & 27131 & Lake-states \\
\hline Minnesota & Minnesota CRD 70 & 27070 & Rock & 27133 & Lake-states \\
\hline Minnesota & Minnesota CRD 10 & 27010 & Roseau & 27135 & Lake-states \\
\hline Minnesota & Minnesota CRD 30 & 27030 & St. Louis & 27137 & Lake-states \\
\hline Minnesota & Minnesota CRD 50 & 27050 & Scott & 27139 & Lake-states \\
\hline Minnesota & Minnesota CRD 50 & 27050 & Sherburne & 27141 & Lake-states \\
\hline Minnesota & Minnesota CRD 50 & 27050 & Sibley & 27143 & Lake-states \\
\hline Minnesota & Minnesota CRD 50 & 27050 & Stearns & 27145 & Lake-states \\
\hline Minnesota & Minnesota CRD 80 & 27080 & Steele & 27147 & Lake-states \\
\hline Minnesota & Minnesota CRD 40 & 27040 & Stevens & 27149 & Lake-states \\
\hline Minnesota & Minnesota CRD 40 & 27040 & Swift & 27151 & Lake-states \\
\hline Minnesota & Minnesota CRD 50 & 27050 & Todd & 27153 & Lake-states \\
\hline Minnesota & Minnesota CRD 40 & 27040 & Traverse & 27155 & Lake-states \\
\hline Minnesota & Minnesota CRD 90 & 27090 & Wabasha & 27157 & Lake-states \\
\hline Minnesota & Minnesota CRD 50 & 27050 & Wadena & 27159 & Lake-states \\
\hline Minnesota & Minnesota CRD 80 & 27080 & Waseca & 27161 & Lake-states \\
\hline Minnesota & Minnesota CRD 60 & 27060 & Washington & 27163 & Lake-states \\
\hline Minnesota & Minnesota CRD 80 & 27080 & Watonwan & 27165 & Lake-states \\
\hline Minnesota & Minnesota CRD 40 & 27040 & Wilkin & 27167 & Lake-states \\
\hline Minnesota & Minnesota CRD 90 & 27090 & Winona & 27169 & Lake-states \\
\hline Minnesota & Minnesota CRD 50 & 27050 & Wright & 27171 & Lake-states \\
\hline Minnesota & Minnesota CRD 40 & 27040 & Yellow Medicine & 27173 & Lake-states \\
\hline Mississippi & Mississippi CRD 70 & 28070 & Adams & 28001 & Delta \\
\hline Mississippi & Mississippi CRD 30 & 28030 & Alcorn & 28003 & Delta \\
\hline Mississippi & Mississippi CRD 70 & 28070 & Amite & 28005 & Delta \\
\hline Mississippi & Mississippi CRD 50 & 28050 & Attala & 28007 & Delta \\
\hline Mississippi & Mississippi CRD 20 & 28020 & Benton & 28009 & Delta \\
\hline Mississippi & Mississippi CRD 10 & 28010 & Bolivar & 28011 & Delta \\
\hline Mississippi & Mississippi CRD 20 & 28020 & Calhoun & 28013 & Delta \\
\hline Mississippi & Mississippi CRD 50 & 28050 & Carroll & 28015 & Delta \\
\hline Mississippi & Mississippi CRD 60 & 28060 & Chickasaw & 28017 & Delta \\
\hline Mississippi & Mississippi CRD 50 & 28050 & Choctaw & 28019 & Delta \\
\hline Mississippi & Mississippi CRD 70 & 28070 & Claiborne & 28021 & Delta \\
\hline Mississippi & Mississippi CRD 90 & 28090 & Clarke & 28023 & Delta \\
\hline Mississippi & Mississippi CRD 60 & 28060 & Clay & 28025 & Delta \\
\hline Mississippi & Mississippi CRD 10 & 28010 & Coahoma & 28027 & Delta \\
\hline Mississippi & Mississippi CRD 70 & 28070 & Copiah & 28029 & Delta \\
\hline Mississippi & Mississippi CRD 80 & 28080 & Covington & 28031 & Delta \\
\hline Mississippi & Mississippi CRD 20 & 28020 & De Soto & 28033 & Delta \\
\hline Mississippi & Mississippi CRD 90 & 28090 & Forrest & 28035 & Delta \\
\hline Mississippi & Mississippi CRD 70 & 28070 & Franklin & 28037 & Delta \\
\hline Mississippi & Mississippi CRD 90 & 28090 & George & 28039 & Delta \\
\hline
\end{tabular}


Table 1. Crop Reporting Districts (CRD) of the conterminous United States and associated counties.-Continued

[FIPS, Federal Information Processing Standard; USDA, U.S. Department of Agriculture]

\begin{tabular}{|c|c|c|c|c|c|}
\hline State & $\begin{array}{l}\text { Crop Reporting District } \\
\text { (CRD) }\end{array}$ & CRD Identifier & County Name & County FIPS & $\begin{array}{c}\text { USDA Farm Production } \\
\text { Region }\end{array}$ \\
\hline Mississippi & Mississippi CRD 90 & 28090 & Greene & 28041 & Delta \\
\hline Mississippi & Mississippi CRD 20 & 28020 & Grenada & 28043 & Delta \\
\hline Mississippi & Mississippi CRD 90 & 28090 & Hancock & 28045 & Delta \\
\hline Mississippi & Mississippi CRD 90 & 28090 & Harrison & 28047 & Delta \\
\hline Mississippi & Mississippi CRD 70 & 28070 & Hinds & 28049 & Delta \\
\hline Mississippi & Mississippi CRD 50 & 28050 & Holmes & 28051 & Delta \\
\hline Mississippi & Mississippi CRD 40 & 28040 & Humphreys & 28053 & Delta \\
\hline Mississippi & Mississippi CRD 40 & 28040 & Issaquena & 28055 & Delta \\
\hline Mississippi & Mississippi CRD 30 & 28030 & Itawamba & 28057 & Delta \\
\hline Mississippi & Mississippi CRD 90 & 28090 & Jackson & 28059 & Delta \\
\hline Mississippi & Mississippi CRD 90 & 28090 & Jasper & 28061 & Delta \\
\hline Mississippi & Mississippi CRD 70 & 28070 & Jefferson & 28063 & Delta \\
\hline Mississippi & Mississippi CRD 80 & 28080 & Jefferson Davis & 28065 & Delta \\
\hline Mississippi & Mississippi CRD 90 & 28090 & Jones & 28067 & Delta \\
\hline Mississippi & Mississippi CRD 60 & 28060 & Kemper & 28069 & Delta \\
\hline Mississippi & Mississippi CRD 20 & 28020 & Lafayette & 28071 & Delta \\
\hline Mississippi & Mississippi CRD 80 & 28080 & Lamar & 28073 & Delta \\
\hline Mississippi & Mississippi CRD 90 & 28090 & Lauderdale & 28075 & Delta \\
\hline Mississippi & Mississippi CRD 80 & 28080 & Lawrence & 28077 & Delta \\
\hline Mississippi & Mississippi CRD 50 & 28050 & Leake & 28079 & Delta \\
\hline Mississippi & Mississippi CRD 30 & 28030 & Lee & 28081 & Delta \\
\hline Mississippi & Mississippi CRD 40 & 28040 & Leflore & 28083 & Delta \\
\hline Mississippi & Mississippi CRD 70 & 28070 & Lincoln & 28085 & Delta \\
\hline Mississippi & Mississippi CRD 60 & 28060 & Lowndes & 28087 & Delta \\
\hline Mississippi & Mississippi CRD 50 & 28050 & Madison & 28089 & Delta \\
\hline Mississippi & Mississippi CRD 80 & 28080 & Marion & 28091 & Delta \\
\hline Mississippi & Mississippi CRD 20 & 28020 & Marshall & 28093 & Delta \\
\hline Mississippi & Mississippi CRD 60 & 28060 & Monroe & 28095 & Delta \\
\hline Mississippi & Mississippi CRD 50 & 28050 & Montgomery & 28097 & Delta \\
\hline Mississippi & Mississippi CRD 60 & 28060 & Neshoba & 28099 & Delta \\
\hline Mississippi & Mississippi CRD 90 & 28090 & Newton & 28101 & Delta \\
\hline Mississippi & Mississippi CRD 60 & 28060 & Noxubee & 28103 & Delta \\
\hline Mississippi & Mississippi CRD 60 & 28060 & Oktibbeha & 28105 & Delta \\
\hline Mississippi & Mississippi CRD 20 & 28020 & Panola & 28107 & Delta \\
\hline Mississippi & Mississippi CRD 90 & 28090 & Pearl River & 28109 & Delta \\
\hline Mississippi & Mississippi CRD 90 & 28090 & Perry & 28111 & Delta \\
\hline Mississippi & Mississippi CRD 80 & 28080 & Pike & 28113 & Delta \\
\hline Mississippi & Mississippi CRD 30 & 28030 & Pontotoc & 28115 & Delta \\
\hline Mississippi & Mississippi CRD 30 & 28030 & Prentiss & 28117 & Delta \\
\hline Mississippi & Mississippi CRD 10 & 28010 & Quitman & 28119 & Delta \\
\hline Mississippi & Mississippi CRD 50 & 28050 & Rankin & 28121 & Delta \\
\hline Mississippi & Mississippi CRD 50 & 28050 & Scott & 28123 & Delta \\
\hline
\end{tabular}


Table 1. Crop Reporting Districts (CRD) of the conterminous United States and associated counties.—Continued

[FIPS, Federal Information Processing Standard; USDA, U.S. Department of Agriculture]

\begin{tabular}{|c|c|c|c|c|c|}
\hline State & $\begin{array}{l}\text { Crop Reporting District } \\
\text { (CRD) }\end{array}$ & CRD Identifier & County Name & County FIPS & $\begin{array}{l}\text { USDA Farm Production } \\
\text { Region }\end{array}$ \\
\hline Mississippi & Mississippi CRD 40 & 28040 & Sharkey & 28125 & Delta \\
\hline Mississippi & Mississippi CRD 80 & 28080 & Simpson & 28127 & Delta \\
\hline Mississippi & Mississippi CRD 80 & 28080 & Smith & 28129 & Delta \\
\hline Mississippi & Mississippi CRD 90 & 28090 & Stone & 28131 & Delta \\
\hline Mississippi & Mississippi CRD 40 & 28040 & Sunflower & 28133 & Delta \\
\hline Mississippi & Mississippi CRD 10 & 28010 & Tallahatchie & 28135 & Delta \\
\hline Mississippi & Mississippi CRD 20 & 28020 & Tate & 28137 & Delta \\
\hline Mississippi & Mississippi CRD 30 & 28030 & Tippah & 28139 & Delta \\
\hline Mississippi & Mississippi CRD 30 & 28030 & Tishomingo & 28141 & Delta \\
\hline Mississippi & Mississippi CRD 10 & 28010 & Tunica & 28143 & Delta \\
\hline Mississippi & Mississippi CRD 30 & 28030 & Union & 28145 & Delta \\
\hline Mississippi & Mississippi CRD 80 & 28080 & Walthall & 28147 & Delta \\
\hline Mississippi & Mississippi CRD 70 & 28070 & Warren & 28149 & Delta \\
\hline Mississippi & Mississippi CRD 40 & 28040 & Washington & 28151 & Delta \\
\hline Mississippi & Mississippi CRD 90 & 28090 & Wayne & 28153 & Delta \\
\hline Mississippi & Mississippi CRD 50 & 28050 & Webster & 28155 & Delta \\
\hline Mississippi & Mississippi CRD 70 & 28070 & Wilkinson & 28157 & Delta \\
\hline Mississippi & Mississippi CRD 60 & 28060 & Winston & 28159 & Delta \\
\hline Mississippi & Mississippi CRD 20 & 28020 & Yalobusha & 28161 & Delta \\
\hline Mississippi & Mississippi CRD 40 & 28040 & Yazoo & 28163 & Delta \\
\hline Missouri & Missouri CRD 20 & 29020 & Adair & 29001 & Corn-belt \\
\hline Missouri & Missouri CRD 10 & 29010 & Andrew & 29003 & Corn-belt \\
\hline Missouri & Missouri CRD 10 & 29010 & Atchison & 29005 & Corn-belt \\
\hline Missouri & Missouri CRD 30 & 29030 & Audrain & 29007 & Corn-belt \\
\hline Missouri & Missouri CRD 70 & 29070 & Barry & 29009 & Corn-belt \\
\hline Missouri & Missouri CRD 70 & 29070 & Barton & 29011 & Corn-belt \\
\hline Missouri & Missouri CRD 40 & 29040 & Bates & 29013 & Corn-belt \\
\hline Missouri & Missouri CRD 50 & 29050 & Benton & 29015 & Corn-belt \\
\hline Missouri & Missouri CRD 80 & 29080 & Bollinger & 29017 & Corn-belt \\
\hline Missouri & Missouri CRD 50 & 29050 & Boone & 29019 & Corn-belt \\
\hline Missouri & Missouri CRD 10 & 29010 & Buchanan & 29021 & Corn-belt \\
\hline Missouri & Missouri CRD 90 & 29090 & Butler & 29023 & Corn-belt \\
\hline Missouri & Missouri CRD 10 & 29010 & Caldwell & 29025 & Corn-belt \\
\hline Missouri & Missouri CRD 50 & 29050 & Callaway & 29027 & Corn-belt \\
\hline Missouri & Missouri CRD 50 & 29050 & Camden & 29029 & Corn-belt \\
\hline Missouri & Missouri CRD 90 & 29090 & Cape Girardeau & 29031 & Corn-belt \\
\hline Missouri & Missouri CRD 20 & 29020 & Carroll & 29033 & Corn-belt \\
\hline Missouri & Missouri CRD 80 & 29080 & Carter & 29035 & Corn-belt \\
\hline Missouri & Missouri CRD 40 & 29040 & Cass & 29037 & Corn-belt \\
\hline Missouri & Missouri CRD 40 & 29040 & Cedar & 29039 & Corn-belt \\
\hline Missouri & Missouri CRD 20 & 29020 & Chariton & 29041 & Corn-belt \\
\hline Missouri & Missouri CRD 70 & 29070 & Christian & 29043 & Corn-belt \\
\hline
\end{tabular}


Table 1. Crop Reporting Districts (CRD) of the conterminous United States and associated counties.-Continued

[FIPS, Federal Information Processing Standard; USDA, U.S. Department of Agriculture]

\begin{tabular}{|c|c|c|c|c|c|}
\hline State & $\begin{array}{l}\text { Crop Reporting District } \\
\text { (CRD) }\end{array}$ & CRD Identifier & County Name & County FIPS & $\begin{array}{c}\text { USDA Farm Production } \\
\text { Region }\end{array}$ \\
\hline Missouri & Missouri CRD 30 & 29030 & Clark & 29045 & Corn-belt \\
\hline Missouri & Missouri CRD 10 & 29010 & Clay & 29047 & Corn-belt \\
\hline Missouri & Missouri CRD 10 & 29010 & Clinton & 29049 & Corn-belt \\
\hline Missouri & Missouri CRD 50 & 29050 & Cole & 29051 & Corn-belt \\
\hline Missouri & Missouri CRD 50 & 29050 & Cooper & 29053 & Corn-belt \\
\hline Missouri & Missouri CRD 60 & 29060 & Crawford & 29055 & Corn-belt \\
\hline Missouri & Missouri CRD 70 & 29070 & Dade & 29057 & Corn-belt \\
\hline Missouri & Missouri CRD 50 & 29050 & Dallas & 29059 & Corn-belt \\
\hline Missouri & Missouri CRD 10 & 29010 & Daviess & 29061 & Corn-belt \\
\hline Missouri & Missouri CRD 10 & 29010 & De kalb & 29063 & Corn-belt \\
\hline Missouri & Missouri CRD 80 & 29080 & Dent & 29065 & Corn-belt \\
\hline Missouri & Missouri CRD 80 & 29080 & Douglas & 29067 & Corn-belt \\
\hline Missouri & Missouri CRD 90 & 29090 & Dunklin & 29069 & Corn-belt \\
\hline Missouri & Missouri CRD 60 & 29060 & Franklin & 29071 & Corn-belt \\
\hline Missouri & Missouri CRD 60 & 29060 & Gasconade & 29073 & Corn-belt \\
\hline Missouri & Missouri CRD 10 & 29010 & Gentry & 29075 & Corn-belt \\
\hline Missouri & Missouri CRD 70 & 29070 & Greene & 29077 & Corn-belt \\
\hline Missouri & Missouri CRD 20 & 29020 & Grundy & 29079 & Corn-belt \\
\hline Missouri & Missouri CRD 10 & 29010 & Harrison & 29081 & Corn-belt \\
\hline Missouri & Missouri CRD 40 & 29040 & Henry & 29083 & Corn-belt \\
\hline Missouri & Missouri CRD 50 & 29050 & Hickory & 29085 & Corn-belt \\
\hline Missouri & Missouri CRD 10 & 29010 & Holt & 29087 & Corn-belt \\
\hline Missouri & Missouri CRD 50 & 29050 & Howard & 29089 & Corn-belt \\
\hline Missouri & Missouri CRD 80 & 29080 & Howell & 29091 & Corn-belt \\
\hline Missouri & Missouri CRD 80 & 29080 & Iron & 29093 & Corn-belt \\
\hline Missouri & Missouri CRD 40 & 29040 & Jackson & 29095 & Corn-belt \\
\hline Missouri & Missouri CRD 70 & 29070 & Jasper & 29097 & Corn-belt \\
\hline Missouri & Missouri CRD 60 & 29060 & Jefferson & 29099 & Corn-belt \\
\hline Missouri & Missouri CRD 40 & 29040 & Johnson & 29101 & Corn-belt \\
\hline Missouri & Missouri CRD 30 & 29030 & Knox & 29103 & Corn-belt \\
\hline Missouri & Missouri CRD 50 & 29050 & Laclede & 29105 & Corn-belt \\
\hline Missouri & Missouri CRD 40 & 29040 & Lafayette & 29107 & Corn-belt \\
\hline Missouri & Missouri CRD 70 & 29070 & Lawrence & 29109 & Corn-belt \\
\hline Missouri & Missouri CRD 30 & 29030 & Lewis & 29111 & Corn-belt \\
\hline Missouri & Missouri CRD 60 & 29060 & Lincoln & 29113 & Corn-belt \\
\hline Missouri & Missouri CRD 20 & 29020 & Linn & 29115 & Corn-belt \\
\hline Missouri & Missouri CRD 20 & 29020 & Livingston & 29117 & Corn-belt \\
\hline Missouri & Missouri CRD 70 & 29070 & McDonald & 29119 & Corn-belt \\
\hline Missouri & Missouri CRD 20 & 29020 & Macon & 29121 & Corn-belt \\
\hline Missouri & Missouri CRD 80 & 29080 & Madison & 29123 & Corn-belt \\
\hline Missouri & Missouri CRD 50 & 29050 & Maries & 29125 & Corn-belt \\
\hline Missouri & Missouri CRD 30 & 29030 & Marion & 29127 & Corn-belt \\
\hline
\end{tabular}


Table 1. Crop Reporting Districts (CRD) of the conterminous United States and associated counties.—Continued

[FIPS, Federal Information Processing Standard; USDA, U.S. Department of Agriculture]

\begin{tabular}{|c|c|c|c|c|c|}
\hline State & $\begin{array}{l}\text { Crop Reporting District } \\
\text { (CRD) }\end{array}$ & CRD Identifier & County Name & County FIPS & $\begin{array}{c}\text { USDA Farm Production } \\
\text { Region }\end{array}$ \\
\hline Missouri & Missouri CRD 20 & 29020 & Mercer & 29129 & Corn-belt \\
\hline Missouri & Missouri CRD 50 & 29050 & Miller & 29131 & Corn-belt \\
\hline Missouri & Missouri CRD 90 & 29090 & Mississippi & 29133 & Corn-belt \\
\hline Missouri & Missouri CRD 50 & 29050 & Moniteau & 29135 & Corn-belt \\
\hline Missouri & Missouri CRD 30 & 29030 & Monroe & 29137 & Corn-belt \\
\hline Missouri & Missouri CRD 60 & 29060 & Montgomery & 29139 & Corn-belt \\
\hline Missouri & Missouri CRD 50 & 29050 & Morgan & 29141 & Corn-belt \\
\hline Missouri & Missouri CRD 90 & 29090 & New Madrid & 29143 & Corn-belt \\
\hline Missouri & Missouri CRD 70 & 29070 & Newton & 29145 & Corn-belt \\
\hline Missouri & Missouri CRD 10 & 29010 & Nodaway & 29147 & Corn-belt \\
\hline Missouri & Missouri CRD 80 & 29080 & Oregon & 29149 & Corn-belt \\
\hline Missouri & Missouri CRD 50 & 29050 & Osage & 29151 & Corn-belt \\
\hline Missouri & Missouri CRD 80 & 29080 & Ozark & 29153 & Corn-belt \\
\hline Missouri & Missouri CRD 90 & 29090 & Pemiscot & 29155 & Corn-belt \\
\hline Missouri & Missouri CRD 60 & 29060 & Perry & 29157 & Corn-belt \\
\hline Missouri & Missouri CRD 50 & 29050 & Pettis & 29159 & Corn-belt \\
\hline Missouri & Missouri CRD 50 & 29050 & Phelps & 29161 & Corn-belt \\
\hline Missouri & Missouri CRD 30 & 29030 & Pike & 29163 & Corn-belt \\
\hline Missouri & Missouri CRD 10 & 29010 & Platte & 29165 & Corn-belt \\
\hline Missouri & Missouri CRD 50 & 29050 & Polk & 29167 & Corn-belt \\
\hline Missouri & Missouri CRD 50 & 29050 & Pulaski & 29169 & Corn-belt \\
\hline Missouri & Missouri CRD 20 & 29020 & Putnam & 29171 & Corn-belt \\
\hline Missouri & Missouri CRD 30 & 29030 & Ralls & 29173 & Corn-belt \\
\hline Missouri & Missouri CRD 20 & 29020 & Randolph & 29175 & Corn-belt \\
\hline Missouri & Missouri CRD 10 & 29010 & Ray & 29177 & Corn-belt \\
\hline Missouri & Missouri CRD 80 & 29080 & Reynolds & 29179 & Corn-belt \\
\hline Missouri & Missouri CRD 80 & 29080 & Ripley & 29181 & Corn-belt \\
\hline Missouri & Missouri CRD 60 & 29060 & St. Charles & 29183 & Corn-belt \\
\hline Missouri & Missouri CRD 40 & 29040 & St. Clair & 29185 & Corn-belt \\
\hline Missouri & Missouri CRD 60 & 29060 & Ste. Genevieve & 29186 & Corn-belt \\
\hline Missouri & Missouri CRD 60 & 29060 & St. Francois & 29187 & Corn-belt \\
\hline Missouri & Missouri CRD 60 & 29060 & St. Louis & 29189 & Corn-belt \\
\hline Missouri & Missouri CRD 50 & 29050 & Saline & 29195 & Corn-belt \\
\hline Missouri & Missouri CRD 20 & 29020 & Schuyler & 29197 & Corn-belt \\
\hline Missouri & Missouri CRD 30 & 29030 & Scotland & 29199 & Corn-belt \\
\hline Missouri & Missouri CRD 90 & 29090 & Scott & 29201 & Corn-belt \\
\hline Missouri & Missouri CRD 80 & 29080 & Shannon & 29203 & Corn-belt \\
\hline Missouri & Missouri CRD 30 & 29030 & Shelby & 29205 & Corn-belt \\
\hline Missouri & Missouri CRD 90 & 29090 & Stoddard & 29207 & Corn-belt \\
\hline Missouri & Missouri CRD 70 & 29070 & Stone & 29209 & Corn-belt \\
\hline Missouri & Missouri CRD 20 & 29020 & Sullivan & 29211 & Corn-belt \\
\hline Missouri & Missouri CRD 80 & 29080 & Taney & 29213 & Corn-belt \\
\hline
\end{tabular}


Table 1. Crop Reporting Districts (CRD) of the conterminous United States and associated counties.-Continued

[FIPS, Federal Information Processing Standard; USDA, U.S. Department of Agriculture]

\begin{tabular}{|c|c|c|c|c|c|}
\hline State & $\begin{array}{l}\text { Crop Reporting District } \\
\text { (CRD) }\end{array}$ & CRD Identifier & County Name & County FIPS & $\begin{array}{c}\text { USDA Farm Production } \\
\text { Region }\end{array}$ \\
\hline Missouri & Missouri CRD 80 & 29080 & Texas & 29215 & Corn-belt \\
\hline Missouri & Missouri CRD 40 & 29040 & Vernon & 29217 & Corn-belt \\
\hline Missouri & Missouri CRD 60 & 29060 & Warren & 29219 & Corn-belt \\
\hline Missouri & Missouri CRD 60 & 29060 & Washington & 29221 & Corn-belt \\
\hline Missouri & Missouri CRD 80 & 29080 & Wayne & 29223 & Corn-belt \\
\hline Missouri & Missouri CRD 80 & 29080 & Webster & 29225 & Corn-belt \\
\hline Missouri & Missouri CRD 10 & 29010 & Worth & 29227 & Corn-belt \\
\hline Missouri & Missouri CRD 80 & 29080 & Wright & 29229 & Corn-belt \\
\hline Missouri & Missouri CRD 60 & 29060 & St. Louis & 29510 & Corn-belt \\
\hline Montana & Montana CRD 70 & 30070 & Beaverhead & 30001 & Mountain \\
\hline Montana & Montana CRD 80 & 30080 & Big Horn & 30003 & Mountain \\
\hline Montana & Montana CRD 20 & 30020 & Blaine & 30005 & Mountain \\
\hline Montana & Montana CRD 50 & 30050 & Broadwater & 30007 & Mountain \\
\hline Montana & Montana CRD 80 & 30080 & Carbon & 30009 & Mountain \\
\hline Montana & Montana CRD 90 & 30090 & Carter & 30011 & Mountain \\
\hline Montana & Montana CRD 50 & 30050 & Cascade & 30013 & Mountain \\
\hline Montana & Montana CRD 20 & 30020 & Chouteau & 30015 & Mountain \\
\hline Montana & Montana CRD 90 & 30090 & Custer & 30017 & Mountain \\
\hline Montana & Montana CRD 30 & 30030 & Daniels & 30019 & Mountain \\
\hline Montana & Montana CRD 30 & 30030 & Dawson & 30021 & Mountain \\
\hline Montana & Montana CRD 10 & 30010 & Deer Lodge & 30023 & Mountain \\
\hline Montana & Montana CRD 90 & 30090 & Fallon & 30025 & Mountain \\
\hline Montana & Montana CRD 50 & 30050 & Fergus & 30027 & Mountain \\
\hline Montana & Montana CRD 10 & 30010 & Flathead & 30029 & Mountain \\
\hline Montana & Montana CRD 70 & 30070 & Gallatin & 30031 & Mountain \\
\hline Montana & Montana CRD 30 & 30030 & Garfield & 30033 & Mountain \\
\hline Montana & Montana CRD 20 & 30020 & Glacier & 30035 & Mountain \\
\hline Montana & Montana CRD 50 & 30050 & Golden Valley & 30037 & Mountain \\
\hline Montana & Montana CRD 10 & 30010 & Granite & 30039 & Mountain \\
\hline Montana & Montana CRD 20 & 30020 & Hill & 30041 & Mountain \\
\hline Montana & Montana CRD 70 & 30070 & Jefferson & 30043 & Mountain \\
\hline Montana & Montana CRD 50 & 30050 & Judith Basin & 30045 & Mountain \\
\hline Montana & Montana CRD 10 & 30010 & Lake & 30047 & Mountain \\
\hline Montana & Montana CRD 50 & 30050 & Lewis and Clark & 30049 & Mountain \\
\hline Montana & Montana CRD 20 & 30020 & Liberty & 30051 & Mountain \\
\hline Montana & Montana CRD 10 & 30010 & Lincoln & 30053 & Mountain \\
\hline Montana & Montana CRD 30 & 30030 & McCone & 30055 & Mountain \\
\hline Montana & Montana CRD 70 & 30070 & Madison & 30057 & Mountain \\
\hline Montana & Montana CRD 50 & 30050 & Meagher & 30059 & Mountain \\
\hline Montana & Montana CRD 10 & 30010 & Mineral & 30061 & Mountain \\
\hline Montana & Montana CRD 10 & 30010 & Missoula & 30063 & Mountain \\
\hline Montana & Montana CRD 50 & 30050 & Musselshell & 30065 & Mountain \\
\hline
\end{tabular}


Table 1. Crop Reporting Districts (CRD) of the conterminous United States and associated counties.-Continued

[FIPS, Federal Information Processing Standard; USDA, U.S. Department of Agriculture]

\begin{tabular}{|c|c|c|c|c|c|}
\hline State & $\begin{array}{l}\text { Crop Reporting District } \\
\text { (CRD) }\end{array}$ & CRD Identifier & County Name & County FIPS & $\begin{array}{l}\text { USDA Farm Production } \\
\text { Region }\end{array}$ \\
\hline Montana & Montana CRD 80 & 30080 & Park & 30067 & Mountain \\
\hline Montana & Montana CRD 50 & 30050 & Petroleum & 30069 & Mountain \\
\hline Montana & Montana CRD 20 & 30020 & Phillips & 30071 & Mountain \\
\hline Montana & Montana CRD 20 & 30020 & Pondera & 30073 & Mountain \\
\hline Montana & Montana CRD 90 & 30090 & Powder River & 30075 & Mountain \\
\hline Montana & Montana CRD 10 & 30010 & Powell & 30077 & Mountain \\
\hline Montana & Montana CRD 90 & 30090 & Prairie & 30079 & Mountain \\
\hline Montana & Montana CRD 10 & 30010 & Ravalli & 30081 & Mountain \\
\hline Montana & Montana CRD 30 & 30030 & Richland & 30083 & Mountain \\
\hline Montana & Montana CRD 30 & 30030 & Roosevelt & 30085 & Mountain \\
\hline Montana & Montana CRD 90 & 30090 & Rosebud & 30087 & Mountain \\
\hline Montana & Montana CRD 10 & 30010 & Sanders & 30089 & Mountain \\
\hline Montana & Montana CRD 30 & 30030 & Sheridan & 30091 & Mountain \\
\hline Montana & Montana CRD 70 & 30070 & Silver Bow & 30093 & Mountain \\
\hline Montana & Montana CRD 80 & 30080 & Stillwater & 30095 & Mountain \\
\hline Montana & Montana CRD 80 & 30080 & Sweet Grass & 30097 & Mountain \\
\hline Montana & Montana CRD 20 & 30020 & Teton & 30099 & Mountain \\
\hline Montana & Montana CRD 20 & 30020 & Toole & 30101 & Mountain \\
\hline Montana & Montana CRD 80 & 30080 & Treasure & 30103 & Mountain \\
\hline Montana & Montana CRD 30 & 30030 & Valley & 30105 & Mountain \\
\hline Montana & Montana CRD 50 & 30050 & Wheatland & 30107 & Mountain \\
\hline Montana & Montana CRD 90 & 30090 & Wibaux & 30109 & Mountain \\
\hline Montana & Montana CRD 80 & 30080 & Yellowstone & 30111 & Mountain \\
\hline Nebraska & Nebraska CRD 80 & 31080 & Adams & 31001 & Northern-plains \\
\hline Nebraska & Nebraska CRD 30 & 31030 & Antelope & 31003 & Northern-plains \\
\hline Nebraska & Nebraska CRD 20 & 31020 & Arthur & 31005 & Northern-plains \\
\hline Nebraska & Nebraska CRD 10 & 31010 & Banner & 31007 & Northern-plains \\
\hline Nebraska & Nebraska CRD 20 & 31020 & Blaine & 31009 & Northern-plains \\
\hline Nebraska & Nebraska CRD 30 & 31030 & Boone & 31011 & Northern-plains \\
\hline Nebraska & Nebraska CRD 10 & 31010 & Box Butte & 31013 & Northern-plains \\
\hline Nebraska & Nebraska CRD 20 & 31020 & Boyd & 31015 & Northern-plains \\
\hline Nebraska & Nebraska CRD 20 & 31020 & Brown & 31017 & Northern-plains \\
\hline Nebraska & Nebraska CRD 50 & 31050 & Buffalo & 31019 & Northern-plains \\
\hline Nebraska & Nebraska CRD 30 & 31030 & Burt & 31021 & Northern-plains \\
\hline Nebraska & Nebraska CRD 60 & 31060 & Butler & 31023 & Northern-plains \\
\hline Nebraska & Nebraska CRD 60 & 31060 & Cass & 31025 & Northern-plains \\
\hline Nebraska & Nebraska CRD 30 & 31030 & Cedar & 31027 & Northern-plains \\
\hline Nebraska & Nebraska CRD 70 & 31070 & Chase & 31029 & Northern-plains \\
\hline Nebraska & Nebraska CRD 20 & 31020 & Cherry & 31031 & Northern-plains \\
\hline Nebraska & Nebraska CRD 10 & 31010 & Cheyenne & 31033 & Northern-plains \\
\hline Nebraska & Nebraska CRD 90 & 31090 & Clay & 31035 & Northern-plains \\
\hline Nebraska & Nebraska CRD 60 & 31060 & Colfax & 31037 & Northern-plains \\
\hline
\end{tabular}


Table 1. Crop Reporting Districts (CRD) of the conterminous United States and associated counties.-Continued

[FIPS, Federal Information Processing Standard; USDA, U.S. Department of Agriculture]

\begin{tabular}{|c|c|c|c|c|c|}
\hline State & $\begin{array}{l}\text { Crop Reporting District } \\
\text { (CRD) }\end{array}$ & CRD Identifier & County Name & County FIPS & $\begin{array}{c}\text { USDA Farm Production } \\
\text { Region }\end{array}$ \\
\hline Nebraska & Nebraska CRD 30 & 31030 & Cuming & 31039 & Northern-plains \\
\hline Nebraska & Nebraska CRD 50 & 31050 & Custer & 31041 & Northern-plains \\
\hline Nebraska & Nebraska CRD 30 & 31030 & Dakota & 31043 & Northern-plains \\
\hline Nebraska & Nebraska CRD 10 & 31010 & Dawes & 31045 & Northern-plains \\
\hline Nebraska & Nebraska CRD 50 & 31050 & Dawson & 31047 & Northern-plains \\
\hline Nebraska & Nebraska CRD 10 & 31010 & Deuel & 31049 & Northern-plains \\
\hline Nebraska & Nebraska CRD 30 & 31030 & Dixon & 31051 & Northern-plains \\
\hline Nebraska & Nebraska CRD 60 & 31060 & Dodge & 31053 & Northern-plains \\
\hline Nebraska & Nebraska CRD 60 & 31060 & Douglas & 31055 & Northern-plains \\
\hline Nebraska & Nebraska CRD 70 & 31070 & Dundy & 31057 & Northern-plains \\
\hline Nebraska & Nebraska CRD 90 & 31090 & Fillmore & 31059 & Northern-plains \\
\hline Nebraska & Nebraska CRD 80 & 31080 & Franklin & 31061 & Northern-plains \\
\hline Nebraska & Nebraska CRD 70 & 31070 & Frontier & 31063 & Northern-plains \\
\hline Nebraska & Nebraska CRD 80 & 31080 & Furnas & 31065 & Northern-plains \\
\hline Nebraska & Nebraska CRD 90 & 31090 & Gage & 31067 & Northern-plains \\
\hline Nebraska & Nebraska CRD 10 & 31010 & Garden & 31069 & Northern-plains \\
\hline Nebraska & Nebraska CRD 20 & 31020 & Garfield & 31071 & Northern-plains \\
\hline Nebraska & Nebraska CRD 80 & 31080 & Gosper & 31073 & Northern-plains \\
\hline Nebraska & Nebraska CRD 20 & 31020 & Grant & 31075 & Northern-plains \\
\hline Nebraska & Nebraska CRD 50 & 31050 & Greeley & 31077 & Northern-plains \\
\hline Nebraska & Nebraska CRD 50 & 31050 & Hall & 31079 & Northern-plains \\
\hline Nebraska & Nebraska CRD 60 & 31060 & Hamilton & 31081 & Northern-plains \\
\hline Nebraska & Nebraska CRD 80 & 31080 & Harlan & 31083 & Northern-plains \\
\hline Nebraska & Nebraska CRD 70 & 31070 & Hayes & 31085 & Northern-plains \\
\hline Nebraska & Nebraska CRD 70 & 31070 & Hitchcock & 31087 & Northern-plains \\
\hline Nebraska & Nebraska CRD 20 & 31020 & Holt & 31089 & Northern-plains \\
\hline Nebraska & Nebraska CRD 20 & 31020 & Hooker & 31091 & Northern-plains \\
\hline Nebraska & Nebraska CRD 50 & 31050 & Howard & 31093 & Northern-plains \\
\hline Nebraska & Nebraska CRD 90 & 31090 & Jefferson & 31095 & Northern-plains \\
\hline Nebraska & Nebraska CRD 90 & 31090 & Johnson & 31097 & Northern-plains \\
\hline Nebraska & Nebraska CRD 80 & 31080 & Kearney & 31099 & Northern-plains \\
\hline Nebraska & Nebraska CRD 70 & 31070 & Keith & 31101 & Northern-plains \\
\hline Nebraska & Nebraska CRD 20 & 31020 & Keya Paha & 31103 & Northern-plains \\
\hline Nebraska & Nebraska CRD 10 & 31010 & Kimball & 31105 & Northern-plains \\
\hline Nebraska & Nebraska CRD 30 & 31030 & Knox & 31107 & Northern-plains \\
\hline Nebraska & Nebraska CRD 60 & 31060 & Lancaster & 31109 & Northern-plains \\
\hline Nebraska & Nebraska CRD 70 & 31070 & Lincoln & 31111 & Northern-plains \\
\hline Nebraska & Nebraska CRD 20 & 31020 & Logan & 31113 & Northern-plains \\
\hline Nebraska & Nebraska CRD 20 & 31020 & Loup & 31115 & Northern-plains \\
\hline Nebraska & Nebraska CRD 20 & 31020 & McPherson & 31117 & Northern-plains \\
\hline Nebraska & Nebraska CRD 30 & 31030 & Madison & 31119 & Northern-plains \\
\hline Nebraska & Nebraska CRD 60 & 31060 & Merrick & 31121 & Northern-plains \\
\hline
\end{tabular}


Table 1. Crop Reporting Districts (CRD) of the conterminous United States and associated counties.-Continued

[FIPS, Federal Information Processing Standard; USDA, U.S. Department of Agriculture]

\begin{tabular}{|c|c|c|c|c|c|}
\hline State & $\begin{array}{l}\text { Crop Reporting District } \\
\text { (CRD) }\end{array}$ & CRD Identifier & County Name & County FIPS & $\begin{array}{c}\text { USDA Farm Production } \\
\text { Region }\end{array}$ \\
\hline Nebraska & Nebraska CRD 10 & 31010 & Morrill & 31123 & Northern-plains \\
\hline Nebraska & Nebraska CRD 60 & 31060 & Nance & 31125 & Northern-plains \\
\hline Nebraska & Nebraska CRD 90 & 31090 & Nemaha & 31127 & Northern-plains \\
\hline Nebraska & Nebraska CRD 90 & 31090 & Nuckolls & 31129 & Northern-plains \\
\hline Nebraska & Nebraska CRD 90 & 31090 & Otoe & 31131 & Northern-plains \\
\hline Nebraska & Nebraska CRD 90 & 31090 & Pawnee & 31133 & Northern-plains \\
\hline Nebraska & Nebraska CRD 70 & 31070 & Perkins & 31135 & Northern-plains \\
\hline Nebraska & Nebraska CRD 80 & 31080 & Phelps & 31137 & Northern-plains \\
\hline Nebraska & Nebraska CRD 30 & 31030 & Pierce & 31139 & Northern-plains \\
\hline Nebraska & Nebraska CRD 60 & 31060 & Platte & 31141 & Northern-plains \\
\hline Nebraska & Nebraska CRD 60 & 31060 & Polk & 31143 & Northern-plains \\
\hline Nebraska & Nebraska CRD 70 & 31070 & Red Willow & 31145 & Northern-plains \\
\hline Nebraska & Nebraska CRD 90 & 31090 & Richardson & 31147 & Northern-plains \\
\hline Nebraska & Nebraska CRD 20 & 31020 & Rock & 31149 & Northern-plains \\
\hline Nebraska & Nebraska CRD 90 & 31090 & Saline & 31151 & Northern-plains \\
\hline Nebraska & Nebraska CRD 60 & 31060 & Sarpy & 31153 & Northern-plains \\
\hline Nebraska & Nebraska CRD 60 & 31060 & Saunders & 31155 & Northern-plains \\
\hline Nebraska & Nebraska CRD 10 & 31010 & Scotts Bluff & 31157 & Northern-plains \\
\hline Nebraska & Nebraska CRD 60 & 31060 & Seward & 31159 & Northern-plains \\
\hline Nebraska & Nebraska CRD 10 & 31010 & Sheridan & 31161 & Northern-plains \\
\hline Nebraska & Nebraska CRD 50 & 31050 & Sherman & 31163 & Northern-plains \\
\hline Nebraska & Nebraska CRD 10 & 31010 & Sioux & 31165 & Northern-plains \\
\hline Nebraska & Nebraska CRD 30 & 31030 & Stanton & 31167 & Northern-plains \\
\hline Nebraska & Nebraska CRD 90 & 31090 & Thayer & 31169 & Northern-plains \\
\hline Nebraska & Nebraska CRD 20 & 31020 & Thomas & 31171 & Northern-plains \\
\hline Nebraska & Nebraska CRD 30 & 31030 & Thurston & 31173 & Northern-plains \\
\hline Nebraska & Nebraska CRD 50 & 31050 & Valley & 31175 & Northern-plains \\
\hline Nebraska & Nebraska CRD 60 & 31060 & Washington & 31177 & Northern-plains \\
\hline Nebraska & Nebraska CRD 30 & 31030 & Wayne & 31179 & Northern-plains \\
\hline Nebraska & Nebraska CRD 80 & 31080 & Webster & 31181 & Northern-plains \\
\hline Nebraska & Nebraska CRD 20 & 31020 & Wheeler & 31183 & Northern-plains \\
\hline Nebraska & Nebraska CRD 60 & 31060 & York & 31185 & Northern-plains \\
\hline Nevada & Nevada CRD 10 & 32010 & Churchill & 32001 & Mountain \\
\hline Nevada & Nevada CRD 80 & 32080 & Clark & 32003 & Mountain \\
\hline Nevada & Nevada CRD 10 & 32010 & Douglas & 32005 & Mountain \\
\hline Nevada & Nevada CRD 30 & 32030 & Elko & 32007 & Mountain \\
\hline Nevada & Nevada CRD 80 & 32080 & Esmeralda & 32009 & Mountain \\
\hline Nevada & Nevada CRD 30 & 32030 & Eureka & 32011 & Mountain \\
\hline Nevada & Nevada CRD 10 & 32010 & Humboldt & 32013 & Mountain \\
\hline Nevada & Nevada CRD 30 & 32030 & Lander & 32015 & Mountain \\
\hline Nevada & Nevada CRD 80 & 32080 & Lincoln & 32017 & Mountain \\
\hline Nevada & Nevada CRD 10 & 32010 & Lyon & 32019 & Mountain \\
\hline
\end{tabular}


Table 1. Crop Reporting Districts (CRD) of the conterminous United States and associated counties.-Continued

[FIPS, Federal Information Processing Standard; USDA, U.S. Department of Agriculture]

\begin{tabular}{|c|c|c|c|c|c|}
\hline State & $\begin{array}{l}\text { Crop Reporting District } \\
\text { (CRD) }\end{array}$ & CRD Identifier & County Name & County FIPS & $\begin{array}{l}\text { USDA Farm Production } \\
\text { Region }\end{array}$ \\
\hline Nevada & Nevada CRD 80 & 32080 & Mineral & 32021 & Mountain \\
\hline Nevada & Nevada CRD 80 & 32080 & Nye & 32023 & Mountain \\
\hline Nevada & Nevada CRD 10 & 32010 & Pershing & 32027 & Mountain \\
\hline Nevada & Nevada CRD 10 & 32010 & Storey & 32029 & Mountain \\
\hline Nevada & Nevada CRD 10 & 32010 & Washoe & 32031 & Mountain \\
\hline Nevada & Nevada CRD 30 & 32030 & White Pine & 32033 & Mountain \\
\hline Nevada & Nevada CRD 10 & 32010 & Carson City & 32510 & Mountain \\
\hline New Hampshire & New Hampshire CRD 10 & 33010 & Belknap & 33001 & Northeast \\
\hline New Hampshire & New Hampshire CRD 10 & 33010 & Carroll & 33003 & Northeast \\
\hline New Hampshire & New Hampshire CRD 10 & 33010 & Cheshire & 33005 & Northeast \\
\hline New Hampshire & New Hampshire CRD 10 & 33010 & Coos & 33007 & Northeast \\
\hline New Hampshire & New Hampshire CRD 10 & 33010 & Grafton & 33009 & Northeast \\
\hline New Hampshire & New Hampshire CRD 10 & 33010 & Hillsborough & 33011 & Northeast \\
\hline New Hampshire & New Hampshire CRD 10 & 33010 & Merrimack & 33013 & Northeast \\
\hline New Hampshire & New Hampshire CRD 10 & 33010 & Rockingham & 33015 & Northeast \\
\hline New Hampshire & New Hampshire CRD 10 & 33010 & Strafford & 33017 & Northeast \\
\hline New Hampshire & New Hampshire CRD 10 & 33010 & Sullivan & 33019 & Northeast \\
\hline New Jersey & New Jersey CRD 80 & 34080 & Atlantic & 34001 & Northeast \\
\hline New Jersey & New Jersey CRD 20 & 34020 & Bergen & 34003 & Northeast \\
\hline New Jersey & New Jersey CRD 50 & 34050 & Burlington & 34005 & Northeast \\
\hline New Jersey & New Jersey CRD 80 & 34080 & Camden & 34007 & Northeast \\
\hline New Jersey & New Jersey CRD 80 & 34080 & Cape May & 34009 & Northeast \\
\hline New Jersey & New Jersey CRD 80 & 34080 & Cumberland & 34011 & Northeast \\
\hline New Jersey & New Jersey CRD 20 & 34020 & Essex & 34013 & Northeast \\
\hline New Jersey & New Jersey CRD 80 & 34080 & Gloucester & 34015 & Northeast \\
\hline New Jersey & New Jersey CRD 20 & 34020 & Hudson & 34017 & Northeast \\
\hline New Jersey & New Jersey CRD 20 & 34020 & Hunterdon & 34019 & Northeast \\
\hline New Jersey & New Jersey CRD 50 & 34050 & Mercer & 34021 & Northeast \\
\hline New Jersey & New Jersey CRD 50 & 34050 & Middlesex & 34023 & Northeast \\
\hline New Jersey & New Jersey CRD 50 & 34050 & Monmouth & 34025 & Northeast \\
\hline New Jersey & New Jersey CRD 20 & 34020 & Morris & 34027 & Northeast \\
\hline New Jersey & New Jersey CRD 50 & 34050 & Ocean & 34029 & Northeast \\
\hline New Jersey & New Jersey CRD 20 & 34020 & Passaic & 34031 & Northeast \\
\hline New Jersey & New Jersey CRD 80 & 34080 & Salem & 34033 & Northeast \\
\hline New Jersey & New Jersey CRD 20 & 34020 & Somerset & 34035 & Northeast \\
\hline New Jersey & New Jersey CRD 20 & 34020 & Sussex & 34037 & Northeast \\
\hline New Jersey & New Jersey CRD 20 & 34020 & Union & 34039 & Northeast \\
\hline New Jersey & New Jersey CRD 20 & 34020 & Warren & 34041 & Northeast \\
\hline New Mexico & New Mexico CRD 10 & 35010 & Bernalillo & 35001 & Mountain \\
\hline New Mexico & New Mexico CRD 70 & 35070 & Catron & 35003 & Mountain \\
\hline New Mexico & New Mexico CRD 90 & 35090 & Chaves & 35005 & Mountain \\
\hline New Mexico & New Mexico CRD 10 & 35010 & Cibola & 35006 & Mountain \\
\hline
\end{tabular}


72 Method for Estimating Annual Atrazine Use for Counties in the Conterminous United States, 1992-2007

Table 1. Crop Reporting Districts (CRD) of the conterminous United States and associated counties.-Continued

[FIPS, Federal Information Processing Standard; USDA, U.S. Department of Agriculture]

\begin{tabular}{|c|c|c|c|c|c|}
\hline State & $\begin{array}{l}\text { Crop Reporting District } \\
\text { (CRD) }\end{array}$ & CRD Identifier & County Name & County FIPS & $\begin{array}{l}\text { USDA Farm Production } \\
\text { Region }\end{array}$ \\
\hline New Mexico & New Mexico CRD 30 & 35030 & Colfax & 35007 & Mountain \\
\hline New Mexico & New Mexico CRD 30 & 35030 & Curry & 35009 & Mountain \\
\hline New Mexico & New Mexico CRD 30 & 35030 & De Baca & 35011 & Mountain \\
\hline New Mexico & New Mexico CRD 90 & 35090 & Dona Ana & 35013 & Mountain \\
\hline New Mexico & New Mexico CRD 90 & 35090 & Eddy & 35015 & Mountain \\
\hline New Mexico & New Mexico CRD 70 & 35070 & Grant & 35017 & Mountain \\
\hline New Mexico & New Mexico CRD 30 & 35030 & Guadalupe & 35019 & Mountain \\
\hline New Mexico & New Mexico CRD 30 & 35030 & Harding & 35021 & Mountain \\
\hline New Mexico & New Mexico CRD 70 & 35070 & Hidalgo & 35023 & Mountain \\
\hline New Mexico & New Mexico CRD 90 & 35090 & Lea & 35025 & Mountain \\
\hline New Mexico & New Mexico CRD 90 & 35090 & Lincoln & 35027 & Mountain \\
\hline New Mexico & New Mexico CRD 10 & 35010 & Los Alamos & 35028 & Mountain \\
\hline New Mexico & New Mexico CRD 70 & 35070 & Luna & 35029 & Mountain \\
\hline New Mexico & New Mexico CRD 10 & 35010 & McKinley & 35031 & Mountain \\
\hline New Mexico & New Mexico CRD 30 & 35030 & Mora & 35033 & Mountain \\
\hline New Mexico & New Mexico CRD 90 & 35090 & Otero & 35035 & Mountain \\
\hline New Mexico & New Mexico CRD 30 & 35030 & Quay & 35037 & Mountain \\
\hline New Mexico & New Mexico CRD 10 & 35010 & Rio Arriba & 35039 & Mountain \\
\hline New Mexico & New Mexico CRD 30 & 35030 & Roosevelt & 35041 & Mountain \\
\hline New Mexico & New Mexico CRD 10 & 35010 & Sandoval & 35043 & Mountain \\
\hline New Mexico & New Mexico CRD 10 & 35010 & San Juan & 35045 & Mountain \\
\hline New Mexico & New Mexico CRD 30 & 35030 & San Miguel & 35047 & Mountain \\
\hline New Mexico & New Mexico CRD 10 & 35010 & Santa Fe & 35049 & Mountain \\
\hline New Mexico & New Mexico CRD 70 & 35070 & Sierra & 35051 & Mountain \\
\hline New Mexico & New Mexico CRD 70 & 35070 & Socorro & 35053 & Mountain \\
\hline New Mexico & New Mexico CRD 10 & 35010 & Taos & 35055 & Mountain \\
\hline New Mexico & New Mexico CRD 30 & 35030 & Torrance & 35057 & Mountain \\
\hline New Mexico & New Mexico CRD 30 & 35030 & Union & 35059 & Mountain \\
\hline New Mexico & New Mexico CRD 10 & 35010 & Valencia & 35061 & Mountain \\
\hline New York & New York CRD 60 & 36060 & Albany & 36001 & Northeast \\
\hline New York & New York CRD 70 & 36070 & Allegany & 36003 & Northeast \\
\hline New York & New York CRD 91 & 36091 & Bronx & 36005 & Northeast \\
\hline New York & New York CRD 80 & 36080 & Broome & 36007 & Northeast \\
\hline New York & New York CRD 70 & 36070 & Cattaraugus & 36009 & Northeast \\
\hline New York & New York CRD 50 & 36050 & Cayuga & 36011 & Northeast \\
\hline New York & New York CRD 70 & 36070 & Chautauqua & 36013 & Northeast \\
\hline New York & New York CRD 80 & 36080 & Chemung & 36015 & Northeast \\
\hline New York & New York CRD 50 & 36050 & Chenango & 36017 & Northeast \\
\hline New York & New York CRD 30 & 36030 & Clinton & 36019 & Northeast \\
\hline New York & New York CRD 90 & 36090 & Columbia & 36021 & Northeast \\
\hline New York & New York CRD 50 & 36050 & Cortland & 36023 & Northeast \\
\hline New York & New York CRD 90 & 36090 & Delaware & 36025 & Northeast \\
\hline
\end{tabular}


Table 1. Crop Reporting Districts (CRD) of the conterminous United States and associated counties.-Continued

[FIPS, Federal Information Processing Standard; USDA, U.S. Department of Agriculture]

\begin{tabular}{|c|c|c|c|c|c|}
\hline State & $\begin{array}{l}\text { Crop Reporting District } \\
\text { (CRD) }\end{array}$ & CRD Identifier & County Name & County FIPS & $\begin{array}{c}\text { USDA Farm Production } \\
\text { Region }\end{array}$ \\
\hline New York & New York CRD 90 & 36090 & Dutchess & 36027 & Northeast \\
\hline New York & New York CRD 40 & 36040 & Erie & 36029 & Northeast \\
\hline New York & New York CRD 30 & 36030 & Essex & 36031 & Northeast \\
\hline New York & New York CRD 30 & 36030 & Franklin & 36033 & Northeast \\
\hline New York & New York CRD 60 & 36060 & Fulton & 36035 & Northeast \\
\hline New York & New York CRD 40 & 36040 & Genesee & 36037 & Northeast \\
\hline New York & New York CRD 90 & 36090 & Greene & 36039 & Northeast \\
\hline New York & New York CRD 30 & 36030 & Hamilton & 36041 & Northeast \\
\hline New York & New York CRD 50 & 36050 & Herkimer & 36043 & Northeast \\
\hline New York & New York CRD 20 & 36020 & Jefferson & 36045 & Northeast \\
\hline New York & New York CRD 91 & 36091 & Kings & 36047 & Northeast \\
\hline New York & New York CRD 20 & 36020 & Lewis & 36049 & Northeast \\
\hline New York & New York CRD 40 & 36040 & Livingston & 36051 & Northeast \\
\hline New York & New York CRD 50 & 36050 & Madison & 36053 & Northeast \\
\hline New York & New York CRD 40 & 36040 & Monroe & 36055 & Northeast \\
\hline New York & New York CRD 60 & 36060 & Montgomery & 36057 & Northeast \\
\hline New York & New York CRD 91 & 36091 & Nassau & 36059 & Northeast \\
\hline New York & New York CRD 91 & 36091 & New York & 36061 & Northeast \\
\hline New York & New York CRD 40 & 36040 & Niagara & 36063 & Northeast \\
\hline New York & New York CRD 50 & 36050 & Oneida & 36065 & Northeast \\
\hline New York & New York CRD 50 & 36050 & Onondaga & 36067 & Northeast \\
\hline New York & New York CRD 40 & 36040 & Ontario & 36069 & Northeast \\
\hline New York & New York CRD 90 & 36090 & Orange & 36071 & Northeast \\
\hline New York & New York CRD 40 & 36040 & Orleans & 36073 & Northeast \\
\hline New York & New York CRD 50 & 36050 & Oswego & 36075 & Northeast \\
\hline New York & New York CRD 50 & 36050 & Otsego & 36077 & Northeast \\
\hline New York & New York CRD 90 & 36090 & Putnam & 36079 & Northeast \\
\hline New York & New York CRD 91 & 36091 & Queens & 36081 & Northeast \\
\hline New York & New York CRD 60 & 36060 & Rensselaer & 36083 & Northeast \\
\hline New York & New York CRD 91 & 36091 & Richmond & 36085 & Northeast \\
\hline New York & New York CRD 90 & 36090 & Rockland & 36087 & Northeast \\
\hline New York & New York CRD 20 & 36020 & St. Lawrence & 36089 & Northeast \\
\hline New York & New York CRD 60 & 36060 & Saratoga & 36091 & Northeast \\
\hline New York & New York CRD 60 & 36060 & Schenectady & 36093 & Northeast \\
\hline New York & New York CRD 60 & 36060 & Schoharie & 36095 & Northeast \\
\hline New York & New York CRD 80 & 36080 & Schuyler & 36097 & Northeast \\
\hline New York & New York CRD 40 & 36040 & Seneca & 36099 & Northeast \\
\hline New York & New York CRD 70 & 36070 & Steuben & 36101 & Northeast \\
\hline New York & New York CRD 91 & 36091 & Suffolk & 36103 & Northeast \\
\hline New York & New York CRD 90 & 36090 & Sullivan & 36105 & Northeast \\
\hline New York & New York CRD 80 & 36080 & Tioga & 36107 & Northeast \\
\hline New York & New York CRD 80 & 36080 & Tompkins & 36109 & Northeast \\
\hline
\end{tabular}


Table 1. Crop Reporting Districts (CRD) of the conterminous United States and associated counties. - Continued

[FIPS, Federal Information Processing Standard; USDA, U.S. Department of Agriculture]

\begin{tabular}{|c|c|c|c|c|c|}
\hline State & $\begin{array}{l}\text { Crop Reporting District } \\
\text { (CRD) }\end{array}$ & CRD Identifier & County Name & County FIPS & $\begin{array}{c}\text { USDA Farm Production } \\
\text { Region }\end{array}$ \\
\hline New York & New York CRD 90 & 36090 & Ulster & 36111 & Northeast \\
\hline New York & New York CRD 30 & 36030 & Warren & 36113 & Northeast \\
\hline New York & New York CRD 60 & 36060 & Washington & 36115 & Northeast \\
\hline New York & New York CRD 40 & 36040 & Wayne & 36117 & Northeast \\
\hline New York & New York CRD 90 & 36090 & Westchester & 36119 & Northeast \\
\hline New York & New York CRD 40 & 36040 & Wyoming & 36121 & Northeast \\
\hline New York & New York CRD 40 & 36040 & Yates & 36123 & Northeast \\
\hline North Carolina & North Carolina CRD 40 & 37040 & Alamance & 37001 & Appalachian \\
\hline North Carolina & North Carolina CRD 50 & 37050 & Alexander & 37003 & Appalachian \\
\hline North Carolina & North Carolina CRD 10 & 37010 & Alleghany & 37005 & Appalachian \\
\hline North Carolina & North Carolina CRD 60 & 37060 & Anson & 37007 & Appalachian \\
\hline North Carolina & North Carolina CRD 10 & 37010 & Ashe & 37009 & Appalachian \\
\hline North Carolina & North Carolina CRD 10 & 37010 & Avery & 37011 & Appalachian \\
\hline North Carolina & North Carolina CRD 80 & 37080 & Beaufort & 37013 & Appalachian \\
\hline North Carolina & North Carolina CRD 70 & 37070 & Bertie & 37015 & Appalachian \\
\hline North Carolina & North Carolina CRD 90 & 37090 & Bladen & 37017 & Appalachian \\
\hline North Carolina & North Carolina CRD 90 & 37090 & Brunswick & 37019 & Appalachian \\
\hline North Carolina & North Carolina CRD 20 & 37020 & Buncombe & 37021 & Appalachian \\
\hline North Carolina & North Carolina CRD 20 & 37020 & Burke & 37023 & Appalachian \\
\hline North Carolina & North Carolina CRD 60 & 37060 & Cabarrus & 37025 & Appalachian \\
\hline North Carolina & North Carolina CRD 10 & 37010 & Caldwell & 37027 & Appalachian \\
\hline North Carolina & North Carolina CRD 70 & 37070 & Camden & 37029 & Appalachian \\
\hline North Carolina & North Carolina CRD 80 & 37080 & Carteret & 37031 & Appalachian \\
\hline North Carolina & North Carolina CRD 40 & 37040 & Caswell & 37033 & Appalachian \\
\hline North Carolina & North Carolina CRD 50 & 37050 & Catawba & 37035 & Appalachian \\
\hline North Carolina & North Carolina CRD 50 & 37050 & Chatham & 37037 & Appalachian \\
\hline North Carolina & North Carolina CRD 20 & 37020 & Cherokee & 37039 & Appalachian \\
\hline North Carolina & North Carolina CRD 70 & 37070 & Chowan & 37041 & Appalachian \\
\hline North Carolina & North Carolina CRD 20 & 37020 & Clay & 37043 & Appalachian \\
\hline North Carolina & North Carolina CRD 60 & 37060 & Cleveland & 37045 & Appalachian \\
\hline North Carolina & North Carolina CRD 90 & 37090 & Columbus & 37047 & Appalachian \\
\hline North Carolina & North Carolina CRD 80 & 37080 & Craven & 37049 & Appalachian \\
\hline North Carolina & North Carolina CRD 90 & 37090 & Cumberland & 37051 & Appalachian \\
\hline North Carolina & North Carolina CRD 70 & 37070 & Currituck & 37053 & Appalachian \\
\hline North Carolina & North Carolina CRD 70 & 37070 & Dare & 37055 & Appalachian \\
\hline North Carolina & North Carolina CRD 50 & 37050 & Davidson & 37057 & Appalachian \\
\hline North Carolina & North Carolina CRD 50 & 37050 & Davie & 37059 & Appalachian \\
\hline North Carolina & North Carolina CRD 90 & 37090 & Duplin & 37061 & Appalachian \\
\hline North Carolina & North Carolina CRD 40 & 37040 & Durham & 37063 & Appalachian \\
\hline North Carolina & North Carolina CRD 70 & 37070 & Edgecombe & 37065 & Appalachian \\
\hline North Carolina & North Carolina CRD 40 & 37040 & Forsyth & 37067 & Appalachian \\
\hline North Carolina & North Carolina CRD 40 & 37040 & Franklin & 37069 & Appalachian \\
\hline
\end{tabular}


Table 1. Crop Reporting Districts (CRD) of the conterminous United States and associated counties.-Continued

[FIPS, Federal Information Processing Standard; USDA, U.S. Department of Agriculture]

\begin{tabular}{|c|c|c|c|c|c|}
\hline State & $\begin{array}{l}\text { Crop Reporting District } \\
\text { (CRD) }\end{array}$ & CRD Identifier & County Name & County FIPS & $\begin{array}{c}\text { USDA Farm Production } \\
\text { Region }\end{array}$ \\
\hline North Carolina & North Carolina CRD 60 & 37060 & Gaston & 37071 & Appalachian \\
\hline North Carolina & North Carolina CRD 70 & 37070 & Gates & 37073 & Appalachian \\
\hline North Carolina & North Carolina CRD 20 & 37020 & Graham & 37075 & Appalachian \\
\hline North Carolina & North Carolina CRD 40 & 37040 & Granville & 37077 & Appalachian \\
\hline North Carolina & North Carolina CRD 80 & 37080 & Greene & 37079 & Appalachian \\
\hline North Carolina & North Carolina CRD 40 & 37040 & Guilford & 37081 & Appalachian \\
\hline North Carolina & North Carolina CRD 70 & 37070 & Halifax & 37083 & Appalachian \\
\hline North Carolina & North Carolina CRD 90 & 37090 & Harnett & 37085 & Appalachian \\
\hline North Carolina & North Carolina CRD 20 & 37020 & Haywood & 37087 & Appalachian \\
\hline North Carolina & North Carolina CRD 20 & 37020 & Henderson & 37089 & Appalachian \\
\hline North Carolina & North Carolina CRD 70 & 37070 & Hertford & 37091 & Appalachian \\
\hline North Carolina & North Carolina CRD 90 & 37090 & Hoke & 37093 & Appalachian \\
\hline North Carolina & North Carolina CRD 80 & 37080 & Hyde & 37095 & Appalachian \\
\hline North Carolina & North Carolina CRD 50 & 37050 & Iredell & 37097 & Appalachian \\
\hline North Carolina & North Carolina CRD 20 & 37020 & Jackson & 37099 & Appalachian \\
\hline North Carolina & North Carolina CRD 80 & 37080 & Johnston & 37101 & Appalachian \\
\hline North Carolina & North Carolina CRD 80 & 37080 & Jones & 37103 & Appalachian \\
\hline North Carolina & North Carolina CRD 50 & 37050 & Lee & 37105 & Appalachian \\
\hline North Carolina & North Carolina CRD 80 & 37080 & Lenoir & 37107 & Appalachian \\
\hline North Carolina & North Carolina CRD 60 & 37060 & Lincoln & 37109 & Appalachian \\
\hline North Carolina & North Carolina CRD 20 & 37020 & McDowell & 37111 & Appalachian \\
\hline North Carolina & North Carolina CRD 20 & 37020 & Macon & 37113 & Appalachian \\
\hline North Carolina & North Carolina CRD 20 & 37020 & Madison & 37115 & Appalachian \\
\hline North Carolina & North Carolina CRD 70 & 37070 & Martin & 37117 & Appalachian \\
\hline North Carolina & North Carolina CRD 60 & 37060 & Mecklenburg & 37119 & Appalachian \\
\hline North Carolina & North Carolina CRD 20 & 37020 & Mitchell & 37121 & Appalachian \\
\hline North Carolina & North Carolina CRD 60 & 37060 & Montgomery & 37123 & Appalachian \\
\hline North Carolina & North Carolina CRD 60 & 37060 & Moore & 37125 & Appalachian \\
\hline North Carolina & North Carolina CRD 70 & 37070 & Nash & 37127 & Appalachian \\
\hline North Carolina & North Carolina CRD 90 & 37090 & New Hanover & 37129 & Appalachian \\
\hline North Carolina & North Carolina CRD 70 & 37070 & Northampton & 37131 & Appalachian \\
\hline North Carolina & North Carolina CRD 90 & 37090 & Onslow & 37133 & Appalachian \\
\hline North Carolina & North Carolina CRD 40 & 37040 & Orange & 37135 & Appalachian \\
\hline North Carolina & North Carolina CRD 80 & 37080 & Pamlico & 37137 & Appalachian \\
\hline North Carolina & North Carolina CRD 70 & 37070 & Pasquotank & 37139 & Appalachian \\
\hline North Carolina & North Carolina CRD 90 & 37090 & Pender & 37141 & Appalachian \\
\hline North Carolina & North Carolina CRD 70 & 37070 & Perquimans & 37143 & Appalachian \\
\hline North Carolina & North Carolina CRD 40 & 37040 & Person & 37145 & Appalachian \\
\hline North Carolina & North Carolina CRD 80 & 37080 & Pitt & 37147 & Appalachian \\
\hline North Carolina & North Carolina CRD 20 & 37020 & Polk & 37149 & Appalachian \\
\hline North Carolina & North Carolina CRD 50 & 37050 & Randolph & 37151 & Appalachian \\
\hline North Carolina & North Carolina CRD 60 & 37060 & Richmond & 37153 & Appalachian \\
\hline
\end{tabular}


Table 1. Crop Reporting Districts (CRD) of the conterminous United States and associated counties.-Continued

[FIPS, Federal Information Processing Standard; USDA, U.S. Department of Agriculture]

\begin{tabular}{|c|c|c|c|c|c|}
\hline State & $\begin{array}{l}\text { Crop Reporting District } \\
\text { (CRD) }\end{array}$ & CRD Identifier & County Name & County FIPS & $\begin{array}{c}\text { USDA Farm Production } \\
\text { Region }\end{array}$ \\
\hline North Carolina & North Carolina CRD 90 & 37090 & Robeson & 37155 & Appalachian \\
\hline North Carolina & North Carolina CRD 40 & 37040 & Rockingham & 37157 & Appalachian \\
\hline North Carolina & North Carolina CRD 50 & 37050 & Rowan & 37159 & Appalachian \\
\hline North Carolina & North Carolina CRD 20 & 37020 & Rutherford & 37161 & Appalachian \\
\hline North Carolina & North Carolina CRD 90 & 37090 & Sampson & 37163 & Appalachian \\
\hline North Carolina & North Carolina CRD 90 & 37090 & Scotland & 37165 & Appalachian \\
\hline North Carolina & North Carolina CRD 60 & 37060 & Stanly & 37167 & Appalachian \\
\hline North Carolina & North Carolina CRD 40 & 37040 & Stokes & 37169 & Appalachian \\
\hline North Carolina & North Carolina CRD 10 & 37010 & Surry & 37171 & Appalachian \\
\hline North Carolina & North Carolina CRD 20 & 37020 & Swain & 37173 & Appalachian \\
\hline North Carolina & North Carolina CRD 20 & 37020 & Transylvania & 37175 & Appalachian \\
\hline North Carolina & North Carolina CRD 70 & 37070 & Tyrrell & 37177 & Appalachian \\
\hline North Carolina & North Carolina CRD 60 & 37060 & Union & 37179 & Appalachian \\
\hline North Carolina & North Carolina CRD 40 & 37040 & Vance & 37181 & Appalachian \\
\hline North Carolina & North Carolina CRD 50 & 37050 & Wake & 37183 & Appalachian \\
\hline North Carolina & North Carolina CRD 40 & 37040 & Warren & 37185 & Appalachian \\
\hline North Carolina & North Carolina CRD 70 & 37070 & Washington & 37187 & Appalachian \\
\hline North Carolina & North Carolina CRD 10 & 37010 & Watauga & 37189 & Appalachian \\
\hline North Carolina & North Carolina CRD 80 & 37080 & Wayne & 37191 & Appalachian \\
\hline North Carolina & North Carolina CRD 10 & 37010 & Wilkes & 37193 & Appalachian \\
\hline North Carolina & North Carolina CRD 80 & 37080 & Wilson & 37195 & Appalachian \\
\hline North Carolina & North Carolina CRD 10 & 37010 & Yadkin & 37197 & Appalachian \\
\hline North Carolina & North Carolina CRD 20 & 37020 & Yancey & 37199 & Appalachian \\
\hline North Dakota & North Dakota CRD 70 & 38070 & Adams & 38001 & Northern-plains \\
\hline North Dakota & North Dakota CRD 60 & 38060 & Barnes & 38003 & Northern-plains \\
\hline North Dakota & North Dakota CRD 20 & 38020 & Benson & 38005 & Northern-plains \\
\hline North Dakota & North Dakota CRD 70 & 38070 & Billings & 38007 & Northern-plains \\
\hline North Dakota & North Dakota CRD 20 & 38020 & Bottineau & 38009 & Northern-plains \\
\hline North Dakota & North Dakota CRD 70 & 38070 & Bowman & 38011 & Northern-plains \\
\hline North Dakota & North Dakota CRD 10 & 38010 & Burke & 38013 & Northern-plains \\
\hline North Dakota & North Dakota CRD 80 & 38080 & Burleigh & 38015 & Northern-plains \\
\hline North Dakota & North Dakota CRD 60 & 38060 & Cass & 38017 & Northern-plains \\
\hline North Dakota & North Dakota CRD 30 & 38030 & Cavalier & 38019 & Northern-plains \\
\hline North Dakota & North Dakota CRD 90 & 38090 & Dickey & 38021 & Northern-plains \\
\hline North Dakota & North Dakota CRD 10 & 38010 & Divide & 38023 & Northern-plains \\
\hline North Dakota & North Dakota CRD 40 & 38040 & Dunn & 38025 & Northern-plains \\
\hline North Dakota & North Dakota CRD 50 & 38050 & Eddy & 38027 & Northern-plains \\
\hline North Dakota & North Dakota CRD 80 & 38080 & Emmons & 38029 & Northern-plains \\
\hline North Dakota & North Dakota CRD 50 & 38050 & Foster & 38031 & Northern-plains \\
\hline North Dakota & North Dakota CRD 70 & 38070 & Golden Valley & 38033 & Northern-plains \\
\hline North Dakota & North Dakota CRD 30 & 38030 & Grand Forks & 38035 & Northern-plains \\
\hline North Dakota & North Dakota CRD 80 & 38080 & Grant & 38037 & Northern-plains \\
\hline
\end{tabular}


Table 1. Crop Reporting Districts (CRD) of the conterminous United States and associated counties.-Continued

[FIPS, Federal Information Processing Standard; USDA, U.S. Department of Agriculture]

\begin{tabular}{|c|c|c|c|c|c|}
\hline State & $\begin{array}{l}\text { Crop Reporting District } \\
\text { (CRD) }\end{array}$ & CRD Identifier & County Name & County FIPS & $\begin{array}{c}\text { USDA Farm Production } \\
\text { Region }\end{array}$ \\
\hline North Dakota & North Dakota CRD 60 & 38060 & Griggs & 38039 & Northern-plains \\
\hline North Dakota & North Dakota CRD 70 & 38070 & Hettinger & 38041 & Northern-plains \\
\hline North Dakota & North Dakota CRD 50 & 38050 & Kidder & 38043 & Northern-plains \\
\hline North Dakota & North Dakota CRD 90 & 38090 & La Moure & 38045 & Northern-plains \\
\hline North Dakota & North Dakota CRD 90 & 38090 & Logan & 38047 & Northern-plains \\
\hline North Dakota & North Dakota CRD 20 & 38020 & McHenry & 38049 & Northern-plains \\
\hline North Dakota & North Dakota CRD 90 & 38090 & McIntosh & 38051 & Northern-plains \\
\hline North Dakota & North Dakota CRD 40 & 38040 & McKenzie & 38053 & Northern-plains \\
\hline North Dakota & North Dakota CRD 40 & 38040 & McLean & 38055 & Northern-plains \\
\hline North Dakota & North Dakota CRD 40 & 38040 & Mercer & 38057 & Northern-plains \\
\hline North Dakota & North Dakota CRD 80 & 38080 & Morton & 38059 & Northern-plains \\
\hline North Dakota & North Dakota CRD 10 & 38010 & Mountrail & 38061 & Northern-plains \\
\hline North Dakota & North Dakota CRD 30 & 38030 & Nelson & 38063 & Northern-plains \\
\hline North Dakota & North Dakota CRD 40 & 38040 & Oliver & 38065 & Northern-plains \\
\hline North Dakota & North Dakota CRD 30 & 38030 & Pembina & 38067 & Northern-plains \\
\hline North Dakota & North Dakota CRD 20 & 38020 & Pierce & 38069 & Northern-plains \\
\hline North Dakota & North Dakota CRD 30 & 38030 & Ramsey & 38071 & Northern-plains \\
\hline North Dakota & North Dakota CRD 90 & 38090 & Ransom & 38073 & Northern-plains \\
\hline North Dakota & North Dakota CRD 10 & 38010 & Renville & 38075 & Northern-plains \\
\hline North Dakota & North Dakota CRD 90 & 38090 & Richland & 38077 & Northern-plains \\
\hline North Dakota & North Dakota CRD 20 & 38020 & Rolette & 38079 & Northern-plains \\
\hline North Dakota & North Dakota CRD 90 & 38090 & Sargent & 38081 & Northern-plains \\
\hline North Dakota & North Dakota CRD 50 & 38050 & Sheridan & 38083 & Northern-plains \\
\hline North Dakota & North Dakota CRD 80 & 38080 & Sioux & 38085 & Northern-plains \\
\hline North Dakota & North Dakota CRD 70 & 38070 & Slope & 38087 & Northern-plains \\
\hline North Dakota & North Dakota CRD 70 & 38070 & Stark & 38089 & Northern-plains \\
\hline North Dakota & North Dakota CRD 60 & 38060 & Steele & 38091 & Northern-plains \\
\hline North Dakota & North Dakota CRD 50 & 38050 & Stutsman & 38093 & Northern-plains \\
\hline North Dakota & North Dakota CRD 30 & 38030 & Towner & 38095 & Northern-plains \\
\hline North Dakota & North Dakota CRD 60 & 38060 & Traill & 38097 & Northern-plains \\
\hline North Dakota & North Dakota CRD 30 & 38030 & Walsh & 38099 & Northern-plains \\
\hline North Dakota & North Dakota CRD 10 & 38010 & Ward & 38101 & Northern-plains \\
\hline North Dakota & North Dakota CRD 50 & 38050 & Wells & 38103 & Northern-plains \\
\hline North Dakota & North Dakota CRD 10 & 38010 & Williams & 38105 & Northern-plains \\
\hline Ohio & Ohio CRD 80 & 39080 & Adams & 39001 & Corn-belt \\
\hline Ohio & Ohio CRD 10 & 39010 & Allen & 39003 & Corn-belt \\
\hline Ohio & Ohio CRD 20 & 39020 & Ashland & 39005 & Corn-belt \\
\hline Ohio & Ohio CRD 30 & 39030 & Ashtabula & 39007 & Corn-belt \\
\hline Ohio & Ohio CRD 90 & 39090 & Athens & 39009 & Corn-belt \\
\hline Ohio & Ohio CRD 40 & 39040 & Auglaize & 39011 & Corn-belt \\
\hline Ohio & Ohio CRD 60 & 39060 & Belmont & 39013 & Corn-belt \\
\hline Ohio & Ohio CRD 80 & 39080 & Brown & 39015 & Corn-belt \\
\hline
\end{tabular}


Table 1. Crop Reporting Districts (CRD) of the conterminous United States and associated counties.-Continued [FIPS, Federal Information Processing Standard; USDA, U.S. Department of Agriculture]

\begin{tabular}{|c|c|c|c|c|c|}
\hline State & $\begin{array}{l}\text { Crop Reporting District } \\
\text { (CRD) }\end{array}$ & CRD Identifier & County Name & County FIPS & $\begin{array}{c}\text { USDA Farm Production } \\
\text { Region }\end{array}$ \\
\hline Ohio & Ohio CRD 70 & 39070 & Butler & 39017 & Corn-belt \\
\hline Ohio & Ohio CRD 60 & 39060 & Carroll & 39019 & Corn-belt \\
\hline Ohio & Ohio CRD 40 & 39040 & Champaign & 39021 & Corn-belt \\
\hline Ohio & Ohio CRD 40 & 39040 & Clark & 39023 & Corn-belt \\
\hline Ohio & Ohio CRD 70 & 39070 & Clermont & 39025 & Corn-belt \\
\hline Ohio & Ohio CRD 70 & 39070 & Clinton & 39027 & Corn-belt \\
\hline Ohio & Ohio CRD 30 & 39030 & Columbiana & 39029 & Corn-belt \\
\hline Ohio & Ohio CRD 60 & 39060 & Coshocton & 39031 & Corn-belt \\
\hline Ohio & Ohio CRD 20 & 39020 & Crawford & 39033 & Corn-belt \\
\hline Ohio & Ohio CRD 30 & 39030 & Cuyahoga & 39035 & Corn-belt \\
\hline Ohio & Ohio CRD 40 & 39040 & Darke & 39037 & Corn-belt \\
\hline Ohio & Ohio CRD 10 & 39010 & Defiance & 39039 & Corn-belt \\
\hline Ohio & Ohio CRD 50 & 39050 & Delaware & 39041 & Corn-belt \\
\hline Ohio & Ohio CRD 20 & 39020 & Erie & 39043 & Corn-belt \\
\hline Ohio & Ohio CRD 50 & 39050 & Fairfield & 39045 & Corn-belt \\
\hline Ohio & Ohio CRD 50 & 39050 & Fayette & 39047 & Corn-belt \\
\hline Ohio & Ohio CRD 50 & 39050 & Franklin & 39049 & Corn-belt \\
\hline Ohio & Ohio CRD 10 & 39010 & Fulton & 39051 & Corn-belt \\
\hline Ohio & Ohio CRD 80 & 39080 & Gallia & 39053 & Corn-belt \\
\hline Ohio & Ohio CRD 30 & 39030 & Geauga & 39055 & Corn-belt \\
\hline Ohio & Ohio CRD 70 & 39070 & Greene & 39057 & Corn-belt \\
\hline Ohio & Ohio CRD 90 & 39090 & Guernsey & 39059 & Corn-belt \\
\hline Ohio & Ohio CRD 70 & 39070 & Hamilton & 39061 & Corn-belt \\
\hline Ohio & Ohio CRD 10 & 39010 & Hancock & 39063 & Corn-belt \\
\hline Ohio & Ohio CRD 40 & 39040 & Hardin & 39065 & Corn-belt \\
\hline Ohio & Ohio CRD 60 & 39060 & Harrison & 39067 & Corn-belt \\
\hline Ohio & Ohio CRD 10 & 39010 & Henry & 39069 & Corn-belt \\
\hline Ohio & Ohio CRD 80 & 39080 & Highland & 39071 & Corn-belt \\
\hline Ohio & Ohio CRD 90 & 39090 & Hocking & 39073 & Corn-belt \\
\hline Ohio & Ohio CRD 60 & 39060 & Holmes & 39075 & Corn-belt \\
\hline Ohio & Ohio CRD 20 & 39020 & Huron & 39077 & Corn-belt \\
\hline Ohio & Ohio CRD 80 & 39080 & Jackson & 39079 & Corn-belt \\
\hline Ohio & Ohio CRD 60 & 39060 & Jefferson & 39081 & Corn-belt \\
\hline Ohio & Ohio CRD 50 & 39050 & Knox & 39083 & Corn-belt \\
\hline Ohio & Ohio CRD 30 & 39030 & Lake & 39085 & Corn-belt \\
\hline Ohio & Ohio CRD 80 & 39080 & Lawrence & 39087 & Corn-belt \\
\hline Ohio & Ohio CRD 50 & 39050 & Licking & 39089 & Corn-belt \\
\hline Ohio & Ohio CRD 40 & 39040 & Logan & 39091 & Corn-belt \\
\hline Ohio & Ohio CRD 20 & 39020 & Lorain & 39093 & Corn-belt \\
\hline Ohio & Ohio CRD 10 & 39010 & Lucas & 39095 & Corn-belt \\
\hline Ohio & Ohio CRD 50 & 39050 & Madison & 39097 & Corn-belt \\
\hline Ohio & Ohio CRD 30 & 39030 & Mahoning & 39099 & Corn-belt \\
\hline
\end{tabular}


Table 1. Crop Reporting Districts (CRD) of the conterminous United States and associated counties. - Continued

[FIPS, Federal Information Processing Standard; USDA, U.S. Department of Agriculture]

\begin{tabular}{|c|c|c|c|c|c|}
\hline State & $\begin{array}{l}\text { Crop Reporting District } \\
\text { (CRD) }\end{array}$ & CRD Identifier & County Name & County FIPS & $\begin{array}{c}\text { USDA Farm Production } \\
\text { Region }\end{array}$ \\
\hline Ohio & Ohio CRD 50 & 39050 & Marion & 39101 & Corn-belt \\
\hline Ohio & Ohio CRD 30 & 39030 & Medina & 39103 & Corn-belt \\
\hline Ohio & Ohio CRD 90 & 39090 & Meigs & 39105 & Corn-belt \\
\hline Ohio & Ohio CRD 40 & 39040 & Mercer & 39107 & Corn-belt \\
\hline Ohio & Ohio CRD 40 & 39040 & Miami & 39109 & Corn-belt \\
\hline Ohio & Ohio CRD 90 & 39090 & Monroe & 39111 & Corn-belt \\
\hline Ohio & Ohio CRD 70 & 39070 & Montgomery & 39113 & Corn-belt \\
\hline Ohio & Ohio CRD 90 & 39090 & Morgan & 39115 & Corn-belt \\
\hline Ohio & Ohio CRD 50 & 39050 & Morrow & 39117 & Corn-belt \\
\hline Ohio & Ohio CRD 90 & 39090 & Muskingum & 39119 & Corn-belt \\
\hline Ohio & Ohio CRD 90 & 39090 & Noble & 39121 & Corn-belt \\
\hline Ohio & Ohio CRD 20 & 39020 & Ottawa & 39123 & Corn-belt \\
\hline Ohio & Ohio CRD 10 & 39010 & Paulding & 39125 & Corn-belt \\
\hline Ohio & Ohio CRD 90 & 39090 & Perry & 39127 & Corn-belt \\
\hline Ohio & Ohio CRD 50 & 39050 & Pickaway & 39129 & Corn-belt \\
\hline Ohio & Ohio CRD 80 & 39080 & Pike & 39131 & Corn-belt \\
\hline Ohio & Ohio CRD 30 & 39030 & Portage & 39133 & Corn-belt \\
\hline Ohio & Ohio CRD 70 & 39070 & Preble & 39135 & Corn-belt \\
\hline Ohio & Ohio CRD 10 & 39010 & Putnam & 39137 & Corn-belt \\
\hline Ohio & Ohio CRD 20 & 39020 & Richland & 39139 & Corn-belt \\
\hline Ohio & Ohio CRD 50 & 39050 & Ross & 39141 & Corn-belt \\
\hline Ohio & Ohio CRD 20 & 39020 & Sandusky & 39143 & Corn-belt \\
\hline Ohio & Ohio CRD 80 & 39080 & Scioto & 39145 & Corn-belt \\
\hline Ohio & Ohio CRD 20 & 39020 & Seneca & 39147 & Corn-belt \\
\hline Ohio & Ohio CRD 40 & 39040 & Shelby & 39149 & Corn-belt \\
\hline Ohio & Ohio CRD 30 & 39030 & Stark & 39151 & Corn-belt \\
\hline Ohio & Ohio CRD 30 & 39030 & Summit & 39153 & Corn-belt \\
\hline Ohio & Ohio CRD 30 & 39030 & Trumbull & 39155 & Corn-belt \\
\hline Ohio & Ohio CRD 60 & 39060 & Tuscarawas & 39157 & Corn-belt \\
\hline Ohio & Ohio CRD 50 & 39050 & Union & 39159 & Corn-belt \\
\hline Ohio & Ohio CRD 10 & 39010 & Van Wert & 39161 & Corn-belt \\
\hline Ohio & Ohio CRD 90 & 39090 & Vinton & 39163 & Corn-belt \\
\hline Ohio & Ohio CRD 70 & 39070 & Warren & 39165 & Corn-belt \\
\hline Ohio & Ohio CRD 90 & 39090 & Washington & 39167 & Corn-belt \\
\hline Ohio & Ohio CRD 30 & 39030 & Wayne & 39169 & Corn-belt \\
\hline Ohio & Ohio CRD 10 & 39010 & Williams & 39171 & Corn-belt \\
\hline Ohio & Ohio CRD 10 & 39010 & Wood & 39173 & Corn-belt \\
\hline Ohio & Ohio CRD 20 & 39020 & Wyandot & 39175 & Corn-belt \\
\hline Oklahoma & Oklahoma CRD 80 & 40080 & Adair & 40001 & Southern-plains \\
\hline Oklahoma & Oklahoma CRD 40 & 40040 & Alfalfa & 40003 & Southern-plains \\
\hline Oklahoma & Oklahoma CRD 60 & 40060 & Atoka & 40005 & Southern-plains \\
\hline Oklahoma & Oklahoma CRD 10 & 40010 & Beaver & 40007 & Southern-plains \\
\hline
\end{tabular}


Table 1. Crop Reporting Districts (CRD) of the conterminous United States and associated counties.-Continued

[FIPS, Federal Information Processing Standard; USDA, U.S. Department of Agriculture]

\begin{tabular}{|c|c|c|c|c|c|}
\hline State & $\begin{array}{l}\text { Crop Reporting District } \\
\text { (CRD) }\end{array}$ & CRD Identifier & County Name & County FIPS & $\begin{array}{l}\text { USDA Farm Production } \\
\text { Region }\end{array}$ \\
\hline Oklahoma & Oklahoma CRD 20 & 40020 & Beckham & 40009 & Southern-plains \\
\hline Oklahoma & Oklahoma CRD 20 & 40020 & Blaine & 40011 & Southern-plains \\
\hline Oklahoma & Oklahoma CRD 60 & 40060 & Bryan & 40013 & Southern-plains \\
\hline Oklahoma & Oklahoma CRD 30 & 40030 & Caddo & 40015 & Southern-plains \\
\hline Oklahoma & Oklahoma CRD 50 & 40050 & Canadian & 40017 & Southern-plains \\
\hline Oklahoma & Oklahoma CRD 60 & 40060 & Carter & 40019 & Southern-plains \\
\hline Oklahoma & Oklahoma CRD 80 & 40080 & Cherokee & 40021 & Southern-plains \\
\hline Oklahoma & Oklahoma CRD 90 & 40090 & Choctaw & 40023 & Southern-plains \\
\hline Oklahoma & Oklahoma CRD 10 & 40010 & Cimarron & 40025 & Southern-plains \\
\hline Oklahoma & Oklahoma CRD 50 & 40050 & Cleveland & 40027 & Southern-plains \\
\hline Oklahoma & Oklahoma CRD 60 & 40060 & Coal & 40029 & Southern-plains \\
\hline Oklahoma & Oklahoma CRD 30 & 40030 & Comanche & 40031 & Southern-plains \\
\hline Oklahoma & Oklahoma CRD 30 & 40030 & Cotton & 40033 & Southern-plains \\
\hline Oklahoma & Oklahoma CRD 70 & 40070 & Craig & 40035 & Southern-plains \\
\hline Oklahoma & Oklahoma CRD 50 & 40050 & Creek & 40037 & Southern-plains \\
\hline Oklahoma & Oklahoma CRD 20 & 40020 & Custer & 40039 & Southern-plains \\
\hline Oklahoma & Oklahoma CRD 70 & 40070 & Delaware & 40041 & Southern-plains \\
\hline Oklahoma & Oklahoma CRD 20 & 40020 & Dewey & 40043 & Southern-plains \\
\hline Oklahoma & Oklahoma CRD 10 & 40010 & Ellis & 40045 & Southern-plains \\
\hline Oklahoma & Oklahoma CRD 40 & 40040 & Garfield & 40047 & Southern-plains \\
\hline Oklahoma & Oklahoma CRD 60 & 40060 & Garvin & 40049 & Southern-plains \\
\hline Oklahoma & Oklahoma CRD 50 & 40050 & Grady & 40051 & Southern-plains \\
\hline Oklahoma & Oklahoma CRD 40 & 40040 & Grant & 40053 & Southern-plains \\
\hline Oklahoma & Oklahoma CRD 30 & 40030 & Greer & 40055 & Southern-plains \\
\hline Oklahoma & Oklahoma CRD 30 & 40030 & Harmon & 40057 & Southern-plains \\
\hline Oklahoma & Oklahoma CRD 10 & 40010 & Harper & 40059 & Southern-plains \\
\hline Oklahoma & Oklahoma CRD 80 & 40080 & Haskell & 40061 & Southern-plains \\
\hline Oklahoma & Oklahoma CRD 80 & 40080 & Hughes & 40063 & Southern-plains \\
\hline Oklahoma & Oklahoma CRD 30 & 40030 & Jackson & 40065 & Southern-plains \\
\hline Oklahoma & Oklahoma CRD 60 & 40060 & Jefferson & 40067 & Southern-plains \\
\hline Oklahoma & Oklahoma CRD 60 & 40060 & Johnston & 40069 & Southern-plains \\
\hline Oklahoma & Oklahoma CRD 40 & 40040 & Kay & 40071 & Southern-plains \\
\hline Oklahoma & Oklahoma CRD 50 & 40050 & Kingfisher & 40073 & Southern-plains \\
\hline Oklahoma & Oklahoma CRD 30 & 40030 & Kiowa & 40075 & Southern-plains \\
\hline Oklahoma & Oklahoma CRD 90 & 40090 & Latimer & 40077 & Southern-plains \\
\hline Oklahoma & Oklahoma CRD 90 & 40090 & Le Flore & 40079 & Southern-plains \\
\hline Oklahoma & Oklahoma CRD 50 & 40050 & Lincoln & 40081 & Southern-plains \\
\hline Oklahoma & Oklahoma CRD 50 & 40050 & Logan & 40083 & Southern-plains \\
\hline Oklahoma & Oklahoma CRD 60 & 40060 & Love & 40085 & Southern-plains \\
\hline Oklahoma & Oklahoma CRD 50 & 40050 & McClain & 40087 & Southern-plains \\
\hline Oklahoma & Oklahoma CRD 90 & 40090 & McCurtain & 40089 & Southern-plains \\
\hline Oklahoma & Oklahoma CRD 80 & 40080 & McIntosh & 40091 & Southern-plains \\
\hline
\end{tabular}


Table 1. Crop Reporting Districts (CRD) of the conterminous United States and associated counties. - Continued

[FIPS, Federal Information Processing Standard; USDA, U.S. Department of Agriculture]

\begin{tabular}{|c|c|c|c|c|c|}
\hline State & $\begin{array}{l}\text { Crop Reporting District } \\
\text { (CRD) }\end{array}$ & CRD Identifier & County Name & County FIPS & $\begin{array}{c}\text { USDA Farm Production } \\
\text { Region }\end{array}$ \\
\hline Oklahoma & Oklahoma CRD 40 & 40040 & Major & 40093 & Southern-plains \\
\hline Oklahoma & Oklahoma CRD 60 & 40060 & Marshall & 40095 & Southern-plains \\
\hline Oklahoma & Oklahoma CRD 70 & 40070 & Mayes & 40097 & Southern-plains \\
\hline Oklahoma & Oklahoma CRD 60 & 40060 & Murray & 40099 & Southern-plains \\
\hline Oklahoma & Oklahoma CRD 80 & 40080 & Muskogee & 40101 & Southern-plains \\
\hline Oklahoma & Oklahoma CRD 40 & 40040 & Noble & 40103 & Southern-plains \\
\hline Oklahoma & Oklahoma CRD 70 & 40070 & Nowata & 40105 & Southern-plains \\
\hline Oklahoma & Oklahoma CRD 50 & 40050 & Okfuskee & 40107 & Southern-plains \\
\hline Oklahoma & Oklahoma CRD 50 & 40050 & Oklahoma & 40109 & Southern-plains \\
\hline Oklahoma & Oklahoma CRD 80 & 40080 & Okmulgee & 40111 & Southern-plains \\
\hline Oklahoma & Oklahoma CRD 70 & 40070 & Osage & 40113 & Southern-plains \\
\hline Oklahoma & Oklahoma CRD 70 & 40070 & Ottawa & 40115 & Southern-plains \\
\hline Oklahoma & Oklahoma CRD 70 & 40070 & Pawnee & 40117 & Southern-plains \\
\hline Oklahoma & Oklahoma CRD 50 & 40050 & Payne & 40119 & Southern-plains \\
\hline Oklahoma & Oklahoma CRD 80 & 40080 & Pittsburg & 40121 & Southern-plains \\
\hline Oklahoma & Oklahoma CRD 60 & 40060 & Pontotoc & 40123 & Southern-plains \\
\hline Oklahoma & Oklahoma CRD 50 & 40050 & Pottawatomie & 40125 & Southern-plains \\
\hline Oklahoma & Oklahoma CRD 90 & 40090 & Pushmataha & 40127 & Southern-plains \\
\hline Oklahoma & Oklahoma CRD 20 & 40020 & Roger Mills & 40129 & Southern-plains \\
\hline Oklahoma & Oklahoma CRD 70 & 40070 & Rogers & 40131 & Southern-plains \\
\hline Oklahoma & Oklahoma CRD 50 & 40050 & Seminole & 40133 & Southern-plains \\
\hline Oklahoma & Oklahoma CRD 80 & 40080 & Sequoyah & 40135 & Southern-plains \\
\hline Oklahoma & Oklahoma CRD 60 & 40060 & Stephens & 40137 & Southern-plains \\
\hline Oklahoma & Oklahoma CRD 10 & 40010 & Texas & 40139 & Southern-plains \\
\hline Oklahoma & Oklahoma CRD 30 & 40030 & Tillman & 40141 & Southern-plains \\
\hline Oklahoma & Oklahoma CRD 70 & 40070 & Tulsa & 40143 & Southern-plains \\
\hline Oklahoma & Oklahoma CRD 70 & 40070 & Wagoner & 40145 & Southern-plains \\
\hline Oklahoma & Oklahoma CRD 70 & 40070 & Washington & 40147 & Southern-plains \\
\hline Oklahoma & Oklahoma CRD 20 & 40020 & Washita & 40149 & Southern-plains \\
\hline Oklahoma & Oklahoma CRD 40 & 40040 & Woods & 40151 & Southern-plains \\
\hline Oklahoma & Oklahoma CRD 40 & 40040 & Woodward & 40153 & Southern-plains \\
\hline Oregon & Oregon CRD 30 & 41030 & Baker & 41001 & Pacific \\
\hline Oregon & Oregon CRD 10 & 41010 & Benton & 41003 & Pacific \\
\hline Oregon & Oregon CRD 10 & 41010 & Clackamas & 41005 & Pacific \\
\hline Oregon & Oregon CRD 10 & 41010 & Clatsop & 41007 & Pacific \\
\hline Oregon & Oregon CRD 10 & 41010 & Columbia & 41009 & Pacific \\
\hline Oregon & Oregon CRD 70 & 41070 & Coos & 41011 & Pacific \\
\hline Oregon & Oregon CRD 80 & 41080 & Crook & 41013 & Pacific \\
\hline Oregon & Oregon CRD 70 & 41070 & Curry & 41015 & Pacific \\
\hline Oregon & Oregon CRD 80 & 41080 & Deschutes & 41017 & Pacific \\
\hline Oregon & Oregon CRD 70 & 41070 & Douglas & 41019 & Pacific \\
\hline Oregon & Oregon CRD 20 & 41020 & Gilliam & 41021 & Pacific \\
\hline
\end{tabular}


Table 1. Crop Reporting Districts (CRD) of the conterminous United States and associated counties.-Continued

[FIPS, Federal Information Processing Standard; USDA, U.S. Department of Agriculture]

\begin{tabular}{|c|c|c|c|c|c|}
\hline State & $\begin{array}{l}\text { Crop Reporting District } \\
\text { (CRD) }\end{array}$ & CRD Identifier & County Name & County FIPS & $\begin{array}{c}\text { USDA Farm Production } \\
\text { Region }\end{array}$ \\
\hline Oregon & Oregon CRD 80 & 41080 & Grant & 41023 & Pacific \\
\hline Oregon & Oregon CRD 80 & 41080 & Harney & 41025 & Pacific \\
\hline Oregon & Oregon CRD 20 & 41020 & Hood River & 41027 & Pacific \\
\hline Oregon & Oregon CRD 70 & 41070 & Jackson & 41029 & Pacific \\
\hline Oregon & Oregon CRD 80 & 41080 & Jefferson & 41031 & Pacific \\
\hline Oregon & Oregon CRD 70 & 41070 & Josephine & 41033 & Pacific \\
\hline Oregon & Oregon CRD 80 & 41080 & Klamath & 41035 & Pacific \\
\hline Oregon & Oregon CRD 80 & 41080 & Lake & 41037 & Pacific \\
\hline Oregon & Oregon CRD 10 & 41010 & Lane & 41039 & Pacific \\
\hline Oregon & Oregon CRD 10 & 41010 & Lincoln & 41041 & Pacific \\
\hline Oregon & Oregon CRD 10 & 41010 & Linn & 41043 & Pacific \\
\hline Oregon & Oregon CRD 80 & 41080 & Malheur & 41045 & Pacific \\
\hline Oregon & Oregon CRD 10 & 41010 & Marion & 41047 & Pacific \\
\hline Oregon & Oregon CRD 20 & 41020 & Morrow & 41049 & Pacific \\
\hline Oregon & Oregon CRD 10 & 41010 & Multnomah & 41051 & Pacific \\
\hline Oregon & Oregon CRD 10 & 41010 & Polk & 41053 & Pacific \\
\hline Oregon & Oregon CRD 20 & 41020 & Sherman & 41055 & Pacific \\
\hline Oregon & Oregon CRD 10 & 41010 & Tillamook & 41057 & Pacific \\
\hline Oregon & Oregon CRD 30 & 41030 & Umatilla & 41059 & Pacific \\
\hline Oregon & Oregon CRD 30 & 41030 & Union & 41061 & Pacific \\
\hline Oregon & Oregon CRD 30 & 41030 & Wallowa & 41063 & Pacific \\
\hline Oregon & Oregon CRD 20 & 41020 & Wasco & 41065 & Pacific \\
\hline Oregon & Oregon CRD 10 & 41010 & Washington & 41067 & Pacific \\
\hline Oregon & Oregon CRD 80 & 41080 & Wheeler & 41069 & Pacific \\
\hline Oregon & Oregon CRD 10 & 41010 & Yamhill & 41071 & Pacific \\
\hline Pennsylvania & Pennsylvania CRD 80 & 42080 & Adams & 42001 & Northeast \\
\hline Pennsylvania & Pennsylvania CRD 70 & 42070 & Allegheny & 42003 & Northeast \\
\hline Pennsylvania & Pennsylvania CRD 40 & 42040 & Armstrong & 42005 & Northeast \\
\hline Pennsylvania & Pennsylvania CRD 40 & 42040 & Beaver & 42007 & Northeast \\
\hline Pennsylvania & Pennsylvania CRD 80 & 42080 & Bedford & 42009 & Northeast \\
\hline Pennsylvania & Pennsylvania CRD 90 & 42090 & Berks & 42011 & Northeast \\
\hline Pennsylvania & Pennsylvania CRD 50 & 42050 & Blair & 42013 & Northeast \\
\hline Pennsylvania & Pennsylvania CRD 20 & 42020 & Bradford & 42015 & Northeast \\
\hline Pennsylvania & Pennsylvania CRD 90 & 42090 & Bucks & 42017 & Northeast \\
\hline Pennsylvania & Pennsylvania CRD 40 & 42040 & Butler & 42019 & Northeast \\
\hline Pennsylvania & Pennsylvania CRD 50 & 42050 & Cambria & 42021 & Northeast \\
\hline Pennsylvania & Pennsylvania CRD 20 & 42020 & Cameron & 42023 & Northeast \\
\hline Pennsylvania & Pennsylvania CRD 60 & 42060 & Carbon & 42025 & Northeast \\
\hline Pennsylvania & Pennsylvania CRD 50 & 42050 & Centre & 42027 & Northeast \\
\hline Pennsylvania & Pennsylvania CRD 90 & 42090 & Chester & 42029 & Northeast \\
\hline Pennsylvania & Pennsylvania CRD 40 & 42040 & Clarion & 42031 & Northeast \\
\hline Pennsylvania & Pennsylvania CRD 50 & 42050 & Clearfield & 42033 & Northeast \\
\hline
\end{tabular}


Table 1. Crop Reporting Districts (CRD) of the conterminous United States and associated counties.-Continued

[FIPS, Federal Information Processing Standard; USDA, U.S. Department of Agriculture]

\begin{tabular}{|c|c|c|c|c|c|}
\hline State & $\begin{array}{l}\text { Crop Reporting District } \\
\text { (CRD) }\end{array}$ & CRD Identifier & County Name & County FIPS & $\begin{array}{c}\text { USDA Farm Production } \\
\text { Region }\end{array}$ \\
\hline Pennsylvania & Pennsylvania CRD 20 & 42020 & Clinton & 42035 & Northeast \\
\hline Pennsylvania & Pennsylvania CRD 50 & 42050 & Columbia & 42037 & Northeast \\
\hline Pennsylvania & Pennsylvania CRD 10 & 42010 & Crawford & 42039 & Northeast \\
\hline Pennsylvania & Pennsylvania CRD 80 & 42080 & Cumberland & 42041 & Northeast \\
\hline Pennsylvania & Pennsylvania CRD 50 & 42050 & Dauphin & 42043 & Northeast \\
\hline Pennsylvania & Pennsylvania CRD 90 & 42090 & Delaware & 42045 & Northeast \\
\hline Pennsylvania & Pennsylvania CRD 20 & 42020 & Elk & 42047 & Northeast \\
\hline Pennsylvania & Pennsylvania CRD 10 & 42010 & Erie & 42049 & Northeast \\
\hline Pennsylvania & Pennsylvania CRD 70 & 42070 & Fayette & 42051 & Northeast \\
\hline Pennsylvania & Pennsylvania CRD 10 & 42010 & Forest & 42053 & Northeast \\
\hline Pennsylvania & Pennsylvania CRD 80 & 42080 & Franklin & 42055 & Northeast \\
\hline Pennsylvania & Pennsylvania CRD 80 & 42080 & Fulton & 42057 & Northeast \\
\hline Pennsylvania & Pennsylvania CRD 70 & 42070 & Greene & 42059 & Northeast \\
\hline Pennsylvania & Pennsylvania CRD 50 & 42050 & Huntingdon & 42061 & Northeast \\
\hline Pennsylvania & Pennsylvania CRD 40 & 42040 & Indiana & 42063 & Northeast \\
\hline Pennsylvania & Pennsylvania CRD 40 & 42040 & Jefferson & 42065 & Northeast \\
\hline Pennsylvania & Pennsylvania CRD 50 & 42050 & Juniata & 42067 & Northeast \\
\hline Pennsylvania & Pennsylvania CRD 30 & 42030 & Lackawanna & 42069 & Northeast \\
\hline Pennsylvania & Pennsylvania CRD 90 & 42090 & Lancaster & 42071 & Northeast \\
\hline Pennsylvania & Pennsylvania CRD 40 & 42040 & Lawrence & 42073 & Northeast \\
\hline Pennsylvania & Pennsylvania CRD 90 & 42090 & Lebanon & 42075 & Northeast \\
\hline Pennsylvania & Pennsylvania CRD 60 & 42060 & Lehigh & 42077 & Northeast \\
\hline Pennsylvania & Pennsylvania CRD 60 & 42060 & Luzerne & 42079 & Northeast \\
\hline Pennsylvania & Pennsylvania CRD 20 & 42020 & Lycoming & 42081 & Northeast \\
\hline Pennsylvania & Pennsylvania CRD 20 & 42020 & Mckean & 42083 & Northeast \\
\hline Pennsylvania & Pennsylvania CRD 10 & 42010 & Mercer & 42085 & Northeast \\
\hline Pennsylvania & Pennsylvania CRD 50 & 42050 & Mifflin & 42087 & Northeast \\
\hline Pennsylvania & Pennsylvania CRD 60 & 42060 & Monroe & 42089 & Northeast \\
\hline Pennsylvania & Pennsylvania CRD 90 & 42090 & Montgomery & 42091 & Northeast \\
\hline Pennsylvania & Pennsylvania CRD 50 & 42050 & Montour & 42093 & Northeast \\
\hline Pennsylvania & Pennsylvania CRD 60 & 42060 & Northampton & 42095 & Northeast \\
\hline Pennsylvania & Pennsylvania CRD 50 & 42050 & Northumberland & 42097 & Northeast \\
\hline Pennsylvania & Pennsylvania CRD 50 & 42050 & Perry & 42099 & Northeast \\
\hline Pennsylvania & Pennsylvania CRD 90 & 42090 & Philadelphia & 42101 & Northeast \\
\hline Pennsylvania & Pennsylvania CRD 60 & 42060 & Pike & 42103 & Northeast \\
\hline Pennsylvania & Pennsylvania CRD 20 & 42020 & Potter & 42105 & Northeast \\
\hline Pennsylvania & Pennsylvania CRD 60 & 42060 & Schuylkill & 42107 & Northeast \\
\hline Pennsylvania & Pennsylvania CRD 50 & 42050 & Snyder & 42109 & Northeast \\
\hline Pennsylvania & Pennsylvania CRD 70 & 42070 & Somerset & 42111 & Northeast \\
\hline Pennsylvania & Pennsylvania CRD 20 & 42020 & Sullivan & 42113 & Northeast \\
\hline Pennsylvania & Pennsylvania CRD 30 & 42030 & Susquehanna & 42115 & Northeast \\
\hline Pennsylvania & Pennsylvania CRD 20 & 42020 & Tioga & 42117 & Northeast \\
\hline
\end{tabular}


Table 1. Crop Reporting Districts (CRD) of the conterminous United States and associated counties.-Continued

[FIPS, Federal Information Processing Standard; USDA, U.S. Department of Agriculture]

\begin{tabular}{|c|c|c|c|c|c|}
\hline State & $\begin{array}{l}\text { Crop Reporting District } \\
\text { (CRD) }\end{array}$ & CRD Identifier & County Name & County FIPS & $\begin{array}{c}\text { USDA Farm Production } \\
\text { Region }\end{array}$ \\
\hline Pennsylvania & Pennsylvania CRD 50 & 42050 & Union & 42119 & Northeast \\
\hline Pennsylvania & Pennsylvania CRD 10 & 42010 & Venango & 42121 & Northeast \\
\hline Pennsylvania & Pennsylvania CRD 10 & 42010 & Warren & 42123 & Northeast \\
\hline Pennsylvania & Pennsylvania CRD 70 & 42070 & Washington & 42125 & Northeast \\
\hline Pennsylvania & Pennsylvania CRD 30 & 42030 & Wayne & 42127 & Northeast \\
\hline Pennsylvania & Pennsylvania CRD 70 & 42070 & Westmoreland & 42129 & Northeast \\
\hline Pennsylvania & Pennsylvania CRD 30 & 42030 & Wyoming & 42131 & Northeast \\
\hline Pennsylvania & Pennsylvania CRD 80 & 42080 & York & 42133 & Northeast \\
\hline Rhode Island & Rhode island CRD 10 & 44010 & Bristol & 44001 & Northeast \\
\hline Rhode Island & Rhode island CRD 10 & 44010 & Kent & 44003 & Northeast \\
\hline Rhode Island & Rhode island CRD 10 & 44010 & Newport & 44005 & Northeast \\
\hline Rhode Island & Rhode island CRD 10 & 44010 & Providence & 44007 & Northeast \\
\hline Rhode Island & Rhode island CRD 10 & 44010 & Washington & 44009 & Northeast \\
\hline South Carolina & South Carolina CRD 40 & 45040 & Abbeville & 45001 & Southeast \\
\hline South Carolina & South Carolina CRD 40 & 45040 & Aiken & 45003 & Southeast \\
\hline South Carolina & South Carolina CRD 80 & 45080 & Allendale & 45005 & Southeast \\
\hline South Carolina & South Carolina CRD 10 & 45010 & Anderson & 45007 & Southeast \\
\hline South Carolina & South Carolina CRD 80 & 45080 & Bamberg & 45009 & Southeast \\
\hline South Carolina & South Carolina CRD 80 & 45080 & Barnwell & 45011 & Southeast \\
\hline South Carolina & South Carolina CRD 80 & 45080 & Beaufort & 45013 & Southeast \\
\hline South Carolina & South Carolina CRD 80 & 45080 & Berkeley & 45015 & Southeast \\
\hline South Carolina & South Carolina CRD 50 & 45050 & Calhoun & 45017 & Southeast \\
\hline South Carolina & South Carolina CRD 80 & 45080 & Charleston & 45019 & Southeast \\
\hline South Carolina & South Carolina CRD 10 & 45010 & Cherokee & 45021 & Southeast \\
\hline South Carolina & South Carolina CRD 20 & 45020 & Chester & 45023 & Southeast \\
\hline South Carolina & South Carolina CRD 30 & 45030 & Chesterfield & 45025 & Southeast \\
\hline South Carolina & South Carolina CRD 50 & 45050 & Clarendon & 45027 & Southeast \\
\hline South Carolina & South Carolina CRD 80 & 45080 & Colleton & 45029 & Southeast \\
\hline South Carolina & South Carolina CRD 30 & 45030 & Darlington & 45031 & Southeast \\
\hline South Carolina & South Carolina CRD 30 & 45030 & Dillon & 45033 & Southeast \\
\hline South Carolina & South Carolina CRD 80 & 45080 & Dorchester & 45035 & Southeast \\
\hline South Carolina & South Carolina CRD 40 & 45040 & Edgefield & 45037 & Southeast \\
\hline South Carolina & South Carolina CRD 20 & 45020 & Fairfield & 45039 & Southeast \\
\hline South Carolina & South Carolina CRD 30 & 45030 & Florence & 45041 & Southeast \\
\hline South Carolina & South Carolina CRD 30 & 45030 & Georgetown & 45043 & Southeast \\
\hline South Carolina & South Carolina CRD 10 & 45010 & Greenville & 45045 & Southeast \\
\hline South Carolina & South Carolina CRD 40 & 45040 & Greenwood & 45047 & Southeast \\
\hline South Carolina & South Carolina CRD 80 & 45080 & Hampton & 45049 & Southeast \\
\hline South Carolina & South Carolina CRD 30 & 45030 & Horry & 45051 & Southeast \\
\hline South Carolina & South Carolina CRD 80 & 45080 & Jasper & 45053 & Southeast \\
\hline South Carolina & South Carolina CRD 20 & 45020 & Kershaw & 45055 & Southeast \\
\hline South Carolina & South Carolina CRD 20 & 45020 & Lancaster & 45057 & Southeast \\
\hline
\end{tabular}


Table 1. Crop Reporting Districts (CRD) of the conterminous United States and associated counties.-Continued

[FIPS, Federal Information Processing Standard; USDA, U.S. Department of Agriculture]

\begin{tabular}{|c|c|c|c|c|c|}
\hline State & $\begin{array}{l}\text { Crop Reporting District } \\
\text { (CRD) }\end{array}$ & CRD Identifier & County Name & County FIPS & $\begin{array}{l}\text { USDA Farm Production } \\
\text { Region }\end{array}$ \\
\hline South Carolina & South Carolina CRD 10 & 45010 & Laurens & 45059 & Southeast \\
\hline South Carolina & South Carolina CRD 50 & 45050 & Lee & 45061 & Southeast \\
\hline South Carolina & South Carolina CRD 50 & 45050 & Lexington & 45063 & Southeast \\
\hline South Carolina & South Carolina CRD 40 & 45040 & McCormick & 45065 & Southeast \\
\hline South Carolina & South Carolina CRD 30 & 45030 & Marion & 45067 & Southeast \\
\hline South Carolina & South Carolina CRD 30 & 45030 & Marlboro & 45069 & Southeast \\
\hline South Carolina & South Carolina CRD 40 & 45040 & Newberry & 45071 & Southeast \\
\hline South Carolina & South Carolina CRD 10 & 45010 & Oconee & 45073 & Southeast \\
\hline South Carolina & South Carolina CRD 50 & 45050 & Orangeburg & 45075 & Southeast \\
\hline South Carolina & South Carolina CRD 10 & 45010 & Pickens & 45077 & Southeast \\
\hline South Carolina & South Carolina CRD 50 & 45050 & Richland & 45079 & Southeast \\
\hline South Carolina & South Carolina CRD 40 & 45040 & Saluda & 45081 & Southeast \\
\hline South Carolina & South Carolina CRD 10 & 45010 & Spartanburg & 45083 & Southeast \\
\hline South Carolina & South Carolina CRD 50 & 45050 & Sumter & 45085 & Southeast \\
\hline South Carolina & South Carolina CRD 10 & 45010 & Union & 45087 & Southeast \\
\hline South Carolina & South Carolina CRD 30 & 45030 & Williamsburg & 45089 & Southeast \\
\hline South Carolina & South Carolina CRD 20 & 45020 & York & 45091 & Southeast \\
\hline South Dakota & South Dakota CRD 50 & 46050 & Aurora & 46003 & Northern-plains \\
\hline South Dakota & South Dakota CRD 50 & 46050 & Beadle & 46005 & Northern-plains \\
\hline South Dakota & South Dakota CRD 70 & 46070 & Bennett & 46007 & Northern-plains \\
\hline South Dakota & South Dakota CRD 90 & 46090 & Bon Homme & 46009 & Northern-plains \\
\hline South Dakota & South Dakota CRD 60 & 46060 & Brookings & 46011 & Northern-plains \\
\hline South Dakota & South Dakota CRD 20 & 46020 & Brown & 46013 & Northern-plains \\
\hline South Dakota & South Dakota CRD 50 & 46050 & Brule & 46015 & Northern-plains \\
\hline South Dakota & South Dakota CRD 50 & 46050 & Buffalo & 46017 & Northern-plains \\
\hline South Dakota & South Dakota CRD 10 & 46010 & Butte & 46019 & Northern-plains \\
\hline South Dakota & South Dakota CRD 20 & 46020 & Campbell & 46021 & Northern-plains \\
\hline South Dakota & South Dakota CRD 90 & 46090 & Charles Mix & 46023 & Northern-plains \\
\hline South Dakota & South Dakota CRD 30 & 46030 & Clark & 46025 & Northern-plains \\
\hline South Dakota & South Dakota CRD 90 & 46090 & Clay & 46027 & Northern-plains \\
\hline South Dakota & South Dakota CRD 30 & 46030 & Codington & 46029 & Northern-plains \\
\hline South Dakota & South Dakota CRD 10 & 46010 & Corson & 46031 & Northern-plains \\
\hline South Dakota & South Dakota CRD 70 & 46070 & Custer & 46033 & Northern-plains \\
\hline South Dakota & South Dakota CRD 60 & 46060 & Davison & 46035 & Northern-plains \\
\hline South Dakota & South Dakota CRD 30 & 46030 & Day & 46037 & Northern-plains \\
\hline South Dakota & South Dakota CRD 30 & 46030 & Deuel & 46039 & Northern-plains \\
\hline South Dakota & South Dakota CRD 10 & 46010 & Dewey & 46041 & Northern-plains \\
\hline South Dakota & South Dakota CRD 90 & 46090 & Douglas & 46043 & Northern-plains \\
\hline South Dakota & South Dakota CRD 20 & 46020 & Edmunds & 46045 & Northern-plains \\
\hline South Dakota & South Dakota CRD 70 & 46070 & Fall River & 46047 & Northern-plains \\
\hline South Dakota & South Dakota CRD 20 & 46020 & Faulk & 46049 & Northern-plains \\
\hline South Dakota & South Dakota CRD 30 & 46030 & Grant & 46051 & Northern-plains \\
\hline
\end{tabular}


Table 1. Crop Reporting Districts (CRD) of the conterminous United States and associated counties.—Continued

[FIPS, Federal Information Processing Standard; USDA, U.S. Department of Agriculture]

\begin{tabular}{|c|c|c|c|c|c|}
\hline State & $\begin{array}{l}\text { Crop Reporting District } \\
\text { (CRD) }\end{array}$ & CRD Identifier & County Name & County FIPS & $\begin{array}{c}\text { USDA Farm Production } \\
\text { Region }\end{array}$ \\
\hline South Dakota & South Dakota CRD 80 & 46080 & Gregory & 46053 & Northern-plains \\
\hline South Dakota & South Dakota CRD 40 & 46040 & Haakon & 46055 & Northern-plains \\
\hline South Dakota & South Dakota CRD 30 & 46030 & Hamlin & 46057 & Northern-plains \\
\hline South Dakota & South Dakota CRD 50 & 46050 & Hand & 46059 & Northern-plains \\
\hline South Dakota & South Dakota CRD 60 & 46060 & Hanson & 46061 & Northern-plains \\
\hline South Dakota & South Dakota CRD 10 & 46010 & Harding & 46063 & Northern-plains \\
\hline South Dakota & South Dakota CRD 50 & 46050 & Hughes & 46065 & Northern-plains \\
\hline South Dakota & South Dakota CRD 90 & 46090 & Hutchinson & 46067 & Northern-plains \\
\hline South Dakota & South Dakota CRD 50 & 46050 & Hyde & 46069 & Northern-plains \\
\hline South Dakota & South Dakota CRD 40 & 46040 & Jackson & 46071 & Northern-plains \\
\hline South Dakota & South Dakota CRD 50 & 46050 & Jerauld & 46073 & Northern-plains \\
\hline South Dakota & South Dakota CRD 80 & 46080 & Jones & 46075 & Northern-plains \\
\hline South Dakota & South Dakota CRD 60 & 46060 & Kingsbury & 46077 & Northern-plains \\
\hline South Dakota & South Dakota CRD 60 & 46060 & Lake & 46079 & Northern-plains \\
\hline South Dakota & South Dakota CRD 40 & 46040 & Lawrence & 46081 & Northern-plains \\
\hline South Dakota & South Dakota CRD 90 & 46090 & Lincoln & 46083 & Northern-plains \\
\hline South Dakota & South Dakota CRD 80 & 46080 & Lyman & 46085 & Northern-plains \\
\hline South Dakota & South Dakota CRD 60 & 46060 & Mccook & 46087 & Northern-plains \\
\hline South Dakota & South Dakota CRD 20 & 46020 & McPherson & 46089 & Northern-plains \\
\hline South Dakota & South Dakota CRD 30 & 46030 & Marshall & 46091 & Northern-plains \\
\hline South Dakota & South Dakota CRD 40 & 46040 & Meade & 46093 & Northern-plains \\
\hline South Dakota & South Dakota CRD 80 & 46080 & Mellette & 46095 & Northern-plains \\
\hline South Dakota & South Dakota CRD 60 & 46060 & Miner & 46097 & Northern-plains \\
\hline South Dakota & South Dakota CRD 60 & 46060 & Minnehaha & 46099 & Northern-plains \\
\hline South Dakota & South Dakota CRD 60 & 46060 & Moody & 46101 & Northern-plains \\
\hline South Dakota & South Dakota CRD 40 & 46040 & Pennington & 46103 & Northern-plains \\
\hline South Dakota & South Dakota CRD 10 & 46010 & Perkins & 46105 & Northern-plains \\
\hline South Dakota & South Dakota CRD 20 & 46020 & Potter & 46107 & Northern-plains \\
\hline South Dakota & South Dakota CRD 30 & 46030 & Roberts & 46109 & Northern-plains \\
\hline South Dakota & South Dakota CRD 60 & 46060 & Sanborn & 46111 & Northern-plains \\
\hline South Dakota & South Dakota CRD 70 & 46070 & Shannon & 46113 & Northern-plains \\
\hline South Dakota & South Dakota CRD 20 & 46020 & Spink & 46115 & Northern-plains \\
\hline South Dakota & South Dakota CRD 40 & 46040 & Stanley & 46117 & Northern-plains \\
\hline South Dakota & South Dakota CRD 50 & 46050 & Sully & 46119 & Northern-plains \\
\hline South Dakota & South Dakota CRD 80 & 46080 & Todd & 46121 & Northern-plains \\
\hline South Dakota & South Dakota CRD 80 & 46080 & Tripp & 46123 & Northern-plains \\
\hline South Dakota & South Dakota CRD 90 & 46090 & Turner & 46125 & Northern-plains \\
\hline South Dakota & South Dakota CRD 90 & 46090 & Union & 46127 & Northern-plains \\
\hline South Dakota & South Dakota CRD 20 & 46020 & Walworth & 46129 & Northern-plains \\
\hline South Dakota & South Dakota CRD 90 & 46090 & Yankton & 46135 & Northern-plains \\
\hline South Dakota & South Dakota CRD 10 & 46010 & Ziebach & 46137 & Northern-plains \\
\hline Tennessee & Tennessee CRD 60 & 47060 & Anderson & 47001 & Appalachian \\
\hline
\end{tabular}


Table 1. Crop Reporting Districts (CRD) of the conterminous United States and associated counties. - Continued

[FIPS, Federal Information Processing Standard; USDA, U.S. Department of Agriculture]

\begin{tabular}{|c|c|c|c|c|c|}
\hline State & $\begin{array}{l}\text { Crop Reporting District } \\
\text { (CRD) }\end{array}$ & CRD Identifier & County Name & County FIPS & $\begin{array}{l}\text { USDA Farm Production } \\
\text { Region }\end{array}$ \\
\hline Tennessee & Tennessee CRD 40 & 47040 & Bedford & 47003 & Appalachian \\
\hline Tennessee & Tennessee CRD 20 & 47020 & Benton & 47005 & Appalachian \\
\hline Tennessee & Tennessee CRD 50 & 47050 & Bledsoe & 47007 & Appalachian \\
\hline Tennessee & Tennessee CRD 60 & 47060 & Blount & 47009 & Appalachian \\
\hline Tennessee & Tennessee CRD 60 & 47060 & Bradley & 47011 & Appalachian \\
\hline Tennessee & Tennessee CRD 60 & 47060 & Campbell & 47013 & Appalachian \\
\hline Tennessee & Tennessee CRD 40 & 47040 & Cannon & 47015 & Appalachian \\
\hline Tennessee & Tennessee CRD 20 & 47020 & Carroll & 47017 & Appalachian \\
\hline Tennessee & Tennessee CRD 60 & 47060 & Carter & 47019 & Appalachian \\
\hline Tennessee & Tennessee CRD 30 & 47030 & Cheatham & 47021 & Appalachian \\
\hline Tennessee & Tennessee CRD 20 & 47020 & Chester & 47023 & Appalachian \\
\hline Tennessee & Tennessee CRD 60 & 47060 & Claiborne & 47025 & Appalachian \\
\hline Tennessee & Tennessee CRD 40 & 47040 & Clay & 47027 & Appalachian \\
\hline Tennessee & Tennessee CRD 60 & 47060 & Cocke & 47029 & Appalachian \\
\hline Tennessee & Tennessee CRD 50 & 47050 & Coffee & 47031 & Appalachian \\
\hline Tennessee & Tennessee CRD 20 & 47020 & Crockett & 47033 & Appalachian \\
\hline Tennessee & Tennessee CRD 50 & 47050 & Cumberland & 47035 & Appalachian \\
\hline Tennessee & Tennessee CRD 40 & 47040 & Davidson & 47037 & Appalachian \\
\hline Tennessee & Tennessee CRD 20 & 47020 & Decatur & 47039 & Appalachian \\
\hline Tennessee & Tennessee CRD 40 & 47040 & De Kalb & 47041 & Appalachian \\
\hline Tennessee & Tennessee CRD 30 & 47030 & Dickson & 47043 & Appalachian \\
\hline Tennessee & Tennessee CRD 10 & 47010 & Dyer & 47045 & Appalachian \\
\hline Tennessee & Tennessee CRD 20 & 47020 & Fayette & 47047 & Appalachian \\
\hline Tennessee & Tennessee CRD 50 & 47050 & Fentress & 47049 & Appalachian \\
\hline Tennessee & Tennessee CRD 50 & 47050 & Franklin & 47051 & Appalachian \\
\hline Tennessee & Tennessee CRD 20 & 47020 & Gibson & 47053 & Appalachian \\
\hline Tennessee & Tennessee CRD 40 & 47040 & Giles & 47055 & Appalachian \\
\hline Tennessee & Tennessee CRD 60 & 47060 & Grainger & 47057 & Appalachian \\
\hline Tennessee & Tennessee CRD 60 & 47060 & Greene & 47059 & Appalachian \\
\hline Tennessee & Tennessee CRD 50 & 47050 & Grundy & 47061 & Appalachian \\
\hline Tennessee & Tennessee CRD 60 & 47060 & Hamblen & 47063 & Appalachian \\
\hline Tennessee & Tennessee CRD 60 & 47060 & Hamilton & 47065 & Appalachian \\
\hline Tennessee & Tennessee CRD 60 & 47060 & Hancock & 47067 & Appalachian \\
\hline Tennessee & Tennessee CRD 20 & 47020 & Hardeman & 47069 & Appalachian \\
\hline Tennessee & Tennessee CRD 20 & 47020 & Hardin & 47071 & Appalachian \\
\hline Tennessee & Tennessee CRD 60 & 47060 & Hawkins & 47073 & Appalachian \\
\hline Tennessee & Tennessee CRD 20 & 47020 & Haywood & 47075 & Appalachian \\
\hline Tennessee & Tennessee CRD 20 & 47020 & Henderson & 47077 & Appalachian \\
\hline Tennessee & Tennessee CRD 20 & 47020 & Henry & 47079 & Appalachian \\
\hline Tennessee & Tennessee CRD 30 & 47030 & Hickman & 47081 & Appalachian \\
\hline Tennessee & Tennessee CRD 30 & 47030 & Houston & 47083 & Appalachian \\
\hline Tennessee & Tennessee CRD 30 & 47030 & Humphreys & 47085 & Appalachian \\
\hline
\end{tabular}


Table 1. Crop Reporting Districts (CRD) of the conterminous United States and associated counties. - Continued

[FIPS, Federal Information Processing Standard; USDA, U.S. Department of Agriculture]

\begin{tabular}{|c|c|c|c|c|c|}
\hline State & $\begin{array}{l}\text { Crop Reporting District } \\
\text { (CRD) }\end{array}$ & CRD Identifier & County Name & County FIPS & $\begin{array}{c}\text { USDA Farm Production } \\
\text { Region }\end{array}$ \\
\hline Tennessee & Tennessee CRD 40 & 47040 & Jackson & 47087 & Appalachian \\
\hline Tennessee & Tennessee CRD 60 & 47060 & Jefferson & 47089 & Appalachian \\
\hline Tennessee & Tennessee CRD 60 & 47060 & Johnson & 47091 & Appalachian \\
\hline Tennessee & Tennessee CRD 60 & 47060 & Knox & 47093 & Appalachian \\
\hline Tennessee & Tennessee CRD 10 & 47010 & Lake & 47095 & Appalachian \\
\hline Tennessee & Tennessee CRD 10 & 47010 & Lauderdale & 47097 & Appalachian \\
\hline Tennessee & Tennessee CRD 30 & 47030 & Lawrence & 47099 & Appalachian \\
\hline Tennessee & Tennessee CRD 30 & 47030 & Lewis & 47101 & Appalachian \\
\hline Tennessee & Tennessee CRD 40 & 47040 & Lincoln & 47103 & Appalachian \\
\hline Tennessee & Tennessee CRD 60 & 47060 & Loudon & 47105 & Appalachian \\
\hline Tennessee & Tennessee CRD 60 & 47060 & Mcminn & 47107 & Appalachian \\
\hline Tennessee & Tennessee CRD 20 & 47020 & Mcnairy & 47109 & Appalachian \\
\hline Tennessee & Tennessee CRD 40 & 47040 & Macon & 47111 & Appalachian \\
\hline Tennessee & Tennessee CRD 20 & 47020 & Madison & 47113 & Appalachian \\
\hline Tennessee & Tennessee CRD 50 & 47050 & Marion & 47115 & Appalachian \\
\hline Tennessee & Tennessee CRD 40 & 47040 & Marshall & 47117 & Appalachian \\
\hline Tennessee & Tennessee CRD 40 & 47040 & Maury & 47119 & Appalachian \\
\hline Tennessee & Tennessee CRD 60 & 47060 & Meigs & 47121 & Appalachian \\
\hline Tennessee & Tennessee CRD 60 & 47060 & Monroe & 47123 & Appalachian \\
\hline Tennessee & Tennessee CRD 30 & 47030 & Montgomery & 47125 & Appalachian \\
\hline Tennessee & Tennessee CRD 40 & 47040 & Moore & 47127 & Appalachian \\
\hline Tennessee & Tennessee CRD 50 & 47050 & Morgan & 47129 & Appalachian \\
\hline Tennessee & Tennessee CRD 10 & 47010 & Obion & 47131 & Appalachian \\
\hline Tennessee & Tennessee CRD 50 & 47050 & Overton & 47133 & Appalachian \\
\hline Tennessee & Tennessee CRD 30 & 47030 & Perry & 47135 & Appalachian \\
\hline Tennessee & Tennessee CRD 50 & 47050 & Pickett & 47137 & Appalachian \\
\hline Tennessee & Tennessee CRD 60 & 47060 & Polk & 47139 & Appalachian \\
\hline Tennessee & Tennessee CRD 50 & 47050 & Putnam & 47141 & Appalachian \\
\hline Tennessee & Tennessee CRD 60 & 47060 & Rhea & 47143 & Appalachian \\
\hline Tennessee & Tennessee CRD 60 & 47060 & Roane & 47145 & Appalachian \\
\hline Tennessee & Tennessee CRD 30 & 47030 & Robertson & 47147 & Appalachian \\
\hline Tennessee & Tennessee CRD 40 & 47040 & Rutherford & 47149 & Appalachian \\
\hline Tennessee & Tennessee CRD 50 & 47050 & Scott & 47151 & Appalachian \\
\hline Tennessee & Tennessee CRD 50 & 47050 & Sequatchie & 47153 & Appalachian \\
\hline Tennessee & Tennessee CRD 60 & 47060 & Sevier & 47155 & Appalachian \\
\hline Tennessee & Tennessee CRD 10 & 47010 & Shelby & 47157 & Appalachian \\
\hline Tennessee & Tennessee CRD 40 & 47040 & Smith & 47159 & Appalachian \\
\hline Tennessee & Tennessee CRD 30 & 47030 & Stewart & 47161 & Appalachian \\
\hline Tennessee & Tennessee CRD 60 & 47060 & Sullivan & 47163 & Appalachian \\
\hline Tennessee & Tennessee CRD 40 & 47040 & Sumner & 47165 & Appalachian \\
\hline Tennessee & Tennessee CRD 10 & 47010 & Tipton & 47167 & Appalachian \\
\hline Tennessee & Tennessee CRD 40 & 47040 & Trousdale & 47169 & Appalachian \\
\hline
\end{tabular}


Table 1. Crop Reporting Districts (CRD) of the conterminous United States and associated counties.-Continued

[FIPS, Federal Information Processing Standard; USDA, U.S. Department of Agriculture]

\begin{tabular}{|c|c|c|c|c|c|}
\hline State & $\begin{array}{l}\text { Crop Reporting District } \\
\text { (CRD) }\end{array}$ & CRD Identifier & County Name & County FIPS & $\begin{array}{c}\text { USDA Farm Production } \\
\text { Region }\end{array}$ \\
\hline Tennessee & Tennessee CRD 60 & 47060 & Unicoi & 47171 & Appalachian \\
\hline Tennessee & Tennessee CRD 60 & 47060 & Union & 47173 & Appalachian \\
\hline Tennessee & Tennessee CRD 50 & 47050 & Van Buren & 47175 & Appalachian \\
\hline Tennessee & Tennessee CRD 50 & 47050 & Warren & 47177 & Appalachian \\
\hline Tennessee & Tennessee CRD 60 & 47060 & Washington & 47179 & Appalachian \\
\hline Tennessee & Tennessee CRD 30 & 47030 & Wayne & 47181 & Appalachian \\
\hline Tennessee & Tennessee CRD 20 & 47020 & Weakley & 47183 & Appalachian \\
\hline Tennessee & Tennessee CRD 50 & 47050 & White & 47185 & Appalachian \\
\hline Tennessee & Tennessee CRD 40 & 47040 & Williamson & 47187 & Appalachian \\
\hline Tennessee & Tennessee CRD 40 & 47040 & Wilson & 47189 & Appalachian \\
\hline Texas & Texas CRD 51 & 48051 & Anderson & 48001 & Southern-plains \\
\hline Texas & Texas CRD 12 & 48012 & Andrews & 48003 & Southern-plains \\
\hline Texas & Texas CRD 52 & 48052 & Angelina & 48005 & Southern-plains \\
\hline Texas & Texas CRD 82 & 48082 & Aransas & 48007 & Southern-plains \\
\hline Texas & Texas CRD 30 & 48030 & Archer & 48009 & Southern-plains \\
\hline Texas & Texas CRD 11 & 48011 & Armstrong & 48011 & Southern-plains \\
\hline Texas & Texas CRD 96 & 48096 & Atascosa & 48013 & Southern-plains \\
\hline Texas & Texas CRD 81 & 48081 & Austin & 48015 & Southern-plains \\
\hline Texas & Texas CRD 12 & 48012 & Bailey & 48017 & Southern-plains \\
\hline Texas & Texas CRD 70 & 48070 & Bandera & 48019 & Southern-plains \\
\hline Texas & Texas CRD 81 & 48081 & Bastrop & 48021 & Southern-plains \\
\hline Texas & Texas CRD 22 & 48022 & Baylor & 48023 & Southern-plains \\
\hline Texas & Texas CRD 81 & 48081 & Bee & 48025 & Southern-plains \\
\hline Texas & Texas CRD 40 & 48040 & Bell & 48027 & Southern-plains \\
\hline Texas & Texas CRD 81 & 48081 & Bexar & 48029 & Southern-plains \\
\hline Texas & Texas CRD 70 & 48070 & Blanco & 48031 & Southern-plains \\
\hline Texas & Texas CRD 21 & 48021 & Borden & 48033 & Southern-plains \\
\hline Texas & Texas CRD 40 & 48040 & Bosque & 48035 & Southern-plains \\
\hline Texas & Texas CRD 51 & 48051 & Bowie & 48037 & Southern-plains \\
\hline Texas & Texas CRD 90 & 48090 & Brazoria & 48039 & Southern-plains \\
\hline Texas & Texas CRD 52 & 48052 & Brazos & 48041 & Southern-plains \\
\hline Texas & Texas CRD 60 & 48060 & Brewster & 48043 & Southern-plains \\
\hline Texas & Texas CRD 11 & 48011 & Briscoe & 48045 & Southern-plains \\
\hline Texas & Texas CRD 96 & 48096 & Brooks & 48047 & Southern-plains \\
\hline Texas & Texas CRD 30 & 48030 & Brown & 48049 & Southern-plains \\
\hline Texas & Texas CRD 81 & 48081 & Burleson & 48051 & Southern-plains \\
\hline Texas & Texas CRD 70 & 48070 & Burnet & 48053 & Southern-plains \\
\hline Texas & Texas CRD 81 & 48081 & Caldwell & 48055 & Southern-plains \\
\hline Texas & Texas CRD 90 & 48090 & Calhoun & 48057 & Southern-plains \\
\hline Texas & Texas CRD 30 & 48030 & Callahan & 48059 & Southern-plains \\
\hline Texas & Texas CRD 97 & 48097 & Cameron & 48061 & Southern-plains \\
\hline Texas & Texas CRD 51 & 48051 & Camp & 48063 & Southern-plains \\
\hline
\end{tabular}


Table 1. Crop Reporting Districts (CRD) of the conterminous United States and associated counties.-Continued

[FIPS, Federal Information Processing Standard; USDA, U.S. Department of Agriculture]

\begin{tabular}{|c|c|c|c|c|c|}
\hline State & $\begin{array}{l}\text { Crop Reporting District } \\
\text { (CRD) }\end{array}$ & CRD Identifier & County Name & County FIPS & $\begin{array}{c}\text { USDA Farm Production } \\
\text { Region }\end{array}$ \\
\hline Texas & Texas CRD 11 & 48011 & Carson & 48065 & Southern-plains \\
\hline Texas & Texas CRD 51 & 48051 & Cass & 48067 & Southern-plains \\
\hline Texas & Texas CRD 11 & 48011 & Castro & 48069 & Southern-plains \\
\hline Texas & Texas CRD 90 & 48090 & Chambers & 48071 & Southern-plains \\
\hline Texas & Texas CRD 51 & 48051 & Cherokee & 48073 & Southern-plains \\
\hline Texas & Texas CRD 21 & 48021 & Childress & 48075 & Southern-plains \\
\hline Texas & Texas CRD 30 & 48030 & Clay & 48077 & Southern-plains \\
\hline Texas & Texas CRD 12 & 48012 & Cochran & 48079 & Southern-plains \\
\hline Texas & Texas CRD 70 & 48070 & Coke & 48081 & Southern-plains \\
\hline Texas & Texas CRD 22 & 48022 & Coleman & 48083 & Southern-plains \\
\hline Texas & Texas CRD 40 & 48040 & Collin & 48085 & Southern-plains \\
\hline Texas & Texas CRD 21 & 48021 & Collingsworth & 48087 & Southern-plains \\
\hline Texas & Texas CRD 81 & 48081 & Colorado & 48089 & Southern-plains \\
\hline Texas & Texas CRD 81 & 48081 & Comal & 48091 & Southern-plains \\
\hline Texas & Texas CRD 30 & 48030 & Comanche & 48093 & Southern-plains \\
\hline Texas & Texas CRD 70 & 48070 & Concho & 48095 & Southern-plains \\
\hline Texas & Texas CRD 40 & 48040 & Cooke & 48097 & Southern-plains \\
\hline Texas & Texas CRD 40 & 48040 & Coryell & 48099 & Southern-plains \\
\hline Texas & Texas CRD 21 & 48021 & Cottle & 48101 & Southern-plains \\
\hline Texas & Texas CRD 60 & 48060 & Crane & 48103 & Southern-plains \\
\hline Texas & Texas CRD 70 & 48070 & Crockett & 48105 & Southern-plains \\
\hline Texas & Texas CRD 12 & 48012 & Crosby & 48107 & Southern-plains \\
\hline Texas & Texas CRD 60 & 48060 & Culberson & 48109 & Southern-plains \\
\hline Texas & Texas CRD 11 & 48011 & Dallam & 48111 & Southern-plains \\
\hline Texas & Texas CRD 40 & 48040 & Dallas & 48113 & Southern-plains \\
\hline Texas & Texas CRD 12 & 48012 & Dawson & 48115 & Southern-plains \\
\hline Texas & Texas CRD 11 & 48011 & Deaf Smith & 48117 & Southern-plains \\
\hline Texas & Texas CRD 40 & 48040 & Delta & 48119 & Southern-plains \\
\hline Texas & Texas CRD 40 & 48040 & Denton & 48121 & Southern-plains \\
\hline Texas & Texas CRD 81 & 48081 & De Witt & 48123 & Southern-plains \\
\hline Texas & Texas CRD 21 & 48021 & Dickens & 48125 & Southern-plains \\
\hline Texas & Texas CRD 96 & 48096 & Dimmit & 48127 & Southern-plains \\
\hline Texas & Texas CRD 21 & 48021 & Donley & 48129 & Southern-plains \\
\hline Texas & Texas CRD 96 & 48096 & Duval & 48131 & Southern-plains \\
\hline Texas & Texas CRD 30 & 48030 & Eastland & 48133 & Southern-plains \\
\hline Texas & Texas CRD 60 & 48060 & Ector & 48135 & Southern-plains \\
\hline Texas & Texas CRD 70 & 48070 & Edwards & 48137 & Southern-plains \\
\hline Texas & Texas CRD 40 & 48040 & Ellis & 48139 & Southern-plains \\
\hline Texas & Texas CRD 60 & 48060 & El Paso & 48141 & Southern-plains \\
\hline Texas & Texas CRD 30 & 48030 & Erath & 48143 & Southern-plains \\
\hline Texas & Texas CRD 40 & 48040 & Falls & 48145 & Southern-plains \\
\hline Texas & Texas CRD 40 & 48040 & Fannin & 48147 & Southern-plains \\
\hline
\end{tabular}


Table 1. Crop Reporting Districts (CRD) of the conterminous United States and associated counties. - Continued

[FIPS, Federal Information Processing Standard; USDA, U.S. Department of Agriculture]

\begin{tabular}{|c|c|c|c|c|c|}
\hline State & $\begin{array}{l}\text { Crop Reporting District } \\
\text { (CRD) }\end{array}$ & CRD Identifier & County Name & County FIPS & $\begin{array}{c}\text { USDA Farm Production } \\
\text { Region }\end{array}$ \\
\hline Texas & Texas CRD 81 & 48081 & Fayette & 48149 & Southern-plains \\
\hline Texas & Texas CRD 22 & 48022 & Fisher & 48151 & Southern-plains \\
\hline Texas & Texas CRD 11 & 48011 & Floyd & 48153 & Southern-plains \\
\hline Texas & Texas CRD 21 & 48021 & Foard & 48155 & Southern-plains \\
\hline Texas & Texas CRD 90 & 48090 & Fort Bend & 48157 & Southern-plains \\
\hline Texas & Texas CRD 51 & 48051 & Franklin & 48159 & Southern-plains \\
\hline Texas & Texas CRD 52 & 48052 & Freestone & 48161 & Southern-plains \\
\hline Texas & Texas CRD 96 & 48096 & Frio & 48163 & Southern-plains \\
\hline Texas & Texas CRD 12 & 48012 & Gaines & 48165 & Southern-plains \\
\hline Texas & Texas CRD 90 & 48090 & Galveston & 48167 & Southern-plains \\
\hline Texas & Texas CRD 21 & 48021 & Garza & 48169 & Southern-plains \\
\hline Texas & Texas CRD 70 & 48070 & Gillespie & 48171 & Southern-plains \\
\hline Texas & Texas CRD 12 & 48012 & Glasscock & 48173 & Southern-plains \\
\hline Texas & Texas CRD 81 & 48081 & Goliad & 48175 & Southern-plains \\
\hline Texas & Texas CRD 81 & 48081 & Gonzales & 48177 & Southern-plains \\
\hline Texas & Texas CRD 11 & 48011 & Gray & 48179 & Southern-plains \\
\hline Texas & Texas CRD 40 & 48040 & Grayson & 48181 & Southern-plains \\
\hline Texas & Texas CRD 51 & 48051 & Gregg & 48183 & Southern-plains \\
\hline Texas & Texas CRD 52 & 48052 & Grimes & 48185 & Southern-plains \\
\hline Texas & Texas CRD 81 & 48081 & Guadalupe & 48187 & Southern-plains \\
\hline Texas & Texas CRD 11 & 48011 & Hale & 48189 & Southern-plains \\
\hline Texas & Texas CRD 21 & 48021 & Hall & 48191 & Southern-plains \\
\hline Texas & Texas CRD 40 & 48040 & Hamilton & 48193 & Southern-plains \\
\hline Texas & Texas CRD 11 & 48011 & Hansford & 48195 & Southern-plains \\
\hline Texas & Texas CRD 21 & 48021 & Hardeman & 48197 & Southern-plains \\
\hline Texas & Texas CRD 52 & 48052 & Hardin & 48199 & Southern-plains \\
\hline Texas & Texas CRD 90 & 48090 & Harris & 48201 & Southern-plains \\
\hline Texas & Texas CRD 51 & 48051 & Harrison & 48203 & Southern-plains \\
\hline Texas & Texas CRD 11 & 48011 & Hartley & 48205 & Southern-plains \\
\hline Texas & Texas CRD 22 & 48022 & Haskell & 48207 & Southern-plains \\
\hline Texas & Texas CRD 81 & 48081 & Hays & 48209 & Southern-plains \\
\hline Texas & Texas CRD 11 & 48011 & Hemphill & 48211 & Southern-plains \\
\hline Texas & Texas CRD 51 & 48051 & Henderson & 48213 & Southern-plains \\
\hline Texas & Texas CRD 97 & 48097 & Hidalgo & 48215 & Southern-plains \\
\hline Texas & Texas CRD 40 & 48040 & Hill & 48217 & Southern-plains \\
\hline Texas & Texas CRD 12 & 48012 & Hockley & 48219 & Southern-plains \\
\hline Texas & Texas CRD 30 & 48030 & Hood & 48221 & Southern-plains \\
\hline Texas & Texas CRD 51 & 48051 & Hopkins & 48223 & Southern-plains \\
\hline Texas & Texas CRD 51 & 48051 & Houston & 48225 & Southern-plains \\
\hline Texas & Texas CRD 12 & 48012 & Howard & 48227 & Southern-plains \\
\hline Texas & Texas CRD 60 & 48060 & Hudspeth & 48229 & Southern-plains \\
\hline Texas & Texas CRD 40 & 48040 & Hunt & 48231 & Southern-plains \\
\hline
\end{tabular}


Table 1. Crop Reporting Districts (CRD) of the conterminous United States and associated counties.-Continued

[FIPS, Federal Information Processing Standard; USDA, U.S. Department of Agriculture]

\begin{tabular}{|c|c|c|c|c|c|}
\hline State & $\begin{array}{l}\text { Crop Reporting District } \\
\text { (CRD) }\end{array}$ & CRD Identifier & County Name & County FIPS & $\begin{array}{c}\text { USDA Farm Production } \\
\text { Region }\end{array}$ \\
\hline Texas & Texas CRD 11 & 48011 & Hutchinson & 48233 & Southern-plains \\
\hline Texas & Texas CRD 70 & 48070 & Irion & 48235 & Southern-plains \\
\hline Texas & Texas CRD 30 & 48030 & Jack & 48237 & Southern-plains \\
\hline Texas & Texas CRD 90 & 48090 & Jackson & 48239 & Southern-plains \\
\hline Texas & Texas CRD 52 & 48052 & Jasper & 48241 & Southern-plains \\
\hline Texas & Texas CRD 60 & 48060 & Jeff Davis & 48243 & Southern-plains \\
\hline Texas & Texas CRD 90 & 48090 & Jefferson & 48245 & Southern-plains \\
\hline Texas & Texas CRD 96 & 48096 & Jim Hogg & 48247 & Southern-plains \\
\hline Texas & Texas CRD 96 & 48096 & Jim Wells & 48249 & Southern-plains \\
\hline Texas & Texas CRD 40 & 48040 & Johnson & 48251 & Southern-plains \\
\hline Texas & Texas CRD 22 & 48022 & Jones & 48253 & Southern-plains \\
\hline Texas & Texas CRD 81 & 48081 & Karnes & 48255 & Southern-plains \\
\hline Texas & Texas CRD 40 & 48040 & Kaufman & 48257 & Southern-plains \\
\hline Texas & Texas CRD 70 & 48070 & Kendall & 48259 & Southern-plains \\
\hline Texas & Texas CRD 96 & 48096 & Kenedy & 48261 & Southern-plains \\
\hline Texas & Texas CRD 21 & 48021 & Kent & 48263 & Southern-plains \\
\hline Texas & Texas CRD 70 & 48070 & Kerr & 48265 & Southern-plains \\
\hline Texas & Texas CRD 70 & 48070 & Kimble & 48267 & Southern-plains \\
\hline Texas & Texas CRD 21 & 48021 & King & 48269 & Southern-plains \\
\hline Texas & Texas CRD 70 & 48070 & Kinney & 48271 & Southern-plains \\
\hline Texas & Texas CRD 82 & 48082 & Kleberg & 48273 & Southern-plains \\
\hline Texas & Texas CRD 22 & 48022 & Knox & 48275 & Southern-plains \\
\hline Texas & Texas CRD 40 & 48040 & Lamar & 48277 & Southern-plains \\
\hline Texas & Texas CRD 12 & 48012 & Lamb & 48279 & Southern-plains \\
\hline Texas & Texas CRD 70 & 48070 & Lampasas & 48281 & Southern-plains \\
\hline Texas & Texas CRD 96 & 48096 & LaSalle & 48283 & Southern-plains \\
\hline Texas & Texas CRD 81 & 48081 & Lavaca & 48285 & Southern-plains \\
\hline Texas & Texas CRD 81 & 48081 & Lee & 48287 & Southern-plains \\
\hline Texas & Texas CRD 52 & 48052 & Leon & 48289 & Southern-plains \\
\hline Texas & Texas CRD 90 & 48090 & Liberty & 48291 & Southern-plains \\
\hline Texas & Texas CRD 40 & 48040 & Limestone & 48293 & Southern-plains \\
\hline Texas & Texas CRD 11 & 48011 & Lipscomb & 48295 & Southern-plains \\
\hline Texas & Texas CRD 96 & 48096 & Live Oak & 48297 & Southern-plains \\
\hline Texas & Texas CRD 70 & 48070 & Llano & 48299 & Southern-plains \\
\hline Texas & Texas CRD 60 & 48060 & Loving & 48301 & Southern-plains \\
\hline Texas & Texas CRD 12 & 48012 & Lubbock & 48303 & Southern-plains \\
\hline Texas & Texas CRD 12 & 48012 & Lynn & 48305 & Southern-plains \\
\hline Texas & Texas CRD 70 & 48070 & McCulloch & 48307 & Southern-plains \\
\hline Texas & Texas CRD 40 & 48040 & McLennan & 48309 & Southern-plains \\
\hline Texas & Texas CRD 96 & 48096 & McMullen & 48311 & Southern-plains \\
\hline Texas & Texas CRD 52 & 48052 & Madison & 48313 & Southern-plains \\
\hline Texas & Texas CRD 51 & 48051 & Marion & 48315 & Southern-plains \\
\hline
\end{tabular}


Table 1. Crop Reporting Districts (CRD) of the conterminous United States and associated counties.-Continued

[FIPS, Federal Information Processing Standard; USDA, U.S. Department of Agriculture]

\begin{tabular}{|c|c|c|c|c|c|}
\hline State & $\begin{array}{l}\text { Crop Reporting District } \\
\text { (CRD) }\end{array}$ & CRD Identifier & County Name & County FIPS & $\begin{array}{c}\text { USDA Farm Production } \\
\text { Region }\end{array}$ \\
\hline Texas & Texas CRD 12 & 48012 & Martin & 48317 & Southern-plains \\
\hline Texas & Texas CRD 70 & 48070 & Mason & 48319 & Southern-plains \\
\hline Texas & Texas CRD 90 & 48090 & Matagorda & 48321 & Southern-plains \\
\hline Texas & Texas CRD 96 & 48096 & Maverick & 48323 & Southern-plains \\
\hline Texas & Texas CRD 81 & 48081 & Medina & 48325 & Southern-plains \\
\hline Texas & Texas CRD 70 & 48070 & Menard & 48327 & Southern-plains \\
\hline Texas & Texas CRD 12 & 48012 & Midland & 48329 & Southern-plains \\
\hline Texas & Texas CRD 40 & 48040 & Milam & 48331 & Southern-plains \\
\hline Texas & Texas CRD 30 & 48030 & Mills & 48333 & Southern-plains \\
\hline Texas & Texas CRD 22 & 48022 & Mitchell & 48335 & Southern-plains \\
\hline Texas & Texas CRD 30 & 48030 & Montague & 48337 & Southern-plains \\
\hline Texas & Texas CRD 52 & 48052 & Montgomery & 48339 & Southern-plains \\
\hline Texas & Texas CRD 11 & 48011 & Moore & 48341 & Southern-plains \\
\hline Texas & Texas CRD 51 & 48051 & Morris & 48343 & Southern-plains \\
\hline Texas & Texas CRD 21 & 48021 & Motley & 48345 & Southern-plains \\
\hline Texas & Texas CRD 51 & 48051 & Nacogdoches & 48347 & Southern-plains \\
\hline Texas & Texas CRD 40 & 48040 & Navarro & 48349 & Southern-plains \\
\hline Texas & Texas CRD 52 & 48052 & Newton & 48351 & Southern-plains \\
\hline Texas & Texas CRD 22 & 48022 & Nolan & 48353 & Southern-plains \\
\hline Texas & Texas CRD 82 & 48082 & Nueces & 48355 & Southern-plains \\
\hline Texas & Texas CRD 11 & 48011 & Ochiltree & 48357 & Southern-plains \\
\hline Texas & Texas CRD 11 & 48011 & Oldham & 48359 & Southern-plains \\
\hline Texas & Texas CRD 90 & 48090 & Orange & 48361 & Southern-plains \\
\hline Texas & Texas CRD 30 & 48030 & Palo Pinto & 48363 & Southern-plains \\
\hline Texas & Texas CRD 51 & 48051 & Panola & 48365 & Southern-plains \\
\hline Texas & Texas CRD 30 & 48030 & Parker & 48367 & Southern-plains \\
\hline Texas & Texas CRD 11 & 48011 & Parmer & 48369 & Southern-plains \\
\hline Texas & Texas CRD 60 & 48060 & Pecos & 48371 & Southern-plains \\
\hline Texas & Texas CRD 52 & 48052 & Polk & 48373 & Southern-plains \\
\hline Texas & Texas CRD 11 & 48011 & Potter & 48375 & Southern-plains \\
\hline Texas & Texas CRD 60 & 48060 & Presidio & 48377 & Southern-plains \\
\hline Texas & Texas CRD 51 & 48051 & Rains & 48379 & Southern-plains \\
\hline Texas & Texas CRD 11 & 48011 & Randall & 48381 & Southern-plains \\
\hline Texas & Texas CRD 70 & 48070 & Reagan & 48383 & Southern-plains \\
\hline Texas & Texas CRD 70 & 48070 & Real & 48385 & Southern-plains \\
\hline Texas & Texas CRD 51 & 48051 & Red River & 48387 & Southern-plains \\
\hline Texas & Texas CRD 60 & 48060 & Reeves & 48389 & Southern-plains \\
\hline Texas & Texas CRD 82 & 48082 & Refugio & 48391 & Southern-plains \\
\hline Texas & Texas CRD 11 & 48011 & Roberts & 48393 & Southern-plains \\
\hline Texas & Texas CRD 52 & 48052 & Robertson & 48395 & Southern-plains \\
\hline Texas & Texas CRD 40 & 48040 & Rockwall & 48397 & Southern-plains \\
\hline Texas & Texas CRD 22 & 48022 & Runnels & 48399 & Southern-plains \\
\hline
\end{tabular}


94 Method for Estimating Annual Atrazine Use for Counties in the Conterminous United States, 1992-2007

Table 1. Crop Reporting Districts (CRD) of the conterminous United States and associated counties.-Continued

[FIPS, Federal Information Processing Standard; USDA, U.S. Department of Agriculture]

\begin{tabular}{|c|c|c|c|c|c|}
\hline State & $\begin{array}{l}\text { Crop Reporting District } \\
\text { (CRD) }\end{array}$ & CRD Identifier & County Name & County FIPS & $\begin{array}{c}\text { USDA Farm Production } \\
\text { Region }\end{array}$ \\
\hline Texas & Texas CRD 51 & 48051 & Rusk & 48401 & Southern-plains \\
\hline Texas & Texas CRD 52 & 48052 & Sabine & 48403 & Southern-plains \\
\hline Texas & Texas CRD 52 & 48052 & San Augustine & 48405 & Southern-plains \\
\hline Texas & Texas CRD 52 & 48052 & San Jacinto & 48407 & Southern-plains \\
\hline Texas & Texas CRD 82 & 48082 & San Patricio & 48409 & Southern-plains \\
\hline Texas & Texas CRD 70 & 48070 & San Saba & 48411 & Southern-plains \\
\hline Texas & Texas CRD 70 & 48070 & Schleicher & 48413 & Southern-plains \\
\hline Texas & Texas CRD 22 & 48022 & Scurry & 48415 & Southern-plains \\
\hline Texas & Texas CRD 30 & 48030 & Shackelford & 48417 & Southern-plains \\
\hline Texas & Texas CRD 51 & 48051 & Shelby & 48419 & Southern-plains \\
\hline Texas & Texas CRD 11 & 48011 & Sherman & 48421 & Southern-plains \\
\hline Texas & Texas CRD 51 & 48051 & Smith & 48423 & Southern-plains \\
\hline Texas & Texas CRD 30 & 48030 & Somervell & 48425 & Southern-plains \\
\hline Texas & Texas CRD 97 & 48097 & Starr & 48427 & Southern-plains \\
\hline Texas & Texas CRD 30 & 48030 & Stephens & 48429 & Southern-plains \\
\hline Texas & Texas CRD 70 & 48070 & Sterling & 48431 & Southern-plains \\
\hline Texas & Texas CRD 22 & 48022 & Stonewall & 48433 & Southern-plains \\
\hline Texas & Texas CRD 70 & 48070 & Sutton & 48435 & Southern-plains \\
\hline Texas & Texas CRD 11 & 48011 & Swisher & 48437 & Southern-plains \\
\hline Texas & Texas CRD 40 & 48040 & Tarrant & 48439 & Southern-plains \\
\hline Texas & Texas CRD 22 & 48022 & Taylor & 48441 & Southern-plains \\
\hline Texas & Texas CRD 60 & 48060 & Terrell & 48443 & Southern-plains \\
\hline Texas & Texas CRD 12 & 48012 & Terry & 48445 & Southern-plains \\
\hline Texas & Texas CRD 30 & 48030 & Throckmorton & 48447 & Southern-plains \\
\hline Texas & Texas CRD 51 & 48051 & Titus & 48449 & Southern-plains \\
\hline Texas & Texas CRD 70 & 48070 & Tom Green & 48451 & Southern-plains \\
\hline Texas & Texas CRD 81 & 48081 & Travis & 48453 & Southern-plains \\
\hline Texas & Texas CRD 52 & 48052 & Trinity & 48455 & Southern-plains \\
\hline Texas & Texas CRD 52 & 48052 & Tyler & 48457 & Southern-plains \\
\hline Texas & Texas CRD 51 & 48051 & Upshur & 48459 & Southern-plains \\
\hline Texas & Texas CRD 70 & 48070 & Upton & 48461 & Southern-plains \\
\hline Texas & Texas CRD 70 & 48070 & Uvalde & 48463 & Southern-plains \\
\hline Texas & Texas CRD 70 & 48070 & Val Verde & 48465 & Southern-plains \\
\hline Texas & Texas CRD 51 & 48051 & Van Zandt & 48467 & Southern-plains \\
\hline Texas & Texas CRD 90 & 48090 & Victoria & 48469 & Southern-plains \\
\hline Texas & Texas CRD 52 & 48052 & Walker & 48471 & Southern-plains \\
\hline Texas & Texas CRD 52 & 48052 & Waller & 48473 & Southern-plains \\
\hline Texas & Texas CRD 60 & 48060 & Ward & 48475 & Southern-plains \\
\hline Texas & Texas CRD 81 & 48081 & Washington & 48477 & Southern-plains \\
\hline Texas & Texas CRD 96 & 48096 & Webb & 48479 & Southern-plains \\
\hline Texas & Texas CRD 90 & 48090 & Wharton & 48481 & Southern-plains \\
\hline Texas & Texas CRD 21 & 48021 & Wheeler & 48483 & Southern-plains \\
\hline
\end{tabular}


Table 1. Crop Reporting Districts (CRD) of the conterminous United States and associated counties.-Continued

[FIPS, Federal Information Processing Standard; USDA, U.S. Department of Agriculture]

\begin{tabular}{|c|c|c|c|c|c|}
\hline State & $\begin{array}{l}\text { Crop Reporting District } \\
\text { (CRD) }\end{array}$ & CRD Identifier & County Name & County FIPS & $\begin{array}{c}\text { USDA Farm Production } \\
\text { Region }\end{array}$ \\
\hline Texas & Texas CRD 21 & 48021 & Wichita & 48485 & Southern-plains \\
\hline Texas & Texas CRD 21 & 48021 & Wilbarger & 48487 & Southern-plains \\
\hline Texas & Texas CRD 97 & 48097 & Willacy & 48489 & Southern-plains \\
\hline Texas & Texas CRD 40 & 48040 & Williamson & 48491 & Southern-plains \\
\hline Texas & Texas CRD 81 & 48081 & Wilson & 48493 & Southern-plains \\
\hline Texas & Texas CRD 60 & 48060 & Winkler & 48495 & Southern-plains \\
\hline Texas & Texas CRD 30 & 48030 & Wise & 48497 & Southern-plains \\
\hline Texas & Texas CRD 51 & 48051 & Wood & 48499 & Southern-plains \\
\hline Texas & Texas CRD 12 & 48012 & Yoakum & 48501 & Southern-plains \\
\hline Texas & Texas CRD 30 & 48030 & Young & 48503 & Southern-plains \\
\hline Texas & Texas CRD 96 & 48096 & Zapata & 48505 & Southern-plains \\
\hline Texas & Texas CRD 96 & 48096 & Zavala & 48507 & Southern-plains \\
\hline Utah & Utah CRD 70 & 49070 & Beaver & 49001 & Mountain \\
\hline Utah & Utah CRD 10 & 49010 & Box Elder & 49003 & Mountain \\
\hline Utah & Utah CRD 10 & 49010 & Cache & 49005 & Mountain \\
\hline Utah & Utah CRD 60 & 49060 & Carbon & 49007 & Mountain \\
\hline Utah & Utah CRD 60 & 49060 & Daggett & 49009 & Mountain \\
\hline Utah & Utah CRD 10 & 49010 & Davis & 49011 & Mountain \\
\hline Utah & Utah CRD 60 & 49060 & Duchesne & 49013 & Mountain \\
\hline Utah & Utah CRD 60 & 49060 & Emery & 49015 & Mountain \\
\hline Utah & Utah CRD 70 & 49070 & Garfield & 49017 & Mountain \\
\hline Utah & Utah CRD 60 & 49060 & Grand & 49019 & Mountain \\
\hline Utah & Utah CRD 70 & 49070 & Iron & 49021 & Mountain \\
\hline Utah & Utah CRD 50 & 49050 & Juab & 49023 & Mountain \\
\hline Utah & Utah CRD 70 & 49070 & Kane & 49025 & Mountain \\
\hline Utah & Utah CRD 50 & 49050 & Millard & 49027 & Mountain \\
\hline Utah & Utah CRD 10 & 49010 & Morgan & 49029 & Mountain \\
\hline Utah & Utah CRD 70 & 49070 & Piute & 49031 & Mountain \\
\hline Utah & Utah CRD 10 & 49010 & Rich & 49033 & Mountain \\
\hline Utah & Utah CRD 10 & 49010 & Salt Lake & 49035 & Mountain \\
\hline Utah & Utah CRD 60 & 49060 & San Juan & 49037 & Mountain \\
\hline Utah & Utah CRD 50 & 49050 & Sanpete & 49039 & Mountain \\
\hline Utah & Utah CRD 50 & 49050 & Sevier & 49041 & Mountain \\
\hline Utah & Utah CRD 60 & 49060 & Summit & 49043 & Mountain \\
\hline Utah & Utah CRD 10 & 49010 & Tooele & 49045 & Mountain \\
\hline Utah & Utah CRD 60 & 49060 & Uintah & 49047 & Mountain \\
\hline Utah & Utah CRD 50 & 49050 & Utah & 49049 & Mountain \\
\hline Utah & Utah CRD 60 & 49060 & Wasatch & 49051 & Mountain \\
\hline Utah & Utah CRD 70 & 49070 & Washington & 49053 & Mountain \\
\hline Utah & Utah CRD 70 & 49070 & Wayne & 49055 & Mountain \\
\hline Utah & Utah CRD 10 & 49010 & Weber & 49057 & Mountain \\
\hline Vermont & Vermont CRD 10 & 50010 & Addison & 50001 & Northeast \\
\hline
\end{tabular}


Table 1. Crop Reporting Districts (CRD) of the conterminous United States and associated counties.-Continued

[FIPS, Federal Information Processing Standard; USDA, U.S. Department of Agriculture]

\begin{tabular}{|c|c|c|c|c|c|}
\hline State & $\begin{array}{l}\text { Crop Reporting District } \\
\text { (CRD) }\end{array}$ & CRD Identifier & County Name & County FIPS & $\begin{array}{c}\text { USDA Farm Production } \\
\text { Region }\end{array}$ \\
\hline Vermont & Vermont CRD 10 & 50010 & Bennington & 50003 & Northeast \\
\hline Vermont & Vermont CRD 10 & 50010 & Caledonia & 50005 & Northeast \\
\hline Vermont & Vermont CRD 10 & 50010 & Chittenden & 50007 & Northeast \\
\hline Vermont & Vermont CRD 10 & 50010 & Essex & 50009 & Northeast \\
\hline Vermont & Vermont CRD 10 & 50010 & Franklin & 50011 & Northeast \\
\hline Vermont & Vermont CRD 10 & 50010 & Grand Isle & 50013 & Northeast \\
\hline Vermont & Vermont CRD 10 & 50010 & Lamoille & 50015 & Northeast \\
\hline Vermont & Vermont CRD 10 & 50010 & Orange & 50017 & Northeast \\
\hline Vermont & Vermont CRD 10 & 50010 & Orleans & 50019 & Northeast \\
\hline Vermont & Vermont CRD 10 & 50010 & Rutland & 50021 & Northeast \\
\hline Vermont & Vermont CRD 10 & 50010 & Washington & 50023 & Northeast \\
\hline Vermont & Vermont CRD 10 & 50010 & Windham & 50025 & Northeast \\
\hline Vermont & Vermont CRD 10 & 50010 & Windsor & 50027 & Northeast \\
\hline Virginia & Virginia CRD 60 & 51060 & Accomack & 51001 & Appalachian \\
\hline Virginia & Virginia CRD 50 & 51050 & Albemarle & 51003 & Appalachian \\
\hline Virginia & Virginia CRD 40 & 51040 & Alleghany & 51005 & Appalachian \\
\hline Virginia & Virginia CRD 50 & 51050 & Amelia & 51007 & Appalachian \\
\hline Virginia & Virginia CRD 50 & 51050 & Amherst & 51009 & Appalachian \\
\hline Virginia & Virginia CRD 50 & 51050 & Appomattox & 51011 & Appalachian \\
\hline Virginia & Virginia CRD 20 & 51020 & Arlington & 51013 & Appalachian \\
\hline Virginia & Virginia CRD 40 & 51040 & Augusta & 51015 & Appalachian \\
\hline Virginia & Virginia CRD 40 & 51040 & Bath & 51017 & Appalachian \\
\hline Virginia & Virginia CRD 50 & 51050 & Bedford & 51019 & Appalachian \\
\hline Virginia & Virginia CRD 70 & 51070 & Bland & 51021 & Appalachian \\
\hline Virginia & Virginia CRD 40 & 51040 & Botetourt & 51023 & Appalachian \\
\hline Virginia & Virginia CRD 90 & 51090 & Brunswick & 51025 & Appalachian \\
\hline Virginia & Virginia CRD 70 & 51070 & Buchanan & 51027 & Appalachian \\
\hline Virginia & Virginia CRD 50 & 51050 & Buckingham & 51029 & Appalachian \\
\hline Virginia & Virginia CRD 50 & 51050 & Campbell & 51031 & Appalachian \\
\hline Virginia & Virginia CRD 50 & 51050 & Caroline & 51033 & Appalachian \\
\hline Virginia & Virginia CRD 70 & 51070 & Carroll & 51035 & Appalachian \\
\hline Virginia & Virginia CRD 60 & 51060 & Charles City & 51036 & Appalachian \\
\hline Virginia & Virginia CRD 80 & 51080 & Charlotte & 51037 & Appalachian \\
\hline Virginia & Virginia CRD 50 & 51050 & Chesterfield & 51041 & Appalachian \\
\hline Virginia & Virginia CRD 20 & 51020 & Clarke & 51043 & Appalachian \\
\hline Virginia & Virginia CRD 40 & 51040 & Craig & 51045 & Appalachian \\
\hline Virginia & Virginia CRD 20 & 51020 & Culpeper & 51047 & Appalachian \\
\hline Virginia & Virginia CRD 50 & 51050 & Cumberland & 51049 & Appalachian \\
\hline Virginia & Virginia CRD 70 & 51070 & Dickenson & 51051 & Appalachian \\
\hline Virginia & Virginia CRD 90 & 51090 & Dinwiddie & 51053 & Appalachian \\
\hline Virginia & Virginia CRD 60 & 51060 & Essex & 51057 & Appalachian \\
\hline Virginia & Virginia CRD 20 & 51020 & Fairfax & 51059 & Appalachian \\
\hline
\end{tabular}


Table 1. Crop Reporting Districts (CRD) of the conterminous United States and associated counties. - Continued

[FIPS, Federal Information Processing Standard; USDA, U.S. Department of Agriculture]

\begin{tabular}{|c|c|c|c|c|c|}
\hline State & $\begin{array}{l}\text { Crop Reporting District } \\
\text { (CRD) }\end{array}$ & CRD Identifier & County Name & County FIPS & $\begin{array}{c}\text { USDA Farm Production } \\
\text { Region }\end{array}$ \\
\hline Virginia & Virginia CRD 20 & 51020 & Fauquier & 51061 & Appalachian \\
\hline Virginia & Virginia CRD 70 & 51070 & Floyd & 51063 & Appalachian \\
\hline Virginia & Virginia CRD 50 & 51050 & Fluvanna & 51065 & Appalachian \\
\hline Virginia & Virginia CRD 80 & 51080 & Franklin & 51067 & Appalachian \\
\hline Virginia & Virginia CRD 20 & 51020 & Frederick & 51069 & Appalachian \\
\hline Virginia & Virginia CRD 70 & 51070 & Giles & 51071 & Appalachian \\
\hline Virginia & Virginia CRD 60 & 51060 & Gloucester & 51073 & Appalachian \\
\hline Virginia & Virginia CRD 50 & 51050 & Goochland & 51075 & Appalachian \\
\hline Virginia & Virginia CRD 70 & 51070 & Grayson & 51077 & Appalachian \\
\hline Virginia & Virginia CRD 50 & 51050 & Greene & 51079 & Appalachian \\
\hline Virginia & Virginia CRD 90 & 51090 & Greensville & 51081 & Appalachian \\
\hline Virginia & Virginia CRD 80 & 51080 & Halifax & 51083 & Appalachian \\
\hline Virginia & Virginia CRD 50 & 51050 & Hanover & 51085 & Appalachian \\
\hline Virginia & Virginia CRD 50 & 51050 & Henrico & 51087 & Appalachian \\
\hline Virginia & Virginia CRD 80 & 51080 & Henry & 51089 & Appalachian \\
\hline Virginia & Virginia CRD 40 & 51040 & Highland & 51091 & Appalachian \\
\hline Virginia & Virginia CRD 90 & 51090 & Isle of Wight & 51093 & Appalachian \\
\hline Virginia & Virginia CRD 60 & 51060 & James City & 51095 & Appalachian \\
\hline Virginia & Virginia CRD 60 & 51060 & King and Queen & 51097 & Appalachian \\
\hline Virginia & Virginia CRD 60 & 51060 & King George & 51099 & Appalachian \\
\hline Virginia & Virginia CRD 60 & 51060 & King Eilliam & 51101 & Appalachian \\
\hline Virginia & Virginia CRD 60 & 51060 & Lancaster & 51103 & Appalachian \\
\hline Virginia & Virginia CRD 70 & 51070 & Lee & 51105 & Appalachian \\
\hline Virginia & Virginia CRD 20 & 51020 & Loudoun & 51107 & Appalachian \\
\hline Virginia & Virginia CRD 50 & 51050 & Louisa & 51109 & Appalachian \\
\hline Virginia & Virginia CRD 80 & 51080 & Lunenburg & 51111 & Appalachian \\
\hline Virginia & Virginia CRD 20 & 51020 & Madison & 51113 & Appalachian \\
\hline Virginia & Virginia CRD 60 & 51060 & Mathews & 51115 & Appalachian \\
\hline Virginia & Virginia CRD 90 & 51090 & Mecklenburg & 51117 & Appalachian \\
\hline Virginia & Virginia CRD 60 & 51060 & Middlesex & 51119 & Appalachian \\
\hline Virginia & Virginia CRD 70 & 51070 & Montgomery & 51121 & Appalachian \\
\hline Virginia & Virginia CRD 50 & 51050 & Nelson & 51125 & Appalachian \\
\hline Virginia & Virginia CRD 60 & 51060 & New Kent & 51127 & Appalachian \\
\hline Virginia & Virginia CRD 60 & 51060 & Northampton & 51131 & Appalachian \\
\hline Virginia & Virginia CRD 60 & 51060 & Northumberland & 51133 & Appalachian \\
\hline Virginia & Virginia CRD 80 & 51080 & Nottoway & 51135 & Appalachian \\
\hline Virginia & Virginia CRD 50 & 51050 & Orange & 51137 & Appalachian \\
\hline Virginia & Virginia CRD 20 & 51020 & Page & 51139 & Appalachian \\
\hline Virginia & Virginia CRD 80 & 51080 & Patrick & 51141 & Appalachian \\
\hline Virginia & Virginia CRD 80 & 51080 & Pittsylvania & 51143 & Appalachian \\
\hline Virginia & Virginia CRD 50 & 51050 & Powhatan & 51145 & Appalachian \\
\hline Virginia & Virginia CRD 50 & 51050 & Prince Edward & 51147 & Appalachian \\
\hline
\end{tabular}


Table 1. Crop Reporting Districts (CRD) of the conterminous United States and associated counties.-Continued

[FIPS, Federal Information Processing Standard; USDA, U.S. Department of Agriculture]

\begin{tabular}{|c|c|c|c|c|c|}
\hline State & $\begin{array}{l}\text { Crop Reporting District } \\
\text { (CRD) }\end{array}$ & CRD Identifier & County Name & County FIPS & $\begin{array}{c}\text { USDA Farm Production } \\
\text { Region }\end{array}$ \\
\hline Virginia & Virginia CRD 90 & 51090 & Prince George & 51149 & Appalachian \\
\hline Virginia & Virginia CRD 20 & 51020 & Prince William & 51153 & Appalachian \\
\hline Virginia & Virginia CRD 70 & 51070 & Pulaski & 51155 & Appalachian \\
\hline Virginia & Virginia CRD 20 & 51020 & Rappahannock & 51157 & Appalachian \\
\hline Virginia & Virginia CRD 60 & 51060 & Richmond & 51159 & Appalachian \\
\hline Virginia & Virginia CRD 40 & 51040 & Roanoke & 51161 & Appalachian \\
\hline Virginia & Virginia CRD 40 & 51040 & Rockbridge & 51163 & Appalachian \\
\hline Virginia & Virginia CRD 20 & 51020 & Rockingham & 51165 & Appalachian \\
\hline Virginia & Virginia CRD 70 & 51070 & Russell & 51167 & Appalachian \\
\hline Virginia & Virginia CRD 70 & 51070 & Scott & 51169 & Appalachian \\
\hline Virginia & Virginia CRD 20 & 51020 & Shenandoah & 51171 & Appalachian \\
\hline Virginia & Virginia CRD 70 & 51070 & Smyth & 51173 & Appalachian \\
\hline Virginia & Virginia CRD 90 & 51090 & Southampton & 51175 & Appalachian \\
\hline Virginia & Virginia CRD 50 & 51050 & Spotsylvania & 51177 & Appalachian \\
\hline Virginia & Virginia CRD 20 & 51020 & Stafford & 51179 & Appalachian \\
\hline Virginia & Virginia CRD 90 & 51090 & Surry & 51181 & Appalachian \\
\hline Virginia & Virginia CRD 90 & 51090 & Sussex & 51183 & Appalachian \\
\hline Virginia & Virginia CRD 70 & 51070 & Tazewell & 51185 & Appalachian \\
\hline Virginia & Virginia CRD 20 & 51020 & Warren & 51187 & Appalachian \\
\hline Virginia & Virginia CRD 70 & 51070 & Washington & 51191 & Appalachian \\
\hline Virginia & Virginia CRD 60 & 51060 & Westmoreland & 51193 & Appalachian \\
\hline Virginia & Virginia CRD 70 & 51070 & Wise & 51195 & Appalachian \\
\hline Virginia & Virginia CRD 70 & 51070 & Wythe & 51197 & Appalachian \\
\hline Virginia & Virginia CRD 60 & 51060 & York & 51199 & Appalachian \\
\hline Virginia & Virginia CRD 20 & 51020 & Alexandria & 51510 & Appalachian \\
\hline Virginia & Virginia CRD 50 & 51050 & Bedford & 51515 & Appalachian \\
\hline Virginia & Virginia CRD 70 & 51070 & Bristol & 51520 & Appalachian \\
\hline Virginia & Virginia CRD 40 & 51040 & Buena Vista & 51530 & Appalachian \\
\hline Virginia & Virginia CRD 50 & 51050 & Charlottesville & 51540 & Appalachian \\
\hline Virginia & Virginia CRD 90 & 51090 & Chesapeake & 51550 & Appalachian \\
\hline Virginia & Virginia CRD 40 & 51040 & Clifton Forge & 51560 & Appalachian \\
\hline Virginia & Virginia CRD 50 & 51050 & Colonial Heights & 51570 & Appalachian \\
\hline Virginia & Virginia CRD 40 & 51040 & Covington & 51580 & Appalachian \\
\hline Virginia & Virginia CRD 80 & 51080 & Danville & 51590 & Appalachian \\
\hline Virginia & Virginia CRD 90 & 51090 & Emporia & 51595 & Appalachian \\
\hline Virginia & Virginia CRD 20 & 51020 & Fairfax City & 51600 & Appalachian \\
\hline Virginia & Virginia CRD 20 & 51020 & Falls Church & 51610 & Appalachian \\
\hline Virginia & Virginia CRD 90 & 51090 & Franklin & 51620 & Appalachian \\
\hline Virginia & Virginia CRD 50 & 51050 & Fredericksburg & 51630 & Appalachian \\
\hline Virginia & Virginia CRD 70 & 51070 & Galax & 51640 & Appalachian \\
\hline Virginia & Virginia CRD 60 & 51060 & Hampton & 51650 & Appalachian \\
\hline Virginia & Virginia CRD 20 & 51020 & Harrisonburg & 51660 & Appalachian \\
\hline
\end{tabular}


Table 1. Crop Reporting Districts (CRD) of the conterminous United States and associated counties.-Continued

[FIPS, Federal Information Processing Standard; USDA, U.S. Department of Agriculture]

\begin{tabular}{|c|c|c|c|c|c|}
\hline State & $\begin{array}{l}\text { Crop Reporting District } \\
\text { (CRD) }\end{array}$ & CRD Identifier & County Name & County FIPS & $\begin{array}{c}\text { USDA Farm Production } \\
\text { Region }\end{array}$ \\
\hline Virginia & Virginia CRD 90 & 51090 & Hopewell & 51670 & Appalachian \\
\hline Virginia & Virginia CRD 40 & 51040 & Lexington & 51678 & Appalachian \\
\hline Virginia & Virginia CRD 50 & 51050 & Lynchburg & 51680 & Appalachian \\
\hline Virginia & Virginia CRD 20 & 51020 & Manassas & 51683 & Appalachian \\
\hline Virginia & Virginia CRD 20 & 51020 & Manassas Park & 51685 & Appalachian \\
\hline Virginia & Virginia CRD 80 & 51080 & Martinsville & 51690 & Appalachian \\
\hline Virginia & Virginia CRD 60 & 51060 & Newport News & 51700 & Appalachian \\
\hline Virginia & Virginia CRD 90 & 51090 & Norfolk & 51710 & Appalachian \\
\hline Virginia & Virginia CRD 70 & 51070 & Norton & 51720 & Appalachian \\
\hline Virginia & Virginia CRD 90 & 51090 & Petersburg & 51730 & Appalachian \\
\hline Virginia & Virginia CRD 60 & 51060 & Poquoson & 51735 & Appalachian \\
\hline Virginia & Virginia CRD 90 & 51090 & Portsmouth & 51740 & Appalachian \\
\hline Virginia & Virginia CRD 70 & 51070 & Radford & 51750 & Appalachian \\
\hline Virginia & Virginia CRD 50 & 51050 & Richmond & 51760 & Appalachian \\
\hline Virginia & Virginia CRD 40 & 51040 & Roanoke & 51770 & Appalachian \\
\hline Virginia & Virginia CRD 40 & 51040 & Salem & 51775 & Appalachian \\
\hline Virginia & Virginia CRD 40 & 51040 & Staunton & 51790 & Appalachian \\
\hline Virginia & Virginia CRD 90 & 51090 & Suffolk & 51800 & Appalachian \\
\hline Virginia & Virginia CRD 90 & 51090 & Virginia Beach & 51810 & Appalachian \\
\hline Virginia & Virginia CRD 40 & 51040 & Waynesboro & 51820 & Appalachian \\
\hline Virginia & Virginia CRD 60 & 51060 & Williamsburg & 51830 & Appalachian \\
\hline Virginia & Virginia CRD 20 & 51020 & Winchester & 51840 & Appalachian \\
\hline Washington & Washington CRD 50 & 53050 & Adams & 53001 & Pacific \\
\hline Washington & Washington CRD 90 & 53090 & Asotin & 53003 & Pacific \\
\hline Washington & Washington CRD 20 & 53020 & Benton & 53005 & Pacific \\
\hline Washington & Washington CRD 20 & 53020 & Chelan & 53007 & Pacific \\
\hline Washington & Washington CRD 10 & 53010 & Clallam & 53009 & Pacific \\
\hline Washington & Washington CRD 10 & 53010 & Clark & 53011 & Pacific \\
\hline Washington & Washington CRD 90 & 53090 & Columbia & 53013 & Pacific \\
\hline Washington & Washington CRD 10 & 53010 & Cowlitz & 53015 & Pacific \\
\hline Washington & Washington CRD 50 & 53050 & Douglas & 53017 & Pacific \\
\hline Washington & Washington CRD 30 & 53030 & Ferry & 53019 & Pacific \\
\hline Washington & Washington CRD 50 & 53050 & Franklin & 53021 & Pacific \\
\hline Washington & Washington CRD 90 & 53090 & Garfield & 53023 & Pacific \\
\hline Washington & Washington CRD 50 & 53050 & Grant & 53025 & Pacific \\
\hline Washington & Washington CRD 10 & 53010 & Grays Harbor & 53027 & Pacific \\
\hline Washington & Washington CRD 10 & 53010 & Island & 53029 & Pacific \\
\hline Washington & Washington CRD 10 & 53010 & Jefferson & 53031 & Pacific \\
\hline Washington & Washington CRD 10 & 53010 & King & 53033 & Pacific \\
\hline Washington & Washington CRD 10 & 53010 & Kitsap & 53035 & Pacific \\
\hline Washington & Washington CRD 20 & 53020 & Kittitas & 53037 & Pacific \\
\hline Washington & Washington CRD 20 & 53020 & Klickitat & 53039 & Pacific \\
\hline
\end{tabular}


Table 1. Crop Reporting Districts (CRD) of the conterminous United States and associated counties. - Continued

[FIPS, Federal Information Processing Standard; USDA, U.S. Department of Agriculture]

\begin{tabular}{|c|c|c|c|c|c|}
\hline State & $\begin{array}{l}\text { Crop Reporting District } \\
\text { (CRD) }\end{array}$ & CRD Identifier & County Name & County FIPS & $\begin{array}{c}\text { USDA Farm Production } \\
\text { Region }\end{array}$ \\
\hline Washington & Washington CRD 10 & 53010 & Lewis & 53041 & Pacific \\
\hline Washington & Washington CRD 50 & 53050 & Lincoln & 53043 & Pacific \\
\hline Washington & Washington CRD 10 & 53010 & Mason & 53045 & Pacific \\
\hline Washington & Washington CRD 20 & 53020 & Okanogan & 53047 & Pacific \\
\hline Washington & Washington CRD 10 & 53010 & Pacific & 53049 & Pacific \\
\hline Washington & Washington CRD 30 & 53030 & Pend Oreille & 53051 & Pacific \\
\hline Washington & Washington CRD 10 & 53010 & Pierce & 53053 & Pacific \\
\hline Washington & Washington CRD 10 & 53010 & San juan & 53055 & Pacific \\
\hline Washington & Washington CRD 10 & 53010 & Skagit & 53057 & Pacific \\
\hline Washington & Washington CRD 10 & 53010 & Skamania & 53059 & Pacific \\
\hline Washington & Washington CRD 10 & 53010 & Snohomish & 53061 & Pacific \\
\hline Washington & Washington CRD 30 & 53030 & Spokane & 53063 & Pacific \\
\hline Washington & Washington CRD 30 & 53030 & Stevens & 53065 & Pacific \\
\hline Washington & Washington CRD 10 & 53010 & Thurston & 53067 & Pacific \\
\hline Washington & Washington CRD 10 & 53010 & Wahkiakum & 53069 & Pacific \\
\hline Washington & Washington CRD 90 & 53090 & Walla Walla & 53071 & Pacific \\
\hline Washington & Washington CRD 10 & 53010 & Whatcom & 53073 & Pacific \\
\hline Washington & Washington CRD 90 & 53090 & Whitman & 53075 & Pacific \\
\hline Washington & Washington CRD 20 & 53020 & Yakima & 53077 & Pacific \\
\hline West Virginia & West Virginia CRD 20 & 54020 & Barbour & 54001 & Appalachian \\
\hline West Virginia & West Virginia CRD 60 & 54060 & Berkeley & 54003 & Appalachian \\
\hline West Virginia & West Virginia CRD 40 & 54040 & Boone & 54005 & Appalachian \\
\hline West Virginia & West Virginia CRD 40 & 54040 & Braxton & 54007 & Appalachian \\
\hline West Virginia & West Virginia CRD 20 & 54020 & Brooke & 54009 & Appalachian \\
\hline West Virginia & West Virginia CRD 40 & 54040 & Cabell & 54011 & Appalachian \\
\hline West Virginia & West Virginia CRD 40 & 54040 & Calhoun & 54013 & Appalachian \\
\hline West Virginia & West Virginia CRD 40 & 54040 & Clay & 54015 & Appalachian \\
\hline West Virginia & West Virginia CRD 20 & 54020 & Doddridge & 54017 & Appalachian \\
\hline West Virginia & West Virginia CRD 40 & 54040 & Fayette & 54019 & Appalachian \\
\hline West Virginia & West Virginia CRD 40 & 54040 & Gilmer & 54021 & Appalachian \\
\hline West Virginia & West Virginia CRD 60 & 54060 & Grant & 54023 & Appalachian \\
\hline West Virginia & West Virginia CRD 60 & 54060 & Greenbrier & 54025 & Appalachian \\
\hline West Virginia & West Virginia CRD 60 & 54060 & Hampshire & 54027 & Appalachian \\
\hline West Virginia & West Virginia CRD 20 & 54020 & Hancock & 54029 & Appalachian \\
\hline West Virginia & West Virginia CRD 60 & 54060 & Hardy & 54031 & Appalachian \\
\hline West Virginia & West Virginia CRD 20 & 54020 & Harrison & 54033 & Appalachian \\
\hline West Virginia & West Virginia CRD 40 & 54040 & Jackson & 54035 & Appalachian \\
\hline West Virginia & West Virginia CRD 60 & 54060 & Jefferson & 54037 & Appalachian \\
\hline West Virginia & West Virginia CRD 40 & 54040 & Kanawha & 54039 & Appalachian \\
\hline West Virginia & West Virginia CRD 20 & 54020 & Lewis & 54041 & Appalachian \\
\hline West Virginia & West Virginia CRD 40 & 54040 & Lincoln & 54043 & Appalachian \\
\hline West Virginia & West Virginia CRD 40 & 54040 & Logan & 54045 & Appalachian \\
\hline
\end{tabular}


Table 1. Crop Reporting Districts (CRD) of the conterminous United States and associated counties.-Continued

[FIPS, Federal Information Processing Standard; USDA, U.S. Department of Agriculture]

\begin{tabular}{|c|c|c|c|c|c|}
\hline State & $\begin{array}{l}\text { Crop Reporting District } \\
\text { (CRD) }\end{array}$ & CRD Identifier & County Name & County FIPS & $\begin{array}{c}\text { USDA Farm Production } \\
\text { Region }\end{array}$ \\
\hline West Virginia & West Virginia CRD 40 & 54040 & McDowell & 54047 & Appalachian \\
\hline West Virginia & West Virginia CRD 20 & 54020 & Marion & 54049 & Appalachian \\
\hline West Virginia & West Virginia CRD 20 & 54020 & Marshall & 54051 & Appalachian \\
\hline West Virginia & West Virginia CRD 40 & 54040 & Mason & 54053 & Appalachian \\
\hline West Virginia & West Virginia CRD 40 & 54040 & Mercer & 54055 & Appalachian \\
\hline West Virginia & West Virginia CRD 60 & 54060 & Mineral & 54057 & Appalachian \\
\hline West Virginia & West Virginia CRD 40 & 54040 & Mingo & 54059 & Appalachian \\
\hline West Virginia & West Virginia CRD 20 & 54020 & Monongalia & 54061 & Appalachian \\
\hline West Virginia & West Virginia CRD 60 & 54060 & Monroe & 54063 & Appalachian \\
\hline West Virginia & West Virginia CRD 60 & 54060 & Morgan & 54065 & Appalachian \\
\hline West Virginia & West Virginia CRD 40 & 54040 & Nicholas & 54067 & Appalachian \\
\hline West Virginia & West Virginia CRD 20 & 54020 & Ohio & 54069 & Appalachian \\
\hline West Virginia & West Virginia CRD 60 & 54060 & Pendleton & 54071 & Appalachian \\
\hline West Virginia & West Virginia CRD 20 & 54020 & Pleasants & 54073 & Appalachian \\
\hline West Virginia & West Virginia CRD 60 & 54060 & Pocahontas & 54075 & Appalachian \\
\hline West Virginia & West Virginia CRD 20 & 54020 & Preston & 54077 & Appalachian \\
\hline West Virginia & West Virginia CRD 40 & 54040 & Putnam & 54079 & Appalachian \\
\hline West Virginia & West Virginia CRD 40 & 54040 & Raleigh & 54081 & Appalachian \\
\hline West Virginia & West Virginia CRD 60 & 54060 & Randolph & 54083 & Appalachian \\
\hline West Virginia & West Virginia CRD 20 & 54020 & Ritchie & 54085 & Appalachian \\
\hline West Virginia & West Virginia CRD 40 & 54040 & Roane & 54087 & Appalachian \\
\hline West Virginia & West Virginia CRD 60 & 54060 & Summers & 54089 & Appalachian \\
\hline West Virginia & West Virginia CRD 20 & 54020 & Taylor & 54091 & Appalachian \\
\hline West Virginia & West Virginia CRD 60 & 54060 & Tucker & 54093 & Appalachian \\
\hline West Virginia & West Virginia CRD 20 & 54020 & Tyler & 54095 & Appalachian \\
\hline West Virginia & West Virginia CRD 20 & 54020 & Upshur & 54097 & Appalachian \\
\hline West Virginia & West Virginia CRD 40 & 54040 & Wayne & 54099 & Appalachian \\
\hline West Virginia & West Virginia CRD 40 & 54040 & Webster & 54101 & Appalachian \\
\hline West Virginia & West Virginia CRD 20 & 54020 & Wetzel & 54103 & Appalachian \\
\hline West Virginia & West Virginia CRD 40 & 54040 & Wirt & 54105 & Appalachian \\
\hline West Virginia & West Virginia CRD 20 & 54020 & Wood & 54107 & Appalachian \\
\hline West Virginia & West Virginia CRD 40 & 54040 & Wyoming & 54109 & Appalachian \\
\hline Wisconsin & Wisconsin CRD 50 & 55050 & Adams & 55001 & Lake-states \\
\hline Wisconsin & Wisconsin CRD 20 & 55020 & Ashland & 55003 & Lake-states \\
\hline Wisconsin & Wisconsin CRD 10 & 55010 & Barron & 55005 & Lake-states \\
\hline Wisconsin & Wisconsin CRD 10 & 55010 & Bayfield & 55007 & Lake-states \\
\hline Wisconsin & Wisconsin CRD 60 & 55060 & Brown & 55009 & Lake-states \\
\hline Wisconsin & Wisconsin CRD 40 & 55040 & Buffalo & 55011 & Lake-states \\
\hline Wisconsin & Wisconsin CRD 10 & 55010 & Burnett & 55013 & Lake-states \\
\hline Wisconsin & Wisconsin CRD 60 & 55060 & Calumet & 55015 & Lake-states \\
\hline Wisconsin & Wisconsin CRD 10 & 55010 & Chippewa & 55017 & Lake-states \\
\hline Wisconsin & Wisconsin CRD 20 & 55020 & Clark & 55019 & Lake-states \\
\hline
\end{tabular}


Table 1. Crop Reporting Districts (CRD) of the conterminous United States and associated counties. - Continued

[FIPS, Federal Information Processing Standard; USDA, U.S. Department of Agriculture]

\begin{tabular}{|c|c|c|c|c|c|}
\hline State & $\begin{array}{l}\text { Crop Reporting District } \\
\text { (CRD) }\end{array}$ & CRD Identifier & County Name & County FIPS & $\begin{array}{l}\text { USDA Farm Production } \\
\text { Region }\end{array}$ \\
\hline Wisconsin & Wisconsin CRD 80 & 55080 & Columbia & 55021 & Lake-states \\
\hline Wisconsin & Wisconsin CRD 70 & 55070 & Crawford & 55023 & Lake-states \\
\hline Wisconsin & Wisconsin CRD 80 & 55080 & Dane & 55025 & Lake-states \\
\hline Wisconsin & Wisconsin CRD 80 & 55080 & Dodge & 55027 & Lake-states \\
\hline Wisconsin & Wisconsin CRD 60 & 55060 & Door & 55029 & Lake-states \\
\hline Wisconsin & Wisconsin CRD 10 & 55010 & Douglas & 55031 & Lake-states \\
\hline Wisconsin & Wisconsin CRD 40 & 55040 & Dunn & 55033 & Lake-states \\
\hline Wisconsin & Wisconsin CRD 40 & 55040 & Eau Claire & 55035 & Lake-states \\
\hline Wisconsin & Wisconsin CRD 30 & 55030 & Florence & 55037 & Lake-states \\
\hline Wisconsin & Wisconsin CRD 60 & 55060 & Fond du Lac & 55039 & Lake-states \\
\hline Wisconsin & Wisconsin CRD 30 & 55030 & Forest & 55041 & Lake-states \\
\hline Wisconsin & Wisconsin CRD 70 & 55070 & Grant & 55043 & Lake-states \\
\hline Wisconsin & Wisconsin CRD 80 & 55080 & Green & 55045 & Lake-states \\
\hline Wisconsin & Wisconsin CRD 50 & 55050 & Green Lake & 55047 & Lake-states \\
\hline Wisconsin & Wisconsin CRD 70 & 55070 & Iowa & 55049 & Lake-states \\
\hline Wisconsin & Wisconsin CRD 20 & 55020 & Iron & 55051 & Lake-states \\
\hline Wisconsin & Wisconsin CRD 40 & 55040 & Jackson & 55053 & Lake-states \\
\hline Wisconsin & Wisconsin CRD 80 & 55080 & Jefferson & 55055 & Lake-states \\
\hline Wisconsin & Wisconsin CRD 50 & 55050 & Juneau & 55057 & Lake-states \\
\hline Wisconsin & Wisconsin CRD 90 & 55090 & Kenosha & 55059 & Lake-states \\
\hline Wisconsin & Wisconsin CRD 60 & 55060 & Kewaunee & 55061 & Lake-states \\
\hline Wisconsin & Wisconsin CRD 40 & 55040 & La Crosse & 55063 & Lake-states \\
\hline Wisconsin & Wisconsin CRD 70 & 55070 & Lafayette & 55065 & Lake-states \\
\hline Wisconsin & Wisconsin CRD 30 & 55030 & Langlade & 55067 & Lake-states \\
\hline Wisconsin & Wisconsin CRD 20 & 55020 & Lincoln & 55069 & Lake-states \\
\hline Wisconsin & Wisconsin CRD 60 & 55060 & Manitowoc & 55071 & Lake-states \\
\hline Wisconsin & Wisconsin CRD 20 & 55020 & Marathon & 55073 & Lake-states \\
\hline Wisconsin & Wisconsin CRD 30 & 55030 & Marinette & 55075 & Lake-states \\
\hline Wisconsin & Wisconsin CRD 50 & 55050 & Marquette & 55077 & Lake-states \\
\hline Wisconsin & Wisconsin CRD 30 & 55030 & Menominee & 55078 & Lake-states \\
\hline Wisconsin & Wisconsin CRD 90 & 55090 & Milwaukee & 55079 & Lake-states \\
\hline Wisconsin & Wisconsin CRD 40 & 55040 & Monroe & 55081 & Lake-states \\
\hline Wisconsin & Wisconsin CRD 30 & 55030 & Oconto & 55083 & Lake-states \\
\hline Wisconsin & Wisconsin CRD 20 & 55020 & Oneida & 55085 & Lake-states \\
\hline Wisconsin & Wisconsin CRD 60 & 55060 & Outagamie & 55087 & Lake-states \\
\hline Wisconsin & Wisconsin CRD 90 & 55090 & Ozaukee & 55089 & Lake-states \\
\hline Wisconsin & Wisconsin CRD 40 & 55040 & Pepin & 55091 & Lake-states \\
\hline Wisconsin & Wisconsin CRD 40 & 55040 & Pierce & 55093 & Lake-states \\
\hline Wisconsin & Wisconsin CRD 10 & 55010 & Polk & 55095 & Lake-states \\
\hline Wisconsin & Wisconsin CRD 50 & 55050 & Portage & 55097 & Lake-states \\
\hline Wisconsin & Wisconsin CRD 20 & 55020 & Price & 55099 & Lake-states \\
\hline Wisconsin & Wisconsin CRD 90 & 55090 & Racine & 55101 & Lake-states \\
\hline
\end{tabular}


Table 1. Crop Reporting Districts (CRD) of the conterminous United States and associated counties.-Continued

[FIPS, Federal Information Processing Standard; USDA, U.S. Department of Agriculture]

\begin{tabular}{|c|c|c|c|c|c|}
\hline State & $\begin{array}{l}\text { Crop Reporting District } \\
\text { (CRD) }\end{array}$ & CRD Identifier & County Name & County FIPS & $\begin{array}{l}\text { USDA Farm Production } \\
\text { Region }\end{array}$ \\
\hline Wisconsin & Wisconsin CRD 70 & 55070 & Richland & 55103 & Lake-states \\
\hline Wisconsin & Wisconsin CRD 80 & 55080 & Rock & 55105 & Lake-states \\
\hline Wisconsin & Wisconsin CRD 10 & 55010 & Rusk & 55107 & Lake-states \\
\hline Wisconsin & Wisconsin CRD 40 & 55040 & St. Croix & 55109 & Lake-states \\
\hline Wisconsin & Wisconsin CRD 70 & 55070 & Sauk & 55111 & Lake-states \\
\hline Wisconsin & Wisconsin CRD 10 & 55010 & Sawyer & 55113 & Lake-states \\
\hline Wisconsin & Wisconsin CRD 30 & 55030 & Shawano & 55115 & Lake-states \\
\hline Wisconsin & Wisconsin CRD 60 & 55060 & Sheboygan & 55117 & Lake-states \\
\hline Wisconsin & Wisconsin CRD 20 & 55020 & Taylor & 55119 & Lake-states \\
\hline Wisconsin & Wisconsin CRD 40 & 55040 & Trempealeau & 55121 & Lake-states \\
\hline Wisconsin & Wisconsin CRD 70 & 55070 & Vernon & 55123 & Lake-states \\
\hline Wisconsin & Wisconsin CRD 20 & 55020 & Vilas & 55125 & Lake-states \\
\hline Wisconsin & Wisconsin CRD 90 & 55090 & Walworth & 55127 & Lake-states \\
\hline Wisconsin & Wisconsin CRD 10 & 55010 & Washburn & 55129 & Lake-states \\
\hline Wisconsin & Wisconsin CRD 90 & 55090 & Washington & 55131 & Lake-states \\
\hline Wisconsin & Wisconsin CRD 90 & 55090 & Waukesha & 55133 & Lake-states \\
\hline Wisconsin & Wisconsin CRD 50 & 55050 & Waupaca & 55135 & Lake-states \\
\hline Wisconsin & Wisconsin CRD 50 & 55050 & Waushara & 55137 & Lake-states \\
\hline Wisconsin & Wisconsin CRD 60 & 55060 & Winnebago & 55139 & Lake-states \\
\hline Wisconsin & Wisconsin CRD 50 & 55050 & Wood & 55141 & Lake-states \\
\hline Wyoming & Wyoming CRD 40 & 56040 & Albany & 56001 & Mountain \\
\hline Wyoming & Wyoming CRD 10 & 56010 & Big Horn & 56003 & Mountain \\
\hline Wyoming & Wyoming CRD 20 & 56020 & Campbell & 56005 & Mountain \\
\hline Wyoming & Wyoming CRD 40 & 56040 & Carbon & 56007 & Mountain \\
\hline Wyoming & Wyoming CRD 50 & 56050 & Converse & 56009 & Mountain \\
\hline Wyoming & Wyoming CRD 20 & 56020 & Crook & 56011 & Mountain \\
\hline Wyoming & Wyoming CRD 10 & 56010 & Fremont & 56013 & Mountain \\
\hline Wyoming & Wyoming CRD 50 & 56050 & Goshen & 56015 & Mountain \\
\hline Wyoming & Wyoming CRD 10 & 56010 & Hot Springs & 56017 & Mountain \\
\hline Wyoming & Wyoming CRD 20 & 56020 & Johnson & 56019 & Mountain \\
\hline Wyoming & Wyoming CRD 50 & 56050 & Laramie & 56021 & Mountain \\
\hline Wyoming & Wyoming CRD 30 & 56030 & Lincoln & 56023 & Mountain \\
\hline Wyoming & Wyoming CRD 40 & 56040 & Natrona & 56025 & Mountain \\
\hline Wyoming & Wyoming CRD 50 & 56050 & Niobrara & 56027 & Mountain \\
\hline Wyoming & Wyoming CRD 10 & 56010 & Park & 56029 & Mountain \\
\hline Wyoming & Wyoming CRD 50 & 56050 & Platte & 56031 & Mountain \\
\hline Wyoming & Wyoming CRD 20 & 56020 & Sheridan & 56033 & Mountain \\
\hline Wyoming & Wyoming CRD 30 & 56030 & Sublette & 56035 & Mountain \\
\hline Wyoming & Wyoming CRD 40 & 56040 & Sweetwater & 56037 & Mountain \\
\hline Wyoming & Wyoming CRD 30 & 56030 & Teton & 56039 & Mountain \\
\hline Wyoming & Wyoming CRD 30 & 56030 & Uinta & 56041 & Mountain \\
\hline Wyoming & Wyoming CRD 10 & 56010 & Washakie & 56043 & Mountain \\
\hline Wyoming & Wyoming CRD 20 & 56020 & Weston & 56045 & Mountain \\
\hline
\end{tabular}


Table 2. Relation of Doane Marketing Research-Kynetec (DMRK) crop categories (not including California) to Census of Agriculture and National Agriculture Statistics Service crop categories used in the atrazine-use estimation process.

[USDA, U.S. Department of Agriculture]

\begin{tabular}{|c|c|c|}
\hline $\begin{array}{l}\text { Crop atrazine estimates reported in } \\
\text { DMRK-AgroTrak database }\end{array}$ & $\begin{array}{l}\text { Census of Agriculture } \\
\text { crop categories }\end{array}$ & $\begin{array}{l}\text { USDA National Agriculture Statistics } \\
\text { Service crop categories }\end{array}$ \\
\hline Alfalfa & Alfalfa seed, alfalfa hay & Alfalfa hay \\
\hline Barley & Barley for grain & Barley, all \\
\hline Beans/peas, vegetables & Snap beans, green peas (excluding southern) & Snap beans for processing \\
\hline Corn & $\begin{array}{l}\text { Corn for silage or greenchop, corn for grain or seed, } \\
\text { corn cut for dry fodder, hogged or grazed }\end{array}$ & $\begin{array}{l}\text { Corn for grain, corn for silage, corn for } \\
\text { forage }\end{array}$ \\
\hline Cotton & Cotton, all & Cotton, American, pima, cotton upland \\
\hline Cropland for pasture & Cropland used only for pasture or grazing & \\
\hline Lots/farmstead, other & $\begin{array}{l}\text { Land in farmsteads, buildings, livestock facilities, } \\
\text { ponds, roads, wasteland, etc. }\end{array}$ & \\
\hline Oats/rye & Oats for grain, rye for grain & Oats \\
\hline Oranges & Oranges & \\
\hline $\begin{array}{l}\text { Pasture/rangeland, or pasture/ } \\
\text { rangeland, other }\end{array}$ & $\begin{array}{l}\text { Pasture land and rangeland, other than cropland and } \\
\text { woodland pastured }\end{array}$ & \\
\hline Pasture land & Pasture land, all types & \\
\hline Sorghum & Sorghum- including grain, syrup, silage, and hogged & Sorghum for grain and sorghum for silage \\
\hline Soybeans & Soybeans for beans & Soybeans \\
\hline
\end{tabular}


Table 3. Mapping of California Department of Pesticide Regulation (DPR) crops to EPest crops and associated 1992 and 2001 National Land Cover Dataset (NLCD) crop categories reported in the county atrazine-use database.

[-, not reported]

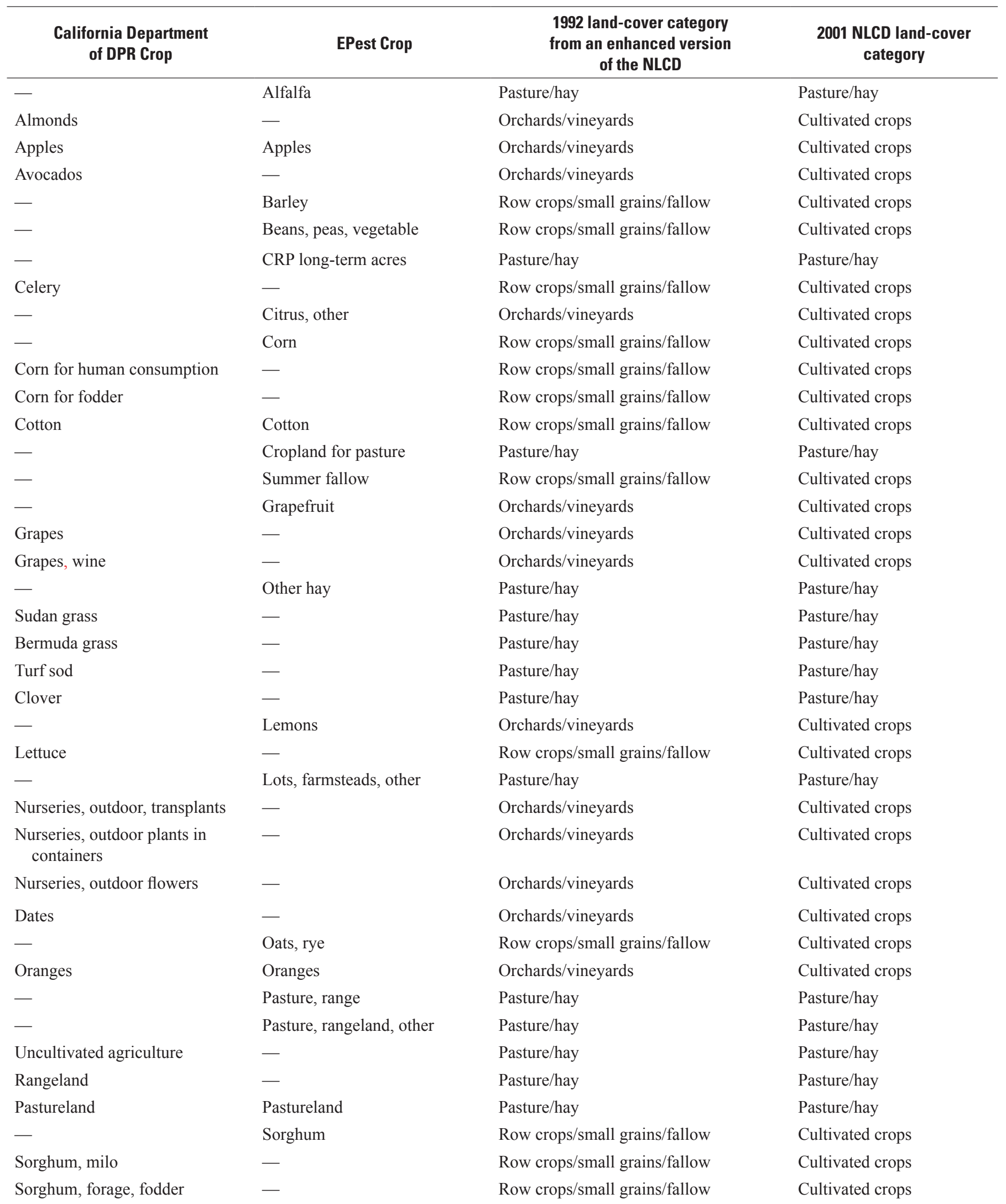


Table 3. Mapping of California Department of Pesticide Regulation (DPR) crops to EPest crops and associated 1992 and 2001 National Land Cover Dataset (NLCD) crop categories reported in the county atrazine-use database.-Continued

$[-$, not reported $]$

\begin{tabular}{llll}
\hline $\begin{array}{c}\text { California Department } \\
\text { of DPR Crop }\end{array}$ & \multicolumn{1}{c}{ EPest Crop } & \multicolumn{1}{c}{$\begin{array}{c}\text { 1992 land-cover category } \\
\text { from an enhanced version } \\
\text { of the NLCD }\end{array}$} & $\begin{array}{c}\text { 2001 NLCD land-cover } \\
\text { category }\end{array}$ \\
\hline- & Soybeans & Row crops/small grains/fallow & Cultivated crops \\
Olive & - & Orchards/vineyards & Cultivated crops \\
Strawberries & - & Orchards/vineyards & Cultivated crops \\
Sugarcane & Sugarcane & Row crops/small grains/fallow & Cultivated crops \\
- & Sweet corn & Row crops/small grains/fallow & Cultivated crops \\
Tomato & - & Row crops/small grains/fallow & Cultivated crops \\
Walnut & - & Orchards/vineyards & Cultivated crops \\
- & Wheat, spring & Row crops/small grains/fallow & Cultivated crops \\
- & Wheat, winter & Row crops/small grains/fallow & Cultivated crops \\
Wheat & - & Row crops/small grains/fallow & Cultivated crops \\
\hline
\end{tabular}


Appendix: Table A1. Annual atrazine-use intensity estimates for watersheds used to develop the Watershed Regressions for Pesticides model, 1992 through 1999.

[kg/km², kilogram per square kilometer; - , no estimate]

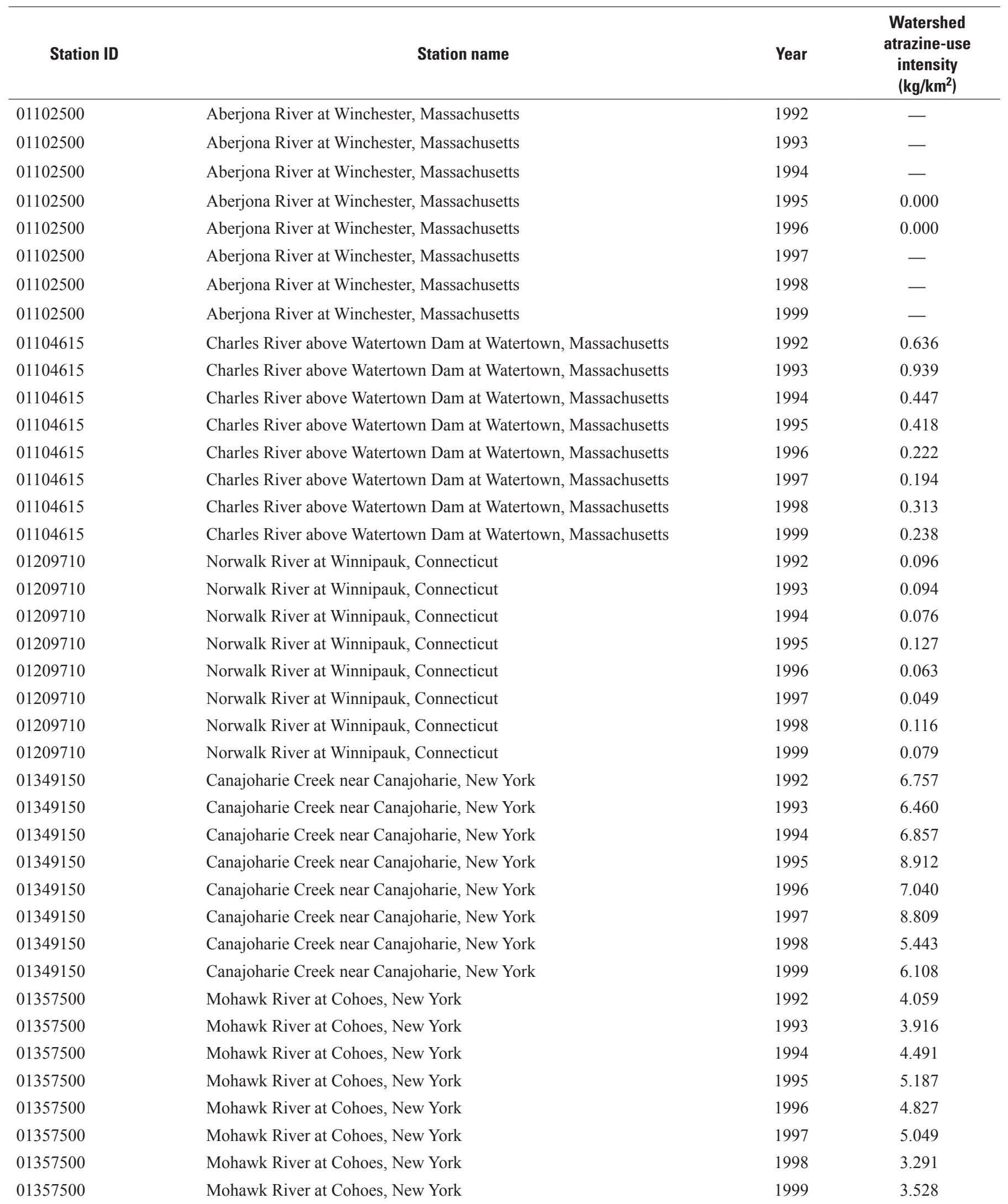


Appendix: Table A1. Annual atrazine-use intensity estimates for watersheds used to develop the Watershed Regressions for Pesticides model, 1992 through 1999.-Continued

$\left[\mathrm{kg} / \mathrm{km}^{2}\right.$, kilogram per square kilometer; - , no estimate $]$

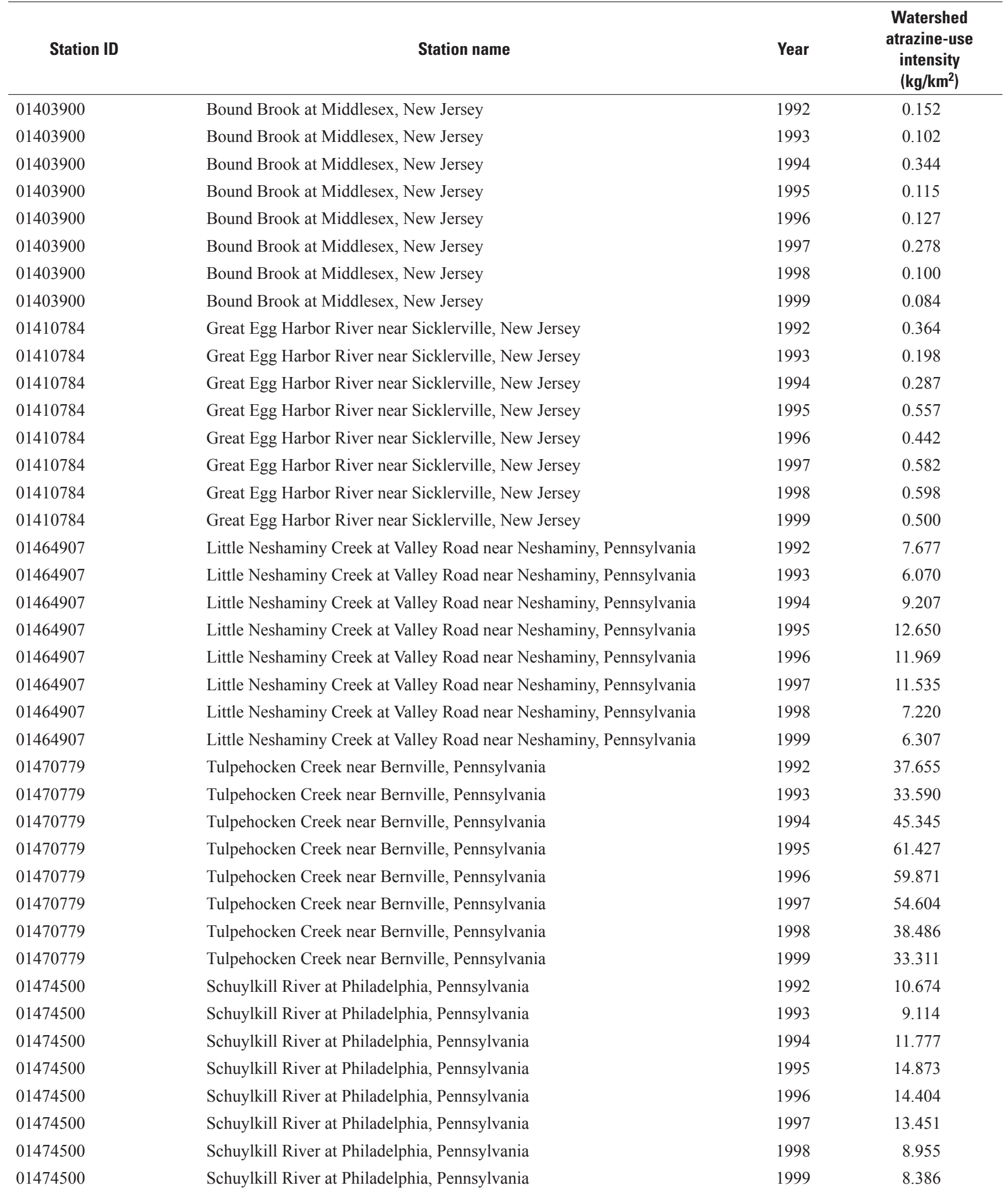


Appendix: Table A1. Annual atrazine-use intensity estimates for watersheds used to develop the Watershed Regressions for Pesticides model, 1992 through 1999._- Continued

$\left[\mathrm{kg} / \mathrm{km}^{2}\right.$, kilogram per square kilometer; —, no estimate $]$

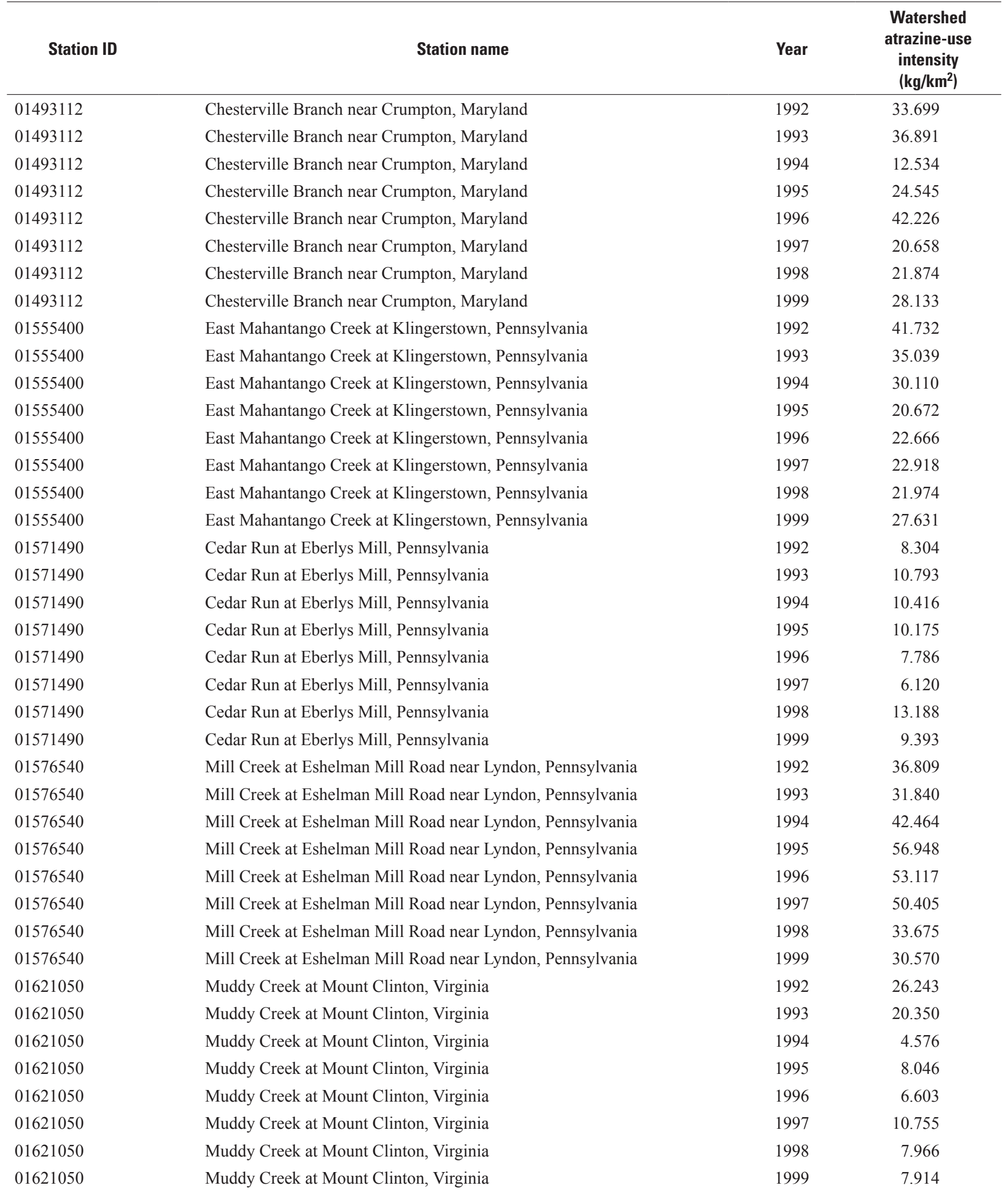


Appendix: Table A1. Annual atrazine-use intensity estimates for watersheds used to develop the Watershed Regressions for Pesticides model, 1992 through 1999. - Continued

$\left[\mathrm{kg} / \mathrm{km}^{2}\right.$, kilogram per square kilometer; - , no estimate $]$

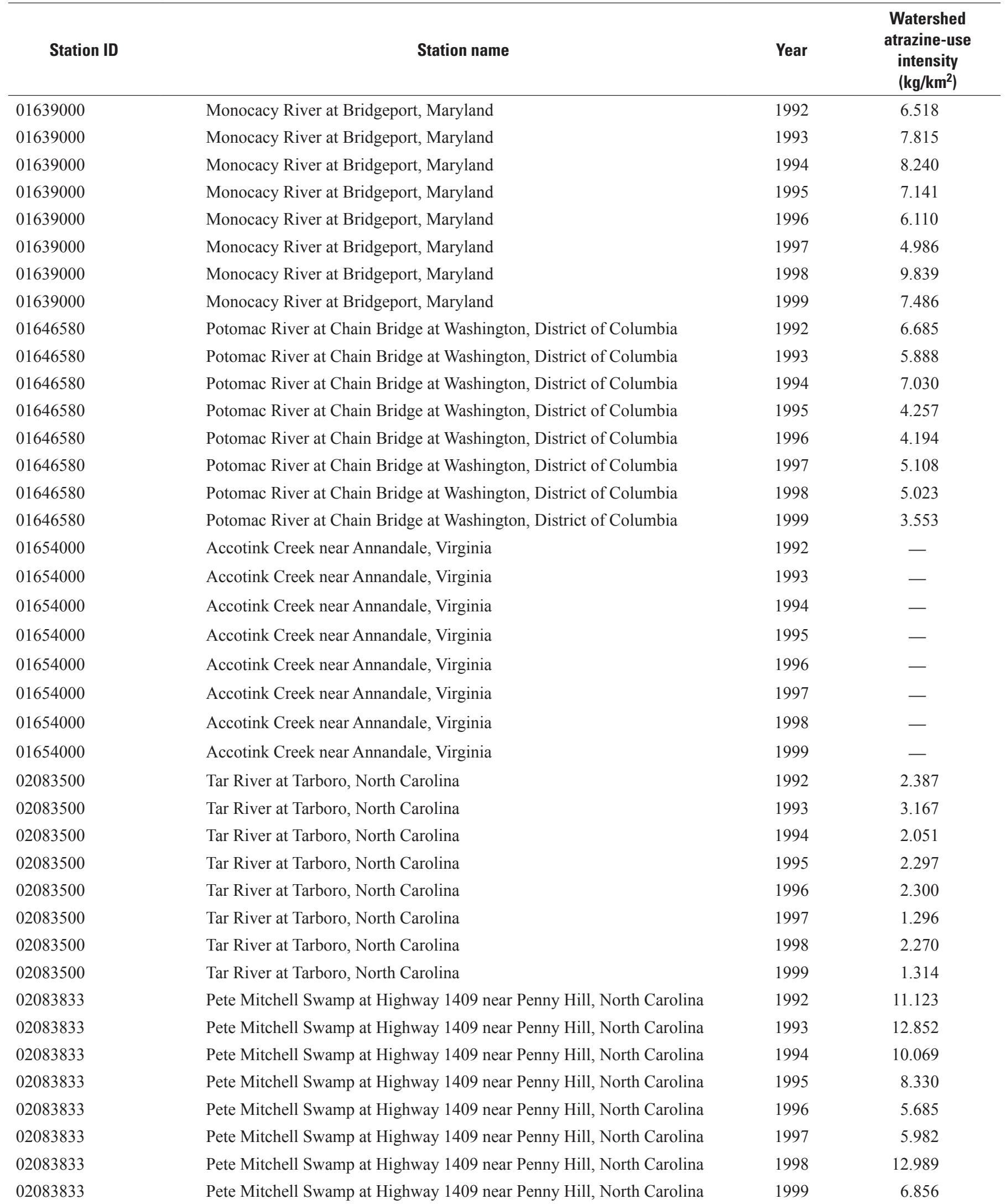


Appendix: Table A1. Annual atrazine-use intensity estimates for watersheds used to develop the Watershed Regressions for Pesticides model, 1992 through 1999._- Continued

$\left[\mathrm{kg} / \mathrm{km}^{2}\right.$, kilogram per square kilometer; —, no estimate $]$

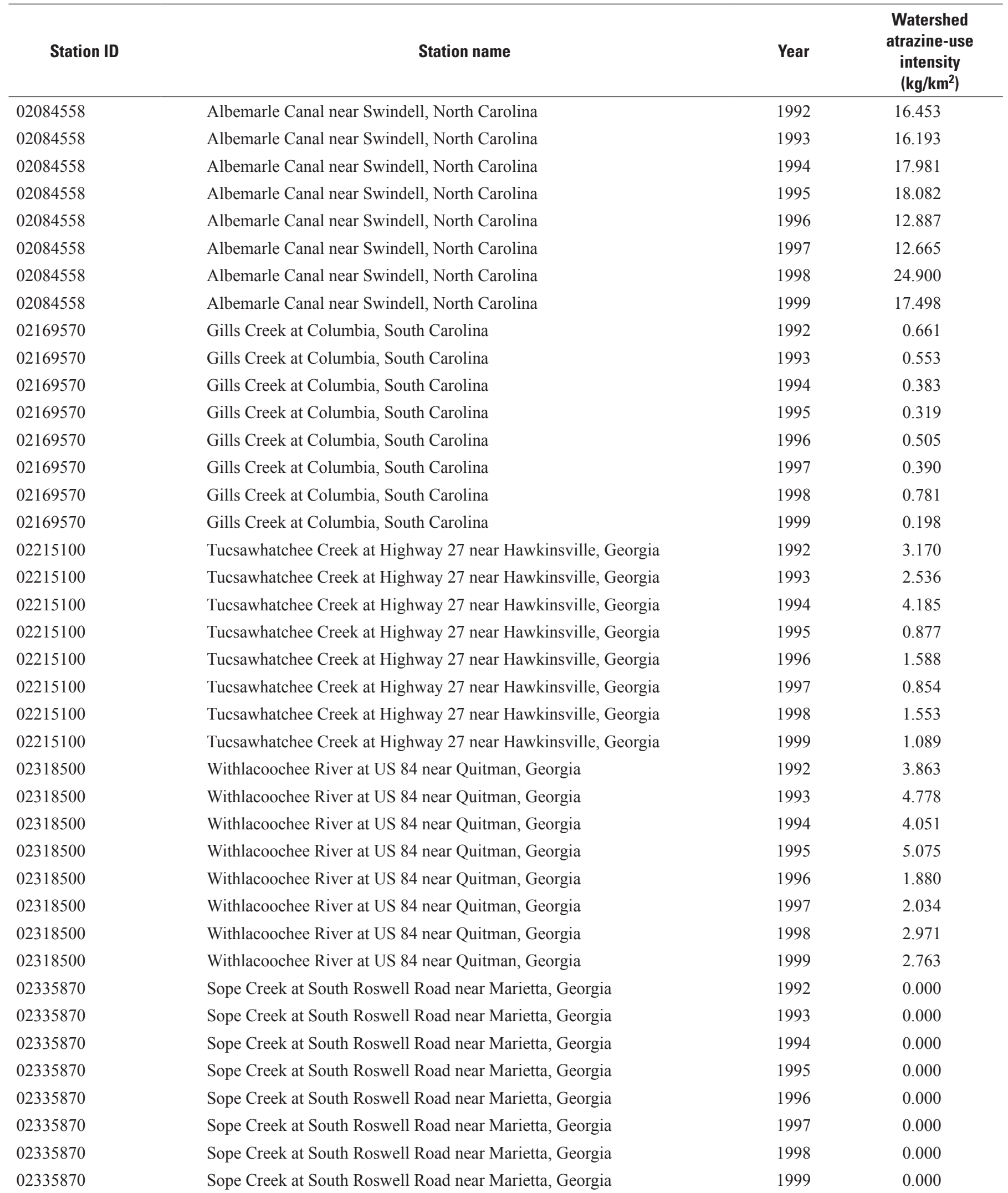


Appendix: Table A1. Annual atrazine-use intensity estimates for watersheds used to develop the Watershed Regressions for Pesticides model, 1992 through 1999. - Continued

$\left[\mathrm{kg} / \mathrm{km}^{2}\right.$, kilogram per square kilometer; —, no estimate]

\begin{tabular}{|c|c|c|c|}
\hline Station ID & Station name & Year & $\begin{array}{c}\text { Watershed } \\
\text { atrazine-use } \\
\text { intensity } \\
\left(\mathbf{k g} / \mathbf{k m}^{2}\right)\end{array}$ \\
\hline 02350080 & Lime Creek at County Road near Cobb, Georgia & 1993 & 3.790 \\
\hline 02350080 & Lime Creek at County Road near Cobb, Georgia & 1994 & 4.753 \\
\hline 02350080 & Lime Creek at County Road near Cobb, Georgia & 1996 & 5.455 \\
\hline 02350080 & Lime Creek at County Road near Cobb, Georgia & 1997 & 5.507 \\
\hline 02350080 & Lime Creek at County Road near Cobb, Georgia & 1998 & 9.929 \\
\hline 02350080 & Lime Creek at County Road near Cobb, Georgia & 1999 & 4.890 \\
\hline 02424000 & Cahaba River at Centreville, Alabama & 1992 & 0.133 \\
\hline 02424000 & Cahaba River at Centreville, Alabama & 1996 & 0.593 \\
\hline 02424000 & Cahaba River at Centreville, Alabama & 1997 & 0.037 \\
\hline 02424000 & Cahaba River at Centreville, Alabama & 1998 & 0.410 \\
\hline 02424000 & Cahaba River at Centreville, Alabama & 1999 & 0.146 \\
\hline 03167000 & Reed Creek at Grahams Forge, Virginia & 1992 & 3.851 \\
\hline 03167000 & Reed Creek at Grahams Forge, Virginia & 1993 & 4.562 \\
\hline 03167000 & Reed Creek at Grahams Forge, Virginia & 1994 & 7.883 \\
\hline 03167000 & Reed Creek at Grahams Forge, Virginia & 1995 & 2.671 \\
\hline 03176500 & New River at Glen Lyn, Virginia & 1994 & 3.663 \\
\hline 03176500 & New River at Glen Lyn, Virginia & 1995 & 1.333 \\
\hline 03176500 & New River at Glen Lyn, Virginia & 1996 & 4.010 \\
\hline 03176500 & New River at Glen Lyn, Virginia & 1997 & 0.407 \\
\hline 03176500 & New River at Glen Lyn, Virginia & 1998 & 3.157 \\
\hline 03176500 & New River at Glen Lyn, Virginia & 1999 & 1.805 \\
\hline 03216600 & Ohio River at Greenup Dam, Kentucky & 1992 & 3.926 \\
\hline 03216600 & Ohio River at Greenup Dam, Kentucky & 1993 & 2.784 \\
\hline 03216600 & Ohio River at Greenup Dam, Kentucky & 1994 & 3.400 \\
\hline 03216600 & Ohio River at Greenup Dam, Kentucky & 1995 & 2.923 \\
\hline 03216600 & Ohio River at Greenup Dam, Kentucky & 1996 & 3.102 \\
\hline 03216600 & Ohio River at Greenup Dam, Kentucky & 1997 & 3.831 \\
\hline 03216600 & Ohio River at Greenup Dam, Kentucky & 1998 & 4.108 \\
\hline 03216600 & Ohio River at Greenup Dam, Kentucky & 1999 & 3.084 \\
\hline
\end{tabular}


Appendix: Table A1. Annual atrazine-use intensity estimates for watersheds used to develop the Watershed Regressions for Pesticides model, 1992 through 1999._- Continued

$\left[\mathrm{kg} / \mathrm{km}^{2}\right.$, kilogram per square kilometer; —, no estimate $]$

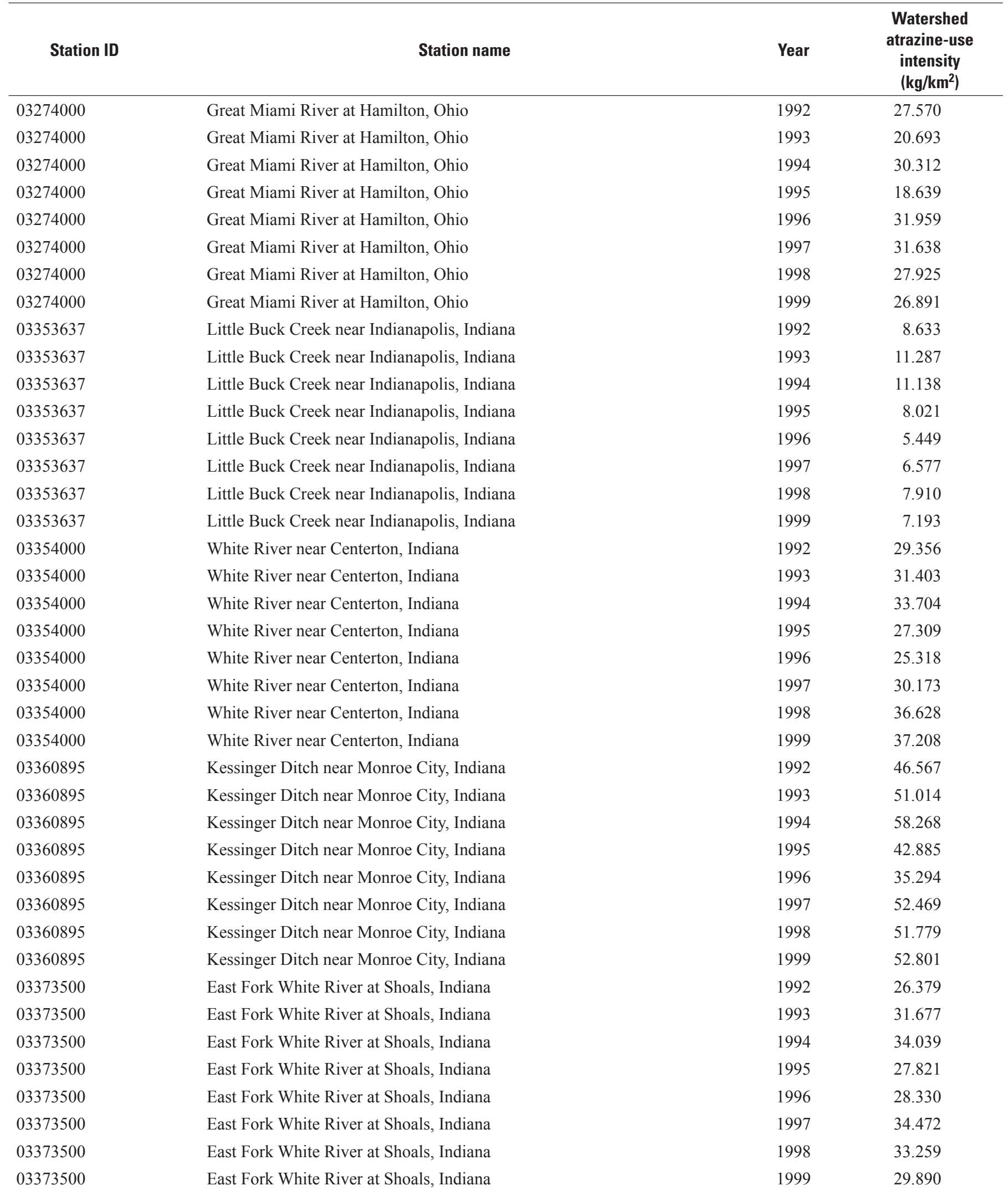


Appendix: Table A1. Annual atrazine-use intensity estimates for watersheds used to develop the Watershed Regressions for Pesticides model, 1992 through 1999. - Continued

$\left[\mathrm{kg} / \mathrm{km}^{2}\right.$, kilogram per square kilometer; —, no estimate]

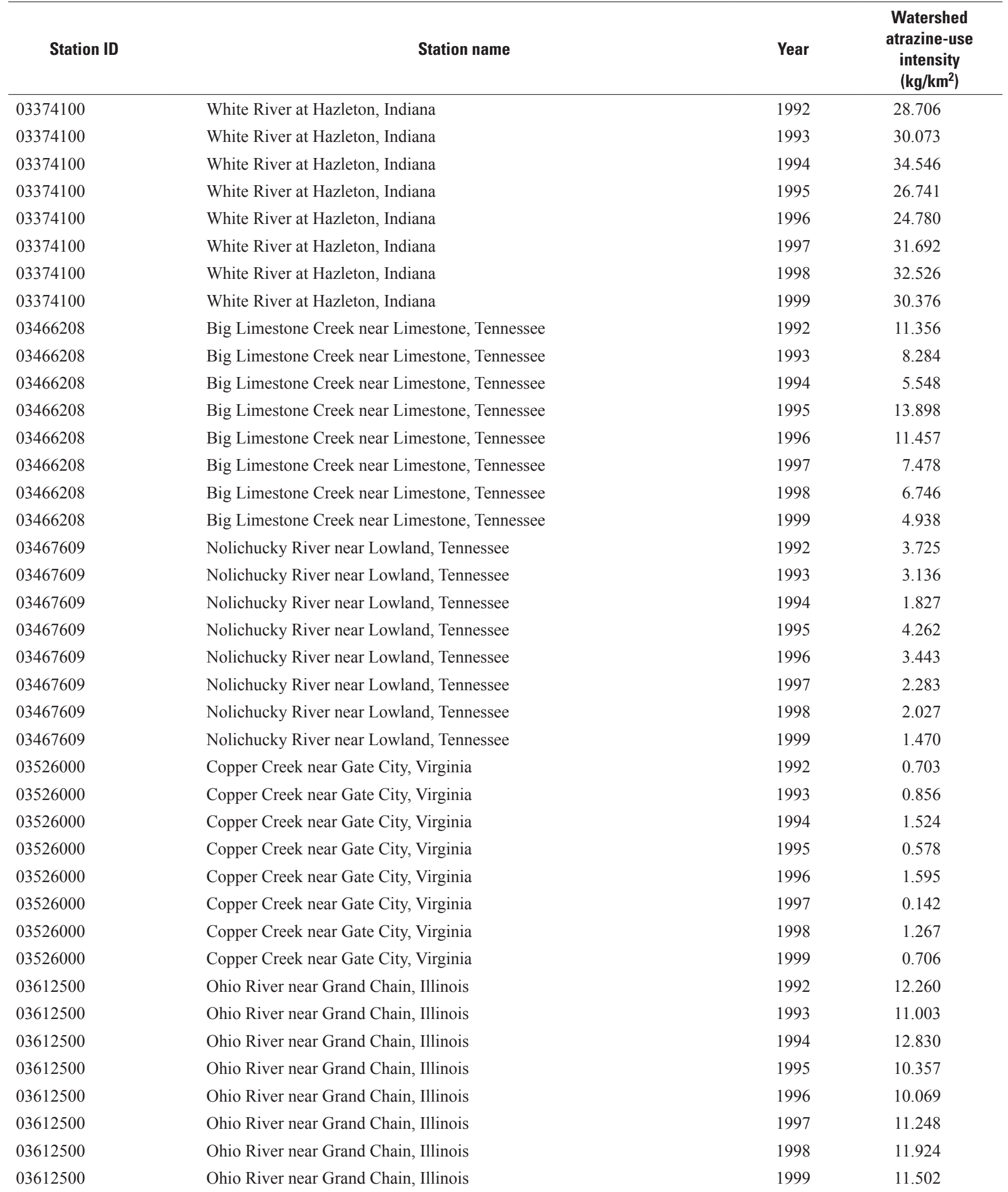


Appendix: Table A1. Annual atrazine-use intensity estimates for watersheds used to develop the Watershed Regressions for Pesticides model, 1992 through 1999._- Continued

$\left[\mathrm{kg} / \mathrm{km}^{2}\right.$, kilogram per square kilometer; - , no estimate $]$

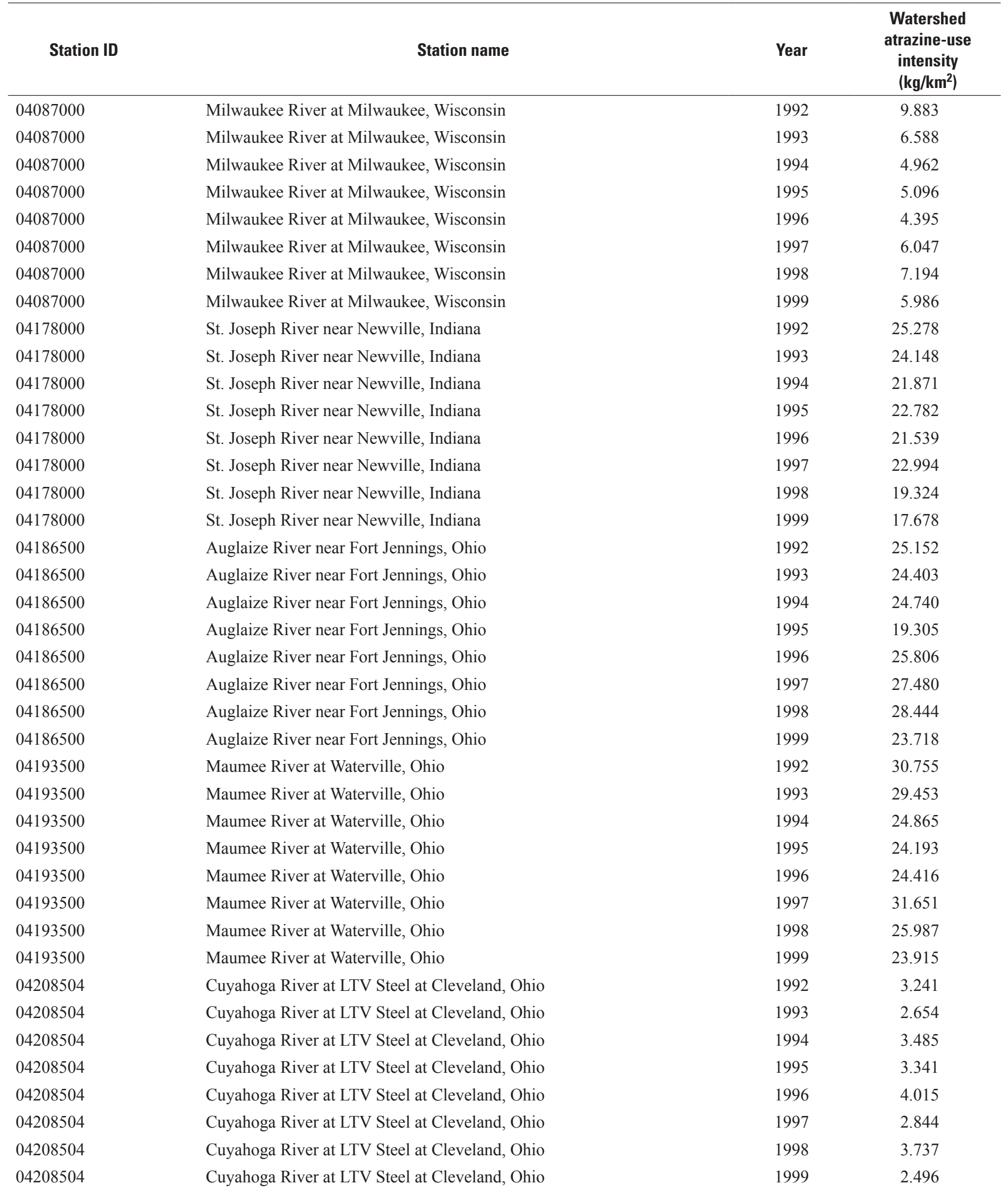


Appendix: Table A1. Annual atrazine-use intensity estimates for watersheds used to develop the Watershed Regressions for Pesticides model, 1992 through 1999. - Continued

$\left[\mathrm{kg} / \mathrm{km}^{2}\right.$, kilogram per square kilometer; —, no estimate]

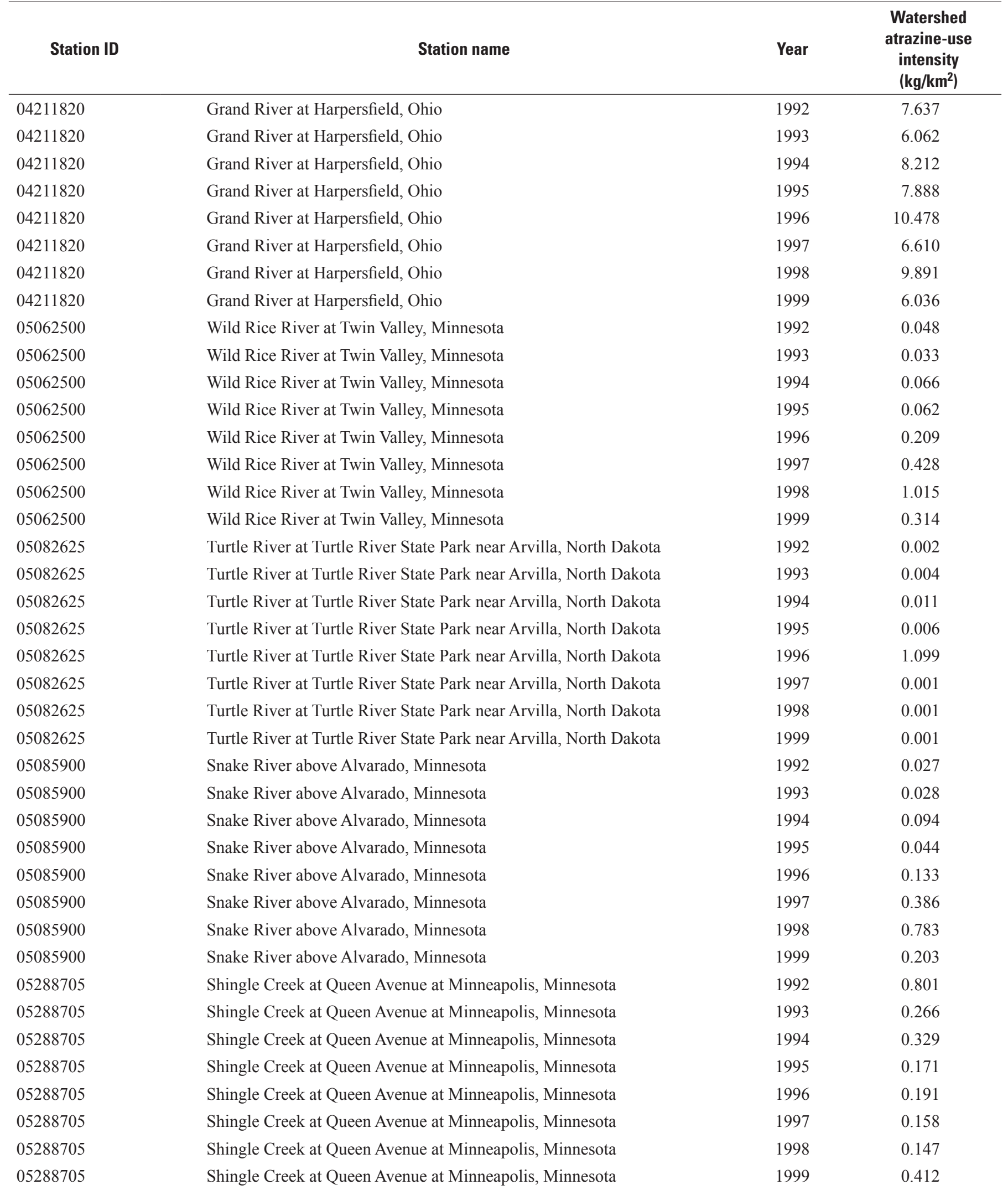


Appendix: Table A1. Annual atrazine-use intensity estimates for watersheds used to develop the Watershed Regressions for Pesticides model, 1992 through 1999._- Continued

$\left[\mathrm{kg} / \mathrm{km}^{2}\right.$, kilogram per square kilometer; —, no estimate $]$

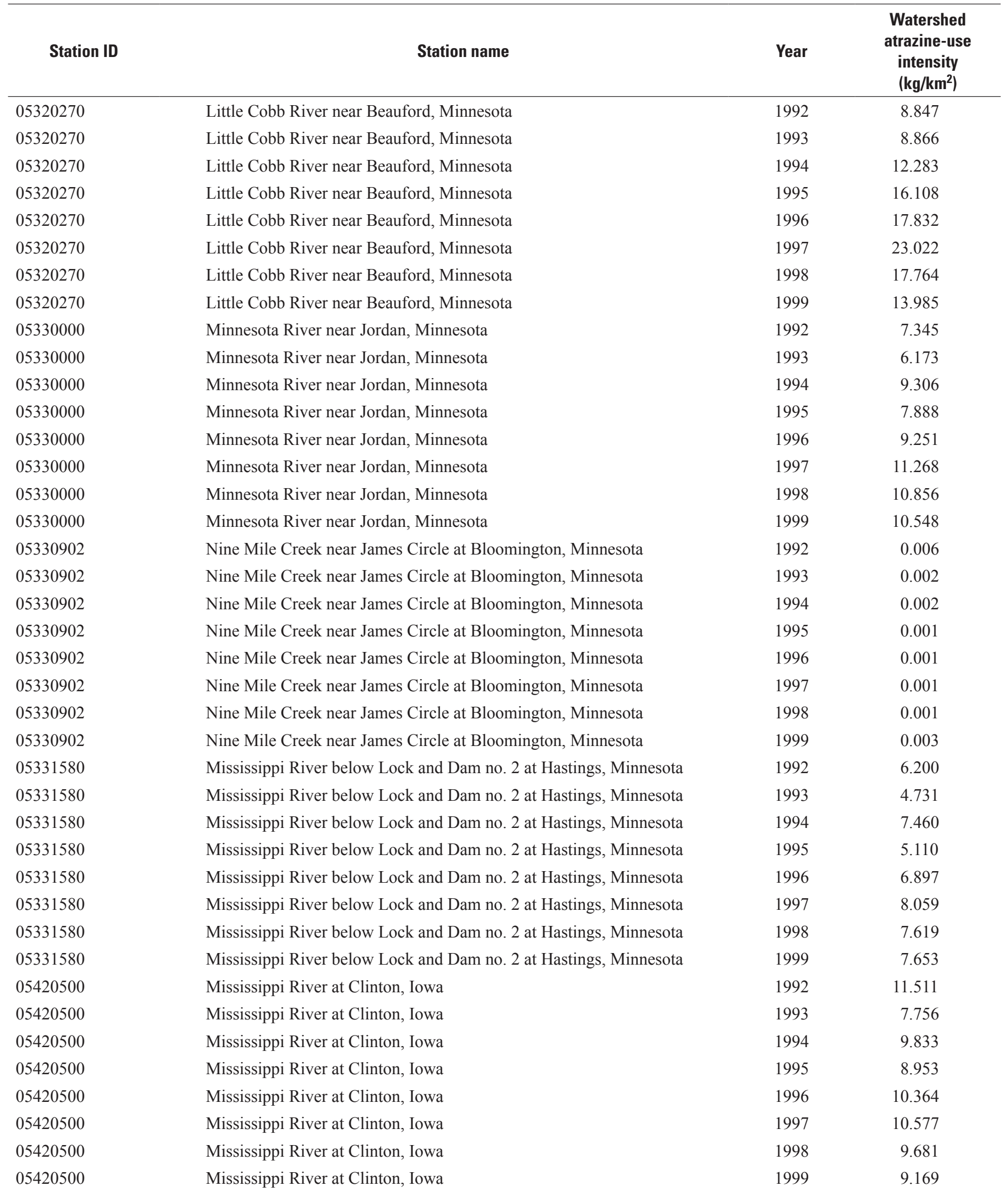


Appendix: Table A1. Annual atrazine-use intensity estimates for watersheds used to develop the Watershed Regressions for Pesticides model, 1992 through 1999. - Continued

$\left[\mathrm{kg} / \mathrm{km}^{2}\right.$, kilogram per square kilometer; —, no estimate]

\begin{tabular}{|c|c|c|c|}
\hline Station ID & Station name & Year & $\begin{array}{c}\text { Watershed } \\
\text { atrazine-use } \\
\text { intensity } \\
\left(\mathbf{k g} / \mathbf{k m}^{2}\right)\end{array}$ \\
\hline 05449500 & Iowa River near Rowan, Iowa & 1993 & 18.168 \\
\hline 05449500 & Iowa River near Rowan, Iowa & 1994 & 19.456 \\
\hline 05449500 & Iowa River near Rowan, Iowa & 1996 & 29.825 \\
\hline 05449500 & Iowa River near Rowan, Iowa & 1997 & 21.069 \\
\hline 05449500 & Iowa River near Rowan, Iowa & 1998 & 23.190 \\
\hline 05449500 & Iowa River near Rowan, Iowa & 1999 & 23.206 \\
\hline 05464220 & Wolf Creek near Dysart, Iowa & 1992 & 34.184 \\
\hline 05464220 & Wolf Creek near Dysart, Iowa & 1995 & 26.309 \\
\hline 05464220 & Wolf Creek near Dysart, Iowa & 1996 & 22.557 \\
\hline 05464220 & Wolf Creek near Dysart, Iowa & 1997 & 35.745 \\
\hline 05464220 & Wolf Creek near Dysart, Iowa & 1998 & 23.726 \\
\hline 05464220 & Wolf Creek near Dysart, Iowa & 1999 & 29.064 \\
\hline 05465500 & Iowa River at Wapello, Iowa & 1992 & 30.960 \\
\hline 05465500 & Iowa River at Wapello, Iowa & 1993 & 25.443 \\
\hline 05465500 & Iowa River at Wapello, Iowa & 1994 & 26.092 \\
\hline 05465500 & Iowa River at Wapello, Iowa & 1995 & 22.191 \\
\hline 05525500 & Sugar Creek at Milford, Illinois & 1994 & 48.298 \\
\hline 05525500 & Sugar Creek at Milford, Illinois & 1995 & 41.819 \\
\hline 05525500 & Sugar Creek at Milford, Illinois & 1996 & 35.595 \\
\hline 05525500 & Sugar Creek at Milford, Illinois & 1997 & 38.549 \\
\hline 05525500 & Sugar Creek at Milford, Illinois & 1998 & 49.676 \\
\hline 05525500 & Sugar Creek at Milford, Illinois & 1999 & 51.181 \\
\hline 05531500 & Salt Creek at Western Springs, Illinois & 1992 & 0.425 \\
\hline 05531500 & Salt Creek at Western Springs, Illinois & 1993 & 0.365 \\
\hline 05531500 & Salt Creek at Western Springs, Illinois & 1994 & 0.379 \\
\hline 05531500 & Salt Creek at Western Springs, Illinois & 1995 & 0.292 \\
\hline 05531500 & Salt Creek at Western Springs, Illinois & 1996 & 0.165 \\
\hline 05531500 & Salt Creek at Western Springs, Illinois & 1997 & 0.295 \\
\hline 05531500 & Salt Creek at Western Springs, Illinois & 1998 & 0.293 \\
\hline 05531500 & Salt Creek at Western Springs, Illinois & 1999 & 0.227 \\
\hline
\end{tabular}


Appendix: Table A1. Annual atrazine-use intensity estimates for watersheds used to develop the Watershed Regressions for Pesticides model, 1992 through 1999._- Continued

$\left[\mathrm{kg} / \mathrm{km}^{2}\right.$, kilogram per square kilometer; - , no estimate $]$

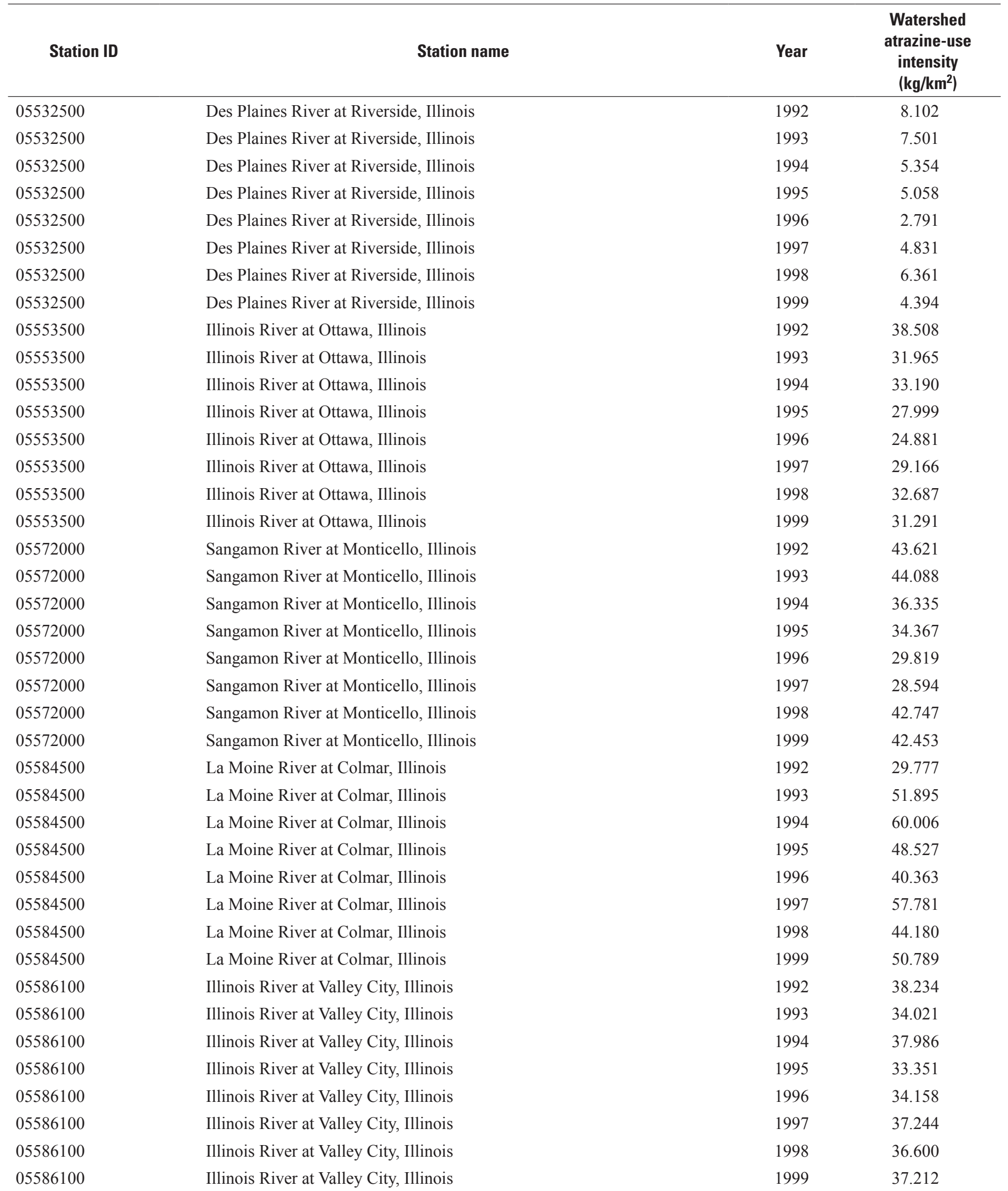


Appendix: Table A1. Annual atrazine-use intensity estimates for watersheds used to develop the Watershed Regressions for Pesticides model, 1992 through 1999. - Continued

$\left[\mathrm{kg} / \mathrm{km}^{2}\right.$, kilogram per square kilometer; - - no estimate]

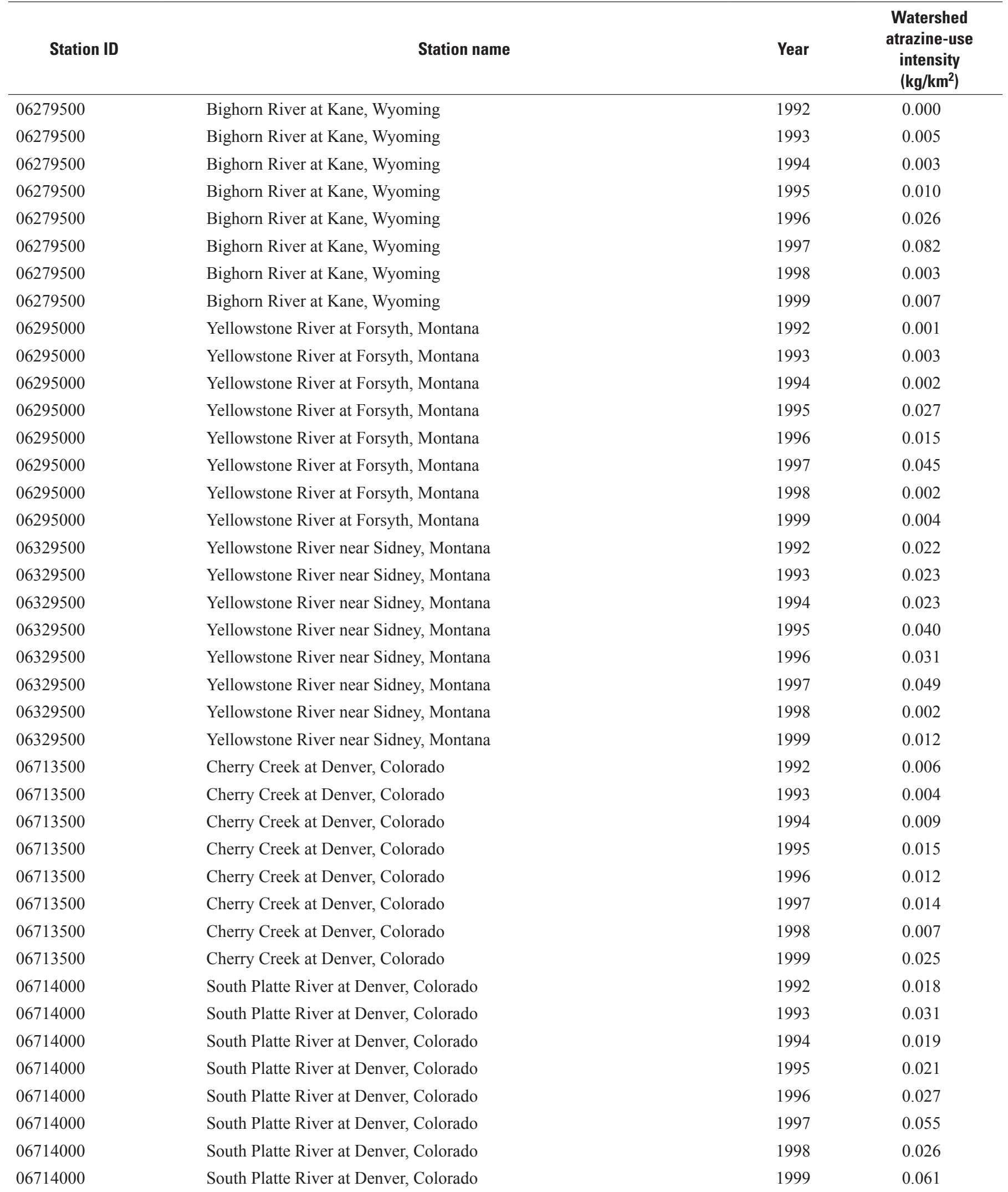


Appendix: Table A1. Annual atrazine-use intensity estimates for watersheds used to develop the Watershed Regressions for Pesticides model, 1992 through 1999._- Continued

$\left[\mathrm{kg} / \mathrm{km}^{2}\right.$, kilogram per square kilometer; - - , no estimate $]$

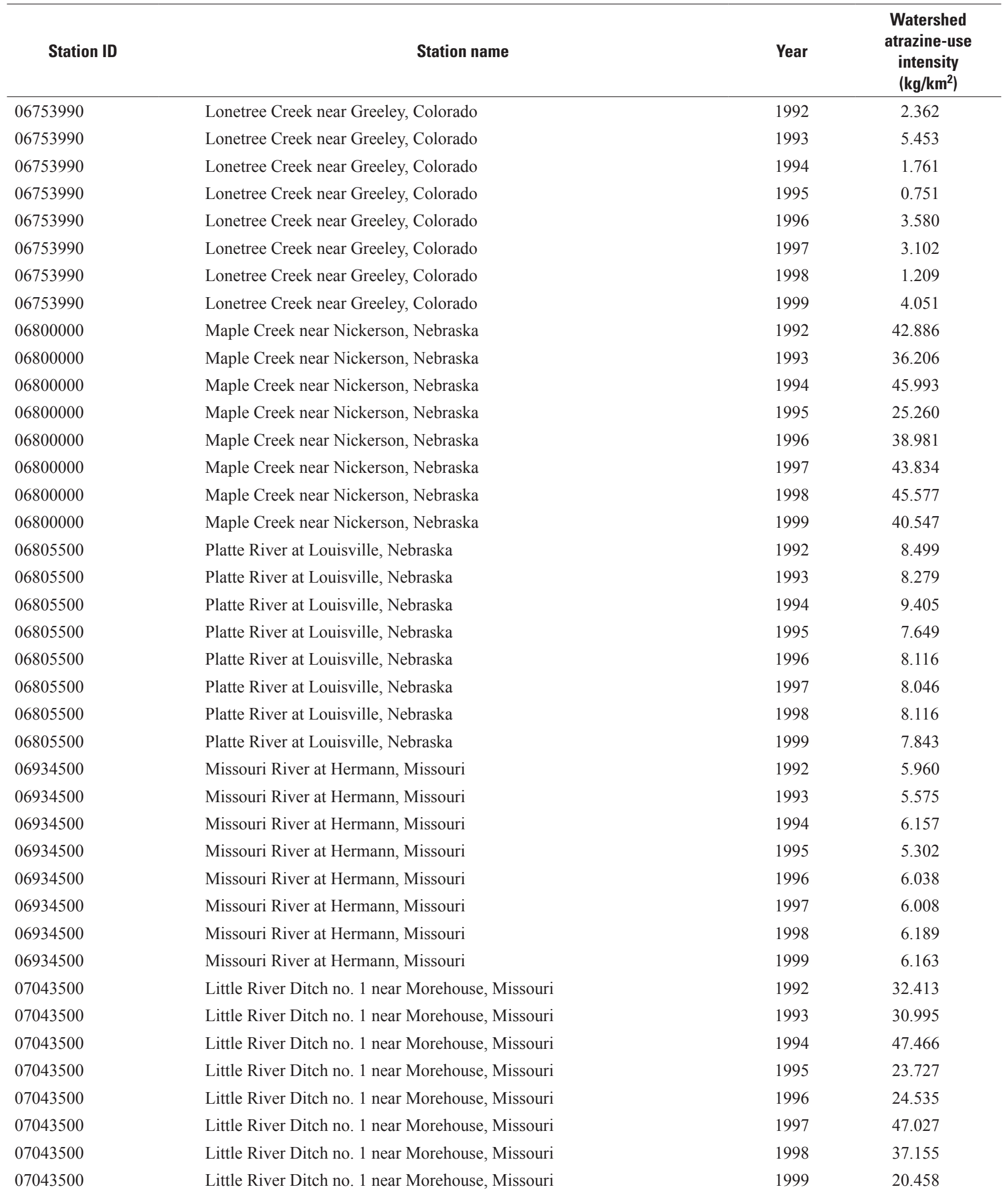


Appendix: Table A1. Annual atrazine-use intensity estimates for watersheds used to develop the Watershed Regressions for Pesticides model, 1992 through 1999. - Continued

$\left[\mathrm{kg} / \mathrm{km}^{2}\right.$, kilogram per square kilometer; - , no estimate $]$

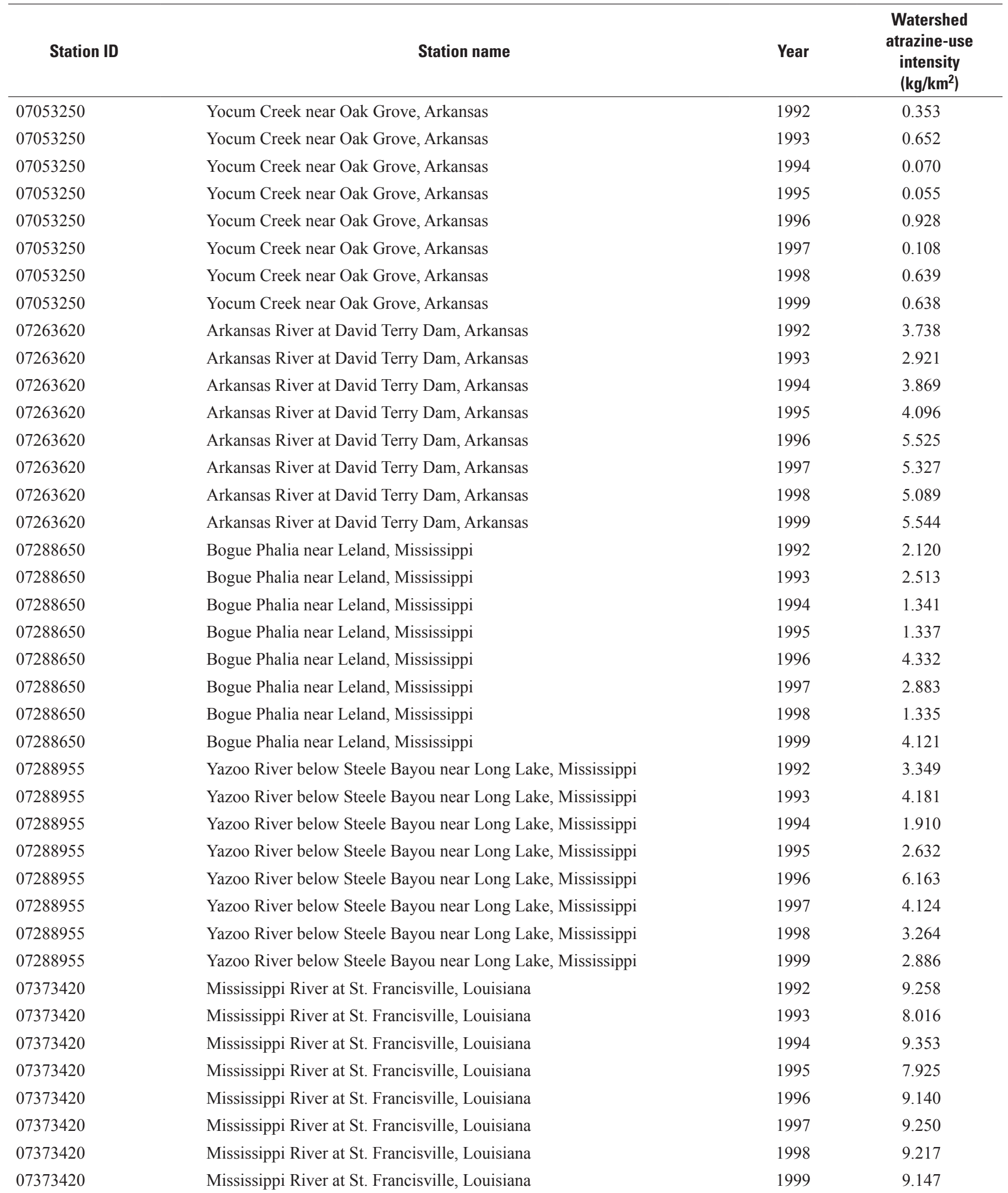


Appendix: Table A1. Annual atrazine-use intensity estimates for watersheds used to develop the Watershed Regressions for Pesticides model, 1992 through 1999._- Continued

$\left[\mathrm{kg} / \mathrm{km}^{2}\right.$, kilogram per square kilometer; - - , no estimate $]$

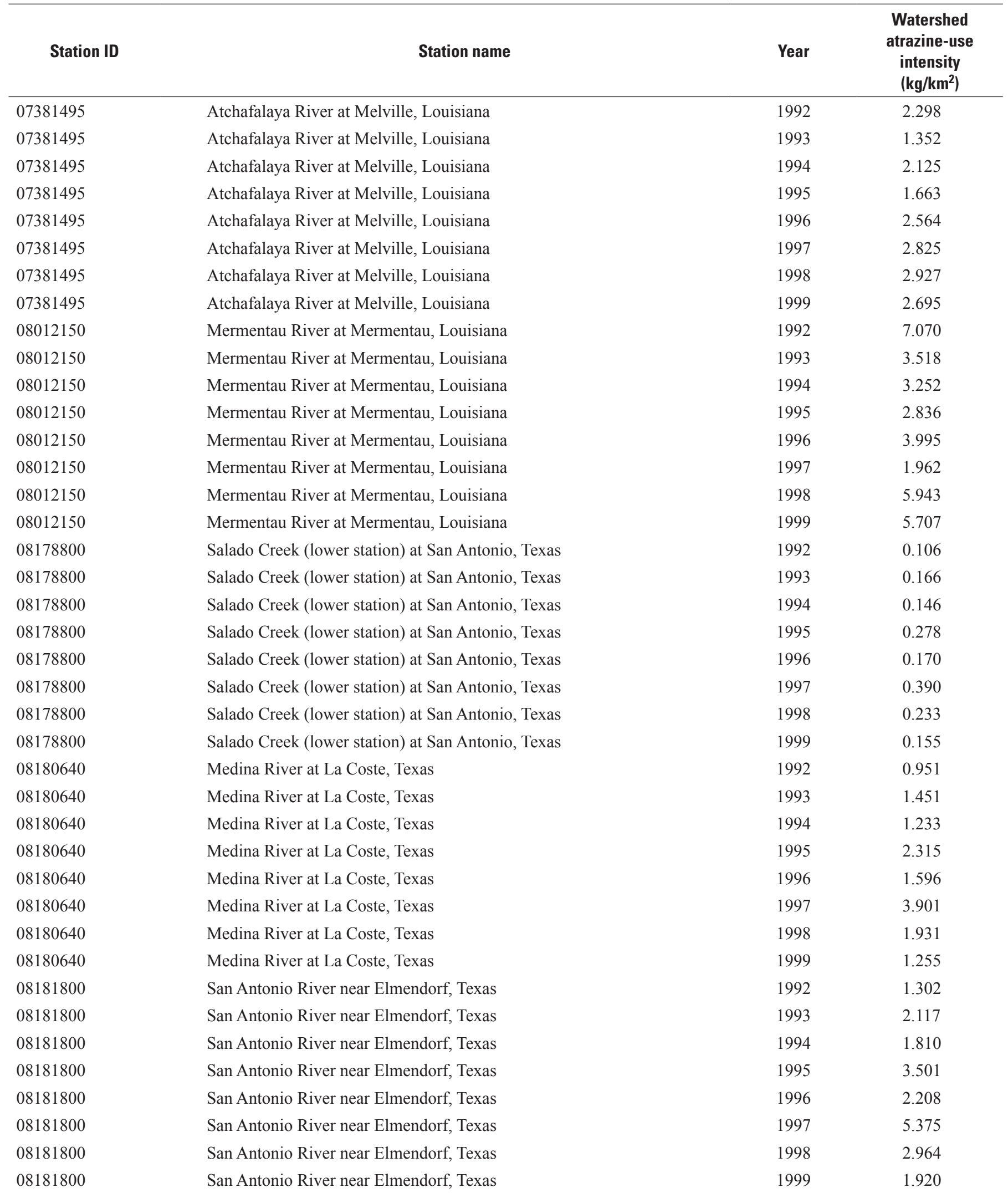


Appendix: Table A1. Annual atrazine-use intensity estimates for watersheds used to develop the Watershed Regressions for Pesticides model, 1992 through 1999. - Continued

$\left[\mathrm{kg} / \mathrm{km}^{2}\right.$, kilogram per square kilometer; - - no estimate]

\begin{tabular}{|c|c|c|c|}
\hline Station ID & Station name & Year & $\begin{array}{c}\text { Watershed } \\
\text { atrazine-use } \\
\text { intensity } \\
\left(\mathbf{k g} / \mathbf{k m}^{2}\right)\end{array}$ \\
\hline 09153290 & Reed Wash near Mack, Colorado & 1993 & 37.290 \\
\hline 09153290 & Reed Wash near Mack, Colorado & 1994 & 26.305 \\
\hline 09153290 & Reed Wash near Mack, Colorado & 1996 & 1.095 \\
\hline 09153290 & Reed Wash near Mack, Colorado & 1997 & 2.088 \\
\hline 09153290 & Reed Wash near Mack, Colorado & 1998 & 0.652 \\
\hline 09153290 & Reed Wash near Mack, Colorado & 1999 & 1.723 \\
\hline 09315000 & Green River at Green River, Utah & 1992 & 0.000 \\
\hline 09315000 & Green River at Green River, Utah & 1995 & 0.000 \\
\hline 09315000 & Green River at Green River, Utah & 1996 & 0.003 \\
\hline 09315000 & Green River at Green River, Utah & 1997 & 0.000 \\
\hline 09315000 & Green River at Green River, Utah & 1998 & 0.000 \\
\hline 09315000 & Green River at Green River, Utah & 1999 & 0.000 \\
\hline 09514000 & Buckeye Canal near Avondale, Arizona & 1992 & 0.004 \\
\hline 09514000 & Buckeye Canal near Avondale, Arizona & 1993 & 0.009 \\
\hline 09514000 & Buckeye Canal near Avondale, Arizona & 1994 & 0.067 \\
\hline 09514000 & Buckeye Canal near Avondale, Arizona & 1995 & 0.000 \\
\hline 09517000 & Hassayampa River near Arlington, Arizona & 1994 & 0.111 \\
\hline 09517000 & Hassayampa River near Arlington, Arizona & 1995 & 0.000 \\
\hline 09517000 & Hassayampa River near Arlington, Arizona & 1996 & 0.119 \\
\hline 09517000 & Hassayampa River near Arlington, Arizona & 1997 & 0.140 \\
\hline 09517000 & Hassayampa River near Arlington, Arizona & 1998 & 0.111 \\
\hline 09517000 & Hassayampa River near Arlington, Arizona & 1999 & 0.132 \\
\hline 10171000 & Jordan River at 1700 South Road at Salt Lake City, Utah & 1992 & 0.096 \\
\hline 10171000 & Jordan River at 1700 South Road at Salt Lake City, Utah & 1993 & 0.167 \\
\hline 10171000 & Jordan River at 1700 South Road at Salt Lake City, Utah & 1994 & 0.062 \\
\hline 10171000 & Jordan River at 1700 South Road at Salt Lake City, Utah & 1995 & 0.277 \\
\hline 10171000 & Jordan River at 1700 South Road at Salt Lake City, Utah & 1996 & 0.006 \\
\hline 10171000 & Jordan River at 1700 South Road at Salt Lake City, Utah & 1997 & 0.006 \\
\hline 10171000 & Jordan River at 1700 South Road at Salt Lake City, Utah & 1998 & 0.121 \\
\hline 10171000 & Jordan River at 1700 South Road at Salt Lake City, Utah & 1999 & 0.289 \\
\hline
\end{tabular}


Appendix: Table A1. Annual atrazine-use intensity estimates for watersheds used to develop the Watershed Regressions for Pesticides model, 1992 through 1999._- Continued

$\left[\mathrm{kg} / \mathrm{km}^{2}\right.$, kilogram per square kilometer; —, no estimate $]$

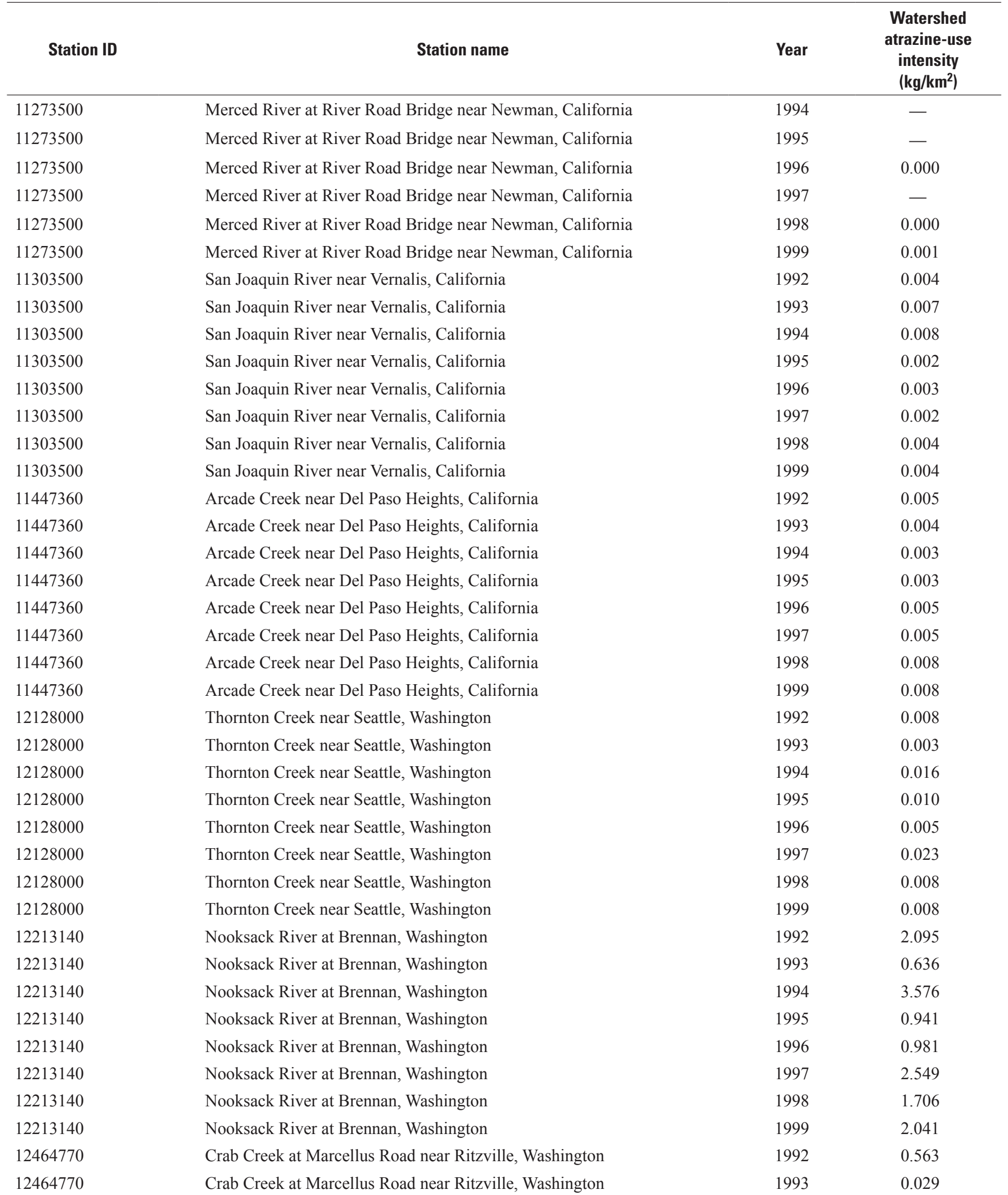


Appendix: Table A1. Annual atrazine-use intensity estimates for watersheds used to develop the Watershed Regressions for Pesticides model, 1992 through 1999. - Continued

$\left[\mathrm{kg} / \mathrm{km}^{2}\right.$, kilogram per square kilometer; - , no estimate $]$

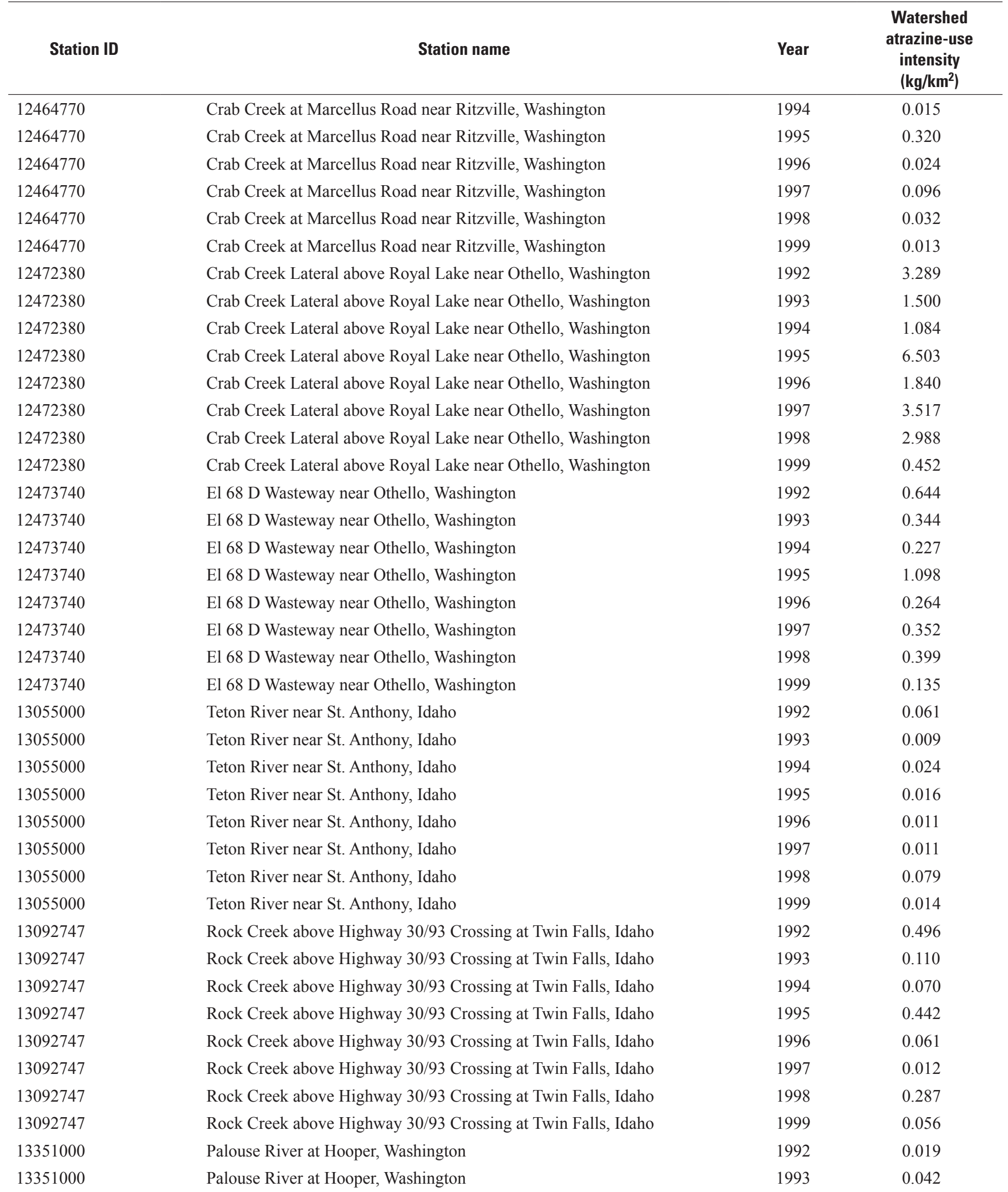


Appendix: Table A1. Annual atrazine-use intensity estimates for watersheds used to develop the Watershed Regressions for Pesticides model, 1992 through 1999._- Continued

$\left[\mathrm{kg} / \mathrm{km}^{2}\right.$, kilogram per square kilometer; —, no estimate $]$

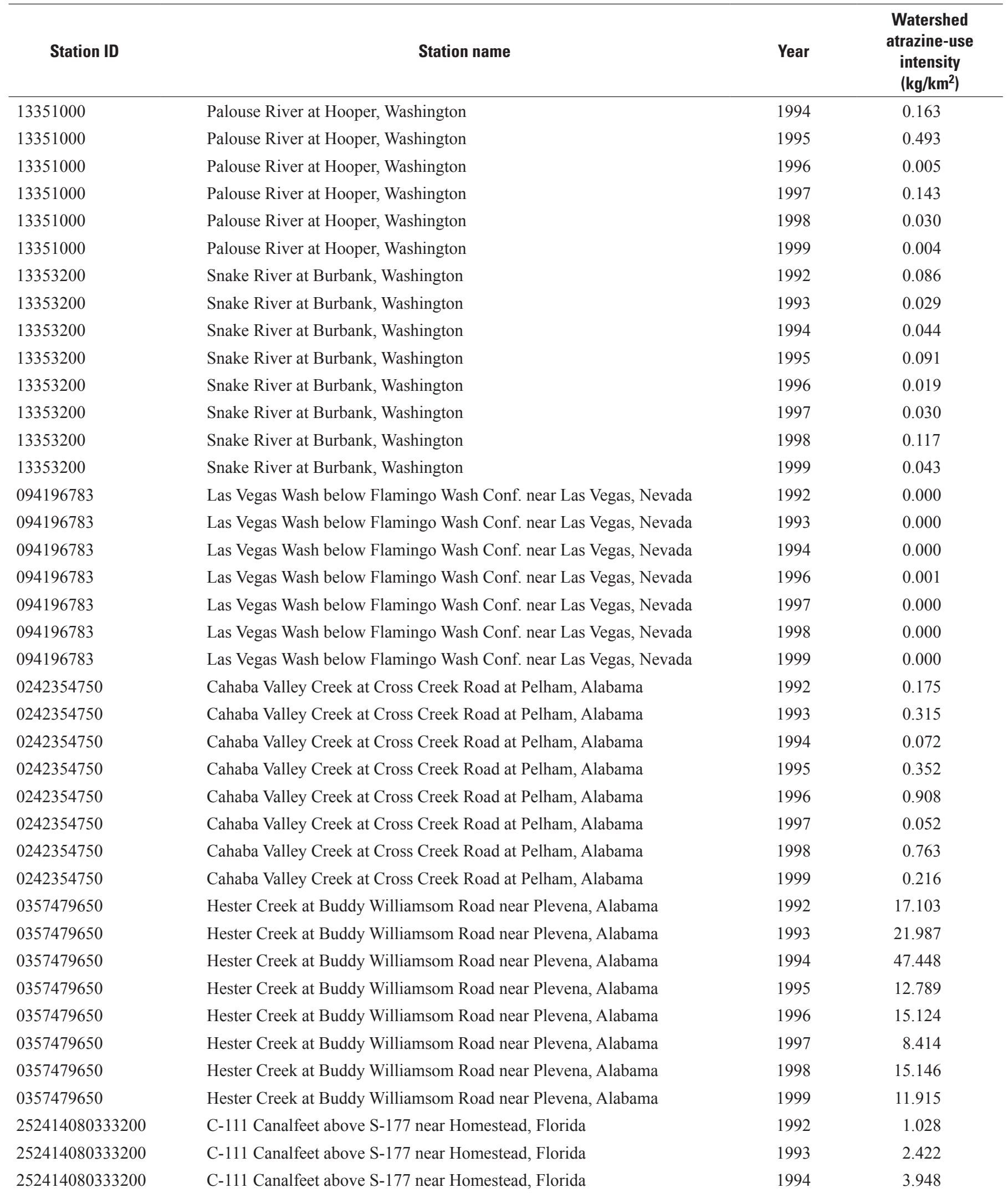


Appendix: Table A1. Annual atrazine-use intensity estimates for watersheds used to develop the Watershed Regressions for Pesticides model, 1992 through 1999. - Continued

$\left[\mathrm{kg} / \mathrm{km}^{2}\right.$, kilogram per square kilometer; - , no estimate $]$

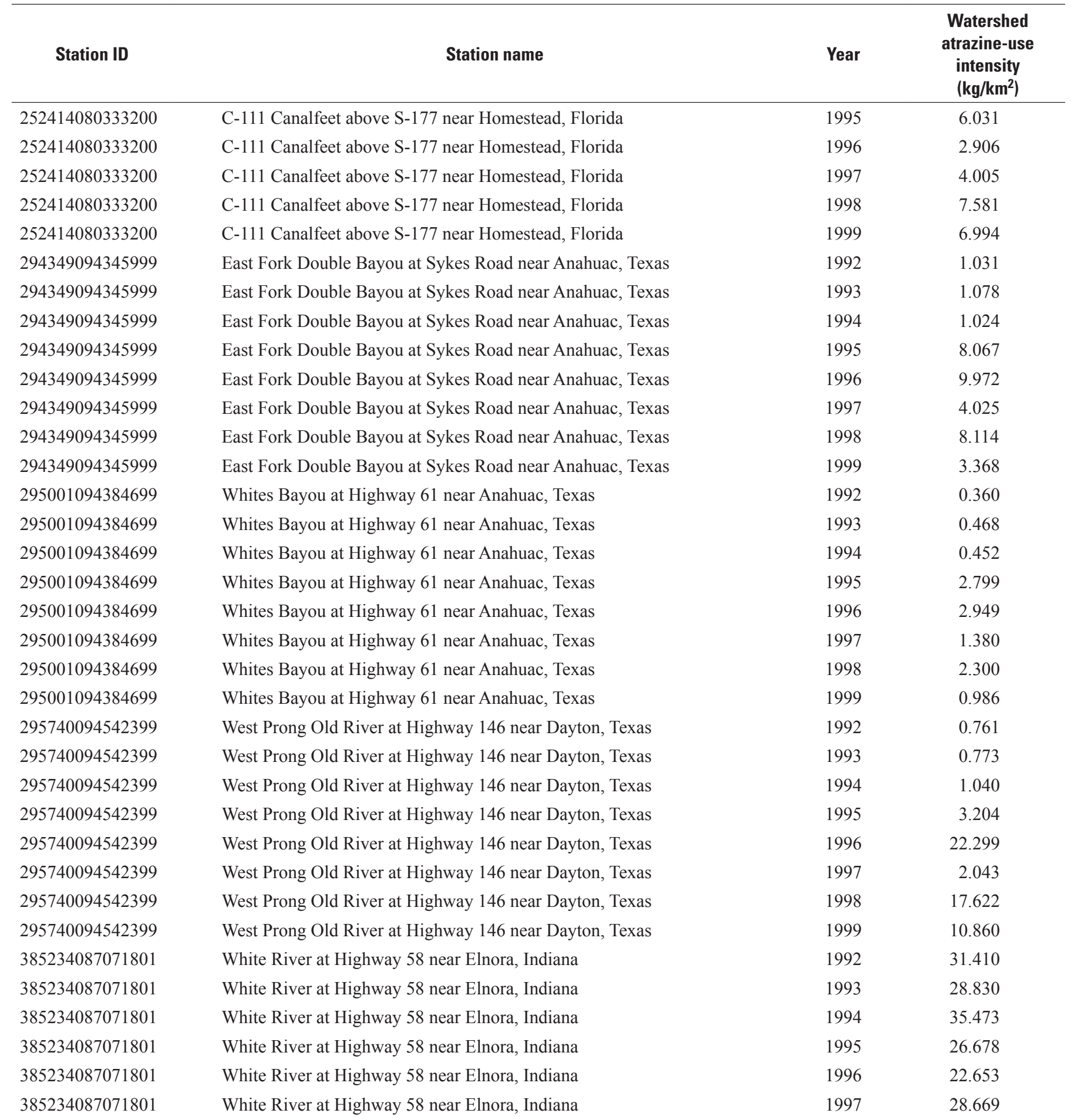


Appendix: Table A1. Annual atrazine-use intensity estimates for watersheds used to develop the Watershed Regressions for Pesticides model, 1992 through 1999. - Continued

$\left[\mathrm{kg} / \mathrm{km}^{2}\right.$, kilogram per square kilometer; - , no estimate $]$

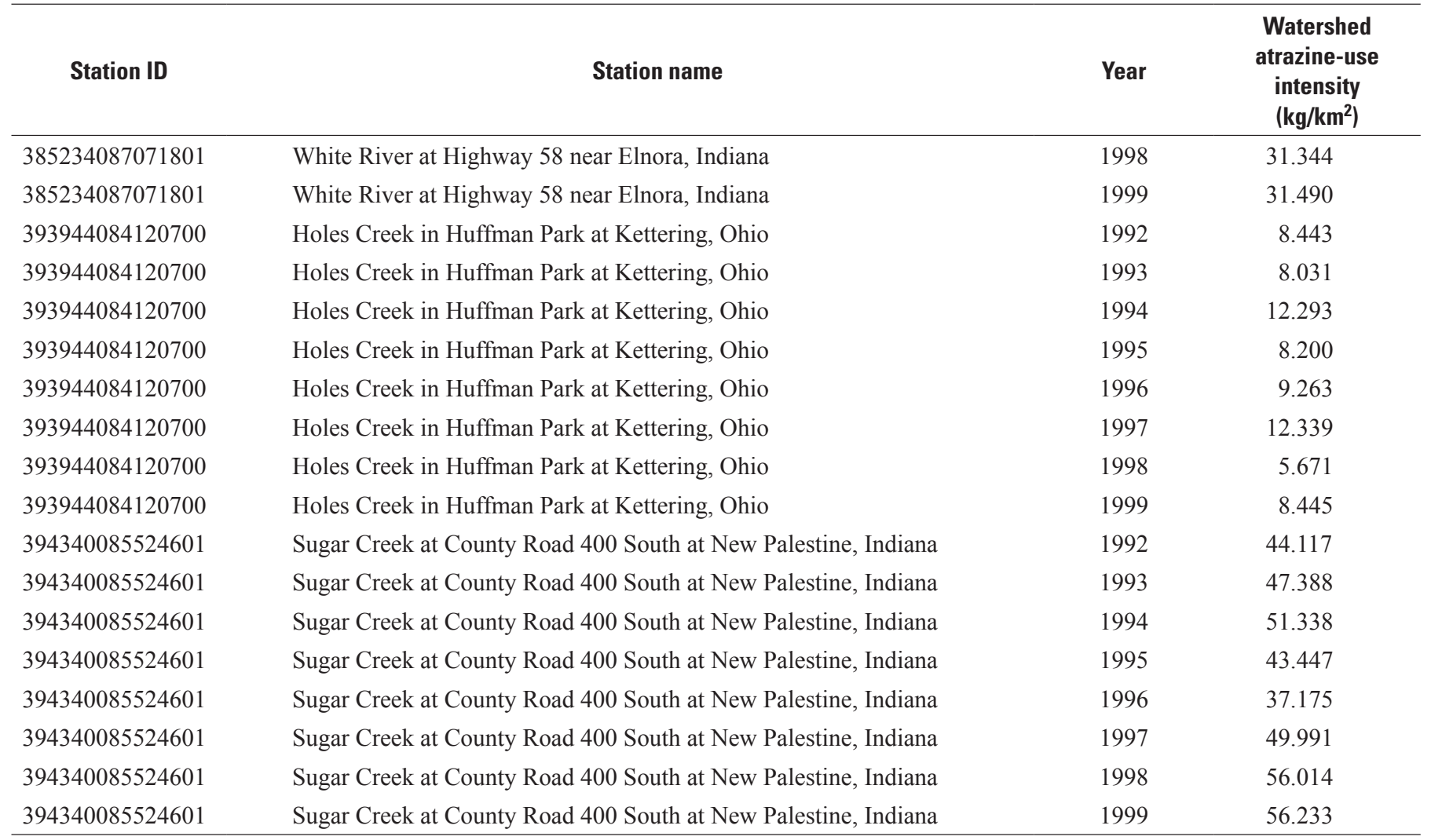




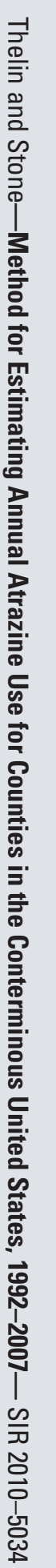

8 Printed on recycled paper 